\title{
Irradiation and PIE of Alloys X-750 and XM-19 (EPRI Phase III)
}

\author{
J.H. Jackson \\ M. Heighes \\ P.L. Andresen \\ July 2020
}

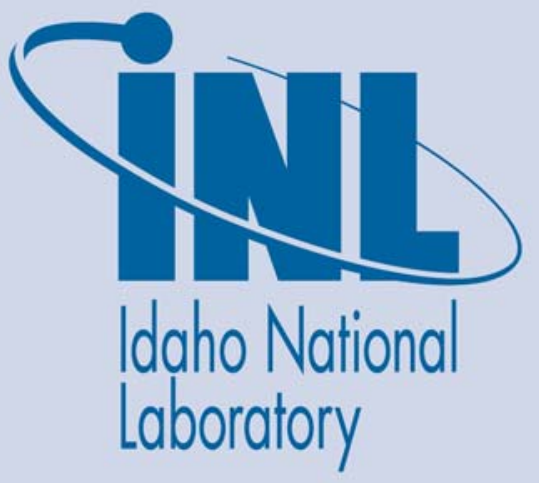

The INL is a U.S. Department of Energy National Laboratory operated by Battelle Energy Alliance 


\section{DISCLAIMER}

This information was prepared as an account of work sponsored by an agency of the U.S. Government. Neither the U.S. Government nor any agency thereof, nor any of their employees, makes any warranty, expressed or implied, or assumes any legal liability or responsibility for the accuracy, completeness, or usefulness, of any information, apparatus, product, or process disclosed, or represents that its use would not infringe privately owned rights. References herein to any specific commercial product, process, or service by trade name, trade mark, manufacturer, or otherwise, does not necessarily constitute or imply its endorsement, recommendation, or favoring by the U.S. Government or any agency thereof. The views and opinions of authors expressed herein do not necessarily state or reflect those of the U.S. Government or any agency thereof. 


\title{
Irradiation and PIE of Alloys X-750 and XM-19 (EPRI Phase III)
}

\author{
J.H. Jackson \\ M. Heighes \\ P.L. Andresen
}

July 2020

\begin{abstract}
Idaho National Laboratory Nuclear Science User Facilities Idaho Falls, Idaho 83415
\end{abstract}

http://www.inl.gov

Prepared for the U.S. Department of Energy Office of Nuclear Science Under DOE Idaho Operations Office Contract DE-AC07-05ID14517 


\title{
NSUF
}

\section{Irradiation and PIE of Alloys X-750 and XM-19 (EPRI Phase III)}

\author{
INL/EXT-20-58432
}

Revision 1

July 2020

\section{Approved by:}

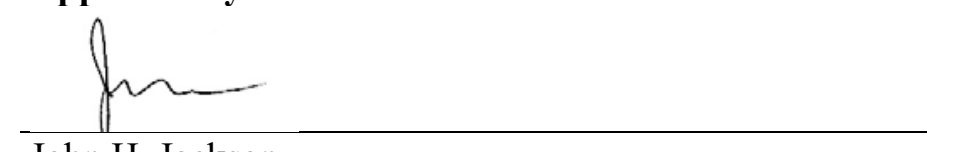

John H. Jackson

Date

Principal Investigator

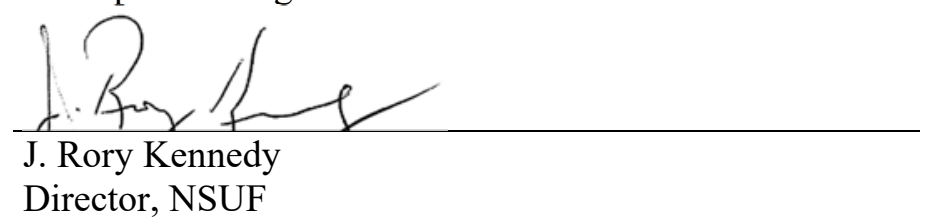

$05-15-2020$

$05-15-2020$

Date 


\section{SUMMARY}

The Nuclear Science User Facilities (NSUF) based at Idaho National Laboratory (INL), along with the Electric Power Research Institute (EPRI), formed an agreement to test representative alloys used as reactor structural materials as a pilot program to establish guidelines for future NSUF research programs. This report contains results from the portion of this program established as Phase III (of three phases), entailing irradiation and post-irradiation examination of select alloys typical of boiling water reactor (BWR) internal structural materials. Phases I and II are the subject of separate reports and represent baseline material test results and irradiation experiment design, respectively. The intent of this Phase III research program is to determine properties for the materials of interest after being irradiated at the Advanced Test Reactor (ATR) to three different target fast $(\mathrm{E}>1 \mathrm{MeV})$ fluences: $5.0 \times 10^{19} \mathrm{n} / \mathrm{cm}^{2}, 2.0 \mathrm{x}$ $10^{20} \mathrm{n} / \mathrm{cm}^{2}$, and $1.0 \times 10^{21} \mathrm{n} / \mathrm{cm}^{2}$. These correspond to irradiation damage levels (displacements per atom [dpa]) of approximately $0.08,0.30$, and $1.4 \mathrm{dpa}$, which represent comparable levels to (a) a previous study which looked at X-750 irradiated to $\sim 1 \times 10^{19} \mathrm{n} / \mathrm{cm}^{2}$, comparable to the lowest fluence; (b) approximately a medium level of fluence for BWR components; and (c) extended life ( 60 - 80 years) for BWR components. The materials chosen for this research are the nickel-based alloy X-750 and austenitic stainless steel XM-19. A spare core shroud upper support bracket of alloy X-750 was purchased by EPRI from Southern Co., and a section of XM-19 plate was purchased by EPRI from GE-Hitachi. These materials were sectioned at GE Global Research Center (GE-GRC), and parts were provided to INL for use in this pilot project.

Following completion of the irradiations at ATR in the water-cooled center flux trap, irradiation assisted stress corrosion cracking (IASCC), fracture toughness (FT), tensile testing, and transmission electron microscopy (TEM) studies were conducted at INL. Testing under normal water chemistry (NWC) conditions indicated a negligible effect on crack growth rate (CGR) at the medium and high fluence irradiation levels compared to the CGRs measured in unirradiated material. Under hydrogen water chemistry (HWC) conditions, only a modest increase in CGR was measured at the high fluence level compared to the medium fluence level. Tensile and FT measurements were far more sensitive to irradiation level, especially in the case of alloy X-750, with an increase of 21,29 , and $57 \%$ of yield strength compared to unirradiated levels for the low, medium, and high fluences, respectively, and an approximate 4,18 , and $41 \%$ reduction in FT for the low, medium, and high fluences, respectively. Additionally, TEM analyses showed an increase in dislocation loop size as a function of irradiation dose for both alloys, but a negligible change in loop density. 


\section{CONTENTS}

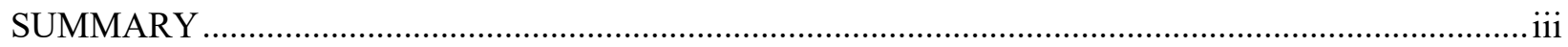

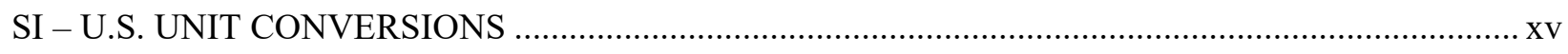

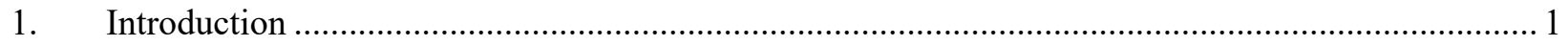

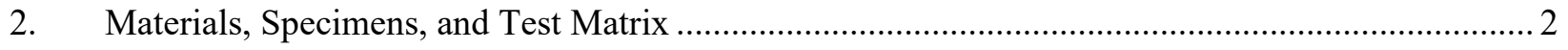

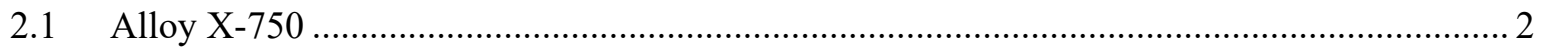

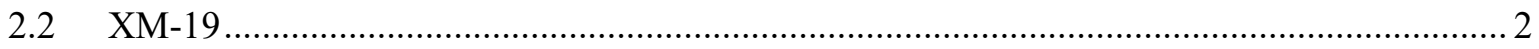

2.3 Specimens for Mechanical Properties Testing ............................................................... 2

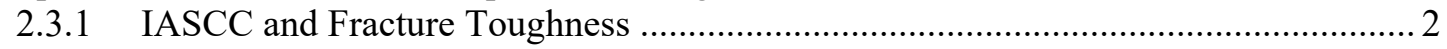

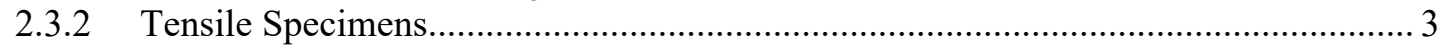

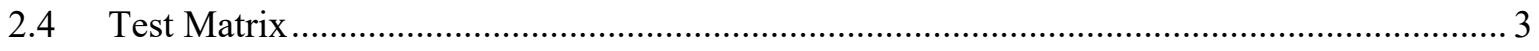

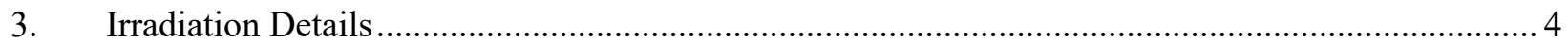

3.1 Irradiation Experiment Capsules and Specimen Layout .................................................... 5

3.1.1 Flow Restriction Issue and Mitigation ................................................................ 7

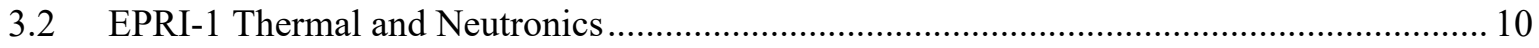

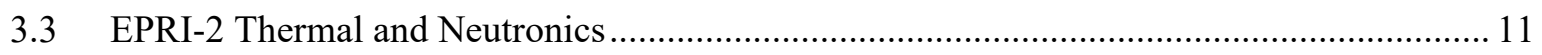

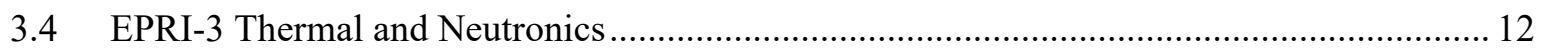

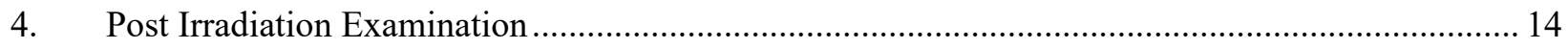

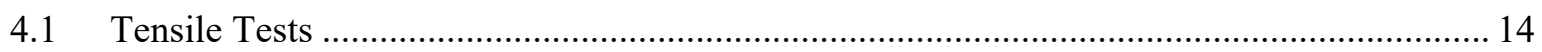

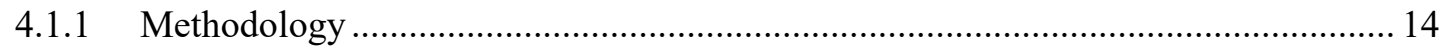

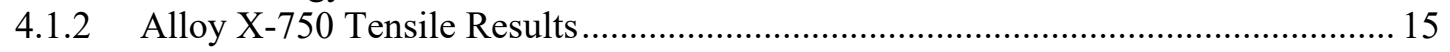

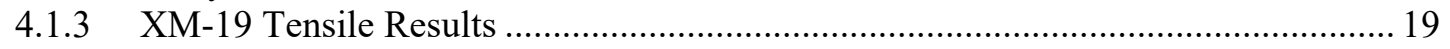

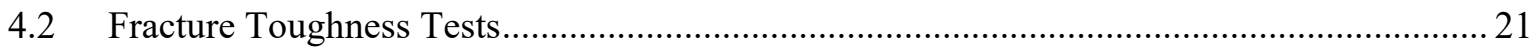

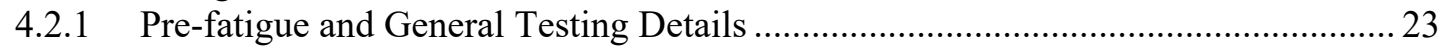

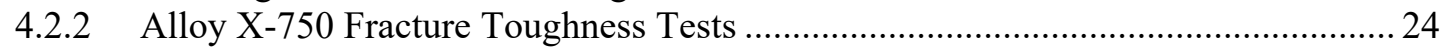

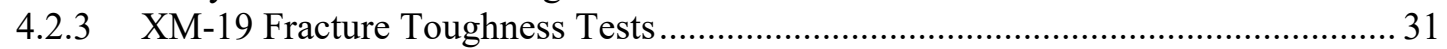

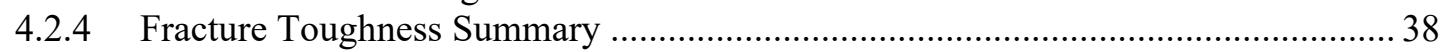

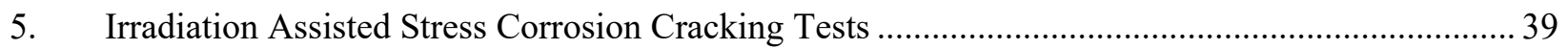

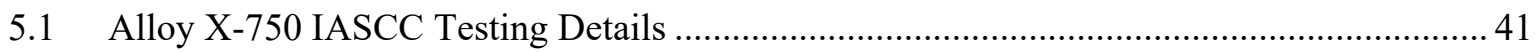

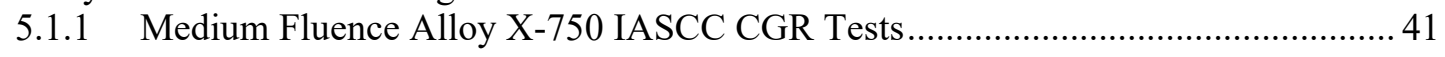

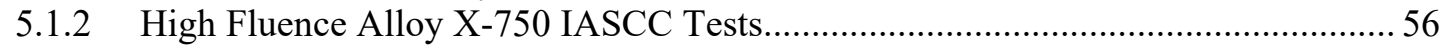

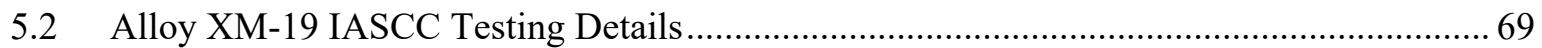

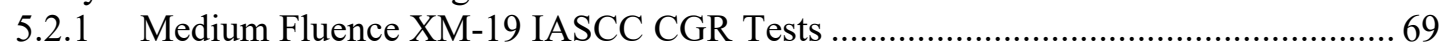

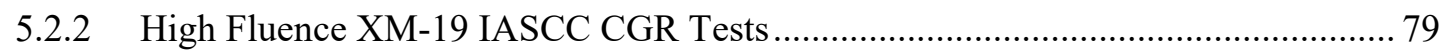

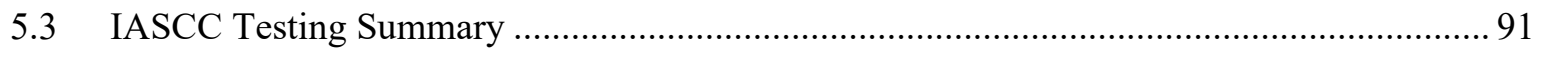

6. Transmission Electron Microscopy …............................................................................... 102

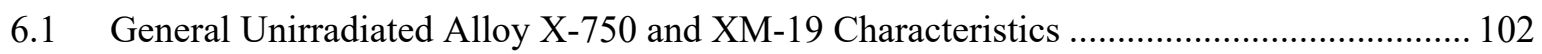

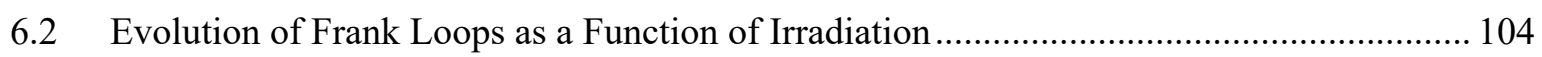

6.3 Stability of $\gamma^{\prime}$ Precipitates in Irradiated Alloy X-750 .................................................... 106 
7. Discussion and Conclusions

8. References

\section{FIGURES}

Figure 2-1: 0.4T-CT specimen for IASCC and fracture toughness (dimensions in inches)...................... 3

Figure 2-2: Standard tensile specimen (dimensions in inches)......................................................... 3

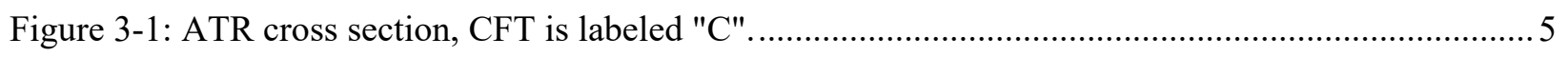

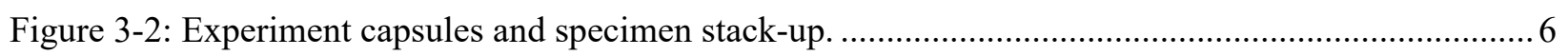

Figure 3-3: Generalized ATR axial flux profile (dimensions in inches) ................................................... 7

Figure 3-4: Photograph showing buildup of crud in specimen package flow channel. .............................. 8

Figure 3-5: Cruciform offset spacer to mitigate flow restriction issue. .................................................. 8

Figure 3-6: Additional flow restriction mitigation employed for EPRI-3-2 . ......................................... 9

Figure 3-7: Centering spacers designed to prevent twisting of specimen packages. .............................. 10

Figure 4-1: Typical tensile testing setup after installing a three-zone furnace and integration of an

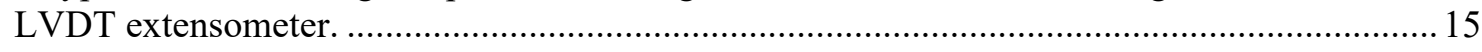

Figure 4-2: Schematic and photograph of LVDT strain measurement system. ...................................... 15

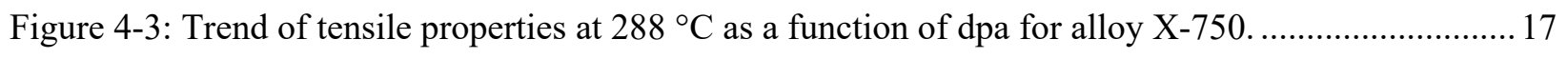

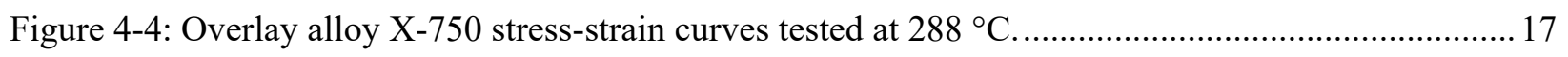

Figure 4-5: Vickers hardness measurements across the diameter of medium fluence alloy X-750

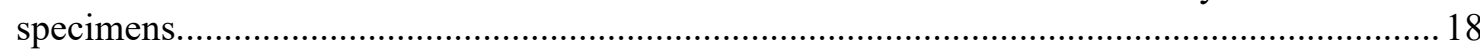

Figure 4-6: Micrographs showing consistent microstructure of medium fluence alloy X-750

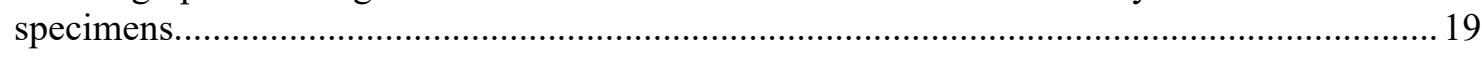

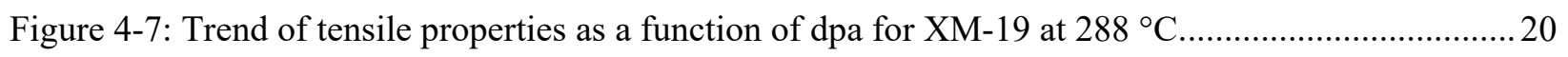

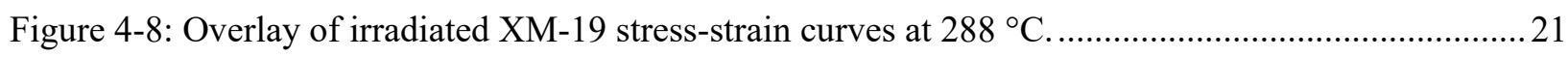

Figure 4-9: DCPD current inputs and potential leads. ...................................................................... 22

Figure 4-10: Exploded view of clevis-in-clevis specimen loading assembly showing zirconia

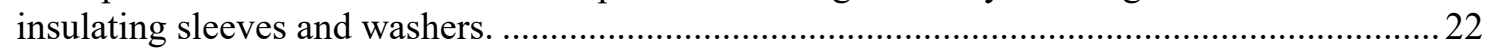

Figure 4-11: J-R curve and fracture surface for 10A0002A02 (alloy X-750, $0.086 \mathrm{dpa}$ ) specimen..........25

Figure 4-12: J-R curve and fracture surface for 10A0002A07 (alloy X-750, $0.086 \mathrm{dpa}$ ) specimen..........26

Figure 4-13: J-R curve and fracture surface for 10A0002A09 (alloy X-750, $0.306 \mathrm{dpa}$ ) specimen..........27

Figure 4-14: J-R curve and fracture surface for 10A0002A11 (alloy X-750, $0.307 \mathrm{dpa}$ ) specimen..........27

Figure 4-15: Force, displacement, crack growth, and fracture surface for 10A0002B08 (alloy X$750,1.54 \mathrm{dpa}$ ) specimen. 28 
Figure 4-16: Force, displacement, crack growth, and fracture surface for 10A0002B10 (alloy X$750,1.54 \mathrm{dpa})$ specimen.

Figure 4-17: Force vs. displacement and fracture surface for 10A0002C03 (alloy X-750, 1.49 dpa) specimen. 29

Figure 4-18: Closeup of fracture surface for 10A0002C03 (alloy X-750, $1.49 \mathrm{dpa}$ ). 30

Figure 4-19: Overlay plot of alloy X-750 J-R curves for baseline, low, and medium fluence tests at $288^{\circ} \mathrm{C}$.

Figure 4-20: J-R curve and fracture surface for 10A0001A02 (XM-19, $0.08 \mathrm{dpa}$ ) specimen. .32

Figure 4-21: J-R curve and fracture surface for 10A0001A07 (XM-19, $0.08 \mathrm{dpa}$ ) specimen.................... 33

Figure 4-22: J-R curve and fracture surface for 10A0001B01 (XM-19, $0.288 \mathrm{dpa}$ ) specimen. ................. 34

Figure 4-23: J-R curve and fracture surface for 10A0001B02 (XM-19, $0.291 \mathrm{dpa}$ ) specimen. ................. 34

Figure 4-24: J-R curve and fracture surface for 10A0001D01 (XM-19, $1.43 \mathrm{dpa}$ ) specimen...................35

Figure 4-25: J-R curve and fracture surface for 10A0001D05 (XM-19, $1.47 \mathrm{dpa}$ ) specimen................... 36

Figure 4-26: J-R curve and fracture surface for 10A0001E04 (XM-19, $1.41 \mathrm{dpa})$ specimen..................... 36

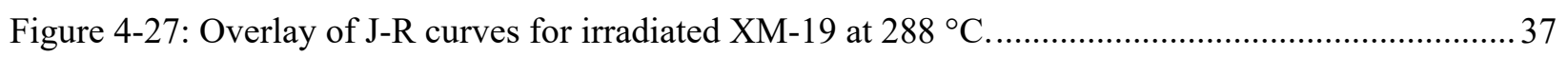

Figure 5-1: IASCC CGR overview of specimen 10A0002A08 of alloy X-750 irradiated to 0.30

dpa.

Figure 5-2: In-situ fatigue pre-cracking of specimen 10A0002A08 of alloy X-750 irradiated to

$0.30 \mathrm{dpa}$.

Figure 5-3: Initial IASCC response of specimen 10A0002A08 of alloy X-750 irradiated to 0.30 dpa.

Figure 5-4: IASCC response overview of transition to HWC and return to NWC under constant K for specimen 10A0002A08 of alloy X-750 irradiated to $0.30 \mathrm{dpa}$.

Figure 5-5: IASCC response overview of gentle cycling followed by return to constant $\mathrm{K}$ for specimen 10A0002A08 of alloy X-750 irradiated to $0.30 \mathrm{dpa}$.

Figure 5-6: Enlarged detail view of Figure 5-5 transition to higher constant $\mathrm{K}$ and second transition to HWC for specimen 10A0002A08 of alloy X-750 irradiated to $0.30 \mathrm{dpa}$.

Figure 5-7: IASCC response during and after crack front straightening and at higher $\mathrm{K}$ under HWC and NWC for specimen 10A0002A08 of alloy X-750 irradiated to $0.30 \mathrm{dpa}$.

Figure 5-8: Detail view of crack front straightening portion of Figure 5-7 for specimen 10A0002A08 of alloy X-750 irradiated to $0.30 \mathrm{dpa}$.

Figure 5-9: Final IASCC test steps for specimen 10A0002A08 of alloy X-750 irradiated to 0.30 dpa.

Figure 5-10: SEM macrograph showing fracture surface of specimen 10A0002A08 of alloy X750 irradiated to $0.30 \mathrm{dpa}$.

Figure 5-11: IASCC CGR test overview for specimen 10A0002A10 of alloy X-750 irradiated to $0.31 \mathrm{dpa}$. 48

Figure 5-12: In-situ fatigue pre-cracking of specimen 10A0002A10 of alloy X-750 irradiated to $0.31 \mathrm{dpa}$. 49 
Figure 5-13: Transition to intergranular fracture and initial CGR under NWC for specimen 10A0002A 10 of alloy X-750 irradiated to $0.31 \mathrm{dpa}$.

Figure 5-14: IASCC response for initial transition to HWC for specimen 10A0002A10 of alloy $\mathrm{X}-750$ irradiated to $0.31 \mathrm{dpa}$.

Figure 5-15: Effect of falling K (-dK/da) on NWC IASCC CGR for specimen 10A0002A10 of alloy X-750 irradiated to 0.31 dpa.

Figure 5-16: IASCC CGR under lower applied K and HWC for specimen 10A0002A10 of alloy $\mathrm{X}-750$ irradiated to $0.31 \mathrm{dpa}$. .52

Figure 5-17: Effect of rising $\mathrm{K}(+\mathrm{dK} / \mathrm{da})$ on NWC IASCC CGR for specimen 10A0002A10 of alloy X-750 irradiated to $0.31 \mathrm{dpa}$.

Figure 5-18: IASCC CGR following increased applied K and in HWC for specimen 10A0002A10 of alloy X-750 irradiated to $0.31 \mathrm{dpa}$

Figure 5-19: IASCC CGR response under gentle cycling to straighten the crack front for specimen 10A0002A10 of alloy X-750 irradiated to $0.31 \mathrm{dpa}$.

Figure 5-20: IASCC CGR response as $\mathrm{K}$ is further increased for specimen 10A0002A10 of alloy

$\mathrm{X}-750$ irradiated to $0.31 \mathrm{dpa}$.

Figure 5-21: Final steps of IASCC test of specimen 10A0002A10 of alloy X-750 irradiated to $0.31 \mathrm{dpa}$. .55

Figure 5-22: SEM micrograph showing the fracture surface of specimen 10A0002A10 of alloy X750 irradiated to $0.31 \mathrm{dpa}$. .55

Figure 5-23: Overview of IASCC CGR test for specimen 10A0002B03 of alloy X-750 irradiated to 1.44 dpa. .56

Figure 5-24: In-situ fatigue pre-cracking steps for specimen 10A0002B03 of alloy X-750 irradiated to $1.44 \mathrm{dpa}$.

Figure 5-25: IASCC test transition to intergranular fracture for specimen 10A0002B03 of alloy $\mathrm{X}-750$ irradiated to $1.44 \mathrm{dpa}$. .58

Figure 5-26: Final transition to intergranular fracture and initial constant $\mathrm{K}$ for specimen 10A0002B03 of alloy X-750 irradiated to $1.44 \mathrm{dpa}$.

Figure 5-27: First transition from NWC to HWC in IASCC test for specimen 10A0002B03 of alloy X-750 irradiated to $1.44 \mathrm{dpa}$.

Figure 5-28: IASCC CGR test with -dK/da under NWC for specimen 10A0002B03 of alloy X750 irradiated to 1.44 dpa.

Figure 5-29: Applied K trajectory overlaid on IASCC CGR plot under -dK/da for specimen 10A0002B03 of alloy X-750 irradiated to $1.44 \mathrm{dpa}$.

Figure 5-30: Crack front straightening during IASCC CGR test of specimen 10A0002B03 of alloy X-750 irradiated to $1.44 \mathrm{dpa}$.

Figure 5-31: IASCC CGR during high pressure pump malfunction for specimen 10A0002B03 of alloy X-750 irradiated to $1.44 \mathrm{dpa}$.

Figure 5-32: IASCC CGR following return to NWC test conditions for specimen 10A0002B03 of alloy X-750 irradiated to $1.44 \mathrm{dpa}$. 
Figure 5-33: Final steps of IASCC CGR test for specimen 10A0002B03 of alloy X-750 irradiated to $1.44 \mathrm{dpa}$.

Figure 5-34: SEM micrograph of fracture surface for specimen 10A0002B03 of alloy X-750 irradiated to $1.44 \mathrm{dpa}$.

Figure 5-35: IASCC CGR test overview for specimen 10A0002B09 of alloy X-750 irradiated to $1.54 \mathrm{dpa}$.

Figure 5-36: In-situ pre-fatigue steps for specimen 10A0002B09 of alloy X-750 irradiated to 1.54 dpa.

Figure 5-37: IASCC transition to intergranular fracture for specimen 10A0002B09 of alloy X-750 irradiated to 1.54 dpa.

Figure 5-38: Initial IASCC CGR response at constant K for NWC and HWC in specimen 10A0002B09 of alloy X-750 irradiated to $1.54 \mathrm{dpa}$.

Figure 5-39: IASCC CGR test at constant K under HWC and NWC for specimen 10A0002B09 of alloy X-750 irradiated to $1.54 \mathrm{dpa}$.

Figure 5-40: IASCC CGR response during and after periods of gentle cycling to straighten the crack front for specimen 10A0002B09 of alloy X-750 irradiated to $1.54 \mathrm{dpa}$.

Figure 5-41: IASCC CGR response under HWC at higher $\mathrm{K}$ and gentle cycling for specimen 10A0002B03 of alloy X-750 irradiated to $1.54 \mathrm{dpa}$.

Figure 5-42: Final steps of IASCC CGR test for specimen 10A0002B03 of alloy X-750 irradiated to $1.54 \mathrm{dpa}$.

Figure 5-43: SEM micrograph of fracture surface for specimen 10A0002B09 of alloy X-750 irradiated to $1.54 \mathrm{dpa}$.

Figure 5-44: IASCC CGR test overview for specimen 10A0001B02 of XM-19 irradiated to 0.29 dpa.

Figure 5-45: In-situ fatigue pre-cracking for IASCC CGR test of specimen 10A0001B02 of XM19 irradiated to $0.29 \mathrm{dpa}$.

Figure 5-46: IASCC CGR response under NWC and constant K for specimen 10A0001B02 of XM-19 irradiated to 0.29 dpa.

Figure 5-47: IASCC CGR response under HWC and constant $\mathrm{K}$ for specimen 10A0001B02 of XM-19 irradiated to 0.29 dpa.

Figure 5-48: SEM micrograph of fracture surface for specimen 10A0001B02 of XM-19 irradiated to $0.29 \mathrm{dpa}$; IASCC CGR test end is marked by lower green line. .72

Figure 5-49: IASCC CGR test overview for specimen 10A0001B03 of XM-19 irradiated to 0.29 dpa.

Figure 5-50: In-situ pre-fatigue for IASCC test of specimen 10A0001B03 of XM-19 irradiated to $0.29 \mathrm{dpa}$.

Figure 5-51: Transition to intergranular fracture for IASCC test of specimen 10A0001B03 of XM-19 irradiated to 0.29 dpa.

Figure 5-52: IASCC CGR response under constant $\mathrm{K}$ and $\mathrm{HWC}$ and NWC with periods of gentle cycling for specimen 10A0001B03 of XM-19 irradiated to $0.29 \mathrm{dpa}$. 
Figure 5-53: IASCC CGR response under constant $\mathrm{K}$ and NWC with sulfate addition for specimen 10A0001B03 of XM-19 irradiated to $0.29 \mathrm{dpa}$.

Figure 5-54: IASCC CGR steady-state response under sustained constant K, and NWC and HWC for specimen 10A0001B03 of XM-19 irradiated to $0.29 \mathrm{dpa}$.

Figure 5-55: IASCC CGR response under gentle cycling followed by constant $\mathrm{K}$ under NWC for specimen 10A0001B03 of XM-19 irradiated to 0.29 dpa.

Figure 5-56: IASCC CGR response under constant $\mathrm{K}$ for return to $\mathrm{HWC}$, and final steps for specimen 10A0001B03 of XM-19 irradiated to 0.29 dpa.

Figure 5-57: SEM micrograph showing fracture surface for specimen 10A0001B03 of XM-19 irradiated to $0.29 \mathrm{dpa}$.

Figure 5-58: IASCC CGR test overview for specimen 10A0001B07 of XM-19 irradiated to 1.32 dpa.

Figure 5-59: In-situ pre-fatigue response for specimen 10A0001B07 of XM-19 irradiated to 1.32 dpa.

Figure 5-60: Transition to intergranular fracture for specimen 10A0001B07 of XM-19 irradiated to $1.32 \mathrm{dpa}$.

Figure 5-61: IASCC CGR response under constant K and transitions to HWC and back to NWC for specimen 10A0001B07 of XM-19 irradiated to $1.32 \mathrm{dpa}$.

Figure 5-62: IASCC CGR response under constant $\mathrm{K}$ with second transitions to HWC and back to NWC for specimen 10A0001B07 of XM-19 irradiated to $1.32 \mathrm{dpa}$.

Figure 5-63: Crack front straightening for specimen 10A0001B07 of XM-19 irradiated to 1.32 dpa.

Figure 5-64: Removal of sulfate and initiation of falling K for specimen 10A0001B07 of XM-19 irradiated to $1.32 \mathrm{dpa}$.

Figure 5-65: Continuation of falling $\mathrm{K}$ and transition to constant $\mathrm{K}$ for specimen 10A0001B07 of XM-19 irradiated to 1.32 dpa.

Figure 5-66: Trajectory of falling K and effect on IASCC CGR for specimen 10A0001B07 of XM-19 irradiated to 1.32 dpa.

Figure 5-67: IASCC CGR response following -dK/da under NWC and HWC for specimen 10A0001B07 of XM-19 irradiated to $1.32 \mathrm{dpa}$

Figure 5-68: Closeup of region immediately following $-\mathrm{dK} / \mathrm{da}$ for specimen 10A0001B07 of XM19 irradiated to $1.32 \mathrm{dpa}$.

Figure 5-69: Final steps of IASCC CGR test for specimen 10A0001B07 of XM-19 irradiated to $1.32 \mathrm{dpa}$.

Figure 5-70: SEM micrograph showing fracture surface of specimen 10A0001B07 of XM-19 irradiated to $1.32 \mathrm{dpa}$.

Figure 5-71: Side view of specimen 10A0001B07 of XM-19 irradiated to 1.32 dpa showing outof-plane cracking.

Figure 5-72: IASCC CGR test overview for specimen 10A0001D02 of XM-19 irradiated to 1.44 dpa. 
Figure 5-73: In-situ fatigue pre-crack response for specimen 10A0001D02 of XM-19 irradiated to $1.44 \mathrm{dpa}$.

Figure 5-74: Transition to intergranular fracture for IASCC CGR test of specimen 10A0001D02 of XM-19 irradiated to $1.44 \mathrm{dpa}$.

Figure 5-75: IASCC CGR test under constant $\mathrm{K}$ with transition to HWC and back to NWC for specimen 10A0001D02 of XM-19 irradiated to $1.44 \mathrm{dpa}$.

Figure 5-76: SEM micrograph showing fracture surface of specimen 10A0001D02 of XM-19 irradiated to $1.44 \mathrm{dpa}$.

Figure 5-77: Crack length vs. time for 0.5TCT specimen of alloy X-750 in the HTH condition tested in $288^{\circ} \mathrm{C}$ water [3] [16] [15] showing very similar behavior to irradiated alloy $\mathrm{X}-750$ in this project.

Figure 5-78: Crack length vs. time for 0.5TCT specimen of alloy X-750 in the HTH condition tested in $288^{\circ} \mathrm{C}$ water [3] [16] [15] showing very similar behavior to irradiated alloy $\mathrm{X}-750$ in this project.

Figure 5-79: Crack length vs. time for 0.5TCT specimen of XM-19 (Nitronic 50) with 21\% cold work tested in $288^{\circ} \mathrm{C}$ water [15] [14] showing similar behavior to irradiated XM-19 in this project.

Figure 5-80: Crack length vs. time for 0.5TCT specimen of XM-19 (Nitronic 50) with $19 \%$ cold work tested in $288^{\circ} \mathrm{C}$ water [15] [14] showing similar behavior to irradiated XM-19 in this project.

Figure 5-81: Crack growth rate vs. stress intensity factor $(\mathrm{K})$ for unirradiated alloy X-750, separated into high and low ECP data [15] [14]....

Figure 5-82: Crack growth rate vs. stress intensity factor $(\mathrm{K})$ for all irradiated alloys X-750 and XM-19, separated into high and low ECP data.

Figure 5-83: Crack growth rate vs. stress intensity factor $(\mathrm{K})$ for high-quality irradiated alloys $\mathrm{X}$ 750 and XM-19, separated into high and low ECP data.

Figure 5-84: Crack growth rate benefit of shifting from high to low ECP of unirradiated alloy X750 , separated into 0 and $10 \mathrm{ppb}$ sulfate data [15] [14]...... 96

Figure 5-85: Crack growth rate benefit of shifting from high to low ECP of irradiated alloy X-750 in this project.

Figure 5-86: Crack growth rate benefit of shifting from high to low ECP of irradiated XM-19 in this project.

Figure 5-87: Crack growth rate benefit of shifting from pure water to 10 or $30 \mathrm{ppb}$ sulfate in unirradiated alloy X-750 [15] [14].

Figure 5-88: Crack growth rate benefit of shifting from pure water to 10 or $30 \mathrm{ppb}$ sulfate in irradiated alloy $\mathrm{X}-750$ in this project.

Figure 5-89: The dependency of stress intensity factor (K) on SCC growth rate in individual specimens (upper plots), and a screened database (lower plot) of unirradiated alloy X750 [15] [14].

Figure 5-90: The dependency of stress intensity factor $(\mathrm{K})$ on SCC growth rate in individual specimens of unirradiated XM-19 [15] [14]. 
Figure 5-91: SCC growth rate vs. stress intensity factor (K) for irradiated alloys X-750 and XM-

19 , showing the effect of higher vs. lower fluence at high ECP in this project.

Figure 5-92: SCC growth rate vs. stress intensity factor (K) for alloys X-750 and XM-19, showing the effect of higher vs. lower fluence at low ECP.

Figure 6-1: STEM-EDS image of HAGB in unirradiated alloy X-750.

Figure 6-2: High magnification STEM-EDS image of HAGB in unirradiated alloy X-750.

Figure 6-3: TEM diffraction pattern of the matrix containing $\gamma^{\prime}$ precipitates of unirradiated alloy $\mathrm{X}-750$, taken along the [011] zone axis. The superlattice reflections from the $\gamma^{\prime}$ precipitates are indicated by dashed circles (left) and arrows (right).

Figure 6-4: STEM-EDS image of unirradiated XM-19 showing coarse (Nb, Mo)-rich nitride precipitates.

Figure 6-5: TEM rel-rod DF images showing the evolution of Frank loops in alloys X-750 and XM-19 irradiated to three different fluences.

Figure 6-6: Size distributions and number densities of the Frank loops in alloys X-750 and XM19 irradiated to three different fluences (doses).

Figure 6-7: STEM-EDS map of the matrix: (a) STEM BF image, (b)-(h) elemental maps of Fe, $\mathrm{Cr}, \mathrm{Ni}, \mathrm{Ti}, \mathrm{Al}, \mathrm{V}$, and $\mathrm{Nb}$, respectively.

Figure 6-8: TEM diffraction pattern of the matrix containing $\gamma^{\prime}$ precipitates of the high fluence alloy X-750, taken along the [011] zone axis. The superlattice reflections from the $\gamma^{\prime}$ precipitates are indicated by dashed circles (left) and arrows (right). 108

Figure 6-9: Comparison of the relative intensity of the superlattice reflections from the $\gamma^{\prime}$ precipitates in alloy X-750 at different irradiation conditions. The intensity profiles were obtained from diffraction patterns and have been adjusted so that the background signal and widths of the matrix reflection peaks are similar. 108 


\section{TABLES}

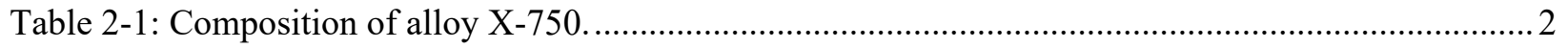

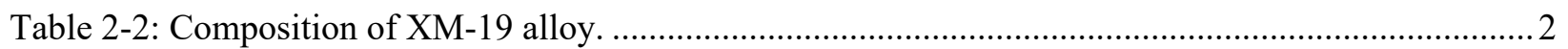

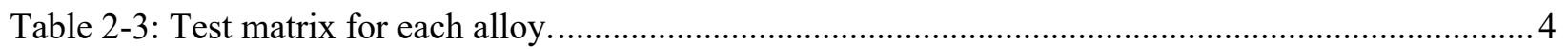

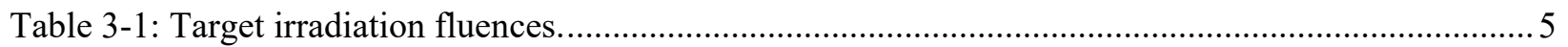

Table 3-2: EPRI-1 alloy X-750 estimated irradiation temperature and fluence. ........................................ 11

Table 3-3: EPRI-1 XM-19 estimated irradiation temperature and fluence................................................ 11

Table 3-4: EPRI-2 alloy X-750 estimated fluence and irradiation temperature. ...................................... 12

Table 3-5: EPRI-2 XM-19 estimated fluence and irradiation temperature.............................................. 12

Table 3-6: EPRI-3 alloy X-750 estimated fluence and irradiation temperature. ..................................... 13

Table 3-7: EPRI-3 XM-19 estimated fluence and irradiation temperature............................................. 13

Table 4-1: Irradiated and baseline tensile properties for alloy X-750 at $288^{\circ} \mathrm{C}$. .................................... 16

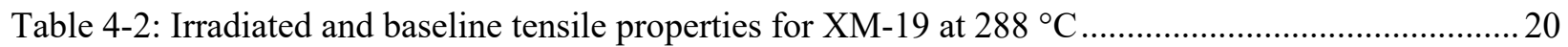

Table 4-3: Pre-fatigue parameters for alloy X-750 fracture toughness specimens. .................................23

Table 4-4: Pre-fatigue parameters for XM-19 fracture toughness specimens. ........................................24

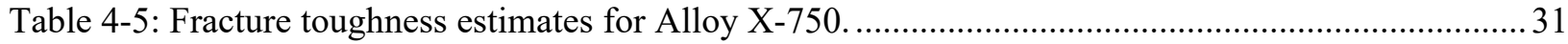

Table 4-6: Fracture toughness estimates for XM-19 (asterisk indicates $\mathrm{J}_{\mathrm{IC}} / \mathrm{K}_{\mathrm{IC}}$ ) ................................. 37

Table 4-7: Table of ASTM validity checks for all fracture toughness tests [4] .......................................38

Table 5-1: Constant K IASCC CGRs and conditions for specimen 10A0002A08 of alloy X-750 irradiated to $0.30 \mathrm{dpa}$; blue highlights indicate higher confidence data. .43

Table 5-2: Constant K IASCC CGR and related conditions for specimen 10A0002A10 of alloy X750 irradiated to $0.31 \mathrm{dpa}$. .50

Table 5-3: Constant K IASCC CGR and related conditions for specimen 10A0002B03 of alloy X750 irradiated to $1.44 \mathrm{dpa}$; blue highlights indicate higher confidence data. .58

Table 5-4: Constant K IASCC CGR and related conditions for specimen 10A0002B09 of alloy X750 irradiated to $1.54 \mathrm{dpa}$; blue highlights indicate higher confidence data.

Table 5-5: Constant K IASCC CGR and related conditions for specimen 10A0001B02 of XM-19 irradiated to 0.29 dpa.

Table 5-6: Constant K IASCC CGR and related conditions for specimen 10A0001B03 of XM-19 irradiated to $0.29 \mathrm{dpa}$; blue highlights indicate higher confidence data.

Table 5-7: Constant K IASCC CGR and related conditions for specimen 10A0001B07 of XM-19 irradiated to $1.32 \mathrm{dpa}$.

Table 5-8: Constant K IASCC CGR and related conditions for specimen 10A0001D02 of XM-19 irradiated to 1.44 dpa. 88

Table 5-9: Growth rate benefit of HWC evaluated using different subsets of data-calculated as [growth rate at high ECP] divided by [growth rate at low ECP] 
Table 6-1: Quantitative statistical data for the average size and number density of the Frank loops irradiated to three different fluences. 


\section{ACRONYMS}

ASTM American Society for Testing of Materials

ATR Advanced Test Reactor

BF Bright Field

BWR Boiling Water Reactor

CFT Center Flux Trap

CGR Crack Growth Rate

CT Compact Tension

CW Cold Worked

DCPD Direct Current Potential Drop

DF Dark Field

dpa Displacements Per Atom

ECP Electrochemical Corrosion Potential

EDS Energy Dispersive Spectroscopy

EPRI Electric Power Research Institute

FIB Focused Ion Beam

FT Fracture Toughness

GE-GRC GE-Global Research Center

HAGB High Angle Grain Boundary

HWC Hydrogen Water Chemistry

IASCC Irradiation Assisted Stress Corrosion Cracking

INL Idaho National Laboratory

LVDT Linear Variable Displacement Transducer

MCNP Monte-Carlo N-Particle

NSUF National Scientific User Facility

NWC Normal Water Chemistry

SCC Stress Corrosion Cracking

SEM Scanning Electron Microscope

STEM Scanning Transmission Electron Microscope

TEM Transmission Electron Microscope 


\title{
SI - U.S. UNIT CONVERSIONS
}

\author{
$1 \mathrm{~mm}=0.0394$ in \\ $1 \mathrm{MPa}=0.145 \mathrm{ksi}$ \\ $1 \mathrm{~kJ} / \mathrm{m}^{2}(\mathrm{kPa}-\mathrm{m})=5.71 \mathrm{psi}$-in \\ $1 \mathrm{MPa} \sqrt{\mathrm{m}}=0.910 \mathrm{ksi} \sqrt{\mathrm{in}}$ \\ $288^{\circ} \mathrm{C}=550^{\circ} \mathrm{F}$
}




\section{Introduction}

The Advanced Test Reactor (ATR) National Scientific User Facility (ATR NSUF), established in April 2007 and originally located exclusively at Idaho National Laboratory (INL), was created to facilitate and support world-class nuclear R\&D for academia, the commercial nuclear power industry, and other national laboratories engaged in nuclear power R\&D. The ATR-NSUF also sought to promote cooperative research with industry by jointly developing infrastructure and executing programs that address issues that have wide applicability across the nuclear industry. Since its inception, the ATRNSUF has expanded to include multiple partner facilities, and, in 2014, evolved into what is now simply called NSUF, a name change reflecting the multi-facility nature of the program. This pilot project with the Electric Power Research Institute (EPRI) was initiated in 2009.

Although extensively used for research by the Department of Energy and the National Nuclear Security Administration, the ATR had not been readily available for use by the commercial nuclear power sector prior to 2007. Industry thus had little experience with using the ATR and its post-irradiation examination support facilities. The EPRI Pilot Project, described below, provides a test case in which experimental and administrative protocols can be developed while generating data needed by industry.

EPRI conducts R\&D on the generation, delivery, and use of electricity for the benefit of the public. An independent and nonprofit organization, EPRI brings together its scientists and engineers, as well as experts from academia and industry, to help address challenges in electricity, including reliability, efficiency, health, safety, and the environment.

As a means of establishing a basis for development and execution of joint NSUF-industry programs, EPRI and the Department of Energy agreed to develop a pilot program involving shared costs and responsibilities. In addition to providing data, the pilot program (referred to as the EPRI Pilot Project) is designed to:

- Develop the administrative protocols for cooperative research, such as cooperative agreements and funding.

- Develop the research capability and staffing required to address future R\&D needs.

- Develop a level of confidence in generating relevant data, particularly irradiation assisted stress corrosion crack (IASCC) growth rate data.

Discussions between NSUF and EPRI identified investigation of the fracture toughness (FT) and IASCC growth rates of irradiated high-strength alloys used for boiling water reactor (BWR) repair hardware as an area of mutual interest for an initial project; very little IASCC and irradiated FT data exist for alloy X-750 (a Ni-based alloy) and XM-19 (nitrogen strengthened austenitic stainless steel) at the exposure levels of interest (up to $1 \times 10^{21} \mathrm{n} / \mathrm{cm}^{2}$ ). Therefore, the focus of the EPRI Pilot Project is on the irradiation and characterization of these alloys in both unirradiated (baseline) and irradiated states, and is being conducted in three phases. Phase I (CRADA 09-CR-02) and Phase II (CRADA10-CR-13) are complete. Phase 1 [1] fabricated the EPRI specimens from materials provided by EPRI and established the baseline FT and crack growth rates (CGRs) of unirradiated material. Phase 2 [2] designed and fabricated the specimen holders and performed a safety analysis on the irradiation experiment to meet EPRI objectives for the irradiation of tensile and compact tension specimens in the center flux trap of ATR utilizing Loop 2A. The current phase of this project, Phase III (CRADA 12-CR-06), is the subject of this report and includes irradiation and post irradiation examination of the EPRI specimens delivered by EPRI to NSUF in Phases I and II. 


\section{Materials, Specimens, and Test Matrix}

\subsection{Alloy X-750}

Several sections of alloy X-750 (Heat \#2750-5-7656) were provided to INL by GE-Global Research Center (GE-GRC). These sections were removed by GE-GRC from a spare upper support bracket that was purchased from Southern Co. by EPRI. Details of the sectioning of this piece and the machining of specimens may be found in the appendix of the Phase I report [1]. The material was delivered in the HTH condition: which consists of a $35 \%$ minimum reduction hot roll ${ }^{\text {a }}$; solution anneal at $1107{ }^{\circ} \mathrm{C}$ for 1 hour and rapid cool; and aging at $704{ }^{\circ} \mathrm{C}$ for 20 hours and air cool. The composition for this material is given in Table 2-1, and additional microstructural details may be found in [3]. Note that one element is unreadable on the supplied material test report and is shown here as a question mark. Based on comparable specification sheets for X-750, it is likely that this unknown element is $\mathrm{Nb}$.

Table 2-1: Composition of alloy X-750.

\begin{tabular}{|c|c|c|c|c|c|c|c|c|c|c|c|c|c|c|}
\hline Al & C & $\begin{array}{c}\text { Nb+ } \\
\text { Ta }\end{array}$ & Co & Cr & Cu & Fe & Mg & Ni & P & S & Si & Ti & Ta & $?$ \\
\hline $\mathbf{0 . 7 7}$ & $\mathbf{0 . 0 4}$ & $\mathbf{0 . 9 9}$ & $\mathbf{0 . 7 2 6}$ & $\mathbf{1 4 . 9 9}$ & $\mathbf{0 . 0 1 5 1}$ & $\mathbf{7 . 8}$ & $\mathbf{0 . 1 9 7}$ & $\mathbf{7 0 . 8}$ & $<0.005$ & $\mathbf{0 . 0 0 2}$ & $\mathbf{0 . 2 5}$ & $\mathbf{2 . 4 2}$ & $<0.01$ & $\mathbf{0 . 9 7}$ \\
\hline
\end{tabular}

\section{$2.2 \quad \mathrm{XM}-19$}

Two sections of alloy XM-19 (Heat \#A17509-BD3) were provided to INL by GE-GRC. One of the sections was provided in the annealed condition and the other was cold rolled in a large rolling mill at GE-GRC to induce a total of $19.3 \%$ cold work in two passes. It was decided by the project team to use the $19.3 \%$ cold worked $(\mathrm{CW})$ material for the baseline testing performed under phase I of the project [1] to allow comparison as to the effect of neutron embrittlement on the annealed material under Phase III. Similar to what was observed in [1], it is expected that neutron embrittlement will increase the yield strength of the material and reduce its fracture toughness. The material composition is shown here in Table 2-2; further details on the microstructure of the annealed alloy XM-19 may be found in [3].

Table 2-2: Composition of XM-19 alloy.

\begin{tabular}{|c|c|c|c|c|c|c|c|c|c|c|c|c|c|}
\hline $\mathrm{Mo}$ & $\mathrm{C}$ & $\mathrm{Nb}$ & $\mathrm{Co}$ & $\mathrm{Cr}$ & $\mathrm{Cu}$ & $\mathrm{Fe}$ & $\mathrm{Mn}$ & $\mathrm{Ni}$ & $\mathrm{P}$ & $\mathrm{S}$ & $\mathrm{Si}$ & $\mathrm{Ti}$ & $\mathrm{W}$ \\
\hline 2.17 & 0.027 & 0.20 & 0.016 & 20.76 & 0.06 & $\mathrm{Bal}$ & 4.59 & 12.82 & 0.014 & 0.006 & 0.39 & 0.001 & 0.02 \\
\hline
\end{tabular}

\subsection{Specimens for Mechanical Properties Testing}

\subsubsection{IASCC and Fracture Toughness}

Specimens to be irradiated and used for IASCC CGR testing as well as fracture toughness were American Society for Testing of Materials (ASTM) standard [4] specimens designated 0.4T-CT, meaning 0.4-inch (10.2-mm) thick, compact tension. The 0.4-inch $(10.2-\mathrm{mm})$ thickness was chosen to promote appropriate constraint for validity of fracture toughness and IASCC testing while minimizing size to the extent possible. For this project, all specimens were machined in the L-T orientation with respect to rolling direction, with integral side grooves at 5\% depth (per side) to help control crack tunneling. Figure 2-1 is a sketch of the 0.4T-CT specimen used for this project.

\footnotetext{
${ }^{a}$ Note that this step is typical of the HTH heat treatment, but not documented in the material certification for the material used in this project.
} 


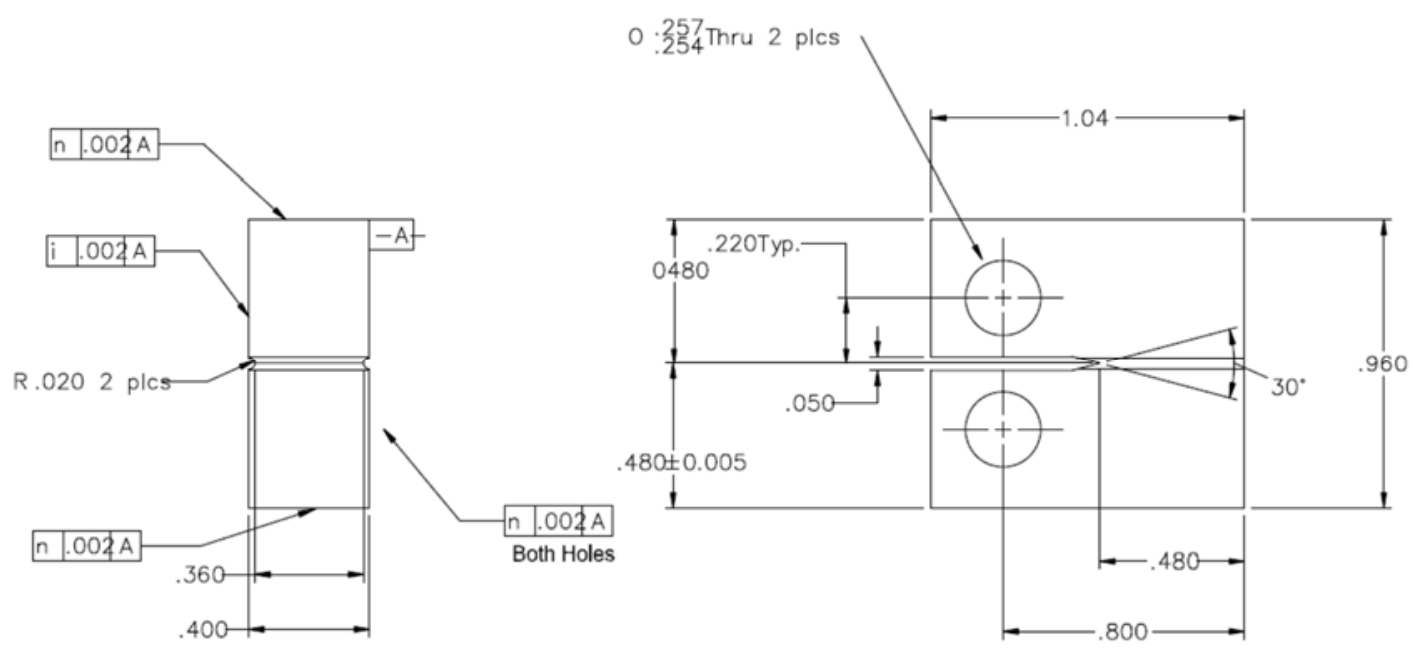

Figure 2-1: 0.4T-CT specimen for IASCC and fracture toughness (dimensions in inches).

\subsubsection{Tensile Specimens}

Tensile specimens are full sized in order to avoid any uncertainty associated with size effects, etc. They are machined with the long axis coincident with the L (rolling) direction in the original plate according to ASTM Standard E-8 [5] into a round "dog-bone" geometry with a 6.35-mm (0.25-in) gage diameter and a $31.75-\mathrm{mm}(1.25$-in) reduced section length. Figure 2-2 is a sketch of the tensile specimens used for this project.
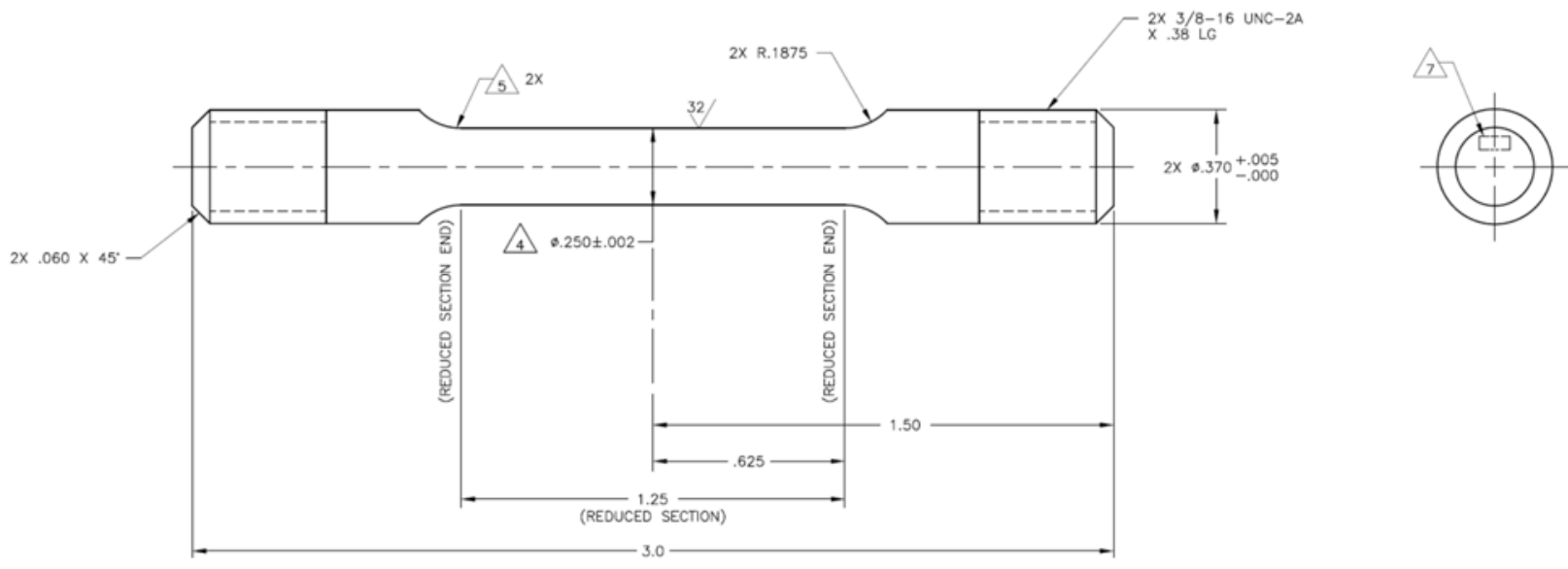

Figure 2-2: Standard tensile specimen (dimensions in inches).

\subsection{Test Matrix}

The test matrix for this project originally included testing to ascertain the existence of an environmental effect on fracture toughness, but these tests were considered to be of secondary importance to IASCC, FT, and tensile testing, and were abandoned in the interest of time. Additionally, results of IASCC testing on medium and high fluence specimens suggested that conducting IASCC tests on the lowest fluence specimens would not provide additional benefit to the project. Therefore, IASCC tests 
were not conducted on the lowest fluence specimens. There are a considerable number of backup specimens that were irradiated and will be available to NSUF users following the completion of this project. Table 2-3 shows the matrix of each alloy included in this project (total number of specimens are double that shown in Table 2-3, since there are two alloys). Here, NWC refers to BWR Normal Water Chemistry and HWC refers to BWR Hydrogen Water Chemistry.

Table 2-3: Test matrix for each alloy.

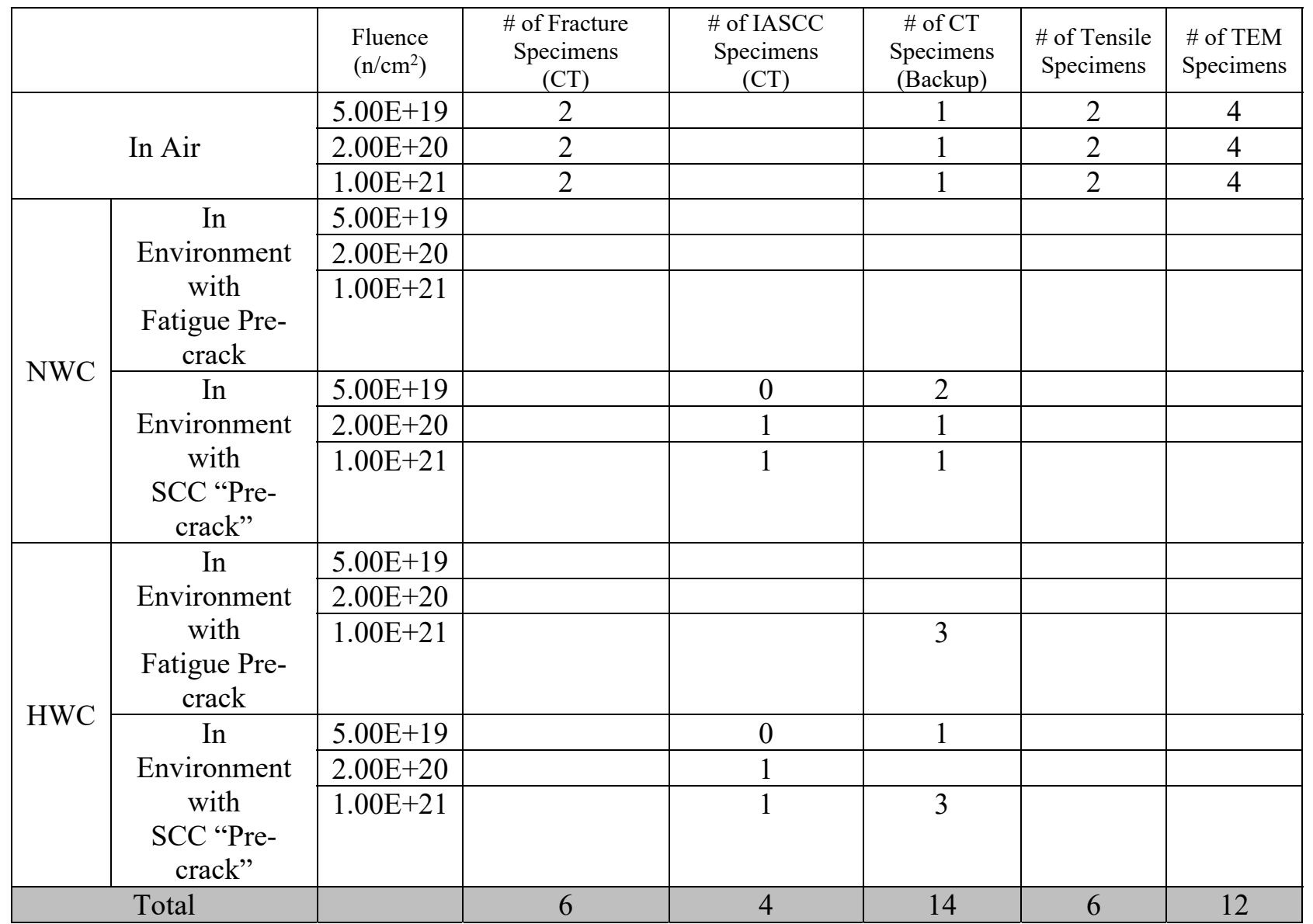

\section{Irradiation Details}

This project was the first civilian (non-Navy) irradiation experiment to utilize the ATR center flux trap (CFT) and associated water loop. The CFT has a nominal thermal flux $(\mathrm{E}<1.0 \mathrm{MeV})$ of $4.4 \times 10^{14}$ $\mathrm{n} / \mathrm{cm}^{2}$-s and a nominal fast flux $(\mathrm{E}>1.0 \mathrm{MeV})$ of $9.7 \times 10^{13} \mathrm{n} / \mathrm{cm}^{2}$-s with the reactor operating at its typical power of approximately $110 \mathrm{MW}_{\text {th }}$. This fast flux provides very rapid neutron damage, enabling high fluence irradiation studies of structural materials and fuels to be completed in a matter of years, rather than the decades it would take to accumulate equivalent damage in typical commercial surveillance capsules. A pressurized, chemistry controlled water loop was re-installed in the CFT in the $2007-2009$ timeframe, enabling the design of experiments that utilized flowing water as a temperature control mechanism. This loop is referred to as Loop-2A. 
Although Loop 2A and the CFT had been previously utilized by the U.S. Navy, details on the operations and resident water chemistry are classified. Thus, the EPRI Pilot Project represented a learning opportunity for NSUF.

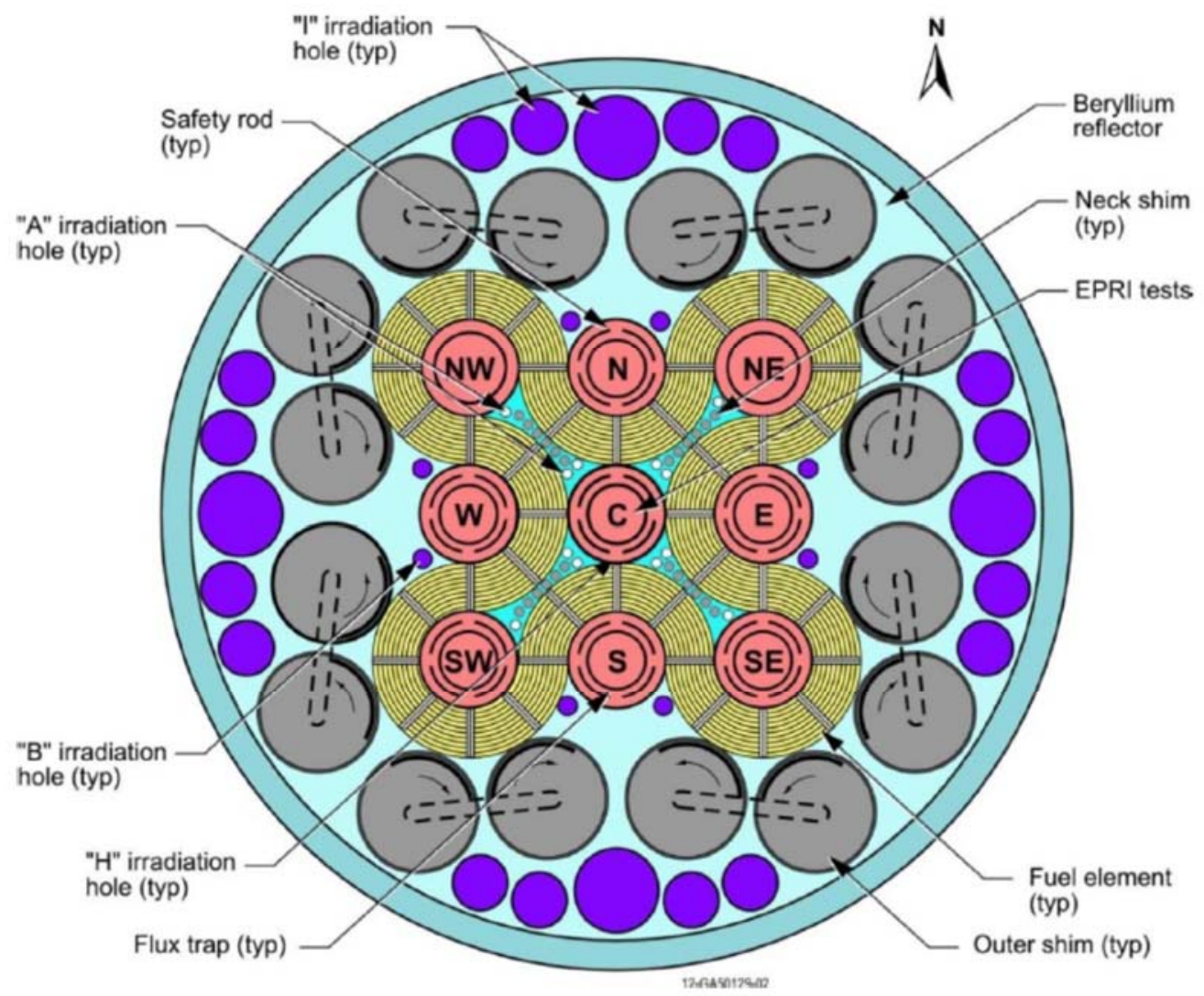

Figure 3-1: ATR cross section, CFT is labeled "C".

The pilot project targeted three fast $(\mathrm{E}>1 \mathrm{MeV})$ target fluences of $5 \times 10^{19} \mathrm{n} / \mathrm{cm}^{2}, 2.0 \times 10^{20} \mathrm{n} / \mathrm{cm}^{2}$, and $1.0 \times 10^{21} \mathrm{n} / \mathrm{cm}^{2}$. Each irradiation target had its own capsule train. Table 3-1 shows the experiment designators and target fluences.

Table 3-1: Target irradiation fluences.

\begin{tabular}{|c|c|c|}
\hline $\begin{array}{c}\text { Specimen Holder } \\
\text { (Experiment) ID }\end{array}$ & $\begin{array}{c}\text { ATR Insertion Dates and } \\
\text { Cycles }\end{array}$ & $\begin{array}{c}\text { Target Fluence (E> } \\
1.0 \mathrm{MeV})\end{array}$ \\
\hline EPRI-1 & February 2015(157C) & $5.0 \times 10^{19} \mathrm{n} / \mathrm{cm}^{2}$ \\
\hline EPRI-2 & March 2013 (153B) & $2.0 \times 10^{20} \mathrm{n} / \mathrm{cm}^{2}$ \\
\hline EPRI-3 & $\begin{array}{c}\text { February 2014, February } 2016 \\
(155 \mathrm{~B}, 158 \mathrm{~B})\end{array}$ & $1.0 \times 10^{21} \mathrm{n} / \mathrm{cm}^{2}$ \\
\hline
\end{tabular}

\subsection{Irradiation Experiment Capsules and Specimen Layout}

The specimens were irradiated in the ATR CFT, which uses chemistry controlled water coolant with a variable flow rate and water inlet temperature to control irradiation temperature. The irradiation capsule 
design (Figure 3-2) uses multiple sets of two 0.4T-CT specimens with approximately 0.25 -mm flow spacings between them and ASTM standard round, dog-bone tensile specimens stacked on top of each other inside a zirconium alloy holder; TEM discs are stacked inside a cavity that is drilled into a $0.4 \mathrm{~T}-\mathrm{CT}$ specimen blank (Figure 3-2). The overall test train consists of four of these zirconium alloy holders that are interlocked to form a $1.625-\mathrm{m}$ (64-in) long test train. The test train is located between $0.61 \mathrm{~m} \mathrm{(24} \mathrm{in.)}$ below the ATR core mid-plane and $1.02 \mathrm{~m}$ (40 in.) above the ATR core mid-plane in order to approximately center the primary irradiation targets (the EPRI specimens) across a relatively flat portion of the ATR flux profile. Figure 3-3 is a representation of the axial flux profile of the ATR. Specimen packages of interest were located within the experiment test train to coincide with the relatively "flat" portion of the axial flux profile between approximately -0.25 and $+0.25 \mathrm{~m}(-10$ and +10 inches $)$ of the center. Further details on capsule and experiment layout may be found in [2].

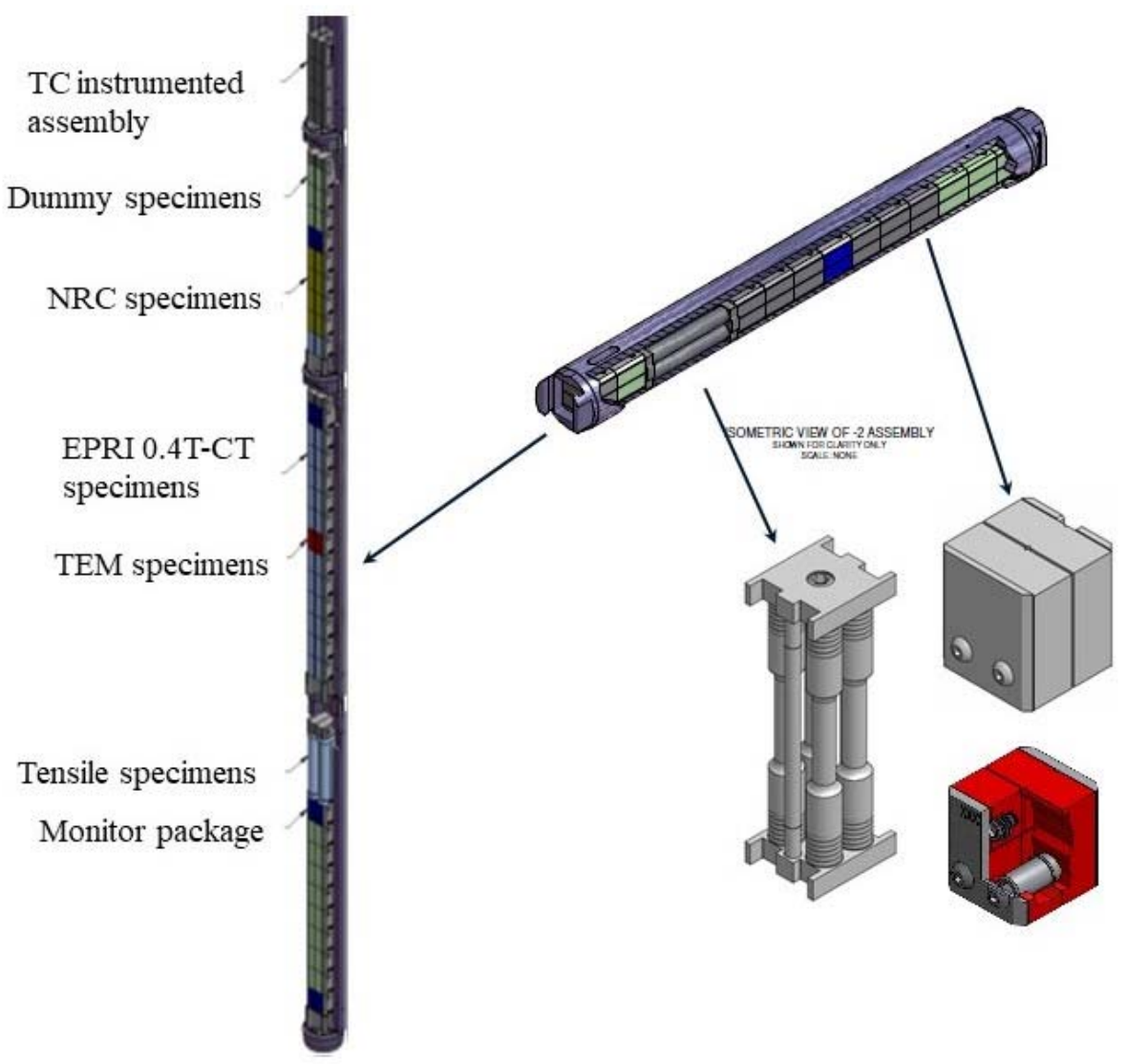

Figure 3-2: Experiment capsules and specimen stack-up. 


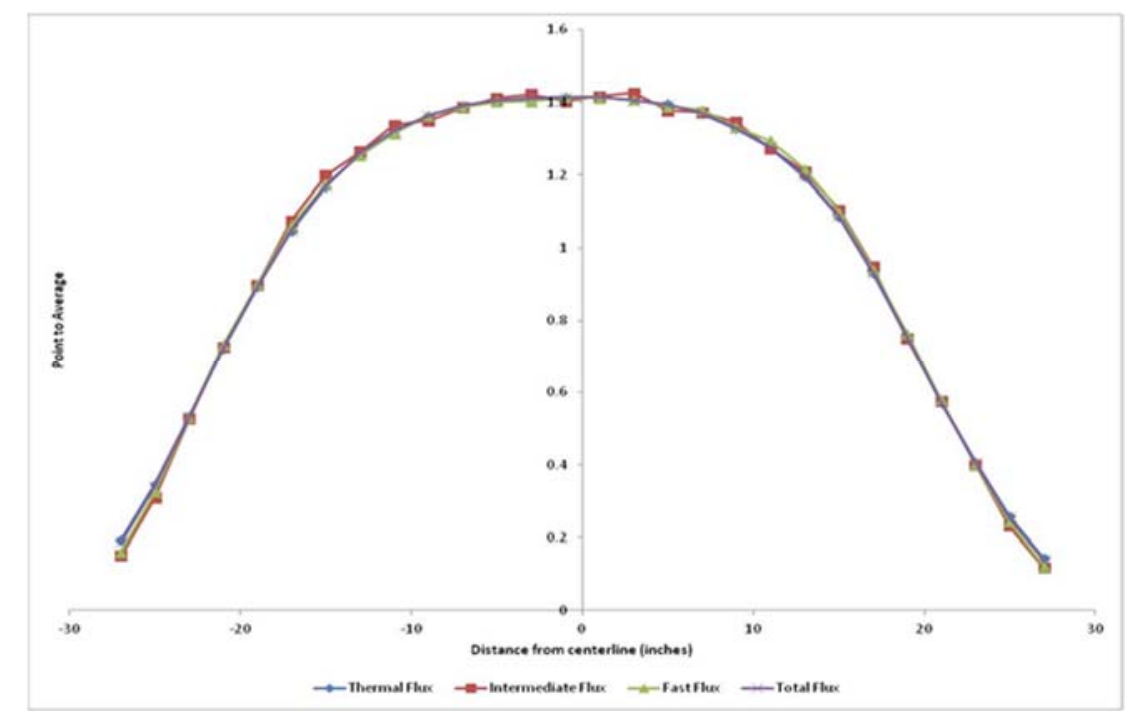

Figure 3-3: Generalized ATR axial flux profile (dimensions in inches).

\subsubsection{Flow Restriction Issue and Mitigation}

During irradiation of the very first experiment (EPRI-2) in the newly reactivated ATR Loop 2A, the pressure drop across the test train steadily increased during the cycle, indicating a coolant flow restriction which eventually required startup of a third loop pump to maintain flow. The cause of this pressure drop was determined to be a buildup of crud in the thin coolant flow channels between specimens in the specimen package located near the bottom of each capsule - an example of which is shown in Figure 3-4. It is assumed that this crud resulted from oxide stripping that occurred due to incompatibility of the coolant used with that previously employed in the loop. The experiment was subsequently redesigned to replace the bottom specimen in each holder with a cruciform spacer (Figure 3-5). These spacers were meant to reduce the flow restriction where the coolant exits the holder. During irradiation of the EPRI-3 experiment in cycle 155B (the subsequent irradiation experiment), the pressure drop improved but was still too high. The experiment was again redesigned prior to cycles 157C (EPRI-1 irradiation) and 158B (EPRI-3 second irradiation cycle) to include centering spacers between the holder and specimens (for the EPRI-1 irradiation) and to replace the entire lower stack of CT specimens by a flow-through spacer for the EPRI-3 second irradiation cycle (Figure 3-6 and Figure 3-7). The centering spacers were meant to reduce misalignment of coolant channels, which had led to the increased pressure drop observed in the previous EPRI experiments. Additionally, $\mathrm{LiOH}$ resin was placed in the loop ion exchange columns in order to increase $\mathrm{pH}$. These design modifications reduced the test train pressure drop (hence, improving cooling performance) to acceptable levels for the second cycle of the EPRI-3 experiment. 


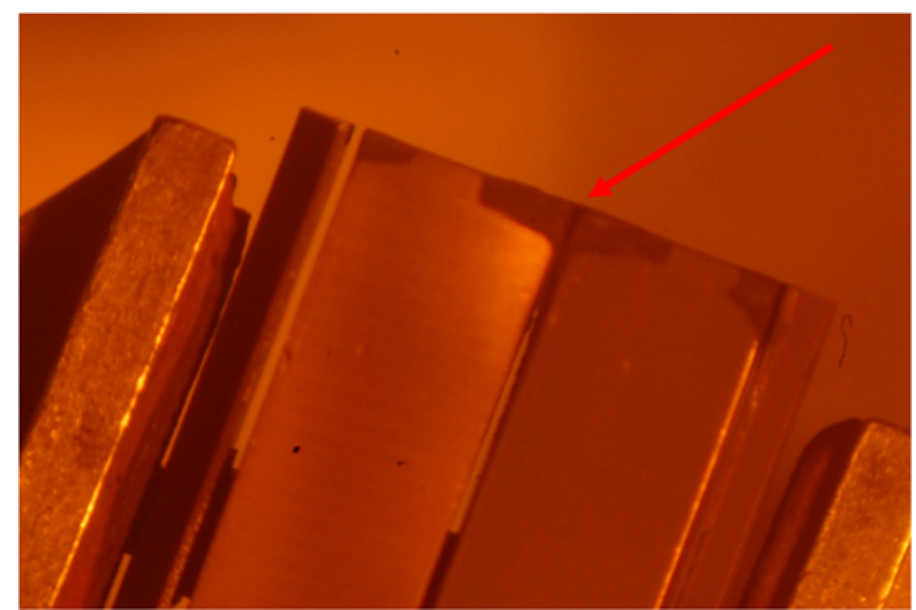

Figure 3-4: Photograph showing buildup of crud in specimen package flow channel.
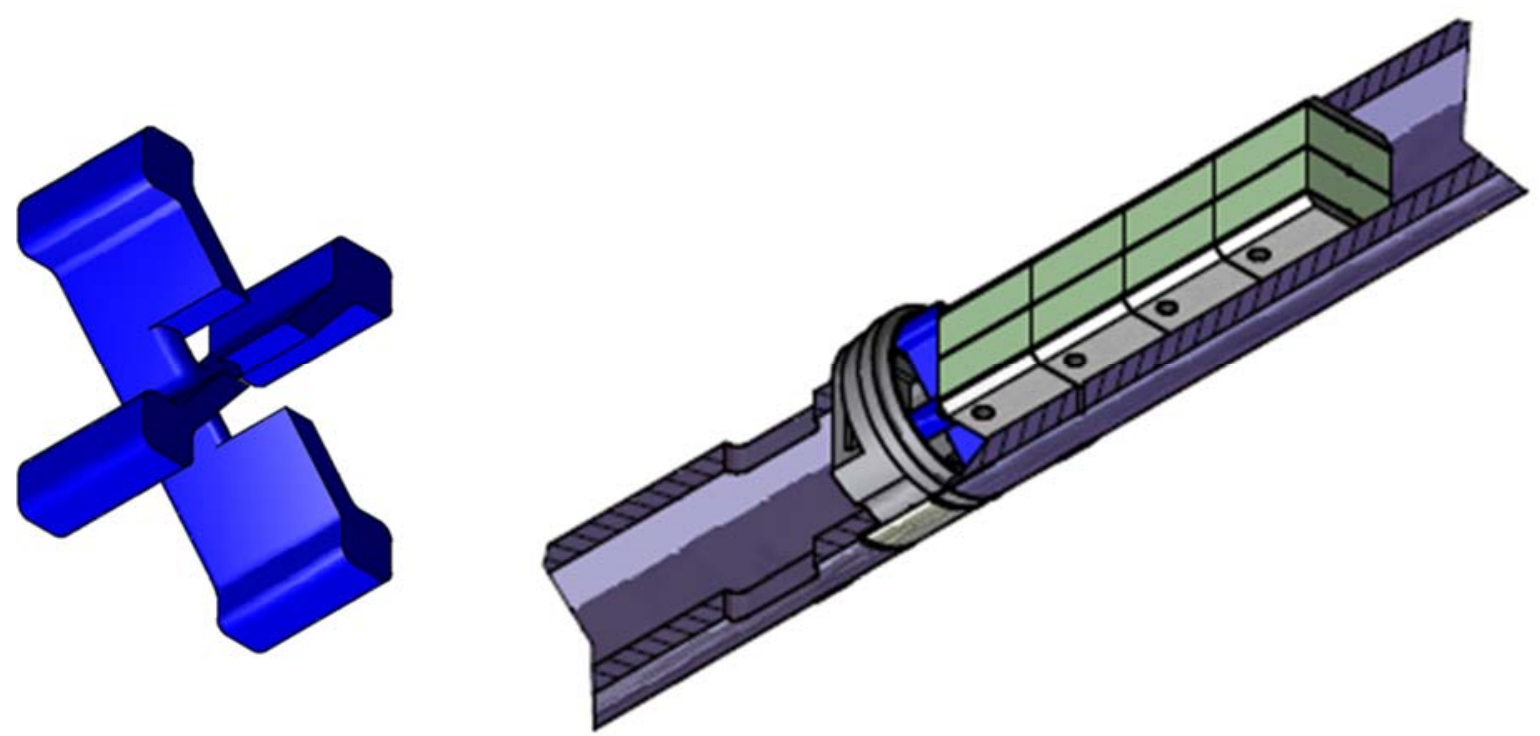

Figure 3-5: Cruciform offset spacer to mitigate flow restriction issue. 


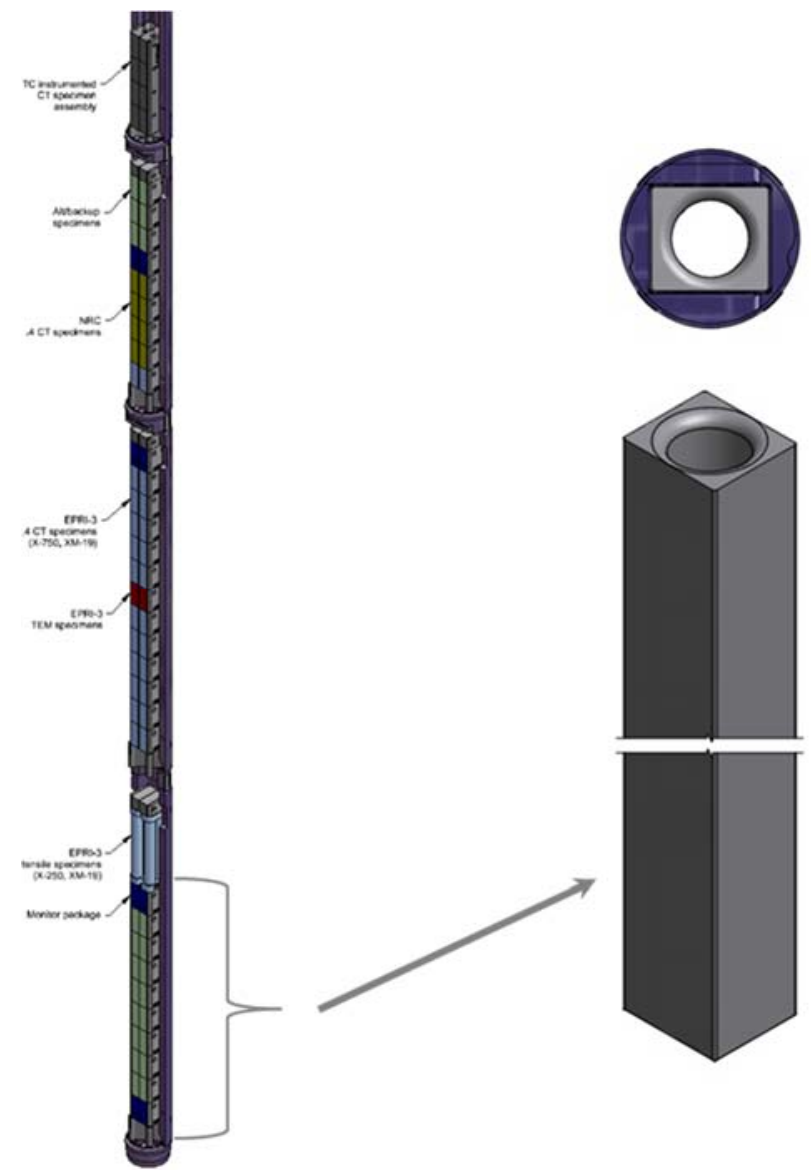

Figure 3-6: Additional flow restriction mitigation employed for EPRI-3-2. 

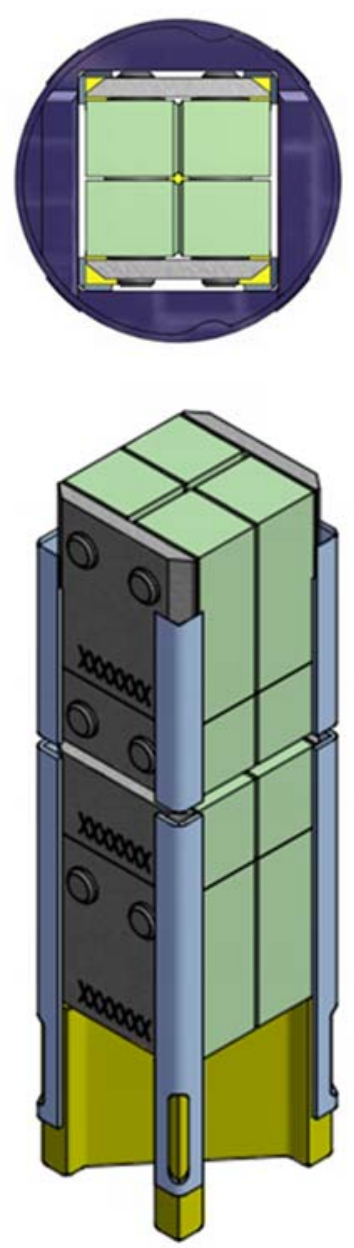

Figure 3-7: Centering spacers designed to prevent twisting of specimen packages.

\subsection{EPRI-1 Thermal and Neutronics}

The EPRI-1 experiment capsule was irradiated in ATR cycle 157C, a shortened cycle which was started on February 10, 2015, and ran at full power for just over five days, ending on February 15, 2015. The fast fluence target for this irradiation capsule was $5.0 \times 10^{19} \mathrm{n} / \mathrm{cm}^{2}$. Temperature was measured during the experiment utilizing thermocouples embedded in specimen blanks near the top of the experiment and packages of melt wires selected to span the target temperature of $288^{\circ} \mathrm{C}\left(550^{\circ} \mathrm{F}\right)$ from 239 to $327^{\circ} \mathrm{C}$. Individual specimen irradiation temperatures were estimated in the post irradiation as-run analysis using an ABAQUS model that was compared to thermocouple and melt wire indications. It is noted that temperatures are estimated to have exceeded the target temperature quite significantly as a result of the aforementioned flow blockage issue that was not completely solved when this experiment was irradiated. All melt wires were observed to have melted, which is consistent with the prediction that irradiation temperature exceeded $327^{\circ} \mathrm{C}$ for at least a short time. Accumulated fluence was also estimated using a Monte-Carlo N-Particle (MCNP) simulation. Table 3-2 shows estimated fluence as well as peak and average temperatures for the EPRI-1 alloy X-750 specimens of interest in this project, and Table 3-3 shows estimated fluence as well as peak and average cycle temperatures for the EPRI-1 XM-19 specimens of interest in this project. Yellow highlighted cells identify specimens that were used for fracture toughness testing in this project. Full details of these analyses are available in [6] and [7]. 
Table 3-2: EPRI-1 alloy X-750 estimated irradiation temperature and fluence.

\begin{tabular}{|c|c|c|c|c|c|}
\hline Package & Specimen ID & Fluence $\left(\mathrm{n} / \mathrm{cm}^{2}\right)$ & dpa (X-750) & Avg Temp $\left({ }^{\circ} \mathrm{C}\right)$ & Max Temp $\left({ }^{\circ} \mathrm{C}\right)$ \\
\hline EPRI-1B4 & $10 \mathrm{~A} 0002 \mathrm{~A} 07$ & $5.33 E+19$ & $8.64 \mathrm{E}-02$ & 339 & 405 \\
\hline EPRI-1B5 & 10A0002 A06 & $5.35 E+19$ & $8.68 \mathrm{E}-02$ & 342 & 405 \\
\hline EPRI-1B6 & $10 A 0002$ A05 & $5.33 E+19$ & $8.66 \mathrm{E}-02$ & 342 & 403 \\
\hline EPRI-1B7 & TEM Table & $5.35 E+19$ & $8.68 \mathrm{E}-02$ & 342 & 403 \\
\hline EPRI-1B8 & $10 \mathrm{~A} 0002 \mathrm{~A} 04$ & $5.35 E+19$ & $8.63 \mathrm{E}-02$ & 341 & 403 \\
\hline EPRI-1B9 & $10 A 0002$ A03 & $5.33 E+19$ & 8.63E-02 & 342 & 407 \\
\hline EPRI-1B10 & 10A0002 A02 & $5.35 E+19$ & $8.58 \mathrm{E}-02$ & 327 & 407 \\
\hline \multirow[t]{2}{*}{ EPRI-1B11 } & EP1-L3 (X750) & \multirow{2}{*}{$5.23 E+19$} & $8.23 \mathrm{E}-02$ & \multirow[t]{2}{*}{261} & \multirow[t]{2}{*}{279} \\
\hline & EP1-L4 (X750) & & $8.24 \mathrm{E}-02$ & & \\
\hline
\end{tabular}

Table 3-3: EPRI-1 XM-19 estimated irradiation temperature and fluence.

\begin{tabular}{|c|c|c|c|c|c|}
\hline Package & Specimen ID & Fluence $\left(\mathrm{n} / \mathrm{cm}^{2}\right)$ & dpa (XM-19) & Avg Temp $\left({ }^{\circ} \mathrm{C}\right)$ & Max Temp $\left({ }^{\circ} \mathrm{C}\right)$ \\
\hline EPRI-1B4 & 10A0001 A07 & $5.33 E+19$ & 7.95E-02 & 339 & 405 \\
\hline EPRI-1B5 & $10 A 0001 \mathrm{~A} 06$ & $5.35 E+19$ & $7.98 \mathrm{E}-02$ & 342 & 405 \\
\hline EPRI-1B6 & 10A0001 A05 & $5.33 E+19$ & 7.97E-02 & 342 & 403 \\
\hline EPRI-1B7 & TEM Table & $5.35 E+19$ & $7.98 \mathrm{E}-02$ & 342 & 403 \\
\hline EPRI-1B8 & 10A0001 A04 & $5.35 \mathrm{E}+19$ & 7.93E-02 & 341 & 403 \\
\hline EPRI-1B9 & $10 A 0001 \mathrm{~A} 03$ & $5.33 \mathrm{E}+19$ & 7.94E-02 & 342 & 407 \\
\hline EPRI-1B10 & $10 A 0001 \mathrm{~A} 02$ & $5.35 E+19$ & $7.89 \mathrm{E}-02$ & 327 & 407 \\
\hline \multirow[t]{2}{*}{ EPRI-1B11 } & XM-19 07 (XM19) & \multirow{2}{*}{$5.23 E+19$} & $7.56 \mathrm{E}-02$ & \multirow[t]{2}{*}{261} & \multirow[t]{2}{*}{279} \\
\hline & XM-19 02 (XM19) & & 7.57E-02 & & \\
\hline
\end{tabular}

\subsection{EPRI-2 Thermal and Neutronics}

The EPRI-2 experiment capsule was the very first irradiation conducted in the newly installed Loop 2A in the ATR CFT. It was irradiated in ATR cycle 153B, a so-called "Powered Axial Lift Mechanism" cycle which was started on March 31, 2013, and ran at full power for just over thirteen days, ending on April 13, 2013. The fast fluence target for this irradiation capsule was $2.0 \times 10^{20} \mathrm{n} / \mathrm{cm}^{2}$. Temperature was measured during the experiment utilizing packages of melt wires selected to span the target temperature of $288^{\circ} \mathrm{C}\left(550^{\circ} \mathrm{F}\right)$ from $239^{\circ} \mathrm{C}$ to $327^{\circ} \mathrm{C}$; this irradiation capsule was not outfitted with thermocouples like EPRI-1 and EPRI-3, which were subsequent to EPRI-2. Individual specimen irradiation temperatures were estimated in the post irradiation as-run analysis using an ABAQUS model that was compared to melt wire indications and calibrated to temperature change of coolant between inlet and outlet. An assumption of complete blockage of the space between specimens was made in order to bring estimates close to temperatures predicted by the melting of melt wires and based on observations noted earlier in this report. Reference [8] provides full details of the temperature prediction analysis. As with EPRI-1, an 
MCNP analysis was used to estimate the accumulated fluence for this irradiation. Reference [9] provides full details of the fluence calculation. Table 3-4 shows estimated fluence and minimum/maximum estimated irradiation temperatures for alloy X-750 specimens in this irradiation capsule. Table 3-5 shows estimated fluence and minimum/maximum estimated irradiation temperatures for XM-19 in this irradiation capsule. Yellow highlighted and blue highlighted cells identify specimens used for fracture toughness and IASCC testing in this project, respectively. Green highlighting indicates that the specimen was used for both types of test.

Table 3-4: EPRI-2 alloy X-750 estimated fluence and irradiation temperature.

\begin{tabular}{|c|c|c|c|c|c|}
\hline Package & Specimen ID & Fluence $\left(\mathrm{n} / \mathrm{cm}^{2}\right)$ & dpa (X-750) & $\operatorname{Min} \operatorname{Temp}\left({ }^{\circ} \mathrm{C}\right)$ & $\operatorname{Max} \operatorname{Temp}\left({ }^{\circ} \mathrm{C}\right)$ \\
\hline EPRI-2B4 & $10 \mathrm{~A} 0002 \mathrm{~B} 02$ & $1.94 \mathrm{E}+20$ & 0.310 & 349 & 359 \\
\hline EPRI-2B5 & $10 A 0002 \mathrm{~A} 12$ & $1.93 \mathrm{E}+20$ & 0.309 & 351 & 361 \\
\hline EPRI-2B6 & $10 A 0002 \mathrm{~A} 11$ & $1.92 \mathrm{E}+20$ & 0.307 & 351 & 361 \\
\hline EPRI-2B7 & TEM Table & $1.93 E+20$ & 0.309 & 351 & 360 \\
\hline EPRI-2B8 & $10 A 0002 \mathrm{~A} 10$ & $1.93 E+20$ & 0.309 & 349 & 359 \\
\hline EPRI-2B9 & 10A0002 A09 & $1.91 \mathrm{E}+20$ & 0.306 & 350 & 360 \\
\hline EPRI-2B10 & $10 A 0002 \mathrm{~A} 08$ & $1.89 \mathrm{E}+20$ & 0.302 & 329 & 338 \\
\hline \multirow[t]{2}{*}{ EPRI-2B11 } & EP3-L3 & \multirow{2}{*}{$1.83 E+20$} & 0.293 & \multirow[t]{2}{*}{230} & \multirow[t]{2}{*}{238} \\
\hline & EP3-L4 & & 0.293 & & \\
\hline
\end{tabular}

Table 3-5: EPRI-2 XM-19 estimated fluence and irradiation temperature.

\begin{tabular}{|c|c|c|c|c|c|}
\hline Package & Specimen ID & Fluence $\left(\mathrm{n} / \mathrm{cm}^{2}\right)$ & dpa (XM-19) & Min Temp $\left({ }^{\circ} \mathrm{C}\right)$ & $\operatorname{Max} \operatorname{Temp}\left({ }^{\circ} \mathrm{C}\right)$ \\
\hline EPRI-2B4 & 10A0001 B06 & $1.94 \mathrm{E}+20$ & 0.296 & 349 & 359 \\
\hline EPRI-2B5 & 10A0001 B05 & $1.93 \mathrm{E}+20$ & 0.294 & 351 & 361 \\
\hline EPRI-2B6 & 10A0001 B04 & $1.92 \mathrm{E}+20$ & 0.293 & 351 & 361 \\
\hline EPRI-2B7 & TEM Table & $1.93 \mathrm{E}+20$ & 0.294 & 351 & 360 \\
\hline EPRI-2B8 & 10A0001 B03 & $1.93 \mathrm{E}+20$ & 0.294 & 349 & 359 \\
\hline EPRI-2B9 & $10 \mathrm{~A} 0001 \mathrm{~B} 02$ & $1.91 \mathrm{E}+20$ & 0.291 & 350 & 360 \\
\hline EPRI-2B10 & 10A0001 B01 & $1.89 \mathrm{E}+20$ & 0.288 & 329 & 338 \\
\hline \multirow[t]{2}{*}{ EPRI-2B11 } & XM-19 03 (XM19) & \multirow{2}{*}{$1.83 \mathrm{E}+20$} & 0.279 & \multirow[t]{2}{*}{230} & \multirow[t]{2}{*}{238} \\
\hline & XM-19 04 (XM19) & & 0.279 & & \\
\hline
\end{tabular}

\subsection{EPRI-3 Thermal and Neutronics}

The EPRI-3 experiment capsule was irradiated over the course of two full ATR cycles: cycle 155B was initiated on February $13^{\text {th }}, 2014$, and ran for 58 days (50 effective days due to an 8-day reactor SCRAM) to April 12 ${ }^{\text {th }}, 2014$; and cycle 158 B was initiated on February 10, 2016, and ran for 51 days to April 1, 2016. This irradiation had a target fluence of $1.0 \times 10^{21} \mathrm{n} / \mathrm{cm}^{2}$. Similar to the EPRI-1 experiment, temperature was measured during this irradiation utilizing thermocouples embedded in specimen blanks near the top of the experiment and packages of melt wires selected to span the target temperature of 288 ${ }^{\circ} \mathrm{C}\left(550^{\circ} \mathrm{F}\right)$ from $239^{\circ} \mathrm{C}$ to $327^{\circ} \mathrm{C}$. Individual specimen irradiation temperatures were estimated in the post irradiation as-run analysis using an ABAQUS model that was compared to these thermocouple and 
melt wire indications. As discussed previously in Section 3.1.1, during cycle 155B (also referred to as EPRI 3-1) cruciform spacers were employed at the entry points of individual specimen holders in order to facilitate coolant flow through these areas (Figure 3-5); although this helped somewhat, the measured pressure drop during this cycle indicated that the flow restriction problem was not mitigated. Hence, full blockage of the flow channels between specimens was assumed in the model in order to match the temperatures estimated through the combination of melt wires and thermocouples. Average temperatures for some of the specimens are estimated to have reached as high as $347^{\circ} \mathrm{C}$ during this irradiation cycle. For the subsequent irradiation cycle, 158B (also referred to as EPRI 3-2), full flow restriction mitigation, including a flow through tube replacing the bottom dummy specimen stack and a centering mechanism (Figure 3-6 and Figure 3-7) was employed. Estimated average specimen temperatures during this cycle were significantly cooler. As with EPRI-1 and EPRI-2, an MCNP analysis was used to estimate the accumulated fluence for the EPRI-3 experiment over two cycles. Table 3-6 contains estimated fluence and irradiation temperatures for alloy X-750 in this irradiation capsule, and Table 3-7 contains estimated fluence and irradiation temperatures for XM-19 in this irradiation capsule. In Table 3-6 and Table 3-7, both average temperature and maximum temperatures are reported for cycles $155 \mathrm{~B}$ and $158 \mathrm{~B}$. Here, the average irradiation temperature is expected to be the temperature at which the specimens were held for a majority of the time while they would have been exposed to maximum temperature for brief time periods during power spikes. Yellow and blue highlighting identifies specimens used for fracture toughness and IASCC testing in this project, respectively. Full details of these as-run analyses may be found in references [10] and [11].

Table 3-6: EPRI-3 alloy X-750 estimated fluence and irradiation temperature.

\begin{tabular}{|c|c|c|c|c|c|}
\hline Package & Specimen ID & Fluence $\left(\mathrm{n} / \mathrm{cm}^{2}\right)$ & dpa (X-750) & $\begin{array}{c}\text { Avg Temp }\left({ }^{\circ} \mathrm{C}\right) \\
155 \mathrm{~B} / 158 \mathrm{~B} \\
\end{array}$ & $\begin{array}{c}\text { Max Temp }\left({ }^{\circ} \mathrm{C}\right) \\
155 \mathrm{~B} / 158 \mathrm{~B} \\
\end{array}$ \\
\hline EPRI-3B1 & $10 \mathrm{~A} 0002 \mathrm{CO3}$ & $9.395 \times 10^{20}$ & 1.490 & $301 / 264$ & $309 / 267$ \\
\hline EPRI-3B2 & $10 \mathrm{~A} 0002 \mathrm{CO}$ & $9.538 \times 10^{20}$ & 1.521 & $338 / 266$ & $351 / 269$ \\
\hline EPRI-3B3 & 10A0002 B12 & $9.538 \times 10^{20}$ & 1.527 & $333 / 266$ & $346 / 269$ \\
\hline EPRI-3B4 & 10A0002 B11 & $9.584 \times 10^{20}$ & 1.535 & $335 / 265$ & $347 / 268$ \\
\hline EPRI-3B5 & 10A0002 B10 & $9.629 \times 10^{20}$ & 1.538 & $335 / 265$ & $347 / 268$ \\
\hline EPRI-3B6 & $10 A 0002$ B09 & $9.704 \times 10^{20}$ & 1.542 & $335 / 265$ & $347 / 268$ \\
\hline EPRI-3B7 & TEM Table & $9.734 \times 10^{20}$ & 1.545 & $334 / 264$ & $347 / 267$ \\
\hline EPRI-3B8 & 10A0002 B08 & $9.610 \times 10^{20}$ & 1.538 & $333 / 264$ & $346 / 267$ \\
\hline EPRI-3B9 & 10A0002 B07 & $9.590 \times 10^{20}$ & 1.533 & $332 / 263$ & $345 / 266$ \\
\hline EPRI-3B10 & 10A0002 B06 & $9.532 \times 10^{20}$ & 1.524 & $331 / 263$ & $344 / 265$ \\
\hline EPRI-3B11 & 10A0002 B05 & $9.487 \times 10^{20}$ & 1.516 & $329 / 262$ & $341 / 265$ \\
\hline EPRI-3B12 & 10A0002 B04 & $9.346 \times 10^{20}$ & 1.493 & $330 / 261$ & $342 / 264$ \\
\hline EPRI-3B13 & $10 A 0002$ B03 & $8.658 \times 10^{20}$ & 1.441 & $287 / 257$ & $295 / 259$ \\
\hline \multirow{2}{*}{ EPRI-3A12 } & EP4-L3 & \multirow{2}{*}{$8.50 \times 10^{20}$} & \multirow{2}{*}{1.370} & \multirow{2}{*}{$255 / 260$} & \multirow{2}{*}{$258 / 263$} \\
\hline & EP4-L4 & & & & \\
\hline
\end{tabular}

Table 3-7: EPRI-3 XM-19 estimated fluence and irradiation temperature.

\begin{tabular}{|c|c|c|c|c|c|}
\hline Package & Specimen ID & Fluence $\left(\mathbf{n} / \mathbf{c m}^{2}\right)$ & dpa $(\mathrm{XM}-19)$ & $\begin{array}{c}\text { Avg Temp }\left({ }^{\circ} \mathrm{C}\right) \\
\mathbf{1 5 5 B} / \mathbf{1 5 8 B}\end{array}$ & $\begin{array}{c}\text { Max Temp }\left({ }^{\circ} \mathrm{C}\right) \\
155 \mathrm{~B} / \mathbf{1 5 8 B}\end{array}$ \\
\hline EPRI-3B1 & $10 \mathrm{A0001} \mathrm{E04}$ & $9.395 \times 10^{20}$ & 1.413 & $301 / 264$ & $309 / 267$ \\
\hline
\end{tabular}




\begin{tabular}{|c|c|c|c|c|c|}
\hline EPRI-3B2 & 10A0001 E03 & $9.538 \times 10^{20}$ & 1.448 & $338 / 266$ & $351 / 269$ \\
\hline EPRI-3B3 & 10A0001 E02 & $9.538 \times 10^{20}$ & 1.452 & $333 / 266$ & $346 / 269$ \\
\hline EPRI-3B4 & 10A0001 E01 & $9.584 \times 10^{20}$ & 1.459 & $335 / 265$ & $347 / 268$ \\
\hline EPRI-3B5 & $10 \mathrm{~A} 0001 \mathrm{D} 07$ & $9.629 \times 10^{20}$ & 1.462 & $335 / 265$ & $347 / 268$ \\
\hline EPRI-3B6 & 10A0001 D06 & $9.704 \times 10^{20}$ & 1.458 & $335 / 265$ & $347 / 268$ \\
\hline EPRI-3B7 & TEM Table & $9.734 \times 10^{20}$ & 1.460 & $334 / 264$ & $347 / 267$ \\
\hline EPRI-3B8 & 10A0001 D05 & $9.610 \times 10^{20}$ & 1.466 & $333 / 264$ & $346 / 267$ \\
\hline EPRI-3B9 & 10A0001 D04 & $9.590 \times 10^{20}$ & 1.461 & $332 / 263$ & $345 / 266$ \\
\hline EPRI-3B10 & 10A0001 D03 & $9.532 \times 10^{20}$ & 1.449 & $331 / 263$ & $344 / 265$ \\
\hline EPRI-3B11 & 10A0001 D02 & $9.487 \times 10^{20}$ & 1.443 & $329 / 262$ & $341 / 265$ \\
\hline EPRI-3B12 & 10A0001 D01 & $9.346 \times 10^{20}$ & 1.430 & $330 / 261$ & $342 / 264$ \\
\hline EPRI-3B13 & $10 \mathrm{~A} 0001 \mathrm{~B} 07$ & $8.658 \times 10^{20}$ & 1.320 & $287 / 257$ & $295 / 259$ \\
\hline \multirow{2}{*}{ EPRI-3A12 } & XM-19 01 & \multirow{2}{*}{$8.50 \times 10^{20}$} & \multirow{2}{*}{1.300} & \multirow{2}{*}{$255 / 260$} & \multirow{2}{*}{$258 / 263$} \\
\hline & XM-19 06 & & & & \\
\hline
\end{tabular}

\section{Post Irradiation Examination}

\subsection{Tensile Tests}

\subsubsection{Methodology}

Tensile tests for this project were completed using an Instron model 5800, $50 \mathrm{KN}$ capacity screwdriven test system located in the INL Hot Fuel Examination Facility and outfitted with a two-zone high temperature furnace (for EPRI-2 tests) or three-zone high temperature furnace (for EPRI-1 and EPRI-3 tests), and a modified Linear Variable Displacement Transducer (LVDT)-based extensometer (installed after EPRI-2 tests were completed). A typical test setup is shown in Figure 4-1. A schematic and photo of the LVDT extensometer used for EPRI-1 and EPRI-3 tests is shown in Figure 4-2. All tests were performed according to ASTM Standard E8, "Standard Test Methods for Tension Testing of Metallic Materials," [5] to the extent possible. The nominal gage cross-section was $6.35 \mathrm{~mm}$, and the effective gage length was $31.75 \mathrm{~mm}$ for all specimens. The specimen geometry is shown in Section 2.3.2 in this report. All specimens were tested in displacement control at a displacement rate of $0.276 \mathrm{~mm} / \mathrm{min}$ (strain rate $0.018 \%-\mathrm{s}^{-1}$ ) at a nominal temperature of $288^{\circ} \mathrm{C}$ within the gage section. Temperature was estimated based on an initial calibration procedure that involved measurement of the temperature in a surrogate tensile specimen outfitted with Type $\mathrm{K}$ thermocouples and heated to specific set points within the furnace zones. Force, extensometer, and crosshead displacement readings were recorded using Labview ${ }^{\circledR}$ software. For EPRI-2 tests, strain was measured using crosshead displacement, which was corrected for measured system compliance; EPRI-1 and EPRI-3 tests were conducted using the custom-made, LVDTbased extensometer which measures displacement directly on the gage section of the specimens. 


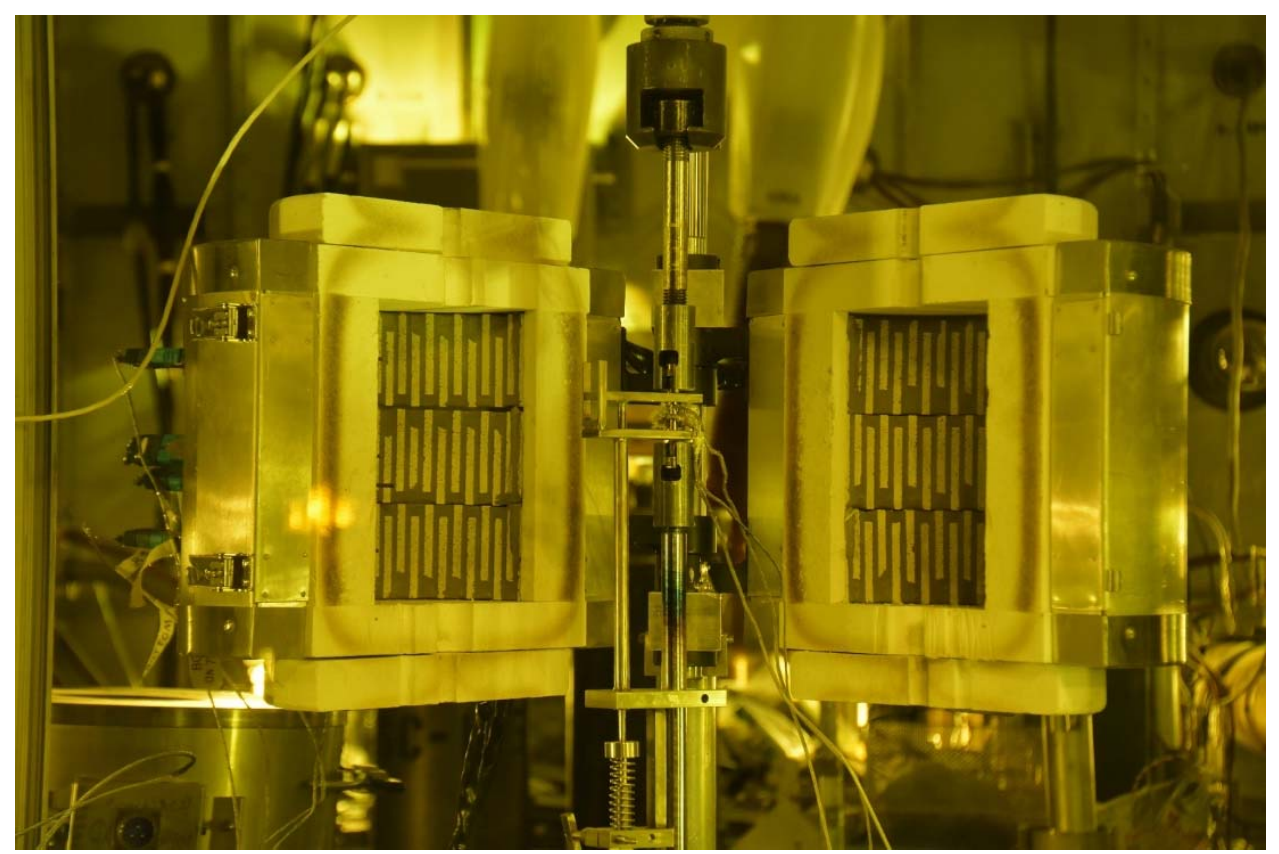

Figure 4-1: Typical tensile testing setup after installing a three-zone furnace and integration of an LVDT extensometer.
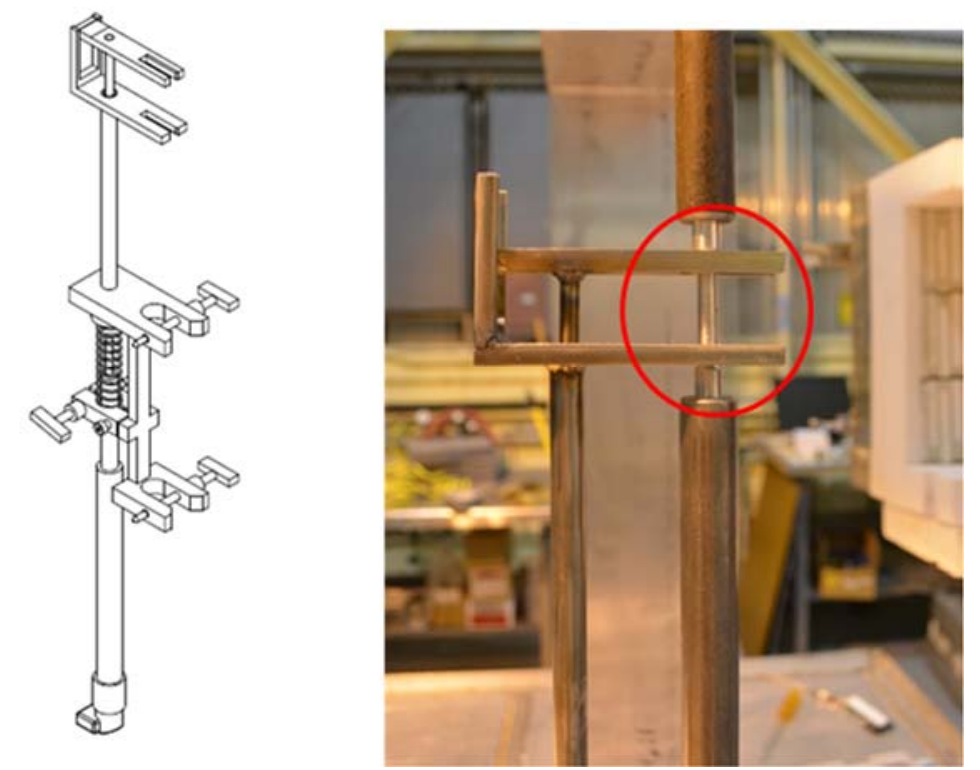

Figure 4-2: Schematic and photograph of LVDT strain measurement system.

\subsubsection{Alloy X-750 Tensile Results}

A total of six tensile tests were conducted at approximately $288^{\circ} \mathrm{C}$ on irradiated alloy X-750 for this project. Two tests were conducted for each fluence level. A $0.2 \%$ offset yield strength, ultimate tensile strength, and strain to failure were calculated using ASTM Standard E-8 [5] methodology in all cases. Table 4-1 shows the calculated values for each fluence along with, for comparison, values obtained from baseline tests [1]. 
Table 4-1: Irradiated and baseline tensile properties for alloy $\mathrm{X}-750$ at $288{ }^{\circ} \mathrm{C}$.

\begin{tabular}{|c|c|c|c|c|}
\hline Alloy X-750 Sample & Dose (dpa) & $\begin{array}{c}0.2 \% \text { Offset } \\
\text { Yield } \\
\text { (MPa) }\end{array}$ & $\begin{array}{c}\text { Ultimate Tensile } \\
\text { Strength } \\
\text { (MPa) }\end{array}$ & $\begin{array}{c}\text { Ductility } \\
\text { (\%) }\end{array}$ \\
\hline EP1-L5 (Ref. [1]) & 0 & 712 & 1076 & 32 \\
\hline EP1-L6 (Ref. [1]) & 0 & 715 & 1082 & 30 \\
\hline EP1-L3 $\left(5.23 \times 10^{19} \mathrm{n} / \mathrm{cm}^{2}\right)$ & 0.0823 & 867 & 1041 & 26 \\
\hline EP1-L4 $\left(5.23 \times 10^{19} \mathrm{n} / \mathrm{cm}^{2}\right)$ & 0.0824 & 865 & 1031 & 29 \\
\hline EP3-L3 $\left(1.83 \times 10^{20} \mathrm{n} / \mathrm{cm}^{2}\right)$ & 0.293 & 962 & 1067 & 13 \\
\hline EP3-L4 $\left(1.83 \times 10^{20} \mathrm{n} / \mathrm{cm}^{2}\right)$ & 0.293 & 877 & 1023 & 21 \\
\hline EP4-L3 $\left(0.86 \times 10^{21} \mathrm{n} / \mathrm{cm}^{2}\right)$ & 1.37 & 1129 & 1156 & 8 \\
\hline EP4-L4 $\left(0.86 \times 10^{21} \mathrm{n} / \mathrm{cm}^{2}\right)$ & 1.37 & 1113 & 1144 & 9 \\
\hline
\end{tabular}

As expected, yield strength increases with fluence, and ultimate strength also increase - albeit to a lesser extent than the yield strength, due to the nature of irradiation-induced hardening, which primarily affects the strain-hardening response due to the formation of dislocation loops. Accordingly, ductility is also reduced as a function of increasing fluence. Figure 4-3 is a plot of the yield strength, ultimate strength, and strain to failure as a function of dpa. Figure 4-4 is an overlay plot of alloy X-750 stressstrain curves for all fluence levels. 


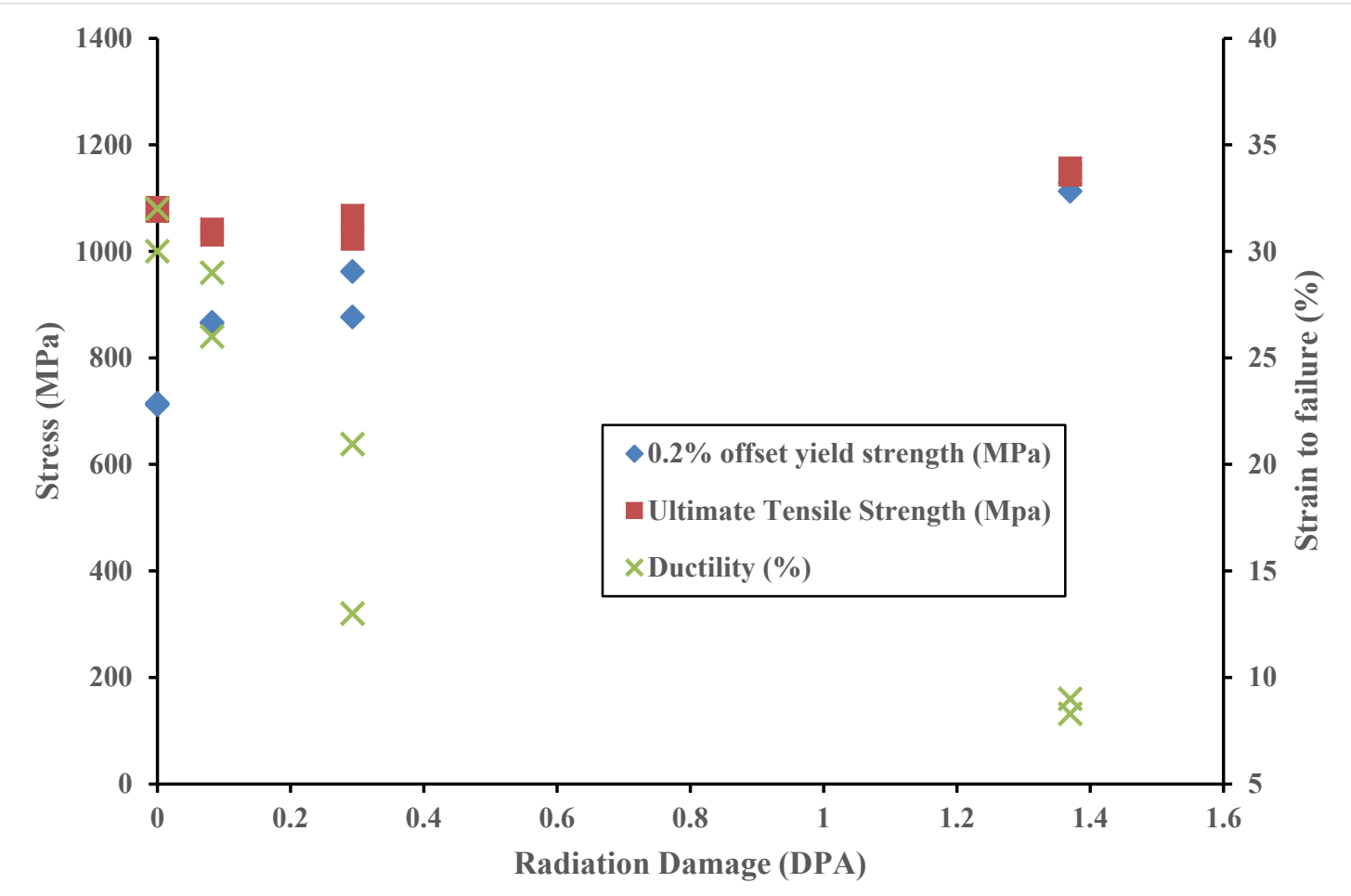

Figure 4-3: Trend of tensile properties at $288{ }^{\circ} \mathrm{C}$ as a function of dpa for alloy $\mathrm{X}-750$.

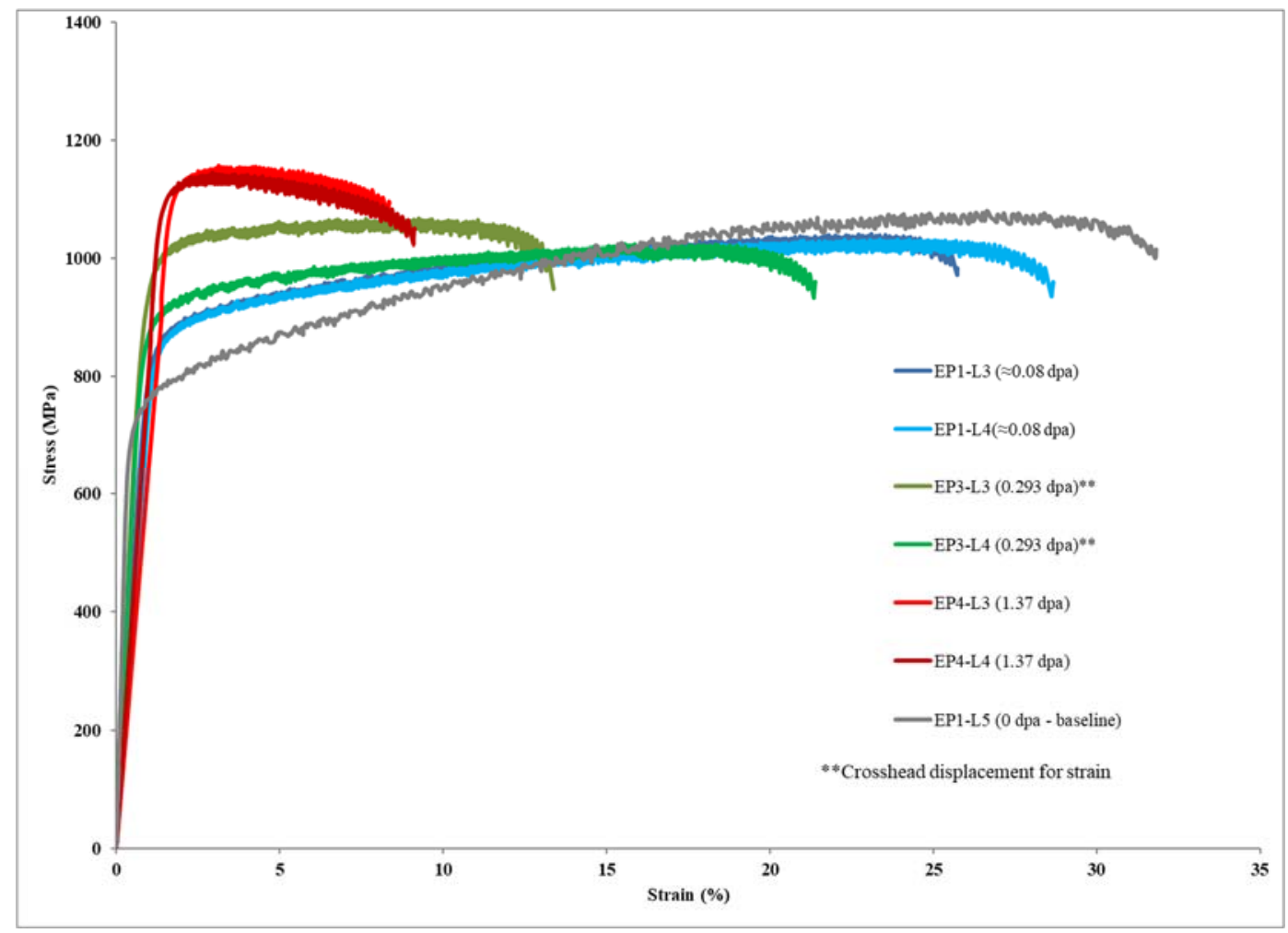

Figure 4-4: Overlay alloy X-750 stress-strain curves tested at $288^{\circ} \mathrm{C}$. 
It is noted that the two medium fluence alloy X-750 specimens (EP3-L3 and EP3-L4) show an unexpectedly large difference in yield properties, since they were irradiated in the same capsule within millimeters of each other. Therefore, a slice of the two samples was extracted out of the grip section of each specimen for microhardness testing. The Vickers hardness values appear to qualitatively support a difference in the tensile properties of the magnitude seen in the tensile tests. Additionally, the slides were etched, polished, and imaged under an optical microscope in order to observe the microstructure to see if anything could be deduced with regard to material differences. Figure 4-5 shows the Vickers hardness profile across the previously mentioned slices. Figure 4-6 shows the micrographs obtained after polishing and etching the specimens.

Although the microhardness values qualitatively support a possible difference in ultimate tensile strength, it is difficult to use these values to support the noted difference in yield strength. Correlations between microhardness and ultimate tensile strength exist in literature, but not for microhardness and yield strength. The micrographs in Figure 4-6 show a relatively consistent grain structure that is as expected based on material. Based on the microhardness values and microstructural observations, it is concluded that a testing issue, rather than a material issue, resulted in the difference in yield strength. The testing issue was most likely related to temperature control in the two zone furnace used for these initial tests.

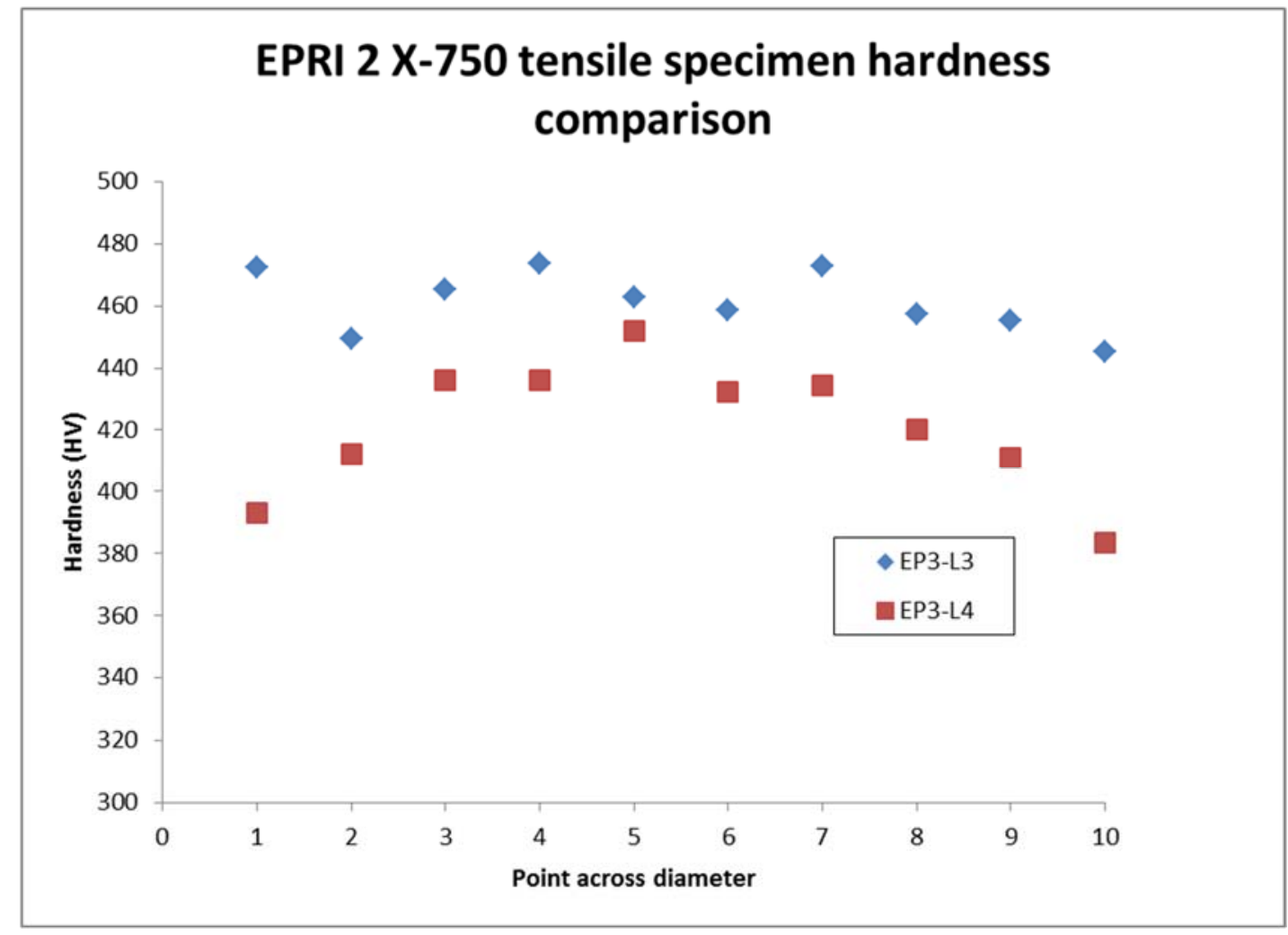

Figure 4-5: Vickers hardness measurements across the diameter of medium fluence alloy X-750 specimens. 


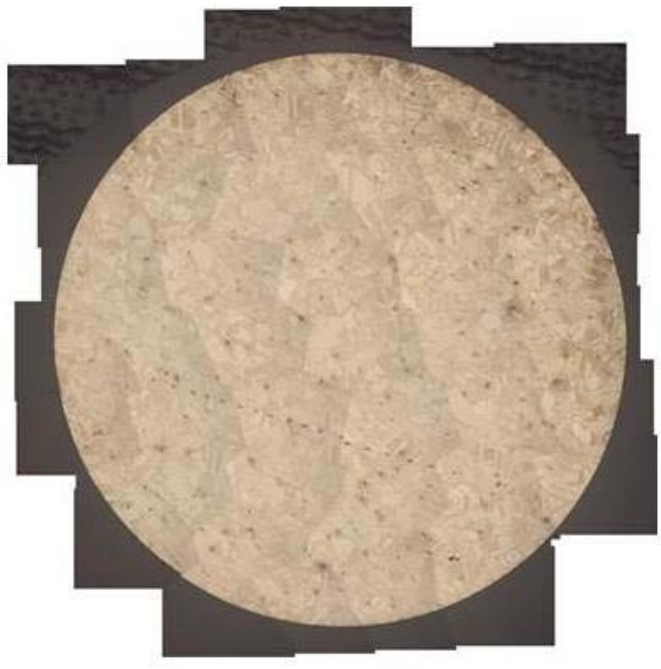

EP3-L3

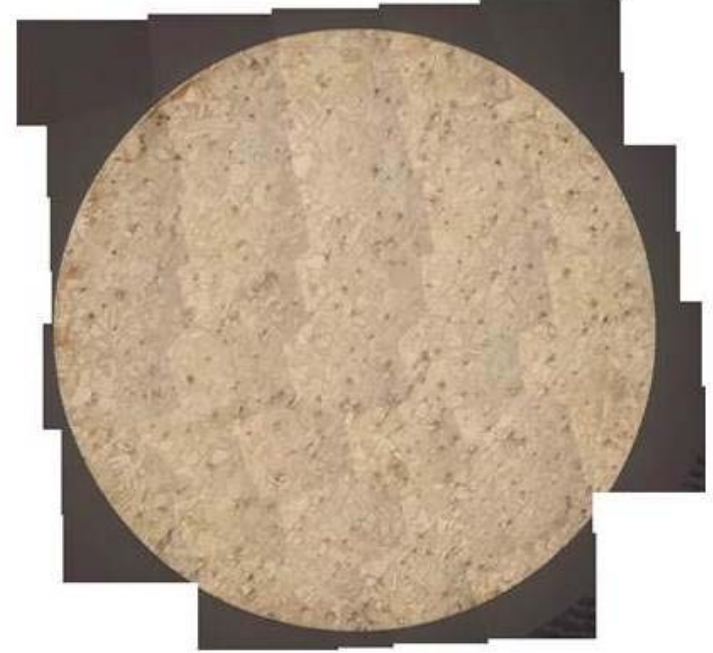

EP3-L4

\section{Figure 4-6: Micrographs showing consistent microstructure of medium fluence alloy X-750 specimens.}

\subsubsection{XM-19 Tensile Results}

A total of five tensile tests were successfully conducted on irradiated XM-19 for this project at a test temperature of nominally $288{ }^{\circ} \mathrm{C}$. Two tests were conducted for each fluence level, except for the medium fluence level, of which one of the two specimens was destroyed during test setup. A $0.2 \%$ offset yield strength, ultimate tensile strength, and strain to failure were calculated using ASTM Standard E-8 [5] methodology in all cases. Table 4-2 shows calculated values for each fluence along with, for comparison, values obtained from baseline tests [1]. Reference [12] is a vendor specification sheet that lists tensile properties for annealed XM-19 plate tested at $316^{\circ} \mathrm{C}$ and is the closest available comparison to a true baseline.

Similar to alloy X-750, yield strength for XM-19 increases with fluence, and ultimate strength also increases - albeit to a lesser extent than the yield strength, due to the nature of irradiation-induced hardening, which primarily affects the strain-hardening response due to the formation of dislocation loops. Accordingly, ductility is also reduced as a function of increasing fluence. In comparison to alloy X750, XM-19 exhibits a more significant increase in yield strength between baseline (unirradiated) results and $\sim 0.08 \mathrm{dpa}$; this is likely the result of the higher strain-hardening capacity that is affected by neutron embrittlement in stainless steel as compared to a nickel-based alloy. For this set of tests, a comparison of effects due to cold work (baseline results) with neutron embrittlement cannot be directly made, although the yield strength and ultimate strength of the 19.3\% cold-worked XM-19 (samples XM-19 CW 01 and XM-19 CW 03 tested in [1]) is beginning to approach the levels seen in the highest fluence (1.3 dpa) tests. It is assumed that a higher level of neutron exposure may produce a similar effect as that seen with $19.3 \%$ cold work. Figure 4-7 is a plot of the yield strength, ultimate strength, and strain to failure as a function of dpa for XM-19. Figure 4-8 is an overlay plot of XM-19 stress-strain curves for all fluence levels. 
Table 4-2: Irradiated and baseline tensile properties for XM-19 at $288^{\circ} \mathrm{C}$

\begin{tabular}{|c|c|c|c|c|}
\hline XM-19 Sample & Dose (dpa) & $\begin{array}{c}\text { 0.2\% Offset } \\
\text { Yield } \\
\text { (MPa) }\end{array}$ & $\begin{array}{c}\text { Ultimate Tensile } \\
\text { Strength } \\
\text { (MPa) }\end{array}$ & $\begin{array}{c}\text { Ductility } \\
\text { (\%) }\end{array}$ \\
\hline XM-19 CW 01 (Ref. [1]) & 0 & 678 & 786 & 14 \\
\hline XM-19 CW 03 (Ref. [1]) & 0 & 701 & 793 & 15 \\
\hline Ref. [12] - at 316 ${ }^{\circ} \mathrm{C}$ & 0 & 317 & 717 & 45 \\
\hline $\mathrm{XM}-1902\left(5.23 \times 10^{19} \mathrm{n} / \mathrm{cm}^{2}\right)$ & 0.0757 & 409 & 654 & 39 \\
\hline $\mathrm{XM}-1907\left(5.23 \times 10^{19} \mathrm{n} / \mathrm{cm}^{2}\right)$ & 0.0756 & 408 & 656 & 41 \\
\hline $\mathrm{XM}-1904\left(1.83 \times 10^{20} \mathrm{n} / \mathrm{cm}^{2}\right)$ & 0.279 & 468 & 686 & 32 \\
\hline $\mathrm{XM}-1903\left(1.83 \times 10^{20} \mathrm{n} / \mathrm{cm}^{2}\right)$ & 0.279 & & & 30 \\
\hline $\mathrm{XM}-1901\left(0.86 \times 10^{21} \mathrm{n} / \mathrm{cm}^{2}\right)$ & 1.3 & 611 & 738 & 31 \\
\hline $\mathrm{XM}-1906\left(0.86 \times 10^{21} \mathrm{n} / \mathrm{cm}^{2}\right)$ & 1.3 & 589 & 744 & 30 \\
\hline
\end{tabular}

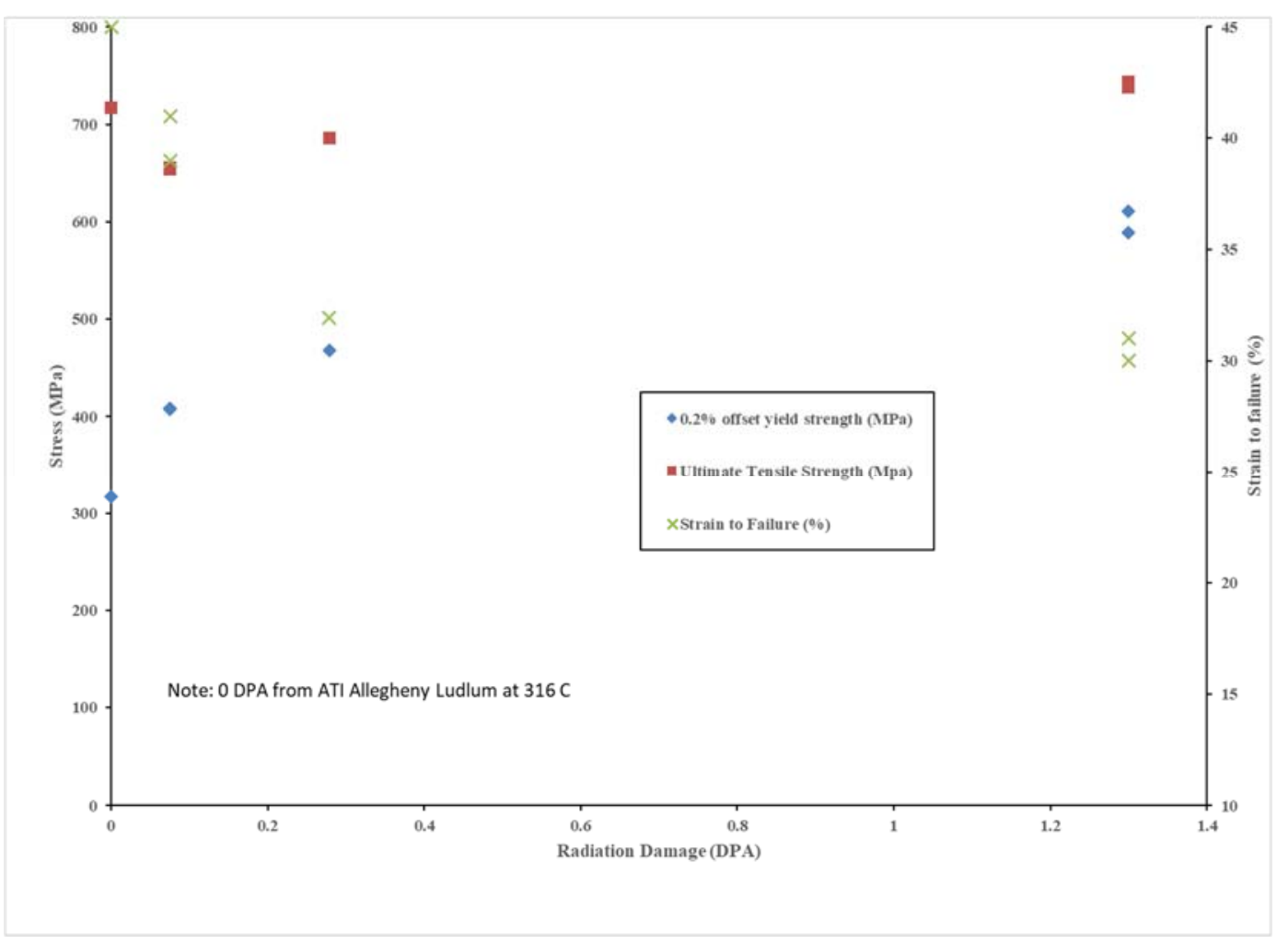

Figure 4-7: Trend of tensile properties as a function of dpa for XM-19 at $288{ }^{\circ} \mathrm{C}$. 


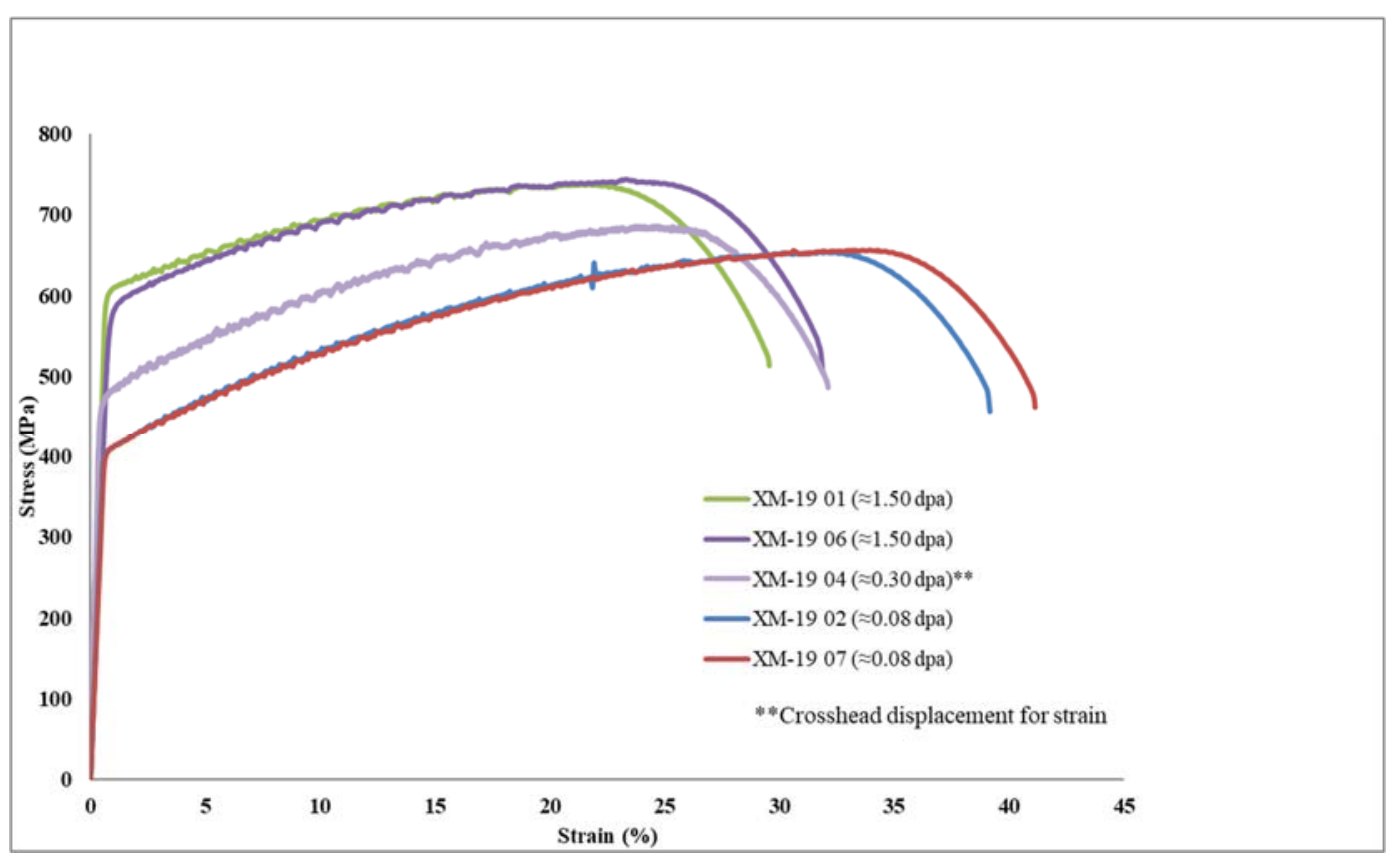

Figure 4-8: Overlay of irradiated XM-19 stress-strain curves at $288^{\circ} \mathrm{C}$.

\subsection{Fracture Toughness Tests}

Fracture toughness tests for this project were all performed using the IASCC test cells. All tests adhered to ASTM Standard E1820-09 [4] to the extent possible. The IASCC autoclaves were used to heat the fracture toughness specimens in air to approximately $288^{\circ} \mathrm{C}$ with temperature correlated to the internal Type $\mathrm{K}$ thermocouple located approximately $25 \mathrm{~mm}$ from the specimen. Since the autoclave was closed during the test and there was little space available, a crack mouth opening displacement gage was not utilized; rather, load line displacement corrected for measured system compliance was used for J-R calculations. Reversing direct current potential drop (DCPD) was utilized to measure crack extension during all of the tests. Crack length was estimated using this method based on an empirical model developed for current inputs located approximately $4 \mathrm{~mm}$ from the back face of the specimen and two sets of potential leads read diagonally across the mouth of the crack for redundancy (Figure 4-9). All DCPD crack length estimates were corrected to actual post-test measured crack length using a linear correction for calculation of the J-R curves. Electrical isolation of the specimens in these test systems was achieved using a double "clevis-in-clevis" design in which zirconia sleeves and washers are between the larger loading pin and the inner clevises attached to the specimens (Figure 4-10). 


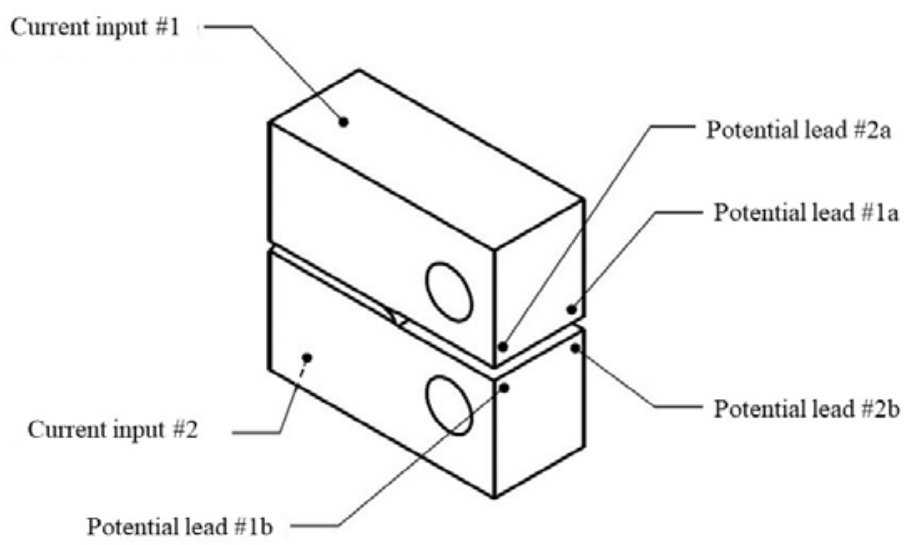

Figure 4-9: DCPD current inputs and potential leads.

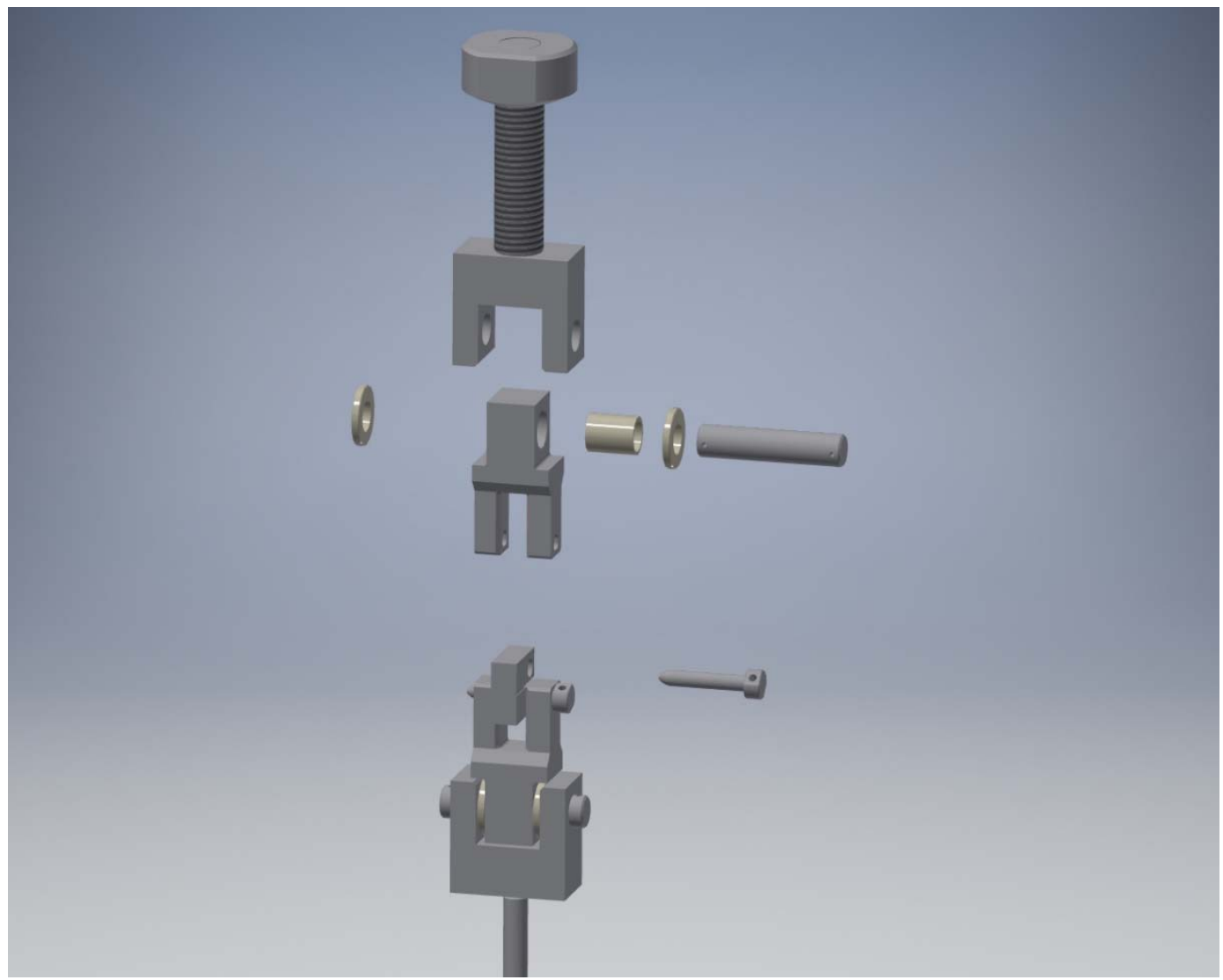

Figure 4-10: Exploded view of clevis-in-clevis specimen loading assembly showing zirconia insulating sleeves and washers. 


\subsubsection{Pre-fatigue and General Testing Details}

Specimens were pre-fatigued in air at testing temperature $\left(\sim 288^{\circ} \mathrm{C}\right)$ to a target crack length-to-width ratio of $a_{0} / \mathrm{W}=0.55-0.60$, as measured by DCPD. ASTM Standard E1820-09 [4] specifies an acceptable initial crack length-to-width ratio $\left(a_{0} / \mathrm{W}\right)$ of between 0.45 and 0.70 for a J-integral test. For this testing, the higher range is chosen in order to minimize the potential for loading pin damage due to high expected cracking forces in irradiated material. The maximum allowable applied stress intensity factor $(\mathrm{K})$ during the final step of fatigue pre-cracking, as per [4], is $\mathrm{K}_{\max }<0.6 \frac{\sigma_{f}}{\sigma_{T}} K_{F} \mathrm{MPaVm}$, where $\sigma_{\mathrm{f}}=$ yield strength at the fatigue temperature, $\sigma_{\mathrm{T}}$ is the yield strength at testing temperature, and $\mathrm{K}_{\mathrm{F}}$ is the measured fracture toughness, $\mathrm{K}_{\mathrm{Q}}$ or $\mathrm{K}_{\mathrm{C}}$ (qualified fracture toughness) for the material. Since tensile tests were all conducted at approximately $288^{\circ} \mathrm{C}$ for this project, $\sigma_{\mathrm{f}}$ is unknown and conservatively assumed to equal $85 \%$ of $\sigma_{\mathrm{T}}$. For alloy X-750 at the low, medium, and high fluence, the allowable $\mathrm{K}_{\max }$ is 84,76 , and $56 \mathrm{MPaVm}$, respectively, if the most conservative measurements of fracture toughness from the current project are used. For XM-19 at the low, medium, and high fluence, the allowable $\mathrm{K}_{\max }$ is 135,117 , and $109 \mathrm{MPaVm}$, respectively, if the most conservative measurements of fracture toughness from the current project are used. Applied $\mathrm{K}_{\max }$ in all cases is substantially lower than the allowable thresholds. It is noted that, in a single case for alloy XM-19 (specimen 10A0001B02), there was an IASCC test conducted prior to prefatiguing. Table 4-3 and Table 4-4 show pre-fatigue details for all of the alloy X-750 and XM-19 fracture toughness tests conducted in this project. All were pre-fatigued utilizing a sine wave loading pattern and utilizing load shedding to maintain constant $\mathrm{K}_{\max }$ as the pre-fatigue crack grew. It is noted that the final several specimens were pre-fatigued in multiple steps, simply due to the preference of the test operator; multiple steps are indicated by $-\mathrm{x}$ appended to the specimen name, where " $\mathrm{x}$ " is step number, and the final pre-fatigue crack length/width ratios are in bold in Table 4-3 and Table 4-4. There is no known effect of reducing frequency during fatigue pre-cracking except to increase the time necessary and reduce the risk of inadvertent specimen damage. Similarly, the ASTM standard [4] advises use of a load ratio equal to 0.1 , as it is the most aggressive; but for additional control, a less aggressive load ratio may be utilized, as was done in several cases.

Table 4-3: Pre-fatigue parameters for alloy $\mathrm{X}-750$ fracture toughness specimens.

\begin{tabular}{|c|c|c|c|c|}
\hline Specimen ID & $\begin{array}{c}\text { Applied } \mathbf{K}_{\mathbf{m a x}} \\
\left(\mathbf{M P a} \mathbf{m}_{\mathbf{m}}\right.\end{array}$ & $\begin{array}{c}\text { Load Ratio } \\
\left(\mathbf{K}_{\mathbf{m i n}} / \mathbf{K}_{\mathbf{m a x}}\right)\end{array}$ & $\begin{array}{c}\text { Frequency } \\
(\mathbf{h z})\end{array}$ & $\begin{array}{c}\text { Final } \\
\mathbf{a} / \mathbf{W}\end{array}$ \\
\hline 10A0002A09 & 40 & 0.1 & 1 & $\mathbf{0 . 5 8 0 5 2}$ \\
\hline 10A0002A11 & 25 & 0.2 & 1 & $\mathbf{0 . 5 5 0 0 9}$ \\
\hline 10A0002B08 & 25 & 0.2 & 1 & $\mathbf{0 . 5 5 0 0 2}$ \\
\hline 10A0002B10 & 25 & 0.2 & 0.5 & $\mathbf{0 . 5 6 3 3 9}$ \\
\hline 10A0002C03-1 & 30 & 0.1 & 0.5 & 0.48005 \\
\hline 10A0002C03-2 & 25 & 0.1 & 0.5 & $\mathbf{0 . 5 5 0 0 4}$ \\
\hline 10A0002C03-3 & 25 & 0.3 & 0.5 & 0.40987 \\
\hline 10A0002A07-1 & 30 & 0.1 & 0.5 & 0.4801 \\
\hline 10A0002A07-2 & 35 & 0.1 & 0.5 & 0.52008 \\
\hline 10A0002A07-3 & 30 & 0.3 & 0.5 & $\mathbf{0 . 5 5 0 0 3}$ \\
\hline 10A0002A07-4 & 30 & 0.1 & 0.5 & 0.40052 \\
\hline 10A0002A02-1 & 40 & 0.1 & 0.5 & 0.43101 \\
\hline 10A0002A02-2 & 35 & 0.1 & 0.5 & 0.52023 \\
\hline 10A0002A02-3 & 25 & 0.2 & & \\
\hline 10A0002A02-4 & 25 & & 0.5 & 0.5 \\
\hline
\end{tabular}




\begin{tabular}{|l|l|l|l|l|}
$10 \mathrm{~A} 0002 \mathrm{~A} 02-5$ & 25 & 0.3 & 0.5 & $\mathbf{0 . 5 5 0 1 1}$ \\
\hline
\end{tabular}

Table 4-4: Pre-fatigue parameters for XM-19 fracture toughness specimens.

\begin{tabular}{|c|c|c|c|c|}
\hline Specimen ID & $\begin{array}{c}\text { Applied } \mathbf{K}_{\text {max }} \\
\left(\mathbf{M P a}{ }_{\mathbf{m}}\right)\end{array}$ & $\begin{array}{c}\text { Load Ratio } \\
\left(\mathbf{K}_{\mathbf{m i n}} / \mathbf{K}_{\mathbf{m a x}}\right)\end{array}$ & $\begin{array}{c}\text { Frequency } \\
(\mathbf{h z})\end{array}$ & $\begin{array}{c}\text { Final } \\
\mathbf{a} / \mathbf{W}\end{array}$ \\
\hline 10A0001B01 & 25 & 0.1 & 1 & $\mathbf{0 . 5 2 5 0 3}$ \\
\hline 10A0001B02-1 & 25 & IASCC test & N/A & 0.46782 \\
\hline 10A0001B02-2 & 25 & 0.3 & 1 & $\mathbf{0 . 6 0 3 3 6}$ \\
\hline 10A0001D05-1 & 25 & 0.2 & 1 & 0.4113 \\
\hline 10A0001D05-2 & 25 & 0.1 & 1 & 0.41137 \\
\hline 10A0001D05-3 & 30 & 0.1 & 1 & 0.41364 \\
\hline 10A0001D05-4 & 29 & 0.1 & 2 & $\mathbf{0 . 5 5 0 0 8}$ \\
\hline 10A0001D05-5 & 28 & 0.1 & 0.5 & 0.48 \\
\hline 10A0001D01-1 & 30 & 0.1 & 0.5 & 0.52002 \\
\hline 10A0001D01-2 & 25 & 0.1 & 0.5 & $\mathbf{0 . 5 5 0 0 1}$ \\
\hline 10A0001D01-3 & 25 & 0.3 & 0.5 & 0.48022 \\
\hline 10A0001E04-1 & 30 & 0.1 & 0.5 & 0.52005 \\
\hline 10A0001E04-2 & 25 & 0.1 & 0.5 & $\mathbf{0 . 5 5 0 0 8}$ \\
\hline 10A0001E04-3 & 25 & 0.3 & 0.5 & 0.43492 \\
\hline 10A0001A07-1 & 30 & 0.1 & 0.5 & 0.48091 \\
\hline 10A0001A07-2 & 35 & 0.1 & 0.5 & 0.52019 \\
\hline 10A0001A07-3 & 25 & 0.1 & 0.5 & $\mathbf{0 . 5 5 0 2}$ \\
\hline 10A0001A07-4 & 25 & 0.3 & 0.5 & 0.48043 \\
\hline 10A0001A02-1 & 30 & 0.1 & 0.5 & 0.52006 \\
\hline 10A0001A02-2 & 25 & 0.1 & $\mathbf{0 . 5 5 0 3 2}$ \\
\hline 10A0001A02-3 & 25 & 0.3 & & \\
\hline
\end{tabular}

\subsubsection{Alloy X-750 Fracture Toughness Tests}

\subsubsection{Lowest fluence alloy $X$-750 fracture toughness}

Two fracture toughness tests were conducted for the lowest fluence alloy X-750 (EPRI-1). Specimens 10A0002A02 and 10A0002A07 both accumulated approximately 0.086 dpa of irradiation damage. Irradiation temperature for specimen $10 \mathrm{~A} 0002 \mathrm{~A} 02$ was estimated to be $327^{\circ} \mathrm{C}$ on average, with a maximum of $407^{\circ} \mathrm{C}$ during temporary reactor power spikes. Irradiation temperature for 10A0002A07 was estimated to be approximately $339^{\circ} \mathrm{C}$ on average, with a maximum of $405{ }^{\circ} \mathrm{C}$. Both specimens were monotonically loaded at a displacement rate equal to $1.0 \times 10^{-4} \mathrm{~mm} / \mathrm{s}$ for the initial portion of the test up to the maximum applied force, then the displacement rate was increased to $1.0 \times 10^{-3} \mathrm{~mm} / \mathrm{s}$ to finish the test. Post-fatigue marking was conducted at a $\mathrm{K}_{\max }$ equal to less than $50 \%$ of the final applied $\mathrm{K}$.

Both low fluence specimens exhibited ductile fracture morphology consisting of dimple rupture networks indicative of micro-void coalescence, along with the telltale necking of the specimen. Neither 
test allows qualification of $\mathrm{J}_{\mathrm{Q}}$ as $\mathrm{J}_{\mathrm{IC}}$ according to [4], since they violate minimum allowable crack tunneling requirements and, in the case of specimen $10 \mathrm{~A} 0002 \mathrm{~A} 07$, the crack extension consistency requirement is also violated. Of the two tests, $10 \mathrm{~A} 0002 \mathrm{~A} 02$ is considered to be the more viable result, as it nearly meets the requirements. Specimen 10A0002A07 was likely misaligned in the loading fixture, leading to the grossly non-uniform pre-crack as well as final crack front. Compared to baseline (unirradiated) results from [1], fracture toughness is actually increased from approximately $160 \mathrm{~kJ} / \mathrm{m}^{2}$ to approximately $180 \mathrm{~kJ} / \mathrm{m}^{2}$, as noted above. As suggested, this may be an artifact due to the negligible effect of irradiation on fracture toughness at this low level, combined with a lower constraint in the 0.4TCT specimen compared to the 0.5T-CT specimen utilized in baseline tests. Figure 4-11 and Figure 4-12 show the J-R curves and fracture surfaces for these low fluence tests.

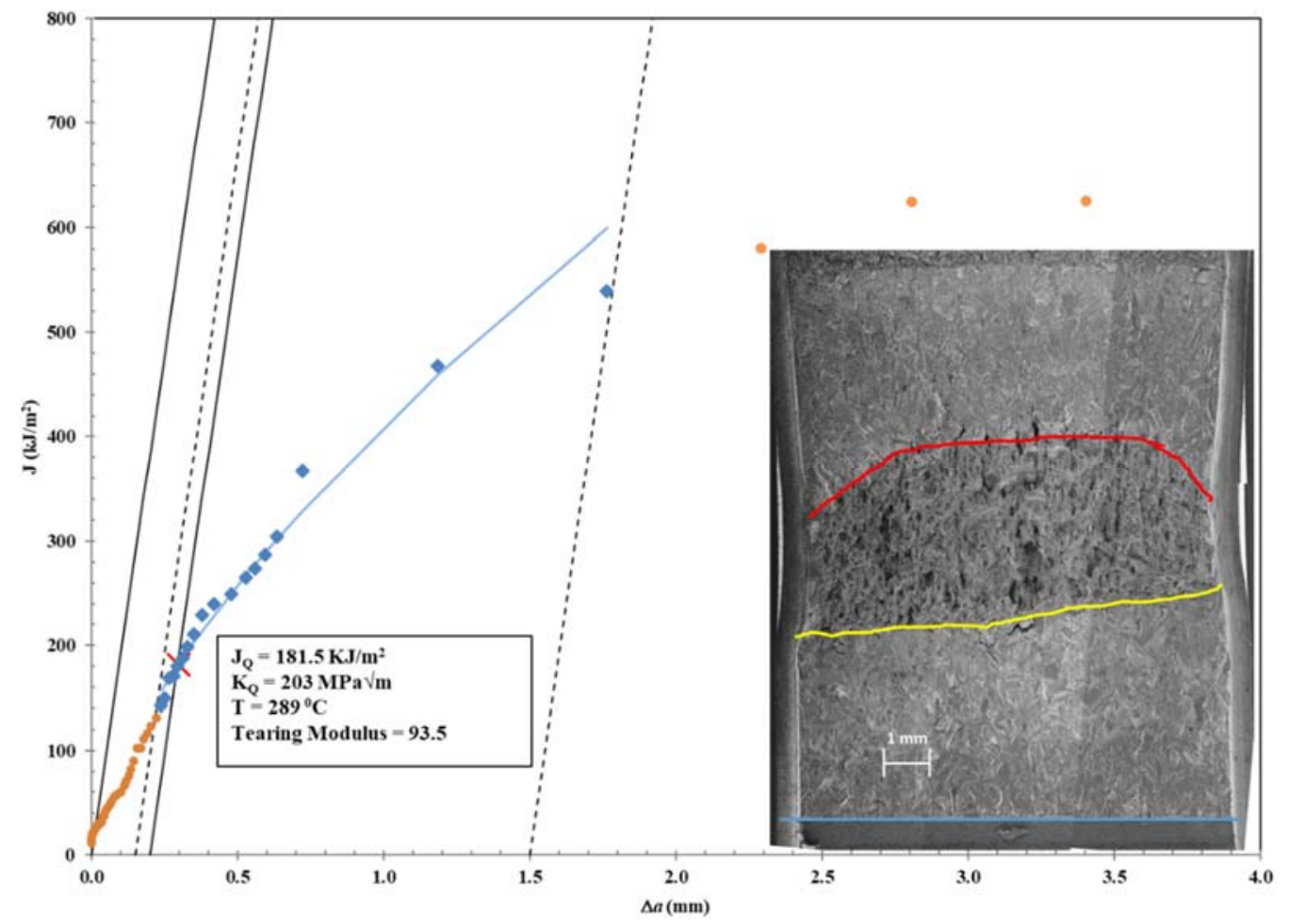

Figure 4-11: J-R curve and fracture surface for 10A0002A02 (alloy X-750, 0.086 dpa) specimen. 


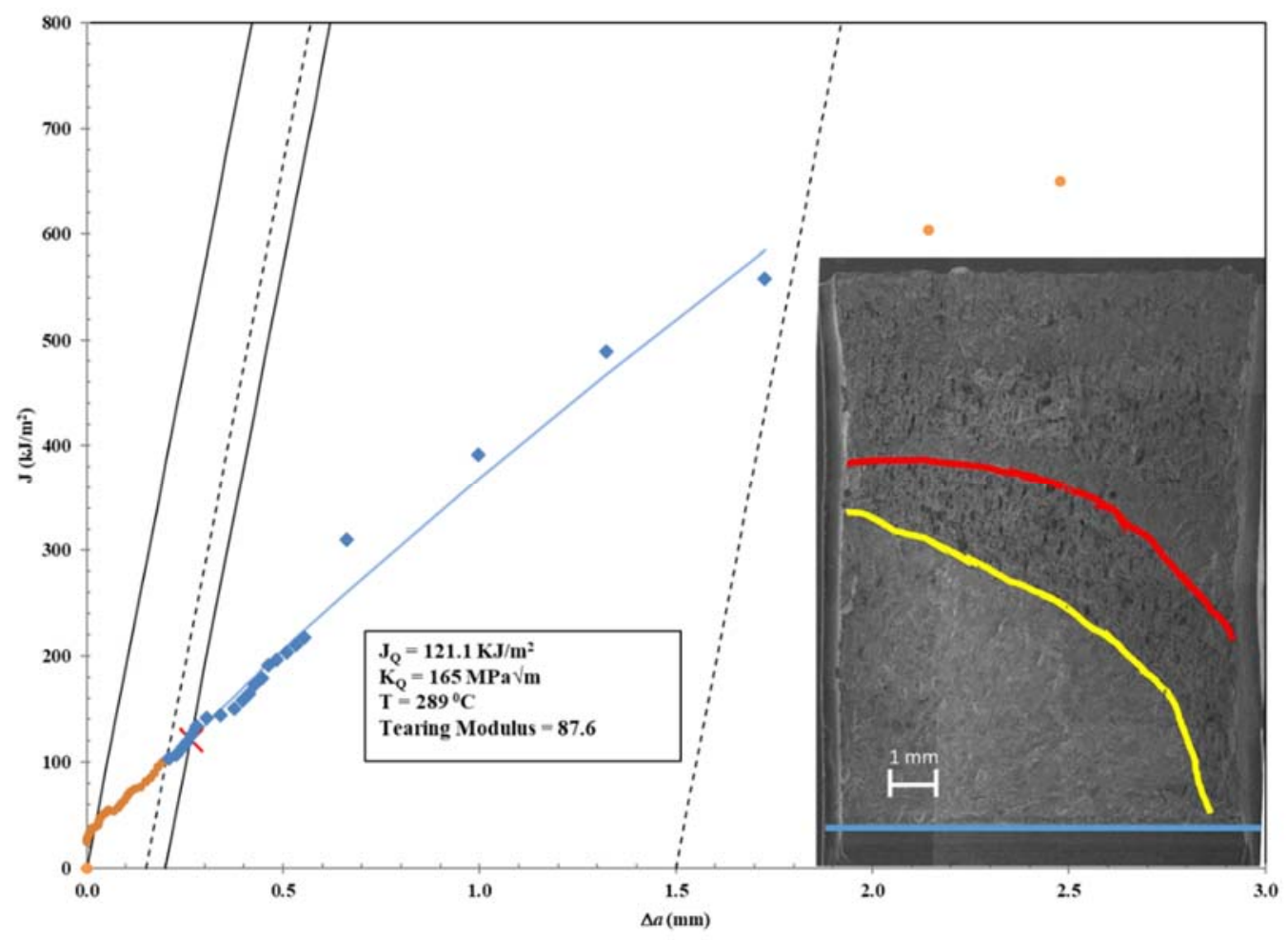

Figure 4-12: J-R curve and fracture surface for 10A0002A07 (alloy X-750, $0.086 \mathrm{dpa}$ ) specimen.

\subsubsection{Medium fluence alloy $X-750$ fracture toughness}

Two fracture toughness tests were conducted for the medium fluence alloy X-750 (EPRI-2). Specimens 10A0002A09 and 10A0002A11 both accumulated approximately 0.306 dpa of irradiation damage. Irradiation temperature for specimen $10 \mathrm{~A} 0002 \mathrm{~A} 09$ was estimated to be $350-360{ }^{\circ} \mathrm{C}$. Irradiation temperature for $10 \mathrm{~A} 0002 \mathrm{~A} 11$ was estimated to be approximately $351-361{ }^{\circ} \mathrm{C}$. Both specimens were monotonically loaded at a displacement rate equal to $4 \times 10^{-4} \mathrm{~mm} / \mathrm{s}$ for the duration of the test; this was based on previous loading rates used in [1]. These two tests were the very first fracture toughness tests performed in the newly installed IASCC test cells, so the methodology differs slightly from subsequent tests, as system response was previously uncharacterized. Post-fatigue marking was conducted at a $\mathrm{K}_{\max }$ equal to less than $50 \%$ of the final applied $\mathrm{K}$.

Both medium fluence specimens exhibited ductile fracture morphology consisting of dimple rupture networks indicative of micro-void coalescence, along with the telltale necking of the specimen. Neither test allows qualification of $\mathrm{J}_{\mathrm{Q}}$ as $\mathrm{J}_{\mathrm{IC}}$ according to [4], since they violate minimum allowable crack tunneling requirements. Both specimens exhibit extensive crack tunneling, suggesting a high stress triaxiality at the center of the crack tip as commonly seen in ductile materials with insufficient thickness. Figure 4-13 and Figure 4-14 show J-R curves and fracture surfaces for specimens 10A0002A09 and 10A0002A11. 


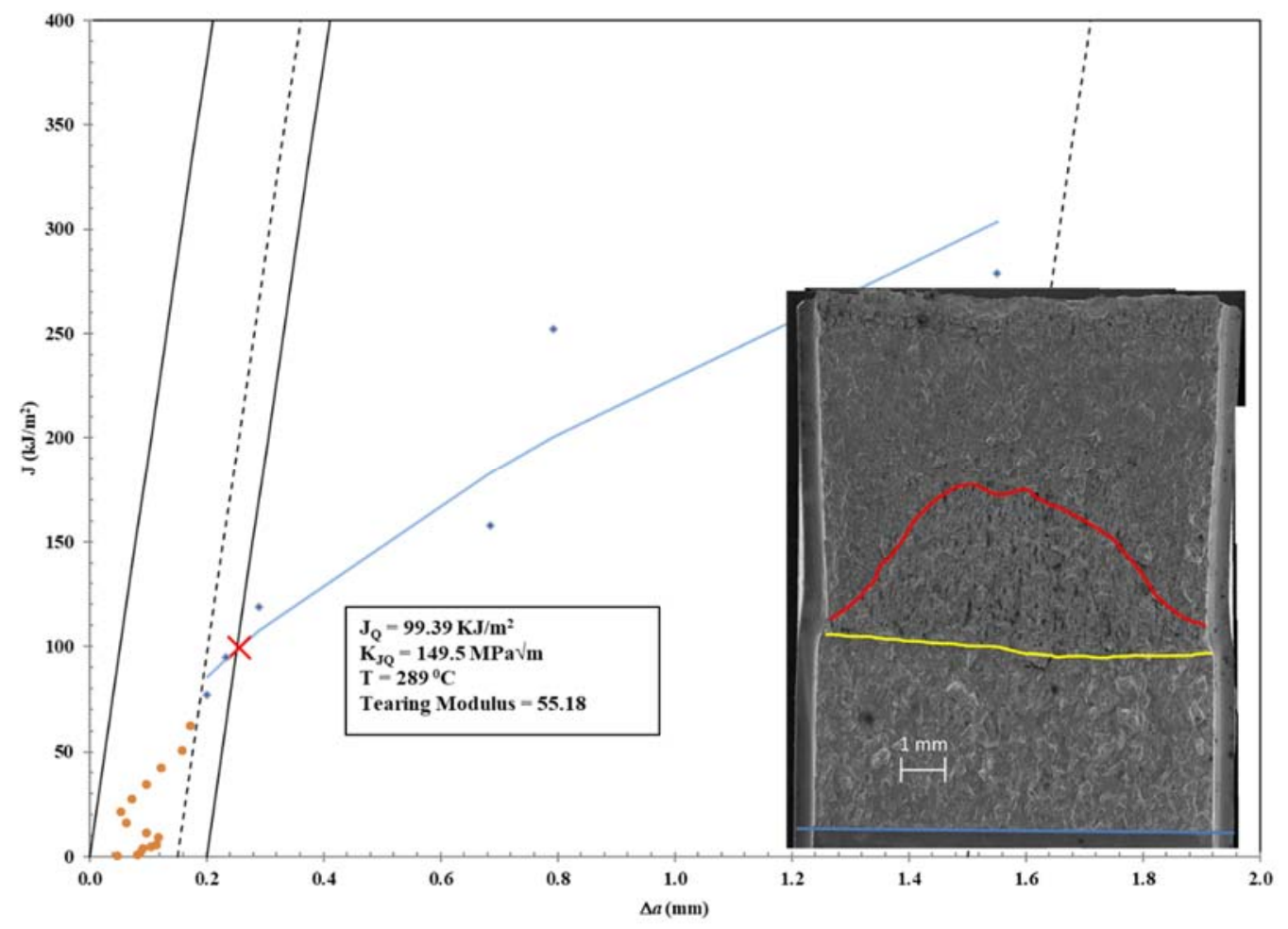

Figure 4-13: J-R curve and fracture surface for 10A0002A09 (alloy X-750, $0.306 \mathrm{dpa}$ ) specimen.

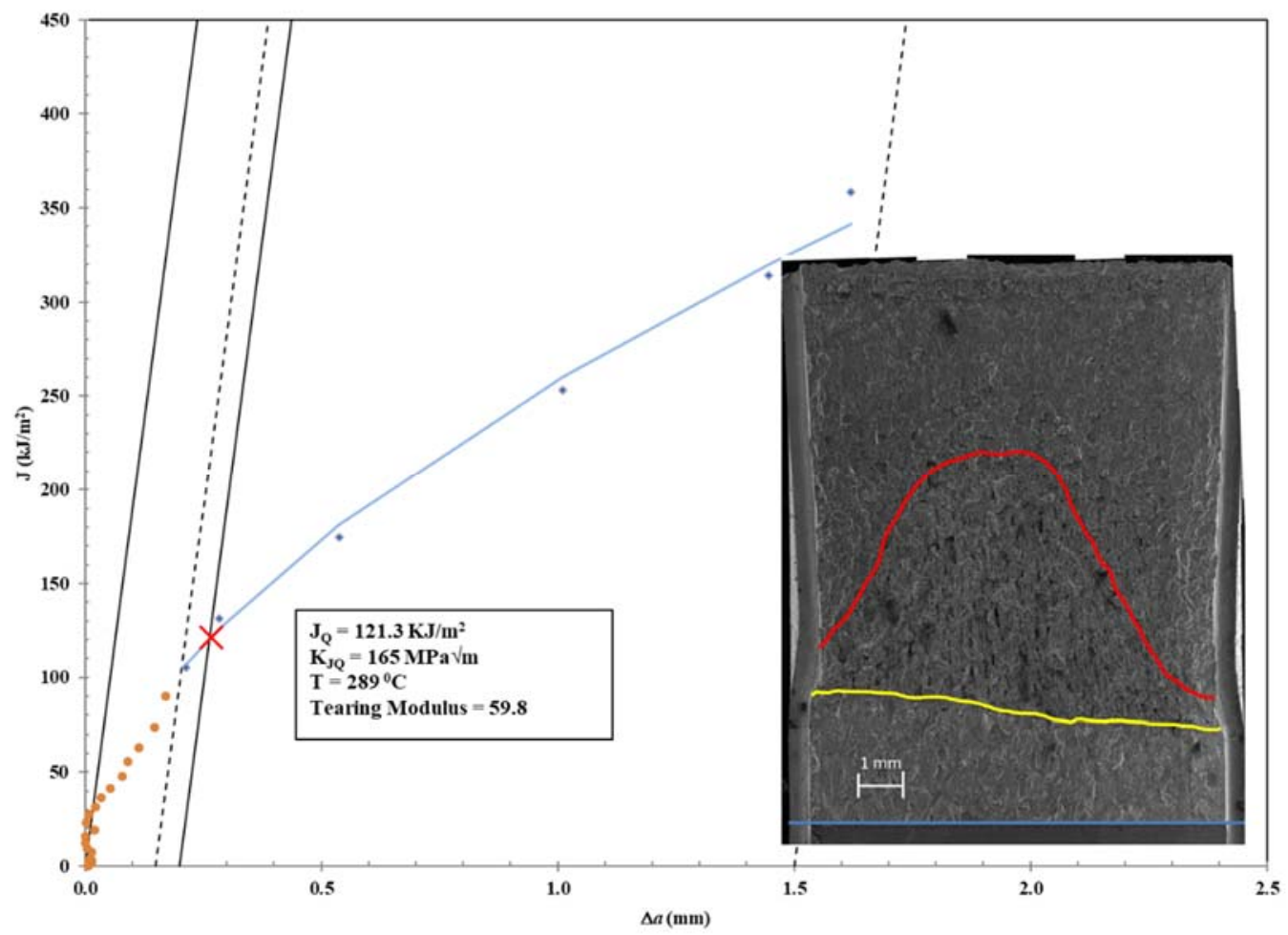

Figure 4-14: J-R curve and fracture surface for 10A0002A11 (alloy X-750, 0.307 dpa) specimen. 


\subsubsection{Highest fluence alloy $X-750$ fracture toughness}

Three fracture toughness tests were conducted for the highest fluence alloy X-750 (EPRI-3). Specimens 10A0002B08, 10A0002B10, and 10A0002C03 accumulated estimated irradiation damage levels of 1.54, 1.54, and 1.49 DPA, respectively. Specimens 10A0002B08 and 10A0002B10's average irradiation temperatures were estimated to be approximately $334{ }^{\circ} \mathrm{C}$ during the first irradiation cycle, and approximately $265^{\circ} \mathrm{C}$ during the second irradiation cycle, with maximum short-duration temperatures reaching as high as $347^{\circ} \mathrm{C}$ and $268^{\circ} \mathrm{C}$ during the first and second irradiation cycles, respectively. Specimen 10A0002C03, which was positioned closer to the loop coolant inlet, is estimated to have been irradiated at slightly cooler average temperatures of approximately $301{ }^{\circ} \mathrm{C}$ and $264{ }^{\circ} \mathrm{C}$ during the first and second irradiation cycles, with estimated short-duration maximums of approximately $309^{\circ} \mathrm{C}$ and $267^{\circ} \mathrm{C}$ during the first and second irradiation cycles, respectively.

Specimens 10A0002B08 and 10A0002B10 were monotonically loaded at a displacement rate of 1.67 $\times 10^{-3} \mathrm{~mm} / \mathrm{s}$ and a $17 \mathrm{X}$ slower rate of $1.0 \times 10^{-4} \mathrm{~mm} / \mathrm{s}$ for $10 \mathrm{~A} 0002 \mathrm{C} 03$ to ensure no loading rate effect. In all three fracture toughness tests for the highest fluence alloy X-750, it is clear that a significant reduction in ductility had occurred, consistent with the tensile testing results that show complete loss of capacity for strain hardening. All three fracture toughness tests exhibited linear elastic behavior with only slight nonlinearity before rapid fracture. Linear elastic fracture toughness was estimated using the $95 \%$ secant method outlined in [4], resulting in qualified $\left(\mathrm{K}_{\mathrm{IC}}\right)$ values of 112, 115, and $110 \mathrm{MPaVm}$ for specimens 10A0002B08, 10A0002B10, and 10A0002C03, respectively. Figure 4-15, Figure 4-16, and Figure 4-17 show force-displacement (in blue) curves for the three specimens with overlaid $95 \%$ secant lines; because of the rapid fracture associated with these tests, J-R curves are irrelevant. A closeup of the fracture surface for specimen 10A0002C03 is shown in Figure 4-18, with cleavage fracture being predominant, as expected based on the linear nature of the fracture toughness test. There are no apparent effects from the different irradiation temperatures. It is noted that, while an obvious change in the material's fracture characteristics has occurred at this irradiation damage level, the residual fracture toughness is still considered quite high.
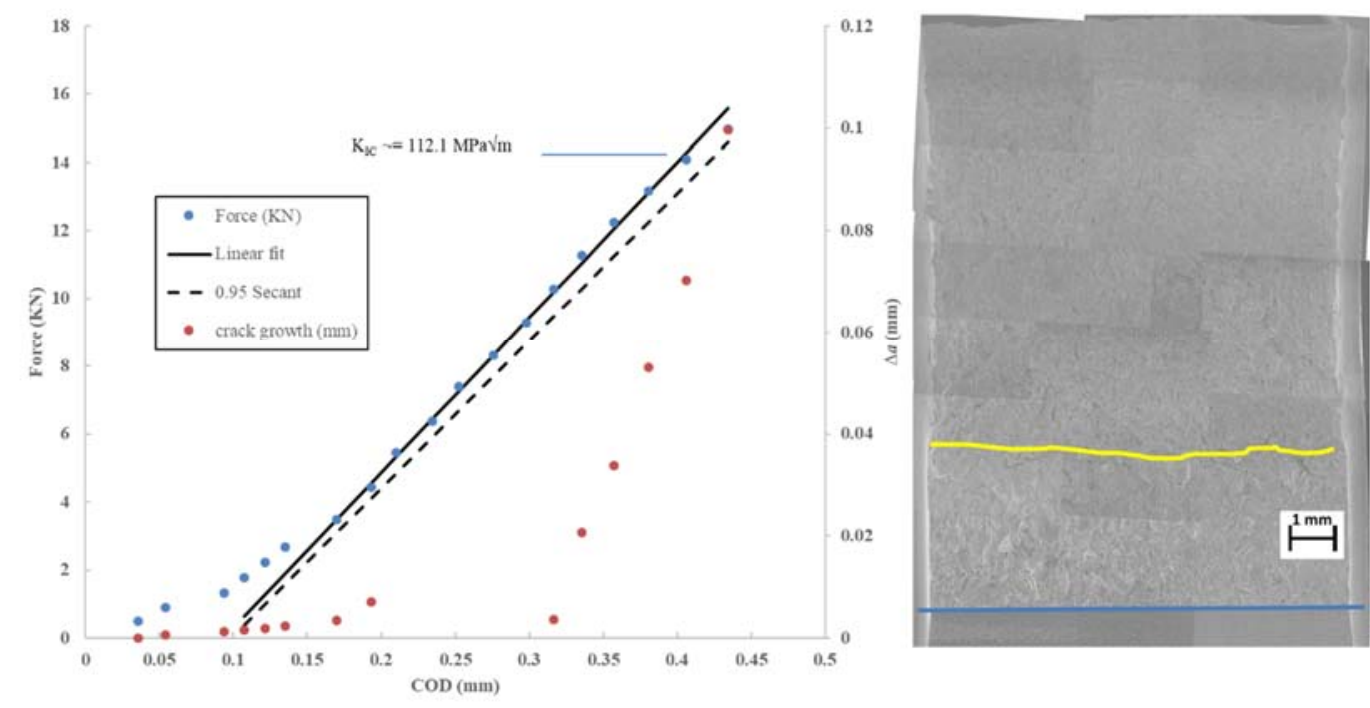

Figure 4-15: Force, displacement, crack growth, and fracture surface for 10A0002B08 (alloy X-750, 1.54 dpa) specimen. 

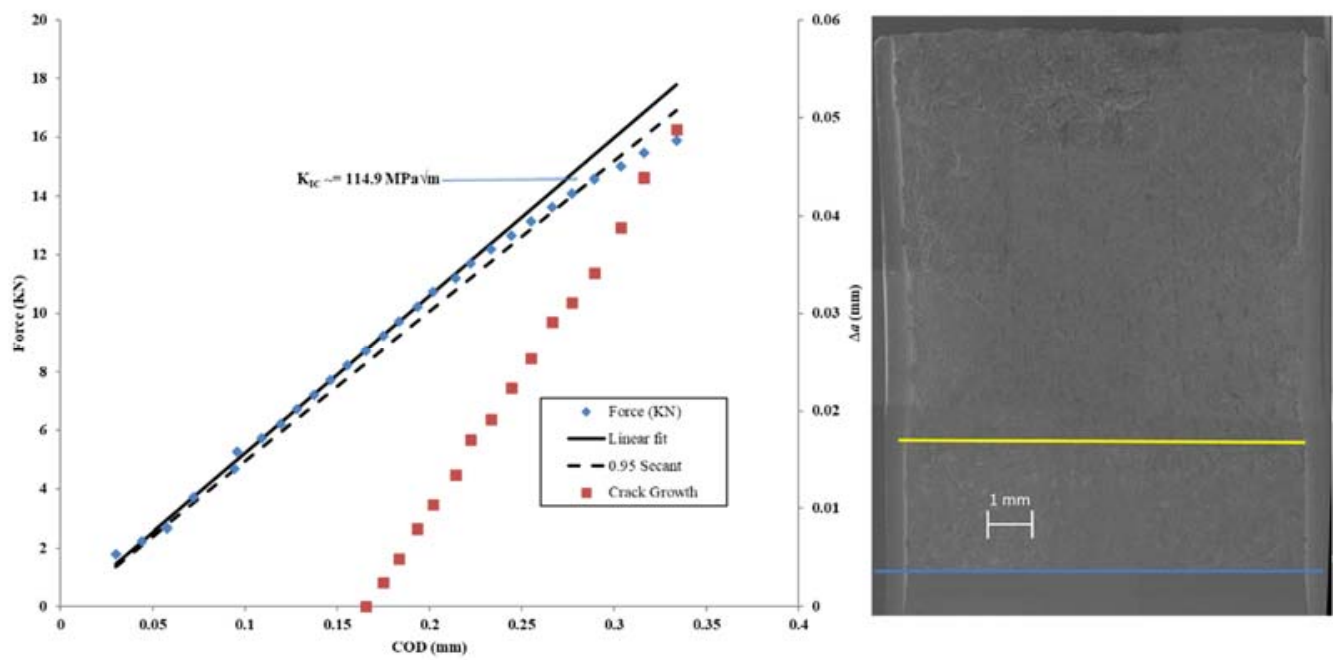

Figure 4-16: Force, displacement, crack growth, and fracture surface for 10A0002B10 (alloy X-750, 1.54 dpa) specimen.
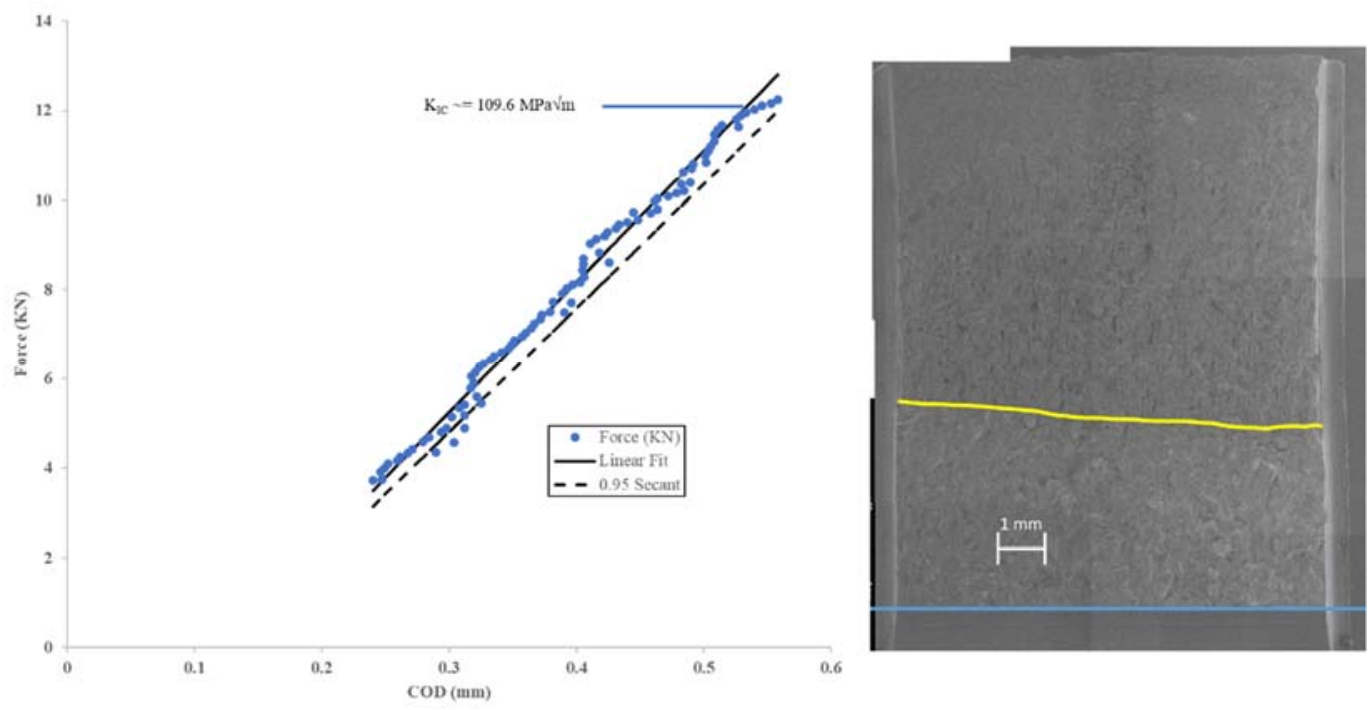

Figure 4-17: Force vs. displacement and fracture surface for 10A0002C03 (alloy X-750, 1.49 dpa) specimen. 


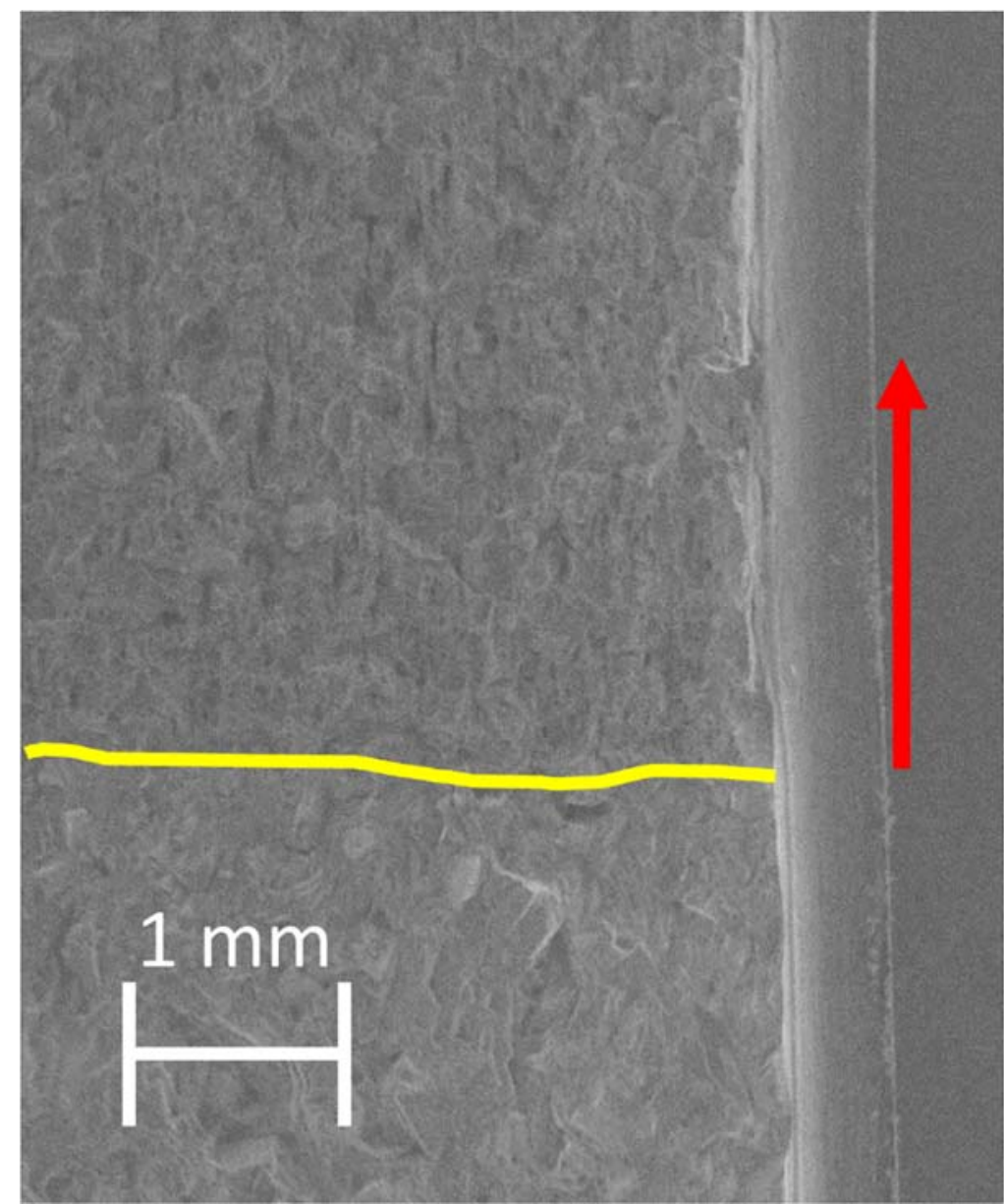

Figure 4-18: Closeup of fracture surface for 10A0002C03 (alloy X-750, $1.49 \mathrm{dpa}$ ).

\subsubsection{Summary of alloy $X-750$ fracture toughness testing}

An overlay plot of the J-R curves for the four lower fluence alloy X-750 fracture toughness tests with a baseline J-R curve from [1] shows an expected reduction in fracture toughness, at least for the medium fluence specimens (Figure 4-19). As suggested in Section 4.2.2.1, the apparent increase in fracture toughness for the lowest fluence specimens is likely an artifact of the difference in constraint between the 0.4T-CT specimen used for the irradiated material fracture toughness tests and the 0.5T-CT specimen employed for baseline testing. J-R curves for the highest fluence specimens are irrelevant, since there was no stable tearing exhibited, and are not included in the overlay plot. Table 4-5 contains all of the fracture toughness data obtained for alloy X-750 during this testing, as well as some of the baseline results from [1]. The most significant reduction in fracture toughness is observed between the medium fluence $(\sim 0.3$ dpa) and high fluence ( $\sim 1.5 \mathrm{dpa})$ tests, as the fracture process transitioned from one of ductile tearing to nearly linear elastic fracture. Except in the case of the highest fluence specimens, caution is advised in interpreting results from single point $\left(\mathrm{J}_{\mathrm{Q}}\right.$ or $\left.\mathrm{K}_{\mathrm{Q}}\right)$ values of fracture toughness as they are estimated as the intersection of the $0.2-\mathrm{mm}$ blunting line and the log fit of J-R data, and are thus subject to testing perturbations. It is advisable to consider the general area under the J-R curve as a more consistent measure of differences in fracture toughness capacity. However, roughly speaking, if average $\mathrm{K}_{\mathrm{IQ}}$ and $\mathrm{K}_{\mathrm{IC}}$ values are used, reductions in fracture toughness compared to the baseline for alloy X-750 exposed to neutron irradiation are 4,18 , and $41 \%$ for the $0.086,0.3$, and 1.5 dpa damage levels, respectively. 


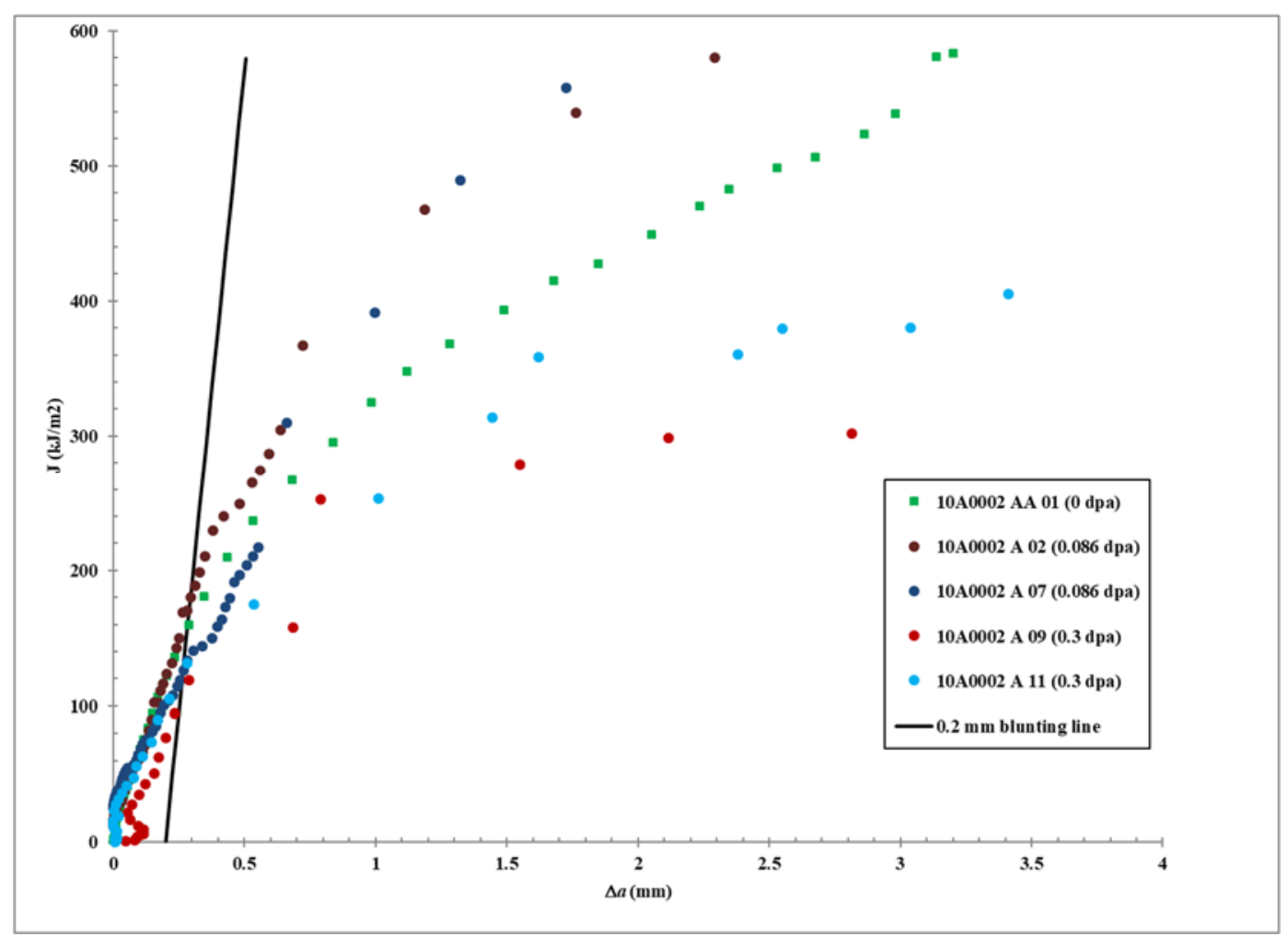

Figure 4-19: Overlay plot of alloy $\mathrm{X}-750 \mathrm{~J}-\mathrm{R}$ curves for baseline, low, and medium fluence tests at $288^{\circ} \mathrm{C}$.

Table 4-5: Fracture toughness estimates for Alloy X-750.

\begin{tabular}{|c|c|c|c|c|}
\hline Alloy X-750 & & & $J_{Q}$ & $\mathrm{~K}_{\mathrm{JO}} / \mathrm{K}_{\mathrm{IC}}$ \\
\hline & Size & Dose $(\mathrm{dpa})$ & $\left(\mathrm{kJ} / \mathrm{m}^{2}\right)$ & $(\mathrm{MPa}-\mathrm{Vm})$ \\
\hline 10A0002AA01 & $0.5 \mathrm{~T}$ & 0 & 163.5 & 192.8 \\
\hline 10A0002AA02 & $0.5 \mathrm{~T}$ & 0 & 160.8 & 190 \\
\hline 10A0002A02 & $0.4 \mathrm{~T}$ & 0.0858 & 181.5 & 203 \\
\hline 10A0002A07 & $0.4 \mathrm{~T}$ & 0.0864 & 121 & 165 \\
\hline 10A0002A09 & $0.4 \mathrm{~T}$ & 0.306 & 99 & 150 \\
\hline 10A0002A11 & $0.4 \mathrm{~T}$ & 0.307 & 121 & 165 \\
\hline 10A0002B08 & $0.4 \mathrm{~T}$ & 1.538 & $\mathrm{~N} / \mathrm{A}$ & 112 \\
\hline 10A0002B10 & $0.4 \mathrm{~T}$ & 1.538 & $\mathrm{~N} / \mathrm{A}$ & 115 \\
\hline 10A0002C03 & $0.4 \mathrm{~T}$ & 1.49 & $\mathrm{~N} / \mathrm{A}$ & 110 \\
\hline
\end{tabular}

\subsubsection{XM-19 Fracture Toughness Tests}




\subsubsection{Lowest fluence $X M-19$ fracture toughness}

Two XM-19 specimens irradiated to the lowest fluence were tested for fracture toughness. Specimens 10A0001A02 and 10A0001A07 both accumulated approximately 0.08 dpa of irradiation damage. From Table 3-3, the estimated average irradiation temperatures for these specimens were $327^{\circ} \mathrm{C}$ and $339{ }^{\circ} \mathrm{C}$, respectively. Following fatigue pre-cracking, both specimens were loaded monotonically at an initial rate of $1.0 \times 10^{-4} \mathrm{~mm} / \mathrm{s}$, then increased to a rate of $1.0 \times 10^{-3} \mathrm{~mm} / \mathrm{s}$ for the remainder of the tests once the approximate peak force was reached. Specimens were post-test fatigued with an applied $\mathrm{K}_{\max }<$ $50 \%$ of the final measured load to mark the final crack front.

As seen in Figure 4-20 and Figure 4-21, crack growth was primarily ductile in nature, with a combination of dimple rupture networks and micro-void coalescence. Although crack tunneling was not excessive, it was extensive enough in both cases to invalidate the tests according to ASTM Standard E 1820-09 [4]. Initial waviness in the test record for specimen 10A0001A02 is due to an oscillating temperature controller. Both specimens exceeded the maximum allowable $\mathrm{J}\left(\mathrm{J}_{\text {limit }}\right)$, which is a function of flow stress and net thickness, so only a portion of the data in the region between the $0.15-\mathrm{mm}$ and $1.5-\mathrm{mm}$ offset lines was used to fit a power law for $\mathrm{J}_{\mathrm{Q}}$ estimation. As baseline testing for this project was conducted with $19.3 \%$ CW XM-19, a comparison cannot be drawn between these results and the baseline results; however, fracture toughness tests on unirradiated material of the same heat were conducted by Andresen and Mora in [13]. Compared to the $\mathrm{K}_{\mathrm{JIC}}$ value of $296 \mathrm{MPaVm}$ cited in this reference, there is an approximate $6 \%$ reduction in fracture toughness if the average $\mathrm{K}_{\mathrm{JQ}}$ from the two tests conducted is used. This is consistent with the increase in tensile strength seen in the tensile tests.

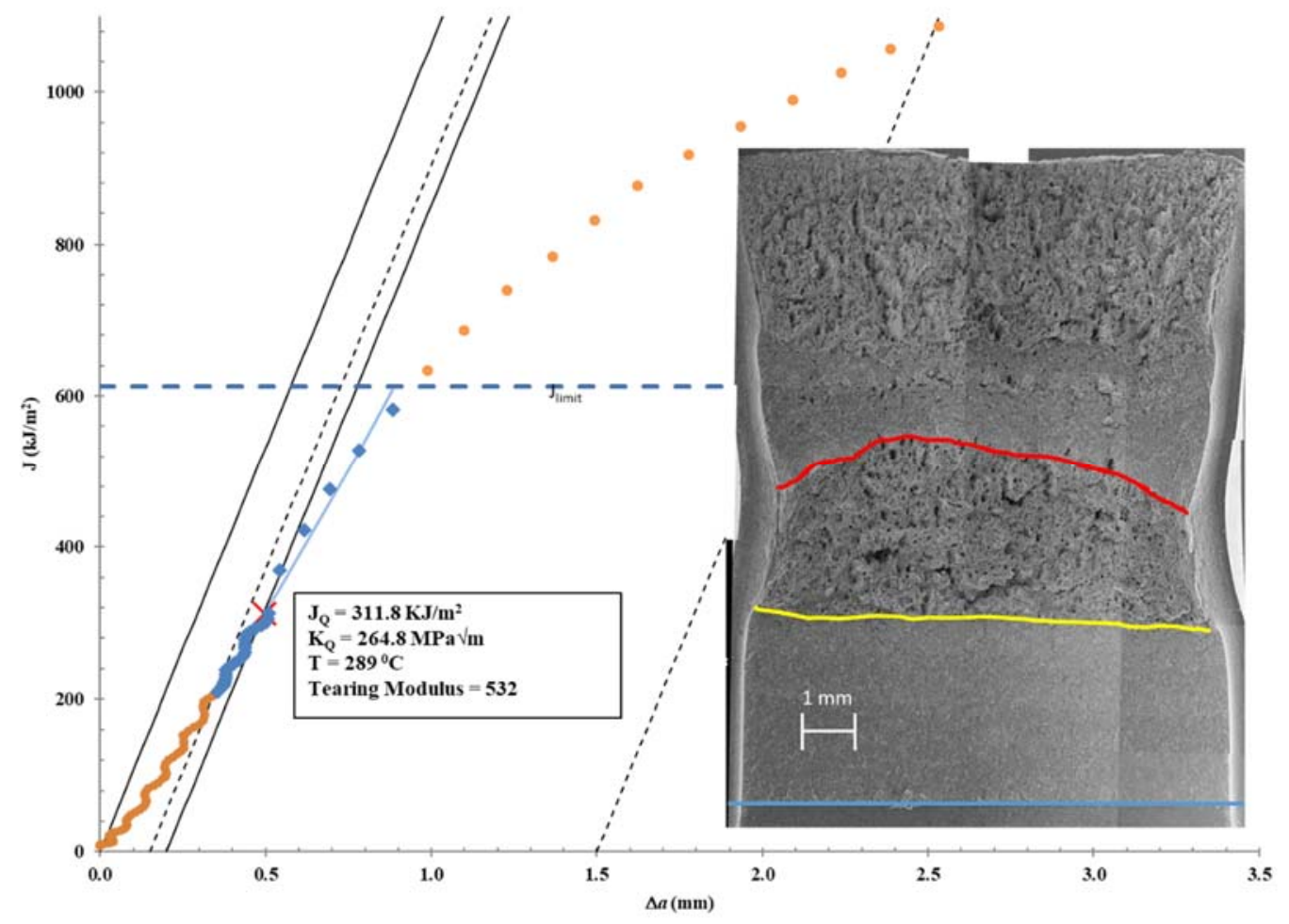

Figure 4-20: J-R curve and fracture surface for 10A0001A02 (XM-19, $0.08 \mathrm{dpa})$ specimen. 


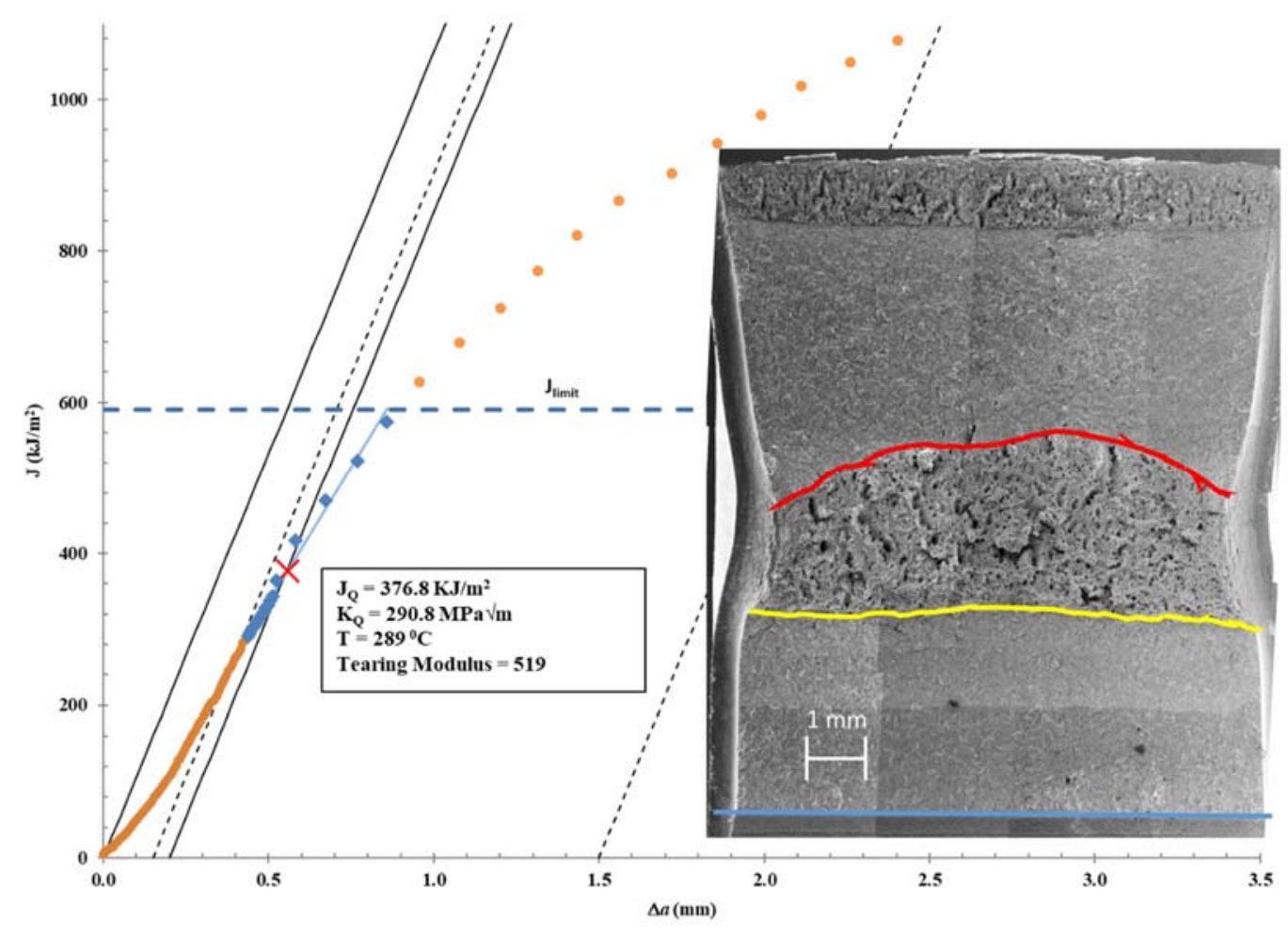

Figure 4-21: J-R curve and fracture surface for 10A0001A07 (XM-19, $0.08 \mathrm{dpa}$ ) specimen.

\subsubsection{Medium fluence XM-19 fracture toughness}

Two XM-19 specimens irradiated to medium fluence were tested in this project. Specimens 10A0001B01 and 10A0001B02 accumulated approximately 0.288 and 0.291 dpa of damage, respectively, with average temperatures of $329^{\circ} \mathrm{C}$ and $350{ }^{\circ} \mathrm{C}$ and maximum short-duration irradiation temperatures of $338^{\circ} \mathrm{C}$ and $360^{\circ} \mathrm{C}$, respectively, as seen in Table 3-5. Specimen 10A0001B02 was utilized for a short IASCC test prior to the fracture toughness test; the end of this test is marked with a green line in Figure 4-23. For this test, the crack tip was re-sharpened by fatigue cracking prior to conducting the fracture toughness test. Both specimens were monotonically loaded at a rate of $1.67 \times 10^{-3} \mathrm{~mm} / \mathrm{s}$ following fatigue pre-cracking until the tests were stopped after sufficient crack growth. Following completion of the tests, the specimens were post-test fatigued to mark the extent of crack growth by fatiguing to final failure with an applied $\mathrm{K}_{\max }<50 \%$ of the final measured testing $\mathrm{K}$.

The two medium fluence XM-19 specimens behaved very similarly to the low fluence specimens with ductile fracture characteristics and stable crack propagation. As with the low fluence specimens, the maximum allowable $\mathrm{J}\left(\mathrm{J}_{\text {limit }}\right)$ was reached, so only a portion of the data between the $0.15-\mathrm{mm}$ and $1.5-\mathrm{mm}$ offset lines is used for the power law fit. Also, as with the low fluence tests, the amount of crack tunneling exceeded that allowed to qualify $\mathrm{J}_{\mathrm{Q}}$ as $\mathrm{J}_{\mathrm{IC}}$, as per [4]. Compared to the reference [13] value of $296 \mathrm{MPaVm}$, the average value of $\mathrm{K}_{\mathrm{JQ}}$ for these two specimens $(\sim 247 \mathrm{MPaVm})$ represents an approximate reduction of $16 \%$ reduction in fracture toughness at this medium fluence level. Figure 4-22 and Figure 4-23 show the J-R curves and fracture surfaces for the two medium fluence tests. 


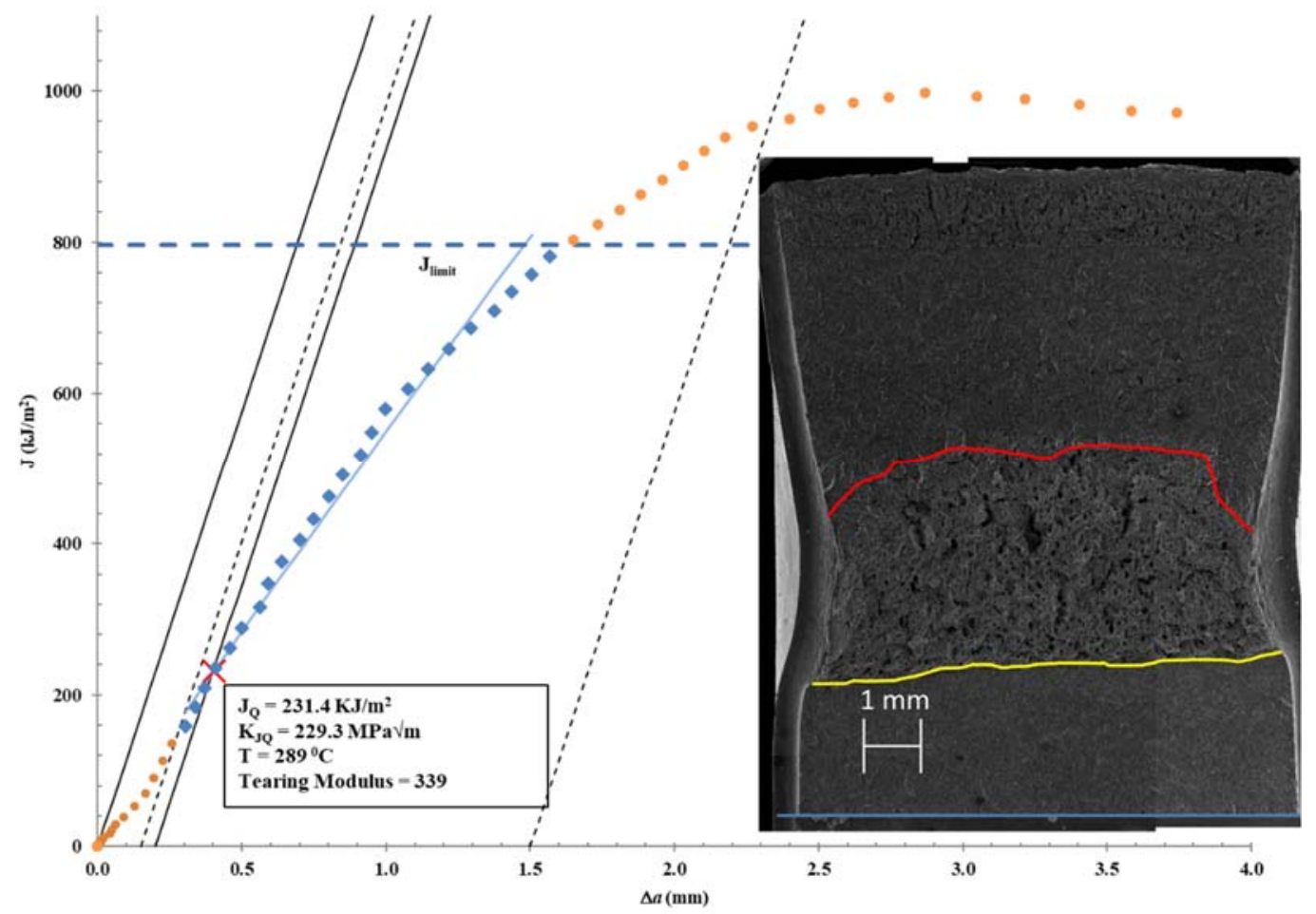

Figure 4-22: J-R curve and fracture surface for 10A0001B01 (XM-19, 0.288 dpa) specimen.

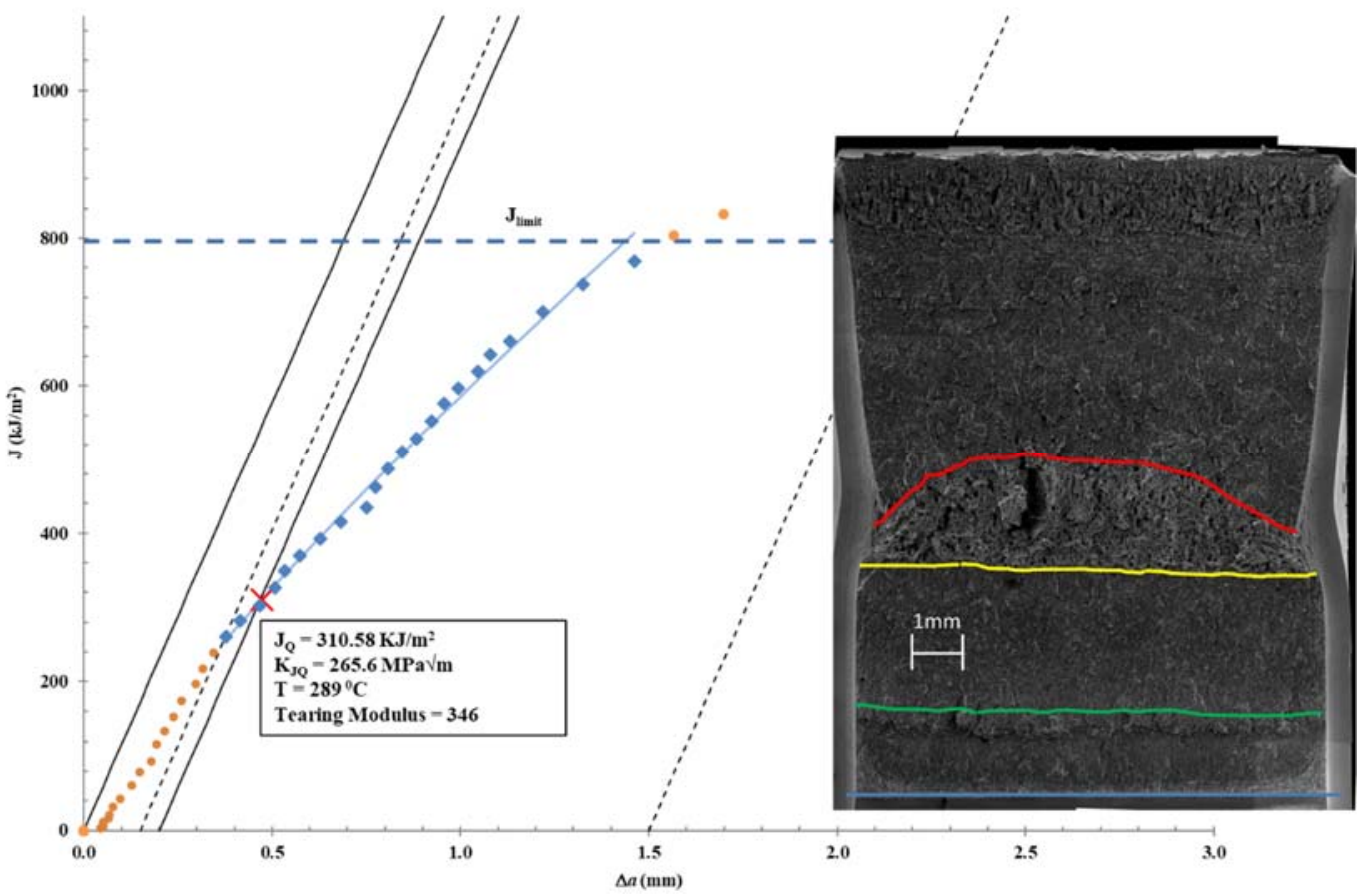

Figure 4-23: J-R curve and fracture surface for 10A0001B02 (XM-19, 0.291 dpa) specimen. 


\subsubsection{Highest fluence XM-19 fracture toughness}

Three fracture toughness tests were conducted on the highest fluence XM-19 material. Specimens 10A001D01 (1.43 dpa), 10A0001D05 (1.47 dpa), and 10A0001E04 (1.41 dpa) were irradiated over the course of two cycles in the EPRI-3 test train. As shown in Table 3-7, the estimated average temperatures for the first and second cycles for specimens 10A0001D01 and 10A0001D05 were $330{ }^{\circ} \mathrm{C}$ and $333{ }^{\circ} \mathrm{C}$ (first cycle) and $261{ }^{\circ} \mathrm{C}$ and $264^{\circ} \mathrm{C}$ (second cycle), respectively, with maximum short-duration temperature excursions of up to $342{ }^{\circ} \mathrm{C}$ and $346^{\circ} \mathrm{C}$ during the first cycle. Specimen 10A0001E04 was irradiated at average temperatures of $301{ }^{\circ} \mathrm{C}$ and $264^{\circ} \mathrm{C}$ during the first and second cycles, with an estimated maximum short-duration temperature of $309^{\circ} \mathrm{C}$ during the first cycle.

Even at this relatively high fluence, ductile fracture dominates, and stable crack propagation is achievable. As with other tests of irradiated XM-19 in the project, the $\mathrm{J}_{\mathrm{Q}}$ values cannot be qualified as $\mathrm{J}_{\mathrm{IC}}$, as per [4], due to excessive crack tunneling and, in the case of 10A0001D01 and 10A0001D05, crooked initial crack fronts. Additionally, the pre-fatigue crack front for specimen 10A0001D05 is highly crooked, and a final J-R test crack front is indiscernible due to a post-fatigue error in which a percentage much higher than the expected $\mathrm{K}_{\max }<50 \%$ of the final measured $\mathrm{K}$ was applied, causing the specimen to rapidly fracture. In comparison to the $296 \mathrm{MPaVm}$ value from reference [13], the average of the $\mathrm{K}_{\mathrm{JQ}}$ values obtained from 10A0001D01 and 10A0001E04 (238 MPaVm) represents an approximate 20\% reduction in fracture toughness. Figure 4-24, Figure 4-25, and Figure 4-26 show the J-R curves and fractographs for the highest fluence XM-19 fracture toughness tests.

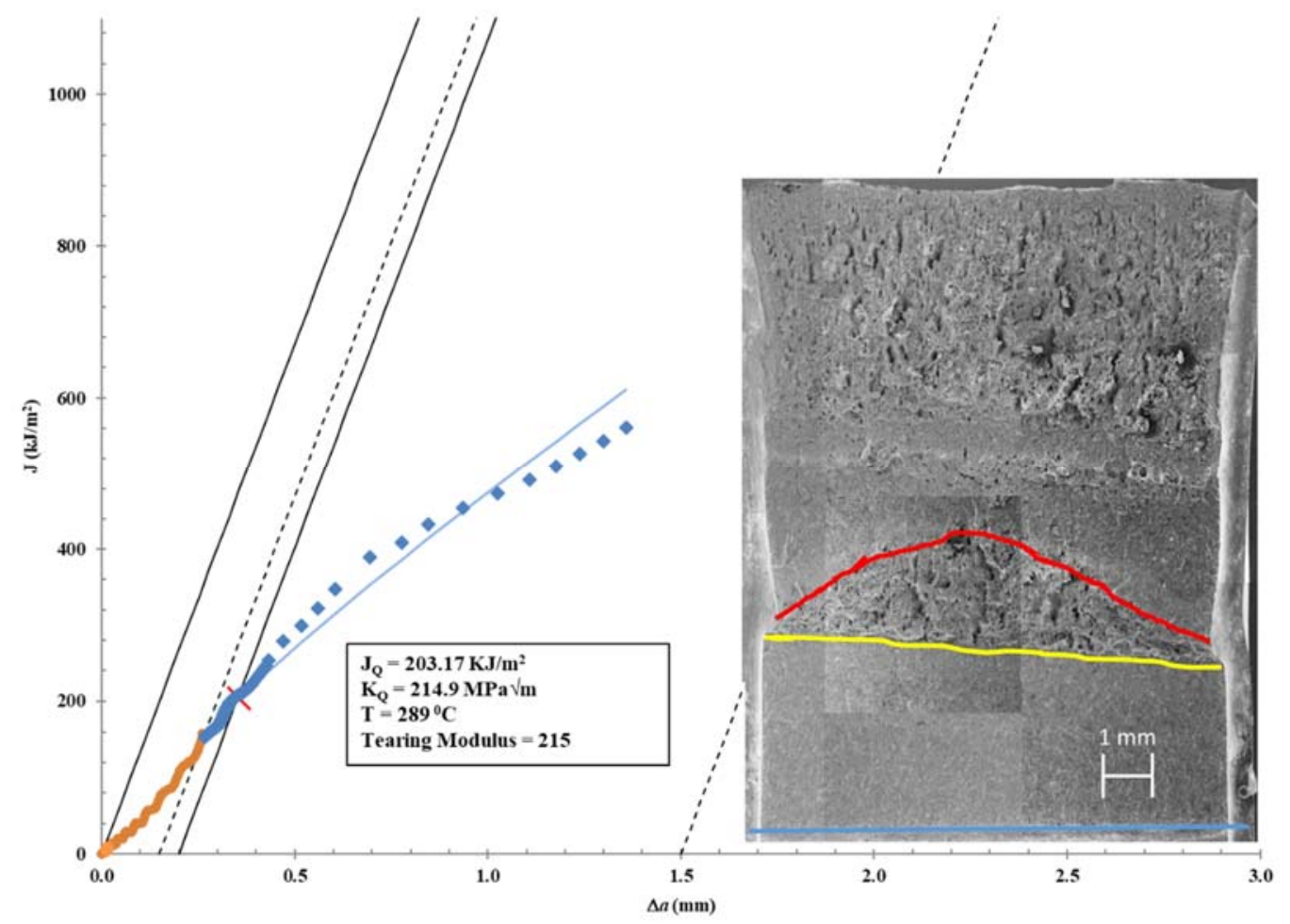

Figure 4-24: J-R curve and fracture surface for 10A0001D01 (XM-19, 1.43 dpa) specimen. 


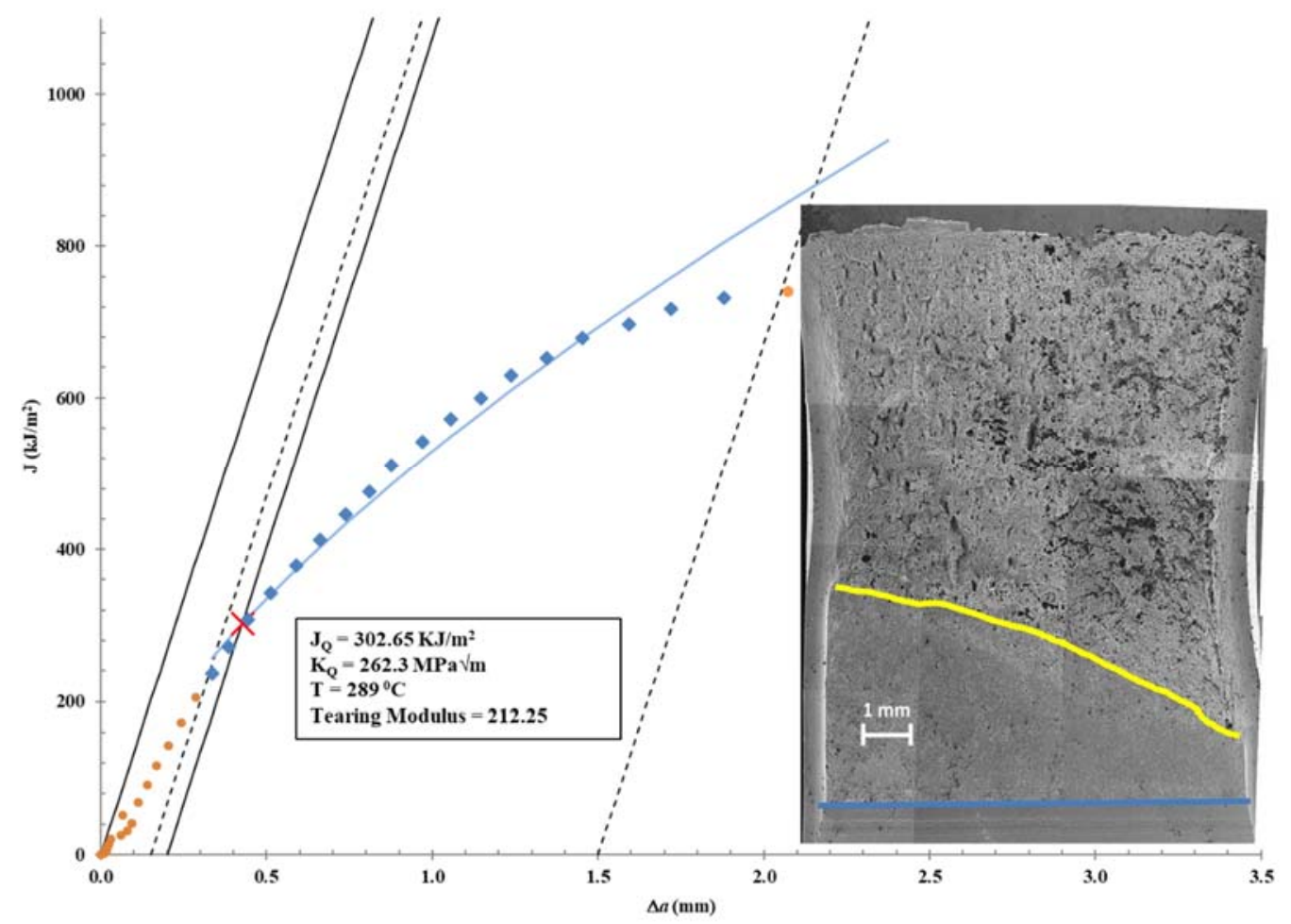

Figure 4-25: J-R curve and fracture surface for 10A0001D05 (XM-19, 1.47 dpa) specimen.

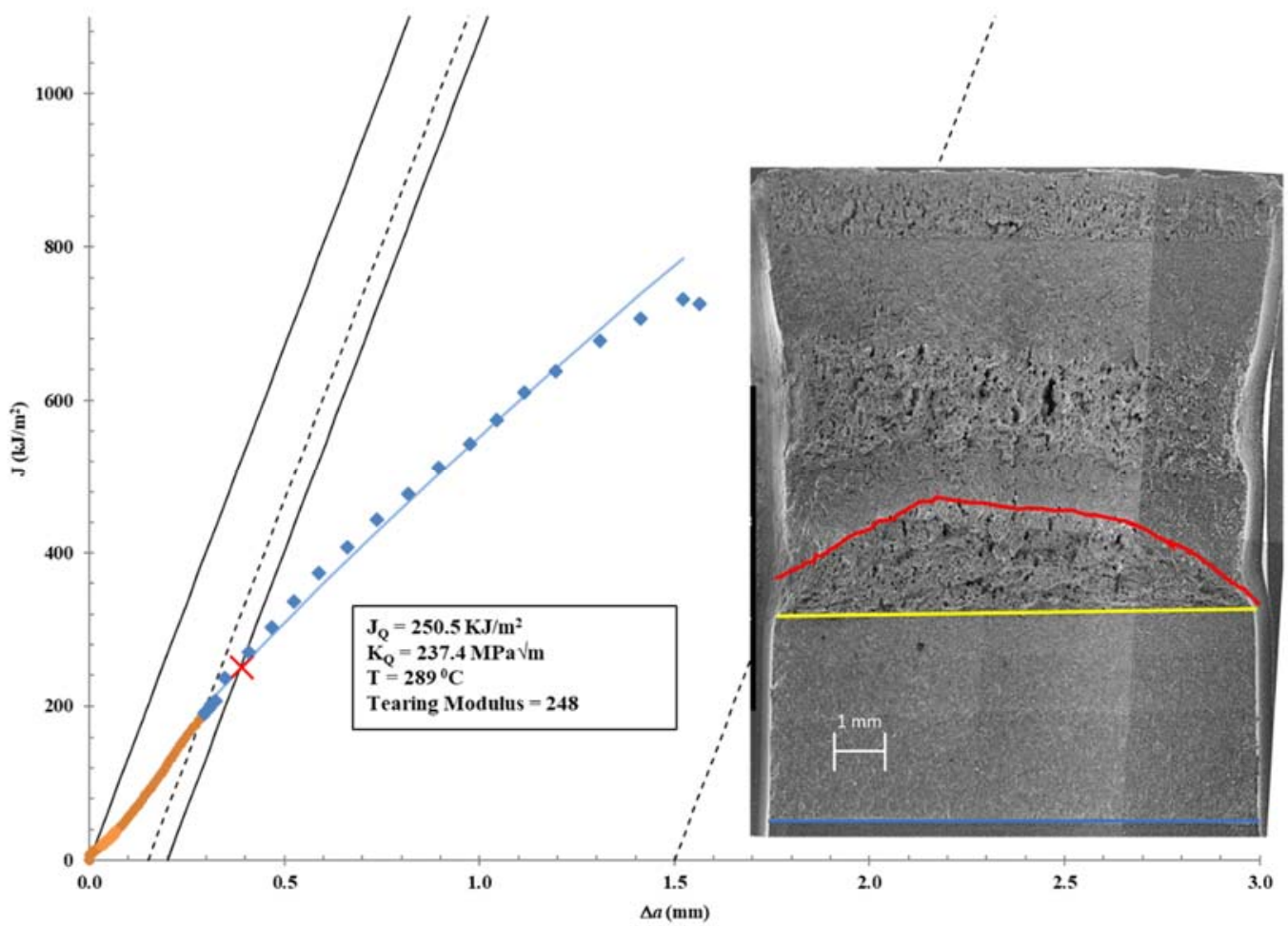

Figure 4-26: J-R curve and fracture surface for 10A0001E04 (XM-19, 1.41 dpa) specimen. 


\subsubsection{Fracture testing summary for XM-19}

Figure 4-27 is an overlay plot of J-R curves for all the neutron-irradiated XM-19 tests conducted for this project, and Table 4-6 is a compilation of $\mathrm{J}_{\mathrm{Q}}$ and $\mathrm{K}_{\mathrm{JQ}}$ results from all tests, along with a value for unirradiated $\mathrm{J}_{\mathrm{IC}} / \mathrm{K}_{\mathrm{IC}}$ in the same material obtained from reference [13]. As expected based on the retention of strain hardening capacity, all three fluence levels - as seen in Section 4.1.3 on tensile testing of XM19 , fracture toughness tests at even the highest fluence exhibited ductile fracture and retained the capacity to support stable crack propagation. A trend in reduction in overall area under the J-R curve, along with reductions in measured $\mathrm{J}_{\mathrm{Q}}$ as a function of irradiation damage, was exhibited. Although compared to the baseline value of $296 \mathrm{MPaVm}$ from [13], the low, medium, and high fluence $\mathrm{K}_{\mathrm{JQ}}$ averages are 6, 16, and $20 \%$ lower, respectively, it should be noted that a high value of fracture toughness remains even at the high damage level of $\sim 1.4 \mathrm{dpa}$, in which the fracture toughness curve still exhibits elastic-plastic behavior. As previously noted in this report, the full $\mathrm{J}-\mathrm{R}$ curve is a better indicator of trends in fracture toughness since determination of $\mathrm{J}_{\mathrm{Q}}$ can be affected by experiment noise.

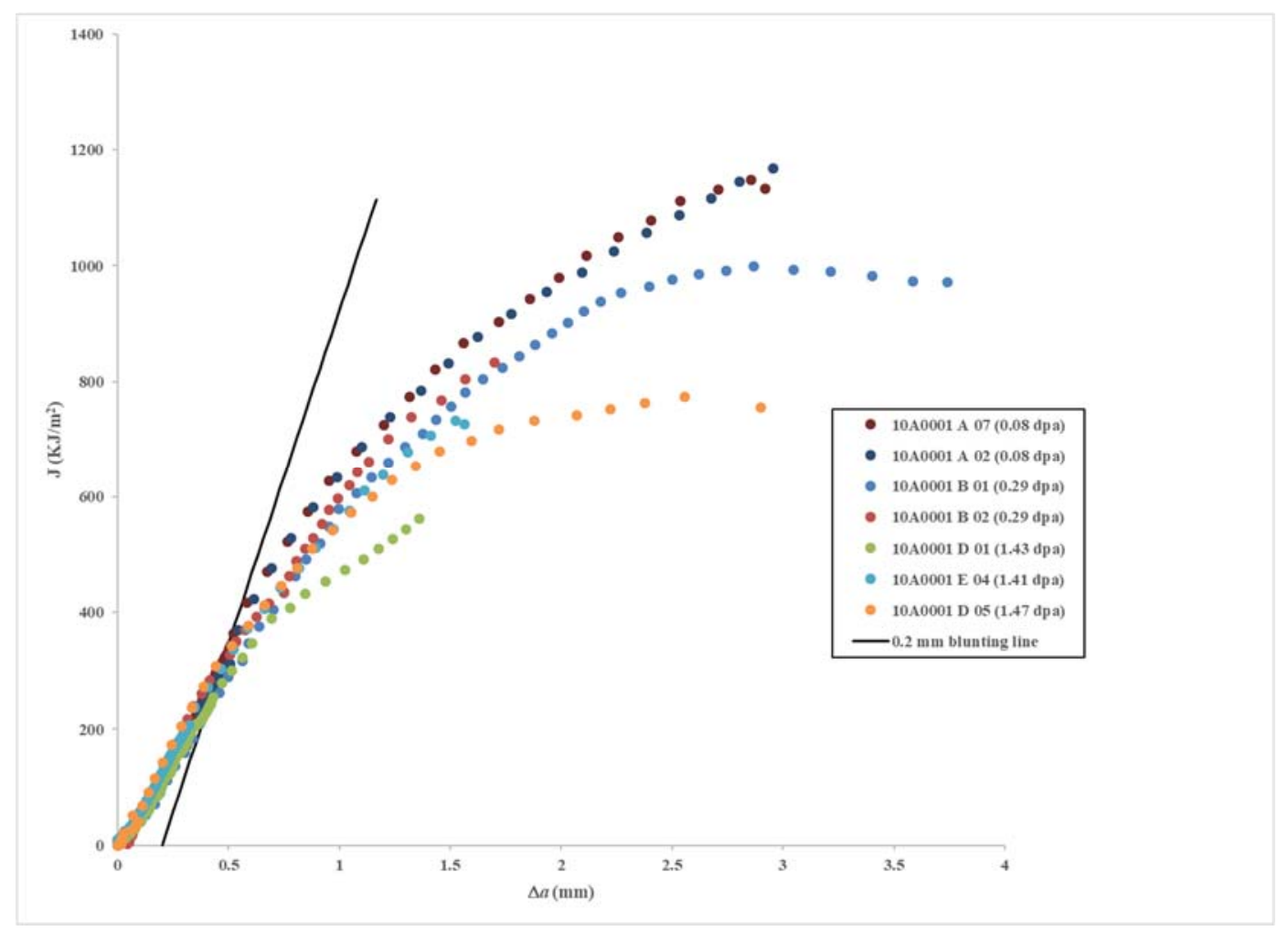

Figure 4-27: Overlay of J-R curves for irradiated XM-19 at $288{ }^{\circ} \mathrm{C}$.

Table 4-6: Fracture toughness estimates for XM-19 (asterisk indicates $\mathbf{J}_{I C} / \mathbf{K}_{I C}$ ).

\begin{tabular}{|c|c|c|c|c|}
\hline $\mathbf{X M - 1 9}$ & & & $\mathrm{J}_{\mathrm{Q}}$ & $\mathrm{K}_{\mathrm{JO}} / \mathrm{K}_{\mathrm{IC}}$ \\
\hline & Size & Dose $(\mathrm{dpa})$ & $\left(\mathrm{kJ} / \mathrm{m}^{2}\right)$ & $(\mathrm{MPa}-\mathrm{Vm})$ \\
\hline C747 [13] & 1.0T & 0 & $388^{*}$ & $296^{*}$ \\
\hline 10A0001A02 & $0.4 \mathrm{~T}$ & 0.079 & 312 & 265 \\
\hline 10A0001A07 & $0.4 \mathrm{~T}$ & 0.08 & 377 & 291 \\
\hline 10A0001B01 & $0.4 \mathrm{~T}$ & 0.29 & 231 & 230 \\
\hline 10A0001B02 & 0.4T & 0.29 & 310 & 266 \\
\hline
\end{tabular}




\begin{tabular}{|c|c|c|c|c|}
\hline 10A0001D05 & $0.4 T$ & 1.47 & 303 & 262 \\
\hline 10A0001D01 & $0.4 T$ & 1.43 & 203 & 215 \\
\hline 10A0001E04 & $0.4 T$ & 1.413 & 251 & 237 \\
\hline
\end{tabular}

\subsubsection{Fracture Toughness Summary}

Although most of the fracture toughness tests violate ASTM E1820-09 [4] requirements, as seen in Table 4-7, engineering values of the fracture toughness still offer at least qualitative comparisons. The exception for this set of tests is the highest fluence alloy X-750 tests, in which neutron damage sufficiently reduced ductility, bringing the specimen size into the valid range. The essence of the validity checks shown in Table 4-7 is that all specimens, except the high fluence alloy X-750 specimens, lacked sufficient constraint to minimize tunneling to within acceptable levels (Requirement 2 in Table 4-7). However, this simply means that the fracture toughness values are not size-independent and are therefore unacceptable for use in engineering calculations. The full J-R curves remain valuable for ascertaining the effect of neutron damage on a component's capacity to function as load bearing, which can be generally surmised as the area under the J-R curve for comparative purposes.

Table 4-7: Table of ASTM validity checks for all fracture toughness tests [4].

\begin{tabular}{|c|c|c|c|c|c|c|c|c|c|c|}
\hline \multirow{2}{*}{ Material } & \multirow{2}{*}{ ID } & \multirow{2}{*}{ Fluence (dpa) } & \multicolumn{2}{|c|}{ Crack size } & \multicolumn{2}{|c|}{ Crack Extension } & \multicolumn{2}{|c|}{ J-R Curve } & \multicolumn{2}{|c|}{ Qualification } \\
\hline & & & 9.1.4.1 & $9.1 .4 .2^{2)}$ & 9.1.5.1 & $9.1 .5 .2^{4)}$ & A9.6.4 & A9.6.6.6 & A.9.9 & A.5.4. $2^{8)}$ \\
\hline$x-750$ & 10A0002A02 & 0.0858 & NO & NO & YES & YES & YES & YES & YES & $\mathrm{N} / \mathrm{A}$ \\
\hline$X-750$ & 10A0002A07 & 0.0864 & NO & NO & NO & YES & YES & YES & YES & $\mathrm{N} / \mathrm{A}$ \\
\hline$x-750$ & 10A0002A09 & 0.306 & YES & NO & NO & YES & YES & YES & YES & $\mathrm{N} / \mathrm{A}$ \\
\hline$X-750$ & 10A0002A11 & 0.307 & YES & NO & NO & YES & YES & YES & YES & $\mathrm{N} / \mathrm{A}$ \\
\hline$x-750$ & 10A0002B08 & 1.538 & YES & $\mathrm{N} / \mathrm{A}$ & $\mathrm{N} / \mathrm{A}$ & $\mathrm{N} / \mathrm{A}$ & $\mathrm{N} / \mathrm{A}$ & $\mathrm{N} / \mathrm{A}$ & YES & YES \\
\hline$X-750$ & 10A0002B10 & 1.538 & YES & $\mathrm{N} / \mathrm{A}$ & N/A & $\mathrm{N} / \mathrm{A}$ & $\mathrm{N} / \mathrm{A}$ & $\mathrm{N} / \mathrm{A}$ & YES & YES \\
\hline$X-750$ & $10 \mathrm{~A} 0002 \mathrm{CO} 3$ & 1.49 & YES & $\mathrm{N} / \mathrm{A}$ & N/A & $\mathrm{N} / \mathrm{A}$ & $\mathrm{N} / \mathrm{A}$ & $\mathrm{N} / \mathrm{A}$ & YES & YES \\
\hline XM-19 & 10A0001A02 & 0.0789 & YES & NO & YES & YES & YES & YES & NO & $\mathrm{N} / \mathrm{A}$ \\
\hline XM-19 & 10A0001A07 & 0.0795 & YES & NO & YES & YES & YES & YES & NO & $\mathrm{N} / \mathrm{A}$ \\
\hline XM-19 & 10A0001B01 & 0.288 & YES & NO & YES & YES & YES & YES & NO & $\mathrm{N} / \mathrm{A}$ \\
\hline XM-19 & 10A0001B02 & 0.291 & YES & NO & NO & NO & YES & YES & NO & $\mathrm{N} / \mathrm{A}$ \\
\hline XM-19 & 10A0001D01 & 1.43 & YES & NO & NO & YES & YES & YES & YES & $\mathrm{N} / \mathrm{A}$ \\
\hline XM-19 & 10A0001D05 & 1.47 & NO & NO* & YES* & YES* & YES & YES & NO & $\mathrm{N} / \mathrm{A}$ \\
\hline XM-19 & 10A0001E04 & 1.41 & YES & NO & NO & NO & YES & YES & NO & N/A \\
\hline
\end{tabular}

1. 1. None of the nine physical measurements of initial physical crack size shall differ by more than $0.05 \mathrm{~B}$ from the average $\mathrm{a}_{0}$.

2. None of the nine physical measurements of final physical crack size $a_{p}$ shall differ by more than $0.05 B$ from the average $a_{p}$.

3. None of the nine physical measurements of crack extension shall be less than $50 \%$ of the average crack extension.

4. The difference between predicted crack extension, $\Delta \mathrm{a}_{\text {predicted }}$ and measured physical crack extension $\Delta \mathrm{a}_{\mathrm{p}}$ shall not exceed $0.15 \Delta \mathrm{a}_{\mathrm{p}}$ for crack extensions of less than $0.2 \mathrm{~b}_{0}$ (uncracked ligament, $\mathrm{W}-\mathrm{a}_{0}$ ), and the difference shall not exceed $0.03 b_{0}$ thereafter.

5. At least one $\mathrm{J}-\Delta \mathrm{a}$ point shall lie between the $0.15-\mathrm{mm}$ exclusion line and a parallel line with an offset of $0.5 \mathrm{~mm}$ from the construction line. At least one $\mathrm{J}-\Delta \mathrm{a}$ point shall lie between the $0.5-\mathrm{mm}$ line and the $1.5-\mathrm{mm}$ exclusion line.

6. At least five data points must remain between $\Delta \mathrm{a}_{\min }, \Delta \mathrm{a}_{\text {limit, }}$, and $\mathrm{J}_{\text {limit. }}$ 
7. $\mathrm{B}$ and $\mathrm{b}_{0}$ must be $>25 \mathrm{~J}_{\mathrm{Q}} / \sigma_{\mathrm{Y}}$ for $\mathrm{J}_{\mathrm{Q}}$ to qualify as $\mathrm{J}_{\mathrm{IC}}$, or $\mathrm{b}_{0}$ must be $>2.5\left(\mathrm{~K}_{\mathrm{Q}} / \sigma_{\mathrm{Y}}\right)^{2}$ for $\mathrm{K}_{\mathrm{Q}}$ to qualify as $\mathrm{K}_{\mathrm{IC}}$.

8. $\mathrm{P}_{\max } / \mathrm{P}_{\mathrm{Q}}$ must be $\leq 1.10$ for $\mathrm{K}_{\mathrm{Q}}$ to equal $\mathrm{K}_{\mathrm{IC}}$.

Compared with baseline values of fracture toughness, all levels of neutron damage resulted in reduction in fracture toughness for both alloy X-750 and XM-19. In both alloys, an ample amount of load-carrying capacity remains in the materials, even at the highest fluence level, which is assumed to be representative of the end of life for typical BWR internal components in which these materials are employed. In the case of XM-19 in particular, capacity to support ductile crack propagation remains, potentially allowing load relaxation as the crack propagates in certain components.

\section{Irradiation Assisted Stress Corrosion Cracking Tests}

A total of eight IASCC tests were completed for this project: two of each alloy (X-750 and XM-19) from the medium fluence test train (EPRI-2), and two of each alloy from the high fluence test train (EPRI-3). All specimens were 0.4T-CT (Figure 2-1) and machined in the L-T orientation. Since the CGR effect was minimal compared to baseline results [1] in the first medium fluence alloy X-750 IASCC tests conducted, it was decided early on in the project that tests on the lowest fluence (EPRI-1) specimens would be of very low value and therefore would not be conducted.

Platinum current and potential probe leads were spot-welded to the specimen for DCPD measurements of crack length (Figure 4-9). In this technique, the 3A direct current flow through the sample is reversed about once per second, primarily to reduce measurement errors associated with thermocouple effects at metal junctions. The computer controlled the current reversal, data acquisition, data averaging, the conversion from measured DCPD to crack length, and crack length to applied stress intensity factor $(\mathrm{K})$ - generally controlling the applied load to maintain a constant maximum applied stress intensity factor $\left(\mathrm{K}_{\max }\right)$. Depending on the test conditions, data were stored in a permanent disk file every 0.1 to $1 \mathrm{~h}$. In addition to the data record number, total elapsed and incremental time, and crack length, the system measured and stored the temperature, current, corrosion potential, effluent dissolved oxygen, effluent conductivity, load, and time/date. Statistical information on temperature and current fluctuations were also recorded. Additionally, messages describing changes in test conditions, etc., are a permanent part of the data record.

A dual clevis-in-clevis design was utilized with zirconia sleeves inserted in the secondary loading pin holes, along with zirconia washers on either side to electrically insulate the primary clevis to which the CT specimens were connected (Figure 4-10). This scheme avoids the complexity associated with inserting pins and zirconia sleeves, and allows easy removal of the specimen-primary-clevis assembly in the event a specimen gets stuck. The lower pull rod was also electrically isolated from the autoclave using a Teflon pressure seal, and from the loading actuator using an insulating washer. Ground isolated instrumentation was used to apply current and read the potentials using the platinum wires spot-welded to the specimen.

Fatigue pre-cracking from the machined notch of 0.5 to $1.0 \mathrm{~mm}$ was performed in the environment, typically at a frequency of $0.5 \mathrm{~Hz}$ at a load ratio $\left(\mathrm{K}_{\min } / \mathrm{K}_{\max }\right) \mathrm{R}$ of $\sim 0.2, \sim 0.4$, and $\sim 0.6$, and a $\mathrm{K}_{\max }$ near the initial stress corrosion cracking (SCC) test value.

Instron servo-hydraulic machines were used to apply loading, and were equipped with a single-stage, slow strain servo valve to ensure optimal (non-noisy) response. All systems are equipped with Model 8800 digital controls to provide improved machine control and full computer interface/control capabilities. The potential drop software interfaced with the Instron 8800 electronics to automate several facets of the load and waveform setup procedure and test control. Automated corrections to load occur only for increases in crack length, thereby avoiding "hunting" problems associated with unnecessary changes in load from noise in the potential drop measurement, and resulting in only very small increases in stress intensity (typically $<0.1 \%$ ) prior to load correction. 
Water chemistry control is vital given the sensitivity of SCC to corrosion potential and anionic activity. De-aerated, demineralized water was introduced to each SCC system through another demineralizer and submicron filter to ensure ultra-high purity $(\approx 0.055 \mu \mathrm{S} / \mathrm{cm})$, then flowed into a glass column (6.4-cm diameter by $183-\mathrm{cm}$ long). A low-pressure pump provided positive pressure to the high pressure pump, drawing water from and recirculating excess water (water that did not go into the high pressure pump) back into the glass column. The autoclave effluent was back-pressure regulated, then measured for conductivity and often for dissolved oxygen. The dissolved gas concentration was controlled by bubbling gas mixtures through the water column. Impurities are added to the glass column using a metering pump controlled by the conductivity meter. Typical impurity additions are, for instance, $0.11 \mu \mathrm{S} / \mathrm{cm} \mathrm{H}_{2} \mathrm{SO}_{4}$, which corresponds to a concentration of $2.08 \times 10^{-7} \mathrm{~N}$, or $10 \mathrm{ppb}$ sulfate. The emphasis was on testing in well-defined conditions of high corrosion potential normal water chemistry $N W C$ (in these tests using $2.5 \mathrm{ppm} \mathrm{O}_{2}$ ) or low corrosion potential hydrogen water chemistry - HWC (in these tests using $60-90 \mathrm{ppb} \mathrm{H}_{2}$ ). NWC and HWC are used synonymously with their more explicit chemistry definitions and with the descriptors "high" and "low" potential throughout the IASCC testing discussion.

All tests were performed in 4-liter stainless steel autoclaves at $288^{\circ} \mathrm{C}$ and about $10.3 \mathrm{MPa}(1,500$ psi). The autoclave effluent water was continuously monitored for solution conductivity using a second conductivity meter and often for dissolved oxygen. A zirconia membrane reference electrode was employed to measure the corrosion potential of the CT specimen. To help ensure the state-of-the-art water chemistry, both influent and effluent water were continuously monitored. Influent water was $\approx 0.055$ $\mu \mathrm{S} / \mathrm{cm}$ unless impurities were intentionally injected. In the absence of impurity additions, the effluent was generally $<0.07 \mu \mathrm{S} / \mathrm{cm}$.

At each of two fluence levels, the SCC growth rate response of replicate specimens was evaluated for alloys X-750 and XM-19, and the replicates are presented in sequence. In all plots, the crack length, CGR, and stress intensity factor $(\mathrm{K})$ were corrected based on the average crack advance using fractography. This correction results in some evolution in K during testing that was not intended but is not surprising.

The highest quality data are obtained by achieving a complete transition from the trans-granular fatigue pre-crack to an intergranular SCC crack, so that the crack behaves as if it had always grown as an SCC crack. This was accomplished in every case.

Additionally, retaining a straight crack front improves the confidence in all of the observed growth rates. DCPD (and most other crack monitoring techniques) are strongly biased by the areas of least crack growth along the crack front, and there is no way to know how unevenness along the crack front evolved during the test. This could result in a somewhat lower indicated growth rate as unevenness developed during part of the test, then an elevated growth rate as the areas that lagged behind in growth began to catch up. There are also ambiguities in the $\mathrm{K}$ value, which is a macroscopic parameter rather than a localized parameter at every point along the crack front. Regions of the crack front that advance deeper do so at a "lower K" because an increasing fraction of the load is carried by the regions that have grown less. For SCC testing, this is always a concern, and not an unusual issue.

The accuracy of DCPD is determined based on incremental growth from the machined notch. Typically, DCPD is quite accurate $(<10 \%$ error) for straight, trans-granular fatigue cracks. Intergranular SCC tends to be more uneven in depth, somewhat or heavily branched, and can involve contact in the wake of the crack - for example, from cracking around enough of the grain perimeter to permit "grain rotation" so contact is retained as the overall crack progresses. Errors of over 1,000\% (i.e., the crack was 10X longer than indicated by DCPD) have been observed by the authors in a few cases for alloy $182 / 82$ weld metals and irradiated stainless steel, and errors of $30-50 \%$ are not uncommon. To help mitigate this issue, the software has "anticipatory correction" to integrate the estimated post-test error into the test control. This rarely eliminates the post-test error but can minimize it so $\mathrm{K}$ does not increase nearly as 
dramatically during the test. The problem is greater when the actual crack is deep, because the change in $\mathrm{K}$ with crack depth is not linear but rises rapidly.

\subsection{Alloy X-750 IASCC Testing Details}

\subsubsection{Medium Fluence Alloy X-750 IASCC CGR Tests}

Specimens 10A0002A08 and 10A0002A10 were the first IASCC tests conducted using the newly installed INL IASCC hot cells. They were irradiated to neutron damage levels of approximately 0.302 and $0.309 \mathrm{dpa}$, respectively and were irradiated at corresponding estimated temperatures of $329-338^{\circ} \mathrm{C}$ and $349-359{ }^{\circ} \mathrm{C}$, respectively. The details of these two IASCC tests follow; in all plots, the blue line tracks crack length vs. time (CGR).

\subsubsection{Specimen 10A0002A08 (0.30 dpa) alloy X-750 IASCC test}

An overview of the response of specimen 10A002A08 is shown in Figure 5-1, and the in-situ fatigue pre-cracking response is shown in Figure 5-2. The IASCC CGR test was begun in $288{ }^{\circ} \mathrm{C}$ water with 2.5 ppm $\mathrm{O}_{2}$ and $10 \mathrm{ppb}$ sulfate $\left(\mathrm{as}_{2} \mathrm{SO}_{4}\right.$ ). In each graph, the label in the outlined box represents the starting condition for that plot. Some graphs show additional $\mathrm{K}$ values, which represent an evolution in $\mathrm{K}$ resulting from the post-test correction.

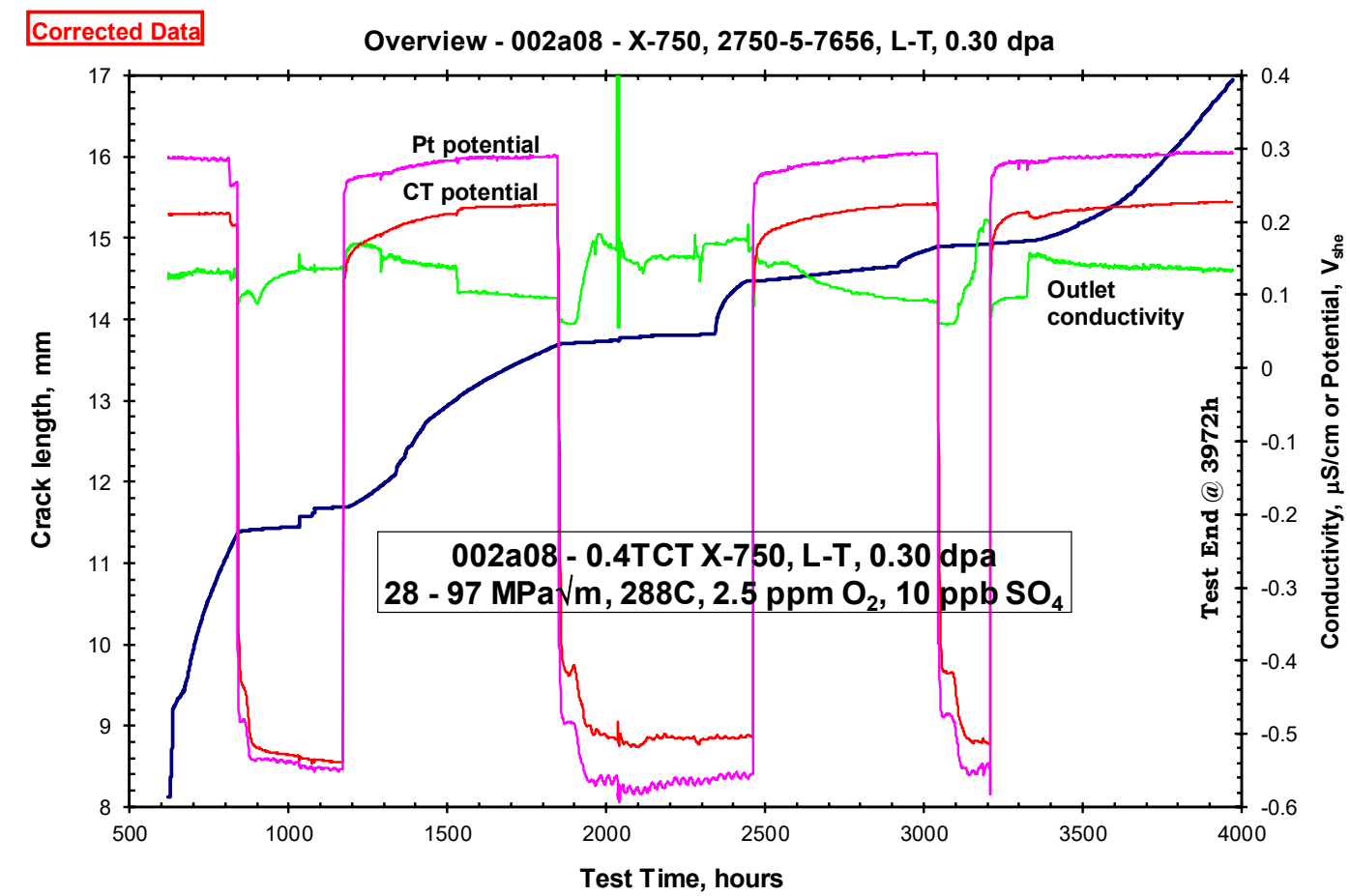

Figure 5-1: IASCC CGR overview of specimen 10A0002A08 of alloy X-750 irradiated to $0.30 \mathrm{dpa}$. 


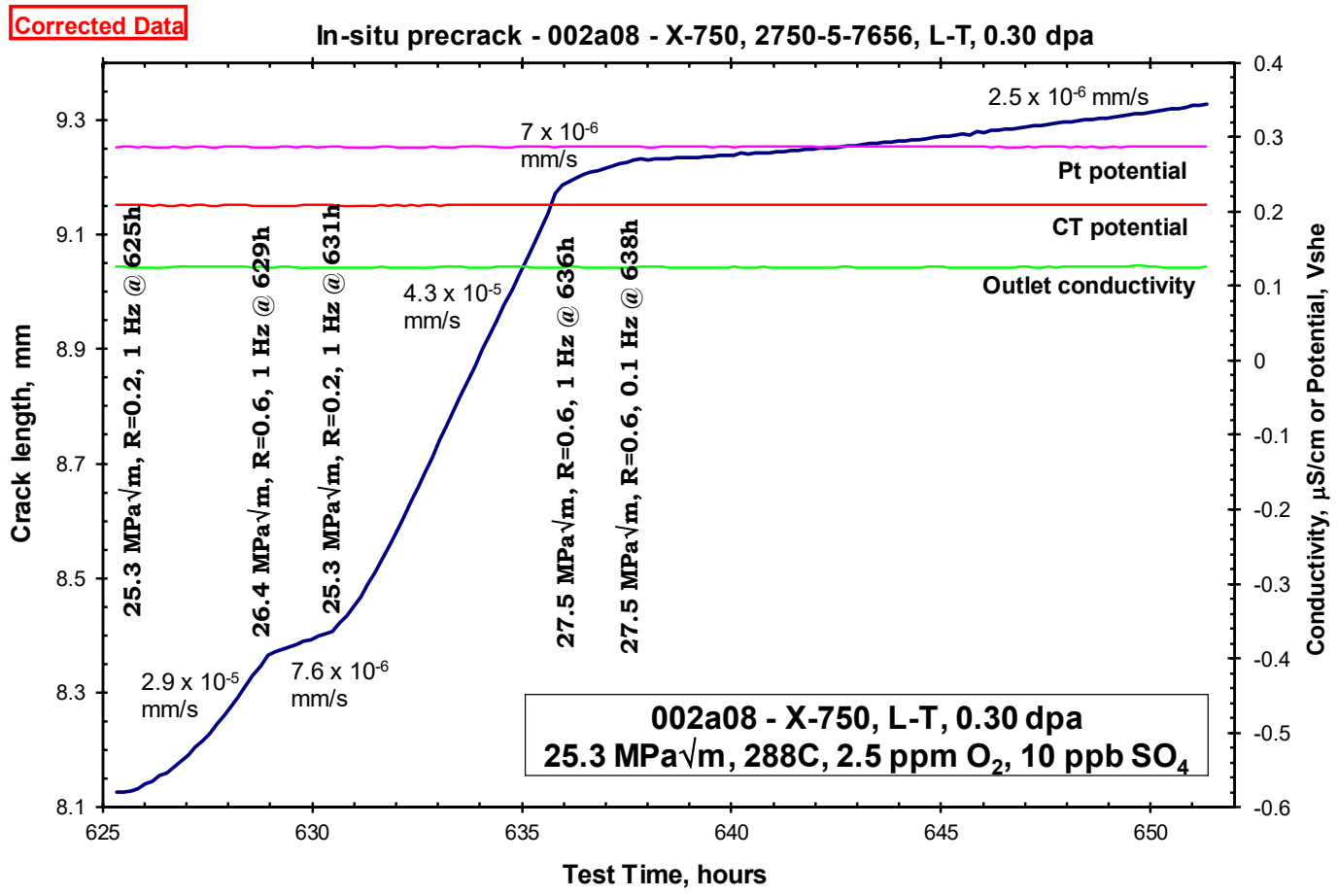

Figure 5-2: In-situ fatigue pre-cracking of specimen $10 \mathrm{A0002} \mathrm{A08}$ of alloy $\mathrm{X}-750$ irradiated to 0.30 dpa.

After fatigue pre-cracking, intergranular transitioning was performed at loading ratio $\mathrm{R}=0.6$ and frequencies 0.01 and $0.001 \mathrm{~Hz}$, then with a 9,000s hold at $\mathrm{K}_{\max }$ (Figure 5-3). The change to constant $\mathrm{K}$ (no cycling) was made at 700 hours, and the subsequent response was well-behaved. The initial growth rate at constant $\mathrm{K}$ decays slightly vs. time, but stabilizes at $2.5 \times 10^{-6} \mathrm{~mm} / \mathrm{s}$.

At 813 hours, a change from $2.5 \mathrm{ppm} \mathrm{O}_{2}$ to $90 \mathrm{ppb} \mathrm{H}_{2}$ was made (Figure 5-4), resulting in a sudden drop in the potential of both the CT specimen and a Pt coupon. The change in growth rate was immediate and dramatic and represents a $\sim 34 \mathrm{X}$ reduction in growth rate. Several loading interruptions occurred in the $\sim 1,000-1,100$ hours timeframe, and subsequently the growth rate further decreased by $\sim 2 \mathrm{X}$. A return to $2.5 \mathrm{ppm} \mathrm{O}_{2}$ at 1,172 hours produced a dramatic increase in growth rate to $8.5 \times 10^{-7} \mathrm{~mm} / \mathrm{s}$, an increase of $23 \mathrm{X}$ over the prior rate measured in $90 \mathrm{ppb} \mathrm{H}_{2}$.

A period of gentle cycling was introduced at 1,338 hours (Figure 5-5), then a return to constant $\mathrm{K}$ (no cycling) was made at 1,436 hours, with the subsequent growth rate almost identical to the prior constant $\mathrm{K}$ growth rate. At 1,530 hours, the injection of $10 \mathrm{ppb}$ sulfate was stopped, and the growth rate decreased somewhat (a reduction of $1.5 \mathrm{X}$ ). This is consistent with a growing body of international data showing that, when well-behaved SCC growth rates are achieved, the effect of such low levels of sulfate or chloride in "corrosion resistant" alloys like stainless steel and nickel alloys is small compared to the effects that change corrosion potential.

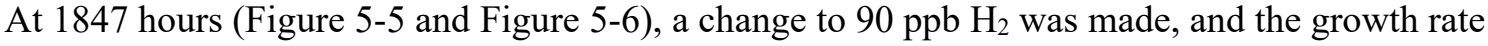
underwent a sudden and significant decrease of $\sim 10 \mathrm{X}$. This reduction is somewhat lower than earlier changes from NWC to HWC, and is partly explained by the fact that $10 \mathrm{ppb}$ sulfate has no effect in HWC but somewhat accelerates the growth rate in NWC. The growth rate eventually slows to $2 \times 10^{-8} \mathrm{~mm} / \mathrm{s}$, which represents a reduction by $\sim 27 \mathrm{X}$.

At 2340 hours, there was a period of cyclic loading at $\mathrm{R}=0.6$, frequency $0.001 \mathrm{~Hz}$, with a 9,000 s hold time at $\mathrm{K}_{\max }$ (Figure 5-7 and Figure 5-8), and the crack grew more rapidly for $\sim 50$ hours, then reached a 
steady state. The step-wise growth of the crack results from each loading cycle, which occurs about every 3 hours $(1,000$ s cycle $+9,000$ s hold $)$. A reasonable interpretation of this response is that the crack front was uneven and, during cycling, became more even. The projection back from the steady-state growth rate (shown by the dashed line in Figure 5-8) indicates a straightening of the crack front by $\sim 0.35 \mathrm{~mm}$. After the loading was returned to constant $\mathrm{K}$ at 2,441 hours, the growth rate returned to the earlier nocycling rate of $2 \times 10^{-8} \mathrm{~mm} / \mathrm{s}$.

At 2,464 hours (Figure 5-7), there was a change from $90 \mathrm{ppb}_{2}$ to $2.5 \mathrm{ppm} \mathrm{O}_{2}$, and the growth rate increased from $2 \times 10^{-8} \mathrm{~mm} / \mathrm{s}$ to $1.2 \times 10^{-7} \mathrm{~mm} / \mathrm{s}$, a factor of only $6 \mathrm{X}$. A period of gentle cycling was introduced, but the growth rate only increased by $\sim 3 X$. At 3,042 hours, a simultaneous change occurred to constant $\mathrm{K}$ and $90 \mathrm{ppb} \mathrm{H}_{2}$, and the growth rate returned to $2 \times 10^{-8} \mathrm{~mm} / \mathrm{s}$.

At 3,209 hours, a change was made from $90 \mathrm{ppb} \mathrm{H}_{2}$ to $2.5 \mathrm{ppm} \mathrm{O}_{2}$, resulting in a rapid increase in the corrosion potential of the CT specimen (Figure 5-9). The CGR response was delayed by about 150 hours, but reached a steady state of about $1.3 \times 10^{-6} \mathrm{~mm} / \mathrm{s}$, which was much higher than at 2,600-2,900 hours, but consistent with data earlier in the test.

It is interesting to note that, at $\mathrm{K}$ values above $70 \mathrm{MPa} \vee \mathrm{m}$, the $\mathrm{SCC}$ growth rate was not $10 \mathrm{X}$ or $100 \mathrm{X}$ higher than earlier in the test at much lower K values-for example, at $31 \mathrm{MPa} \sqrt{\mathrm{m}}$ in Figure 5-4. These 0.4T CT specimens are larger than most irradiated specimens tested internationally (largely stainless steels), but nonetheless exhibit remarkably low sensitivity to K-size validity concerns. Perhaps this is related to the presence of the finely distributed gamma prime precipitation in the microstructure [3].

The test was ended at 3972 hours, and a macro photograph was taken (Figure 5-10). The agreement with DCPD was not unusual for irradiated specimens, with the average intergranular crack depth on the fracture surface being about $56 \%$ higher than was indicated by DCPD. The crack length, CGR, and K data were corrected for all portions of this IASCC test. Table 5-1 is a summary of all the CGRs and related conditions under periods of constant applied $\mathrm{K}$ for this test.

Table 5-1: Constant K IASCC CGRs and conditions for specimen 10A0002A08 of alloy X-750 irradiated to $0.30 \mathrm{dpa}$; blue highlights indicate higher confidence data.

\begin{tabular}{|c|c|c|c|c|c|c|c|}
\hline $\begin{array}{c}\text { Test } \\
\text { Hours }\end{array}$ & $\begin{array}{c}\mathbf{K} \\
(\mathbf{M P a V} \mathbf{m})\end{array}$ & Chemistry & Sulfate & $\begin{array}{c}\text { Outlet } \\
\text { Cond } \\
(\boldsymbol{\mu} \mathbf{S} / \mathbf{c m})\end{array}$ & $\begin{array}{c}\text { Time } \\
\text { Increment } \\
(\mathbf{h r})\end{array}$ & $\begin{array}{c}\text { Growth } \\
\text { Increment } \\
(\mathbf{m m})\end{array}$ & $\begin{array}{c}\text { Average } \\
\text { CGR } \\
(\mathbf{m m} / \mathbf{s})\end{array}$ \\
\hline 700 & 32 & NWC & 10 & 0.13 & 136 & 1.405 & $2.50 \mathrm{E}-06$ \\
\hline 836 & 34 & HWC & 10 & 0.13 & 336 & 0.338 & $5.00 \mathrm{E}-08$ \\
\hline 1172 & 35 & NWC & 10 & 0.165 & 166 & 0.407 & $8.50 \mathrm{E}-07$ \\
\hline 1436 & 40 & NWC & 10 & 0.14 & 94 & 0.278 & $8.10 \mathrm{E}-07$ \\
\hline 1530 & 42 & NWC & 0 & 0.1 & 317 & 0.662 & $5.30 \mathrm{E}-07$ \\
\hline 1847 & 44 & HWC & 0 & 0.1 & 493 & 0.135 & $5.00 \mathrm{E}-08$ \\
\hline 2441 & 54 & HWC & 0 & 0.15 & 23 & 0.001 & $2.00 \mathrm{E}-08$ \\
\hline 2464 & 54 & NWC & 0 & 0.11 & 453 & 0.181 & $1.20 \mathrm{E}-07$ \\
\hline 3042 & 56 & HWC & 0 & 0.08 & 167 & 0.034 & $2.00 \mathrm{E}-08$ \\
\hline 3326 & 68 & NWC & 10 & 0.137 & 646 & 2.018 & $1.30 \mathrm{E}-06$ \\
\hline
\end{tabular}




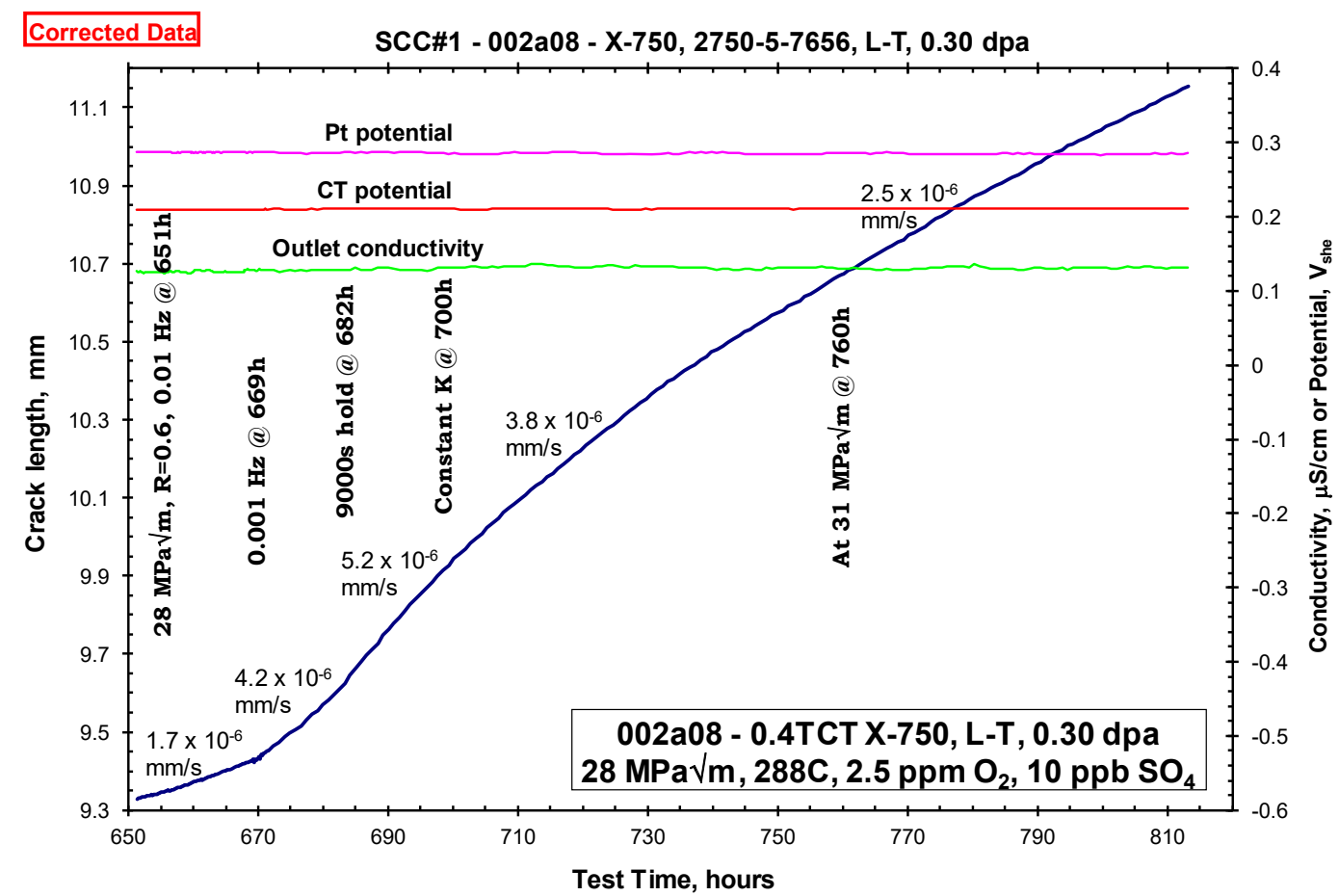

Figure 5-3: Initial IASCC response of specimen $10 \mathrm{~A} 0002 \mathrm{~A} 08$ of alloy X-750 irradiated to $0.30 \mathrm{dpa}$.

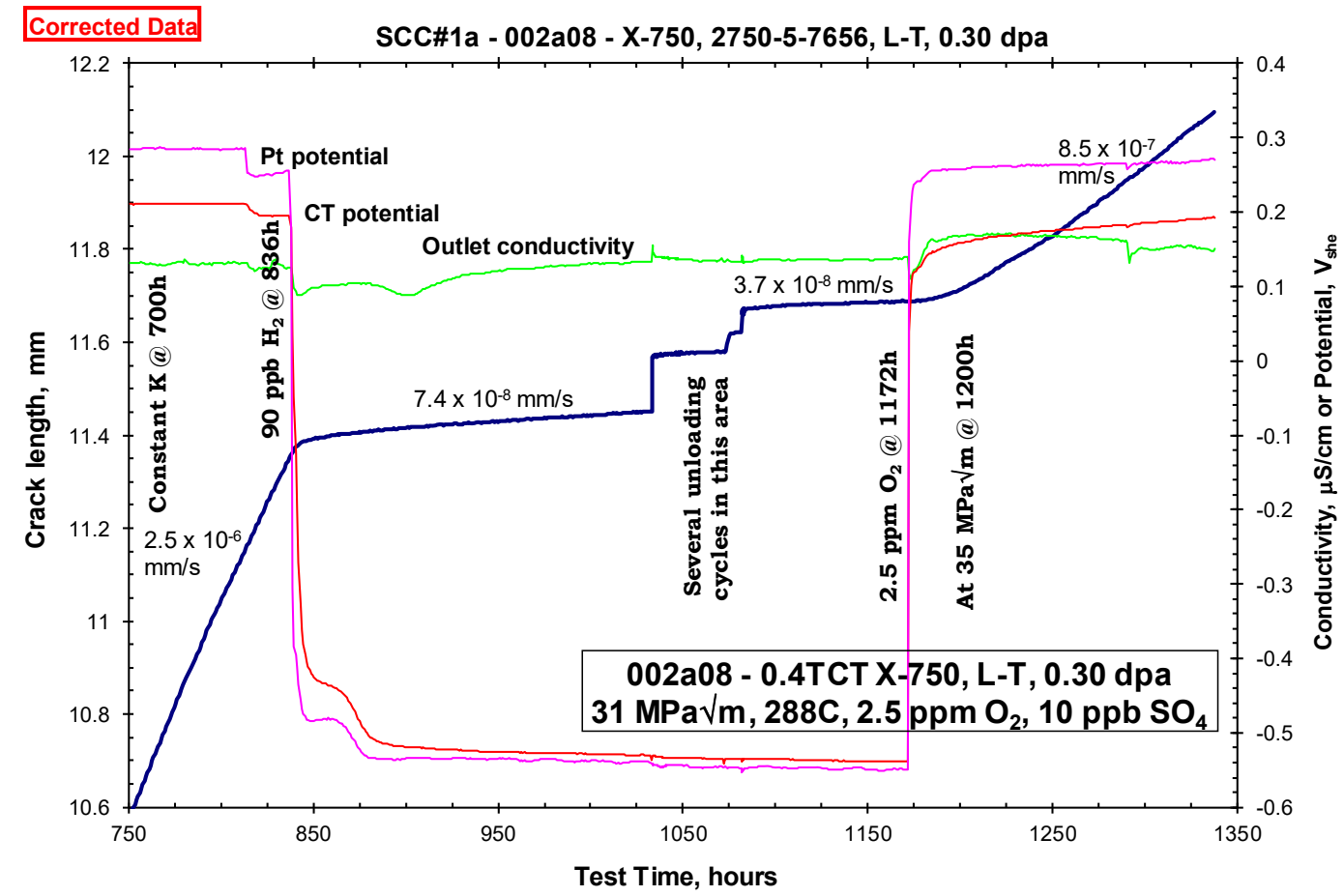

Figure 5-4: IASCC response overview of transition to HWC and return to NWC under constant K for specimen $10 \mathrm{~A} 0002 \mathrm{A08}$ of alloy $\mathrm{X}-750$ irradiated to $0.30 \mathrm{dpa}$. 


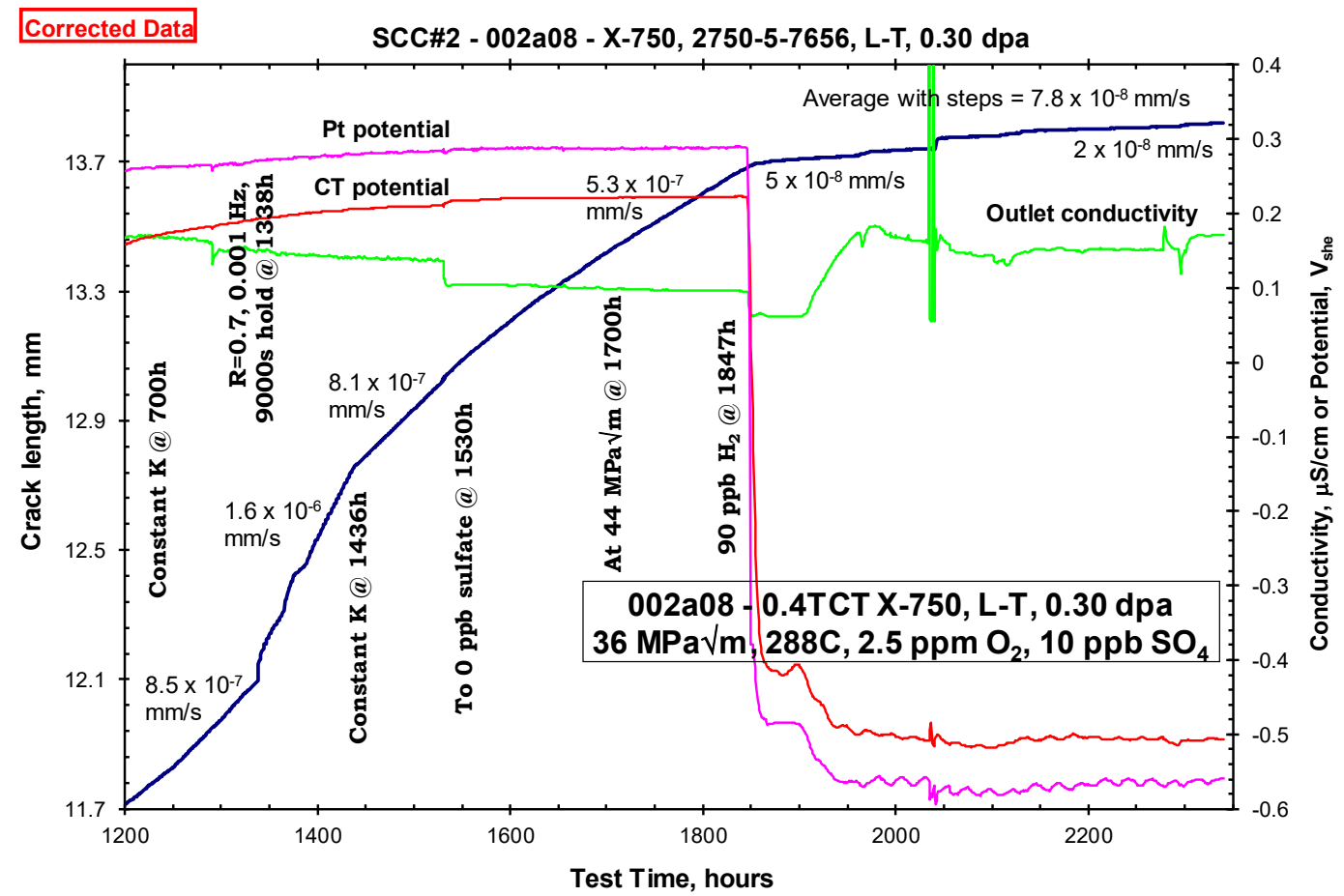

Figure 5-5: IASCC response overview of gentle cycling followed by return to constant $K$ for specimen 10A0002A $\mathrm{A08}$ of alloy X-750 irradiated to 0.30 dpa.

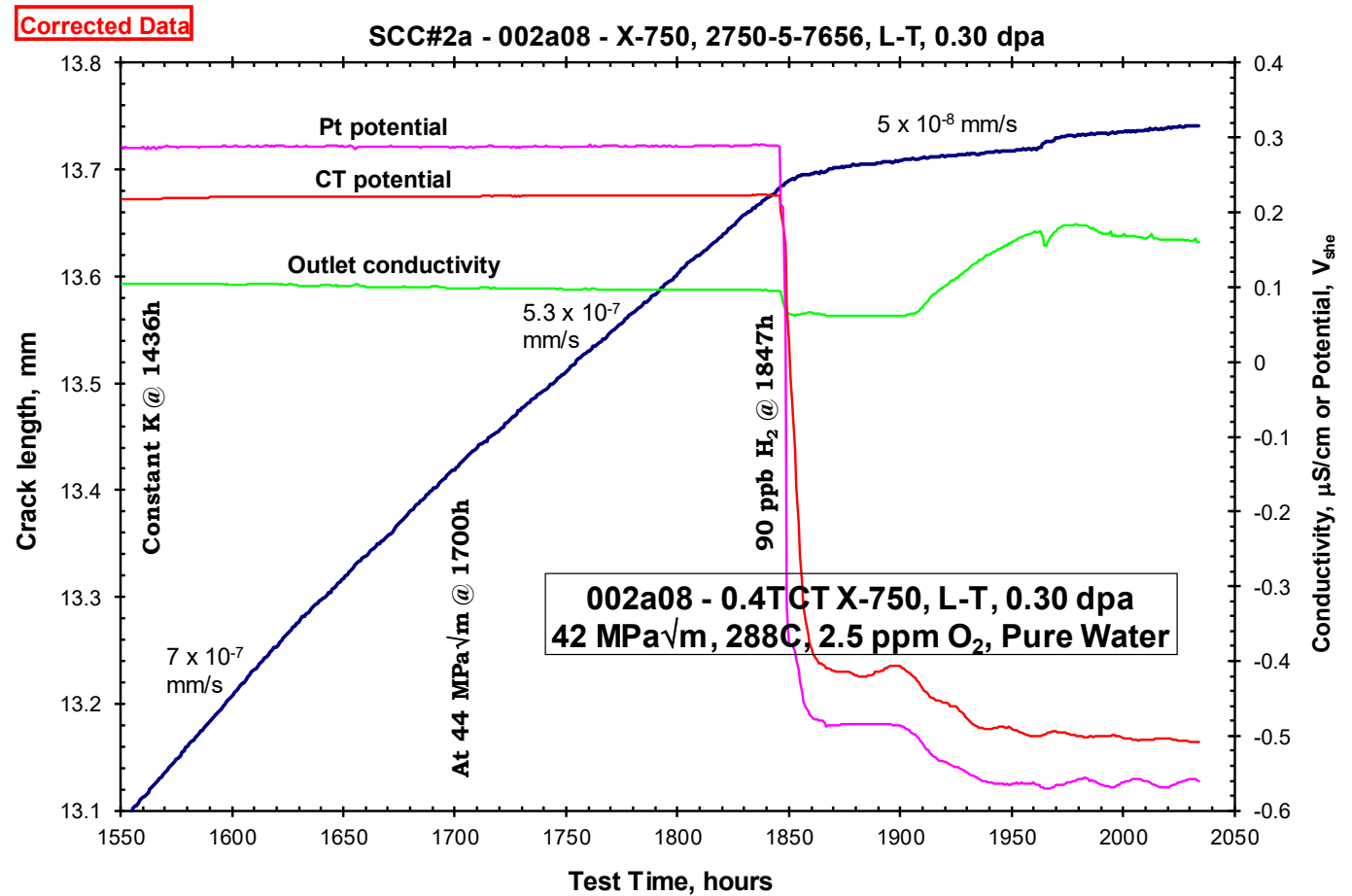

Figure 5-6: Enlarged detail view of Figure 5-5 transition to higher constant $K$ and second transition to HWC for specimen $10 \mathrm{A0002} \mathrm{A08}$ of alloy X-750 irradiated to $0.30 \mathrm{dpa}$. 


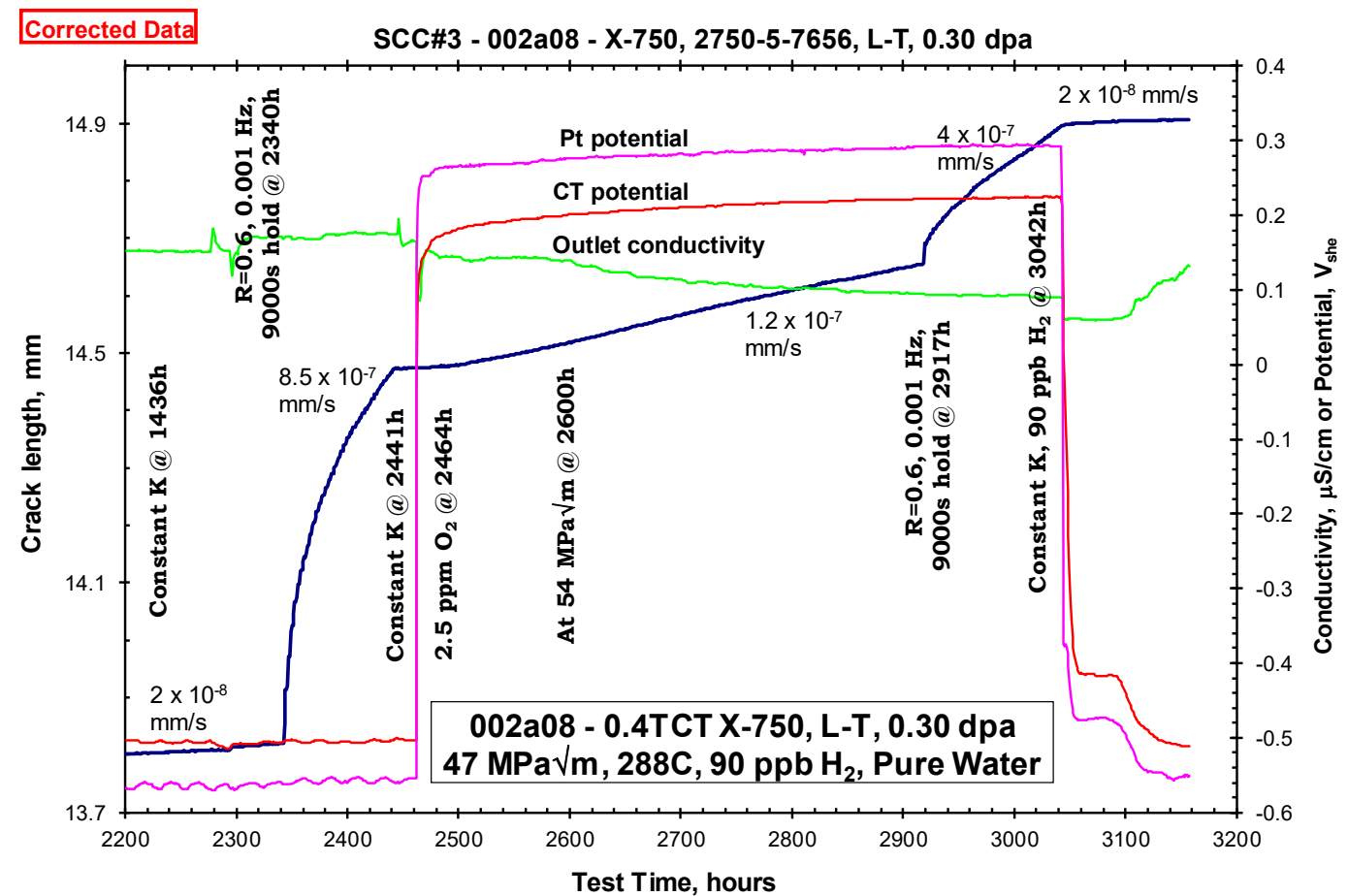

Figure 5-7: IASCC response during and after crack front straightening and at higher $K$ under HWC and NWC for specimen $10 \mathrm{A0002} \mathrm{A08}$ of alloy X-750 irradiated to 0.30 dpa.

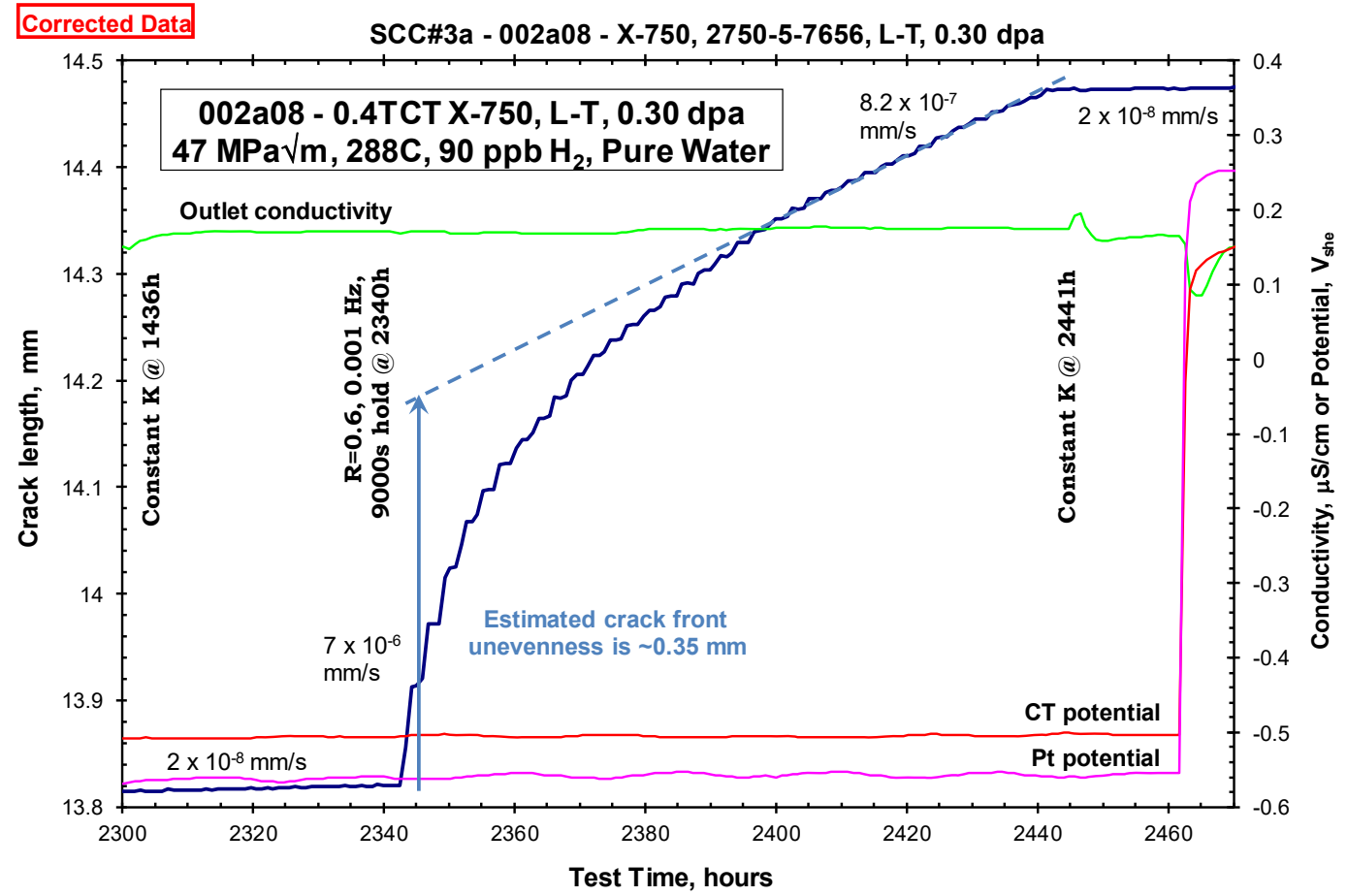

Figure 5-8: Detail view of crack front straightening portion of Figure 5-7 for specimen 10A0002A08 of alloy X-750 irradiated to 0.30 dpa. 


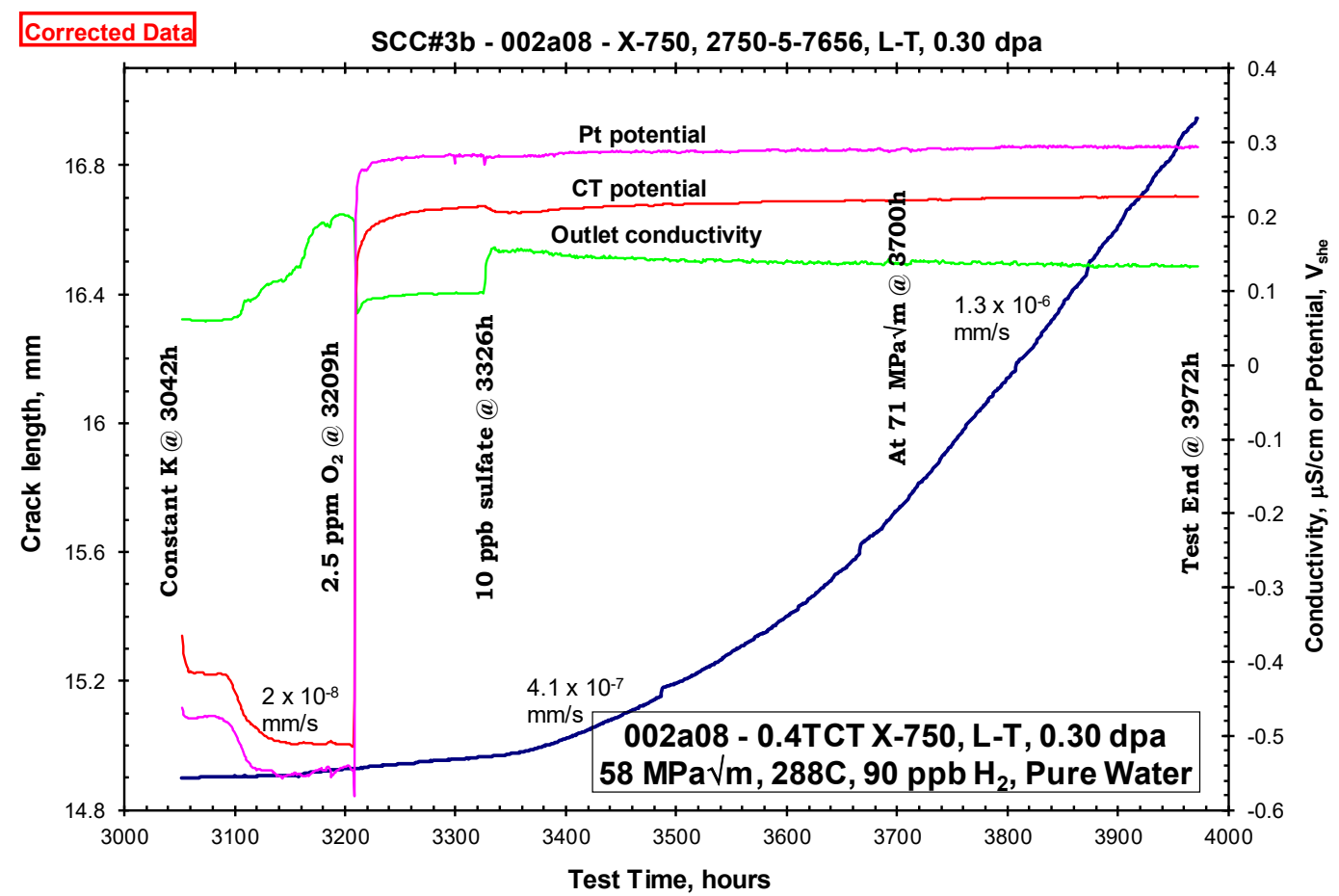

Figure 5-9: Final IASCC test steps for specimen 10A0002A08 of alloy X-750 irradiated to 0.30 dpa.

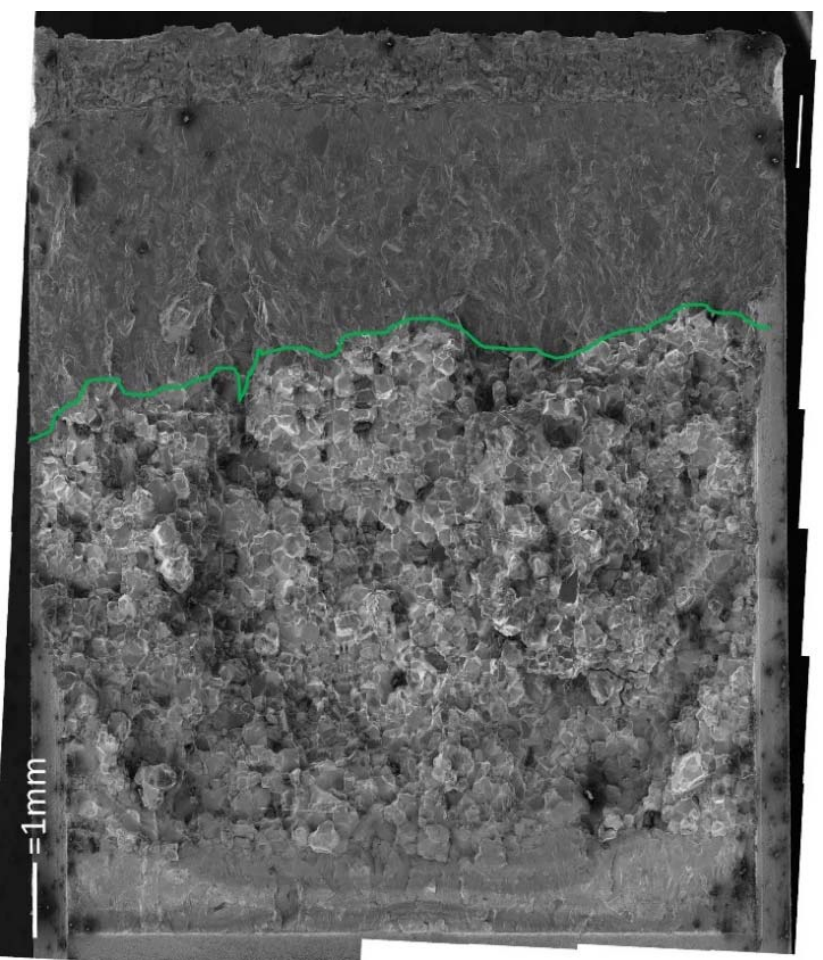

Figure 5-10: SEM macrograph showing fracture surface of specimen 10A0002A08 of alloy X-750 irradiated to $0.30 \mathrm{dpa}$.

\subsubsection{Specimen 10A0002A10 (0.31 dpa) alloy X-750 IASCC test}

This test was begun in $288{ }^{\circ} \mathrm{C}$ water with $2.5 \mathrm{ppm} \mathrm{O}_{2}$ and $10 \mathrm{ppb}$ sulfate $\left(\right.$ as $\left.\mathrm{H}_{2} \mathrm{SO}_{4}\right)$. Figure $5-11$ provides an overview of the response of specimen 10A0002A10 for the entire test, and Figure 5-12 shows 
the in-situ fatigue pre-cracking response. In each graph, the label in the outlined box represents the starting condition for that plot. Some graphs show additional K values, which represent an evolution in $\mathrm{K}$ resulting from the post-test correction, although some intentional changes in $\mathrm{K}$ were also made.

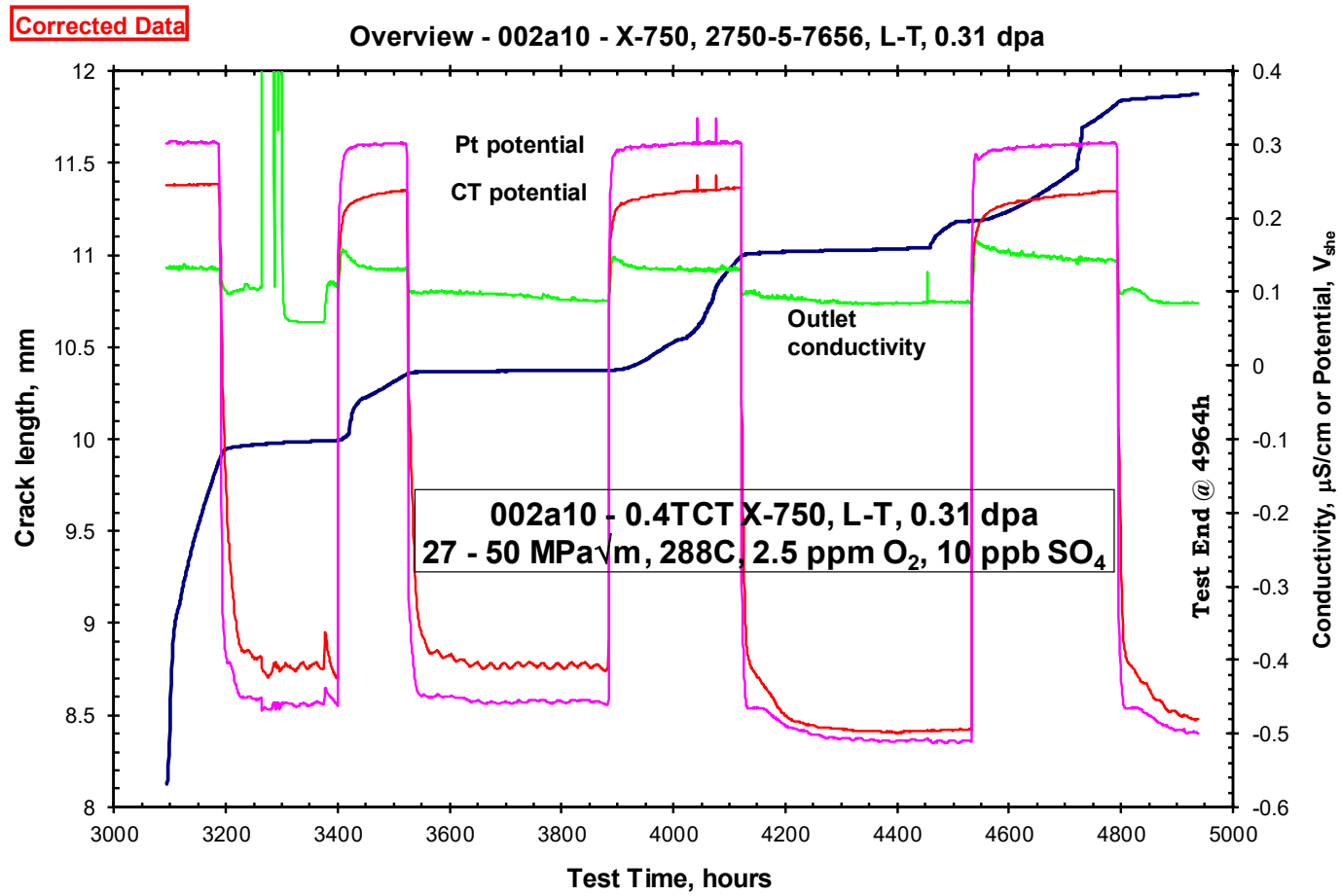

Figure 5-11: IASCC CGR test overview for specimen 10A0002A10 of alloy X-750 irradiated to 0.31 dpa. 


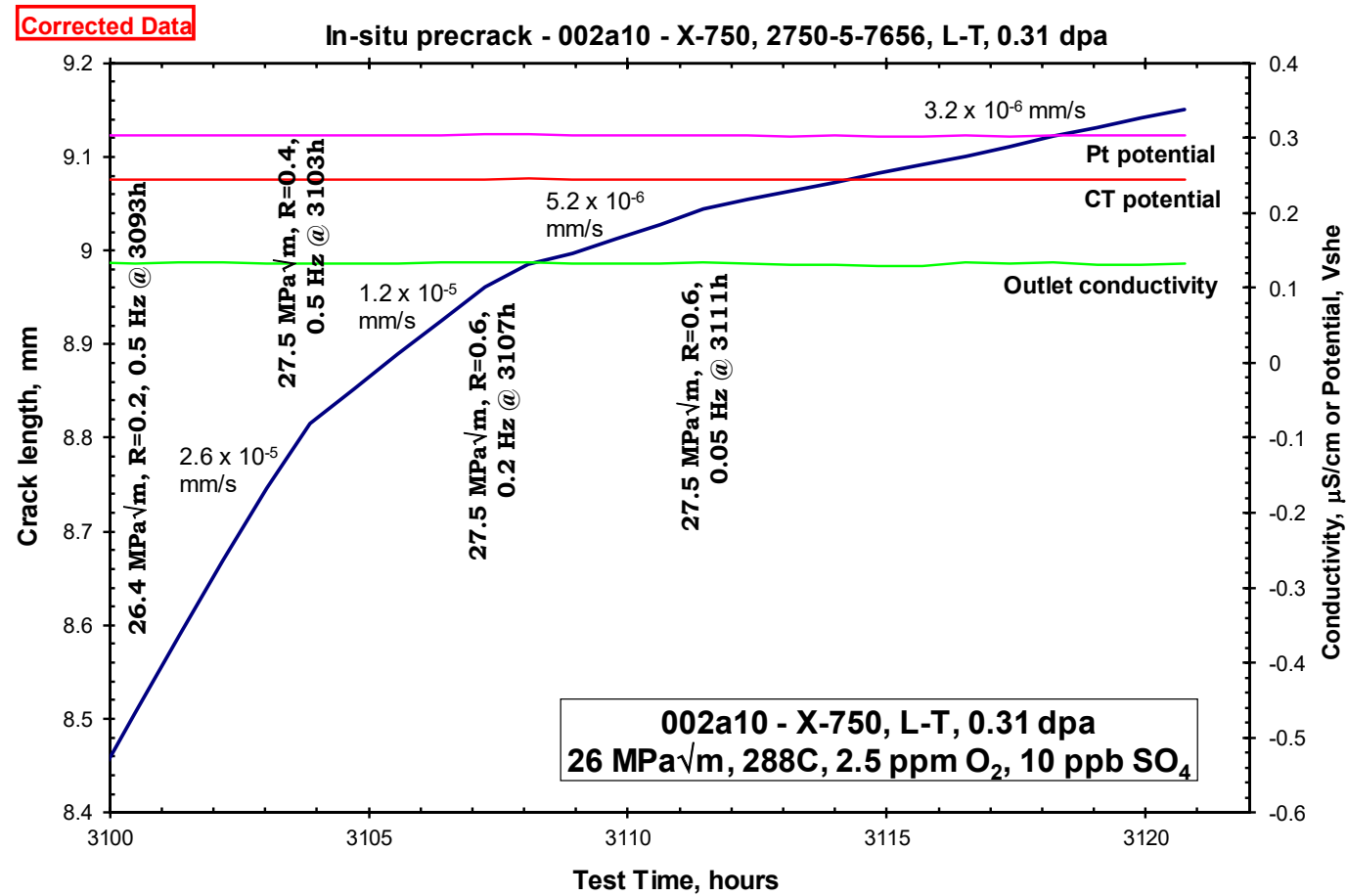

Figure 5-12: In-situ fatigue pre-cracking of specimen 10A0002A10 of alloy X-750 irradiated to 0.31 dpa.

After fatigue pre-cracking, intergranular transitioning was performed at loading ratio $\mathrm{R}=0.6$ and frequencies of 0.01 and $0.001 \mathrm{~Hz}$, with a 9,000s hold at $\mathrm{K}_{\max }$ (Figure 5-13). The change to constant $\mathrm{K}$ (no cycling) was made at 3,146 hours, and the subsequent response was well-behaved. The SCC growth rate at constant $\mathrm{K}$ was well-behaved at $2.6 \times 10^{-6} \mathrm{~mm} / \mathrm{s}$, essentially identical to the growth rate observed in the replicate specimen 10A0002A08 (Figure 5-3).

A change from $2.5 \mathrm{ppm} \mathrm{O}_{2}$ to $90 \mathrm{ppb}_{2}$ was made at 3,187 hours (Figure 5-14), resulting in a sudden drop in the potential of both the CT specimen and a Pt coupon. The change in growth rate was immediate, dropping to $3.6 \times 10^{-8} \mathrm{~mm} / \mathrm{s}$, representing a $\sim 72 \mathrm{X}$ reduction in growth rate. A return to $2.5 \mathrm{ppm} \mathrm{O}_{2}$ at 3,399 hours resulted in an increase in growth rate to $8 \times 10^{-7} \mathrm{~mm} / \mathrm{s}$, an increase of $22 \mathrm{X}$ over the growth rate in $90 \mathrm{ppb} \mathrm{H}_{2}$.

At 3,419 hours, gentle cycling was started (Figure 5-15), and - dK/da (falling K) was initiated to decrease from 29 to $18 \mathrm{MPa} \sqrt{\mathrm{m}}$, with the target $\mathrm{K}$ achieved at 3,444 hours. The step-wise growth of the crack results from each loading cycle, which occurs about every 3 hours $(1,000$ s cycle $+9,000$ s hold). The subsequent growth rate was well-behaved. At 3,523 hours, a change to $80 \mathrm{ppb} \mathrm{H}_{2}$ was made (Figure $5-16)$, and the corrosion potential and growth rate dropped rapidly, with a $68 \mathrm{X}$ reduction in growth rate. At 3,884 hours, there was a return to $2.5 \mathrm{ppm} \mathrm{O}_{2}$, and the growth rate transitioned close to its earlier rate in $2.5 \mathrm{ppm} \mathrm{O}_{2}$.

The $\mathrm{K}$ was then increased using gentle cycling and $+\mathrm{dK} / \mathrm{da}$ (rising $\mathrm{K}$ ) at 4,021 hours, and the target $\mathrm{K}$ of $30 \mathrm{MPa} \sqrt{\mathrm{m}}$ was achieved at 4,077 hours (Figure 5-17). The growth rate more than doubled from the increase in $\mathrm{K}$, consistent with a $\mathrm{K}$ dependency of about $\mathrm{K}^{1.5}$. At 4,120 hours, a change to $80 \mathrm{ppb}_{2}$ was made, and again the corrosion potential and growth rate suddenly dropped, reflecting a reduction of $58 \mathrm{X}$ (Figure 5-18).

Gentle cycling was introduced at 4,455 hours (Figure 5-18 and Figure 5-19), and there was an initially elevated growth rate that approached a steady-state asymptote. Linear extrapolation of the steadystate growth rate to the beginning of cycling indicates that the crack front unevenness was about $70 \mu \mathrm{m}-$ 
it is very likely that the initially higher growth rate results from regions of the crack front that lagged behind the average depth. A return to constant $\mathrm{K}$ occurred at 4,507 hours, and the growth rate returned to a similar value before cycling of $\sim 1 \times 10^{-8} \mathrm{~mm} / \mathrm{s}$. A change to $2.5 \mathrm{ppm} \mathrm{O}_{2}$ was made at 4,532 hours (Figure 5-18), and the growth rate increased to $5.8 \times 10^{-7} \mathrm{~mm} / \mathrm{s}$ - about half the growth rate observed at about 4,100 hours.

At 4,720 hours, gentle cycling and $+\mathrm{dK} / \mathrm{da}$ were used to increase from 30 to $48 \mathrm{MPa} \sqrt{\mathrm{m}}$ (Figure 5-20). The return to constant $\mathrm{K}$ (no cycling) conditions at 4,730 hours produced a stable growth rate of $6.5 \times 10^{-7} \mathrm{~mm} / \mathrm{s}$. At 4,792 hours, a change to $80 \mathrm{ppb} \mathrm{H}_{2}$ was made, and there was a rapid drop in corrosion potential and SCC growth rate (a 10X reduction).

The test was ended at 4,964 hours (Figure 5-21), and a macro photograph of the fracture surface is shown in Figure 5-22. The agreement with the DCPD was not unusual for irradiated specimens, with the average intergranular crack depth on the fracture surface being about $26 \%$ higher than was indicated by DCPD. The crack length, CGR, and $\mathrm{K}$ data were corrected in all plots. The post-test corrected $\mathrm{K}$ values were not much higher than intended during testing, because the error was not large and - equally important - the amount of total crack extension was not large. Table 5-2 is a summary of all the CGRs and related conditions under periods of constant applied $\mathrm{K}$ for this test.

Table 5-2: Constant K IASCC CGR and related conditions for specimen 10A0002A10 of alloy X750 irradiated to 0.31 dpa.

\begin{tabular}{|c|c|c|c|c|c|c|c|}
\hline $\begin{array}{c}\text { Test } \\
\text { Hours }\end{array}$ & $\begin{array}{c}\mathbf{K} \\
(\mathbf{M P a V m})\end{array}$ & Chemistry & Sulfate & $\begin{array}{c}\text { Outlet } \\
\text { Cond } \\
(\boldsymbol{\mu} \mathbf{S} / \mathbf{c m})\end{array}$ & $\begin{array}{c}\text { Time } \\
\text { Increment } \\
(\mathbf{h r})\end{array}$ & $\begin{array}{c}\text { Growth } \\
\text { Increment } \\
(\mathbf{m m})\end{array}$ & $\begin{array}{c}\text { Average } \\
\mathbf{C G R} \\
(\mathbf{m m} / \mathbf{s})\end{array}$ \\
\hline 3146 & 28 & NWC & 10 & 0.131 & 41 & 0.394 & $2.60 \mathrm{E}-06$ \\
\hline 3187 & 29 & HWC & 10 & 0.062 & 212 & 0.112 & $3.60 \mathrm{E}-08$ \\
\hline 3399 & 29 & NWC & 10 & 0.15 & 20 & 0.032 & $1.00 \mathrm{E}-06$ \\
\hline 3444 & 18 & NWC & 10 & 0.13 & 79 & 0.130 & $5.00 \mathrm{E}-07$ \\
\hline 3523 & 18 & HWC & 10 & 0.095 & 361 & 0.023 & $7.40 \mathrm{E}-09$ \\
\hline 3884 & 18 & NWC & 10 & 0.13 & 137 & 0.456 & $5.50 \mathrm{E}-07$ \\
\hline 4077 & 30 & NWC & 10 & 0.13 & 43 & 0.161 & $1.10 \mathrm{E}-06$ \\
\hline 4120 & 30 & HWC & 10 & 0.08 & 335 & 0.048 & $1.90 \mathrm{E}-08$ \\
\hline 4532 & 30 & NWC & 10 & 0.15 & 188 & 0.278 & $5.80 \mathrm{E}-07$ \\
\hline 4730 & 49 & NWC & 10 & 0.145 & 62 & 0.132 & $6.50 \mathrm{E}-07$ \\
\hline 4792 & 50 & HWC & 10 & 0.085 & 172 & 0.041 & $6.50 \mathrm{E}-08$ \\
\hline
\end{tabular}




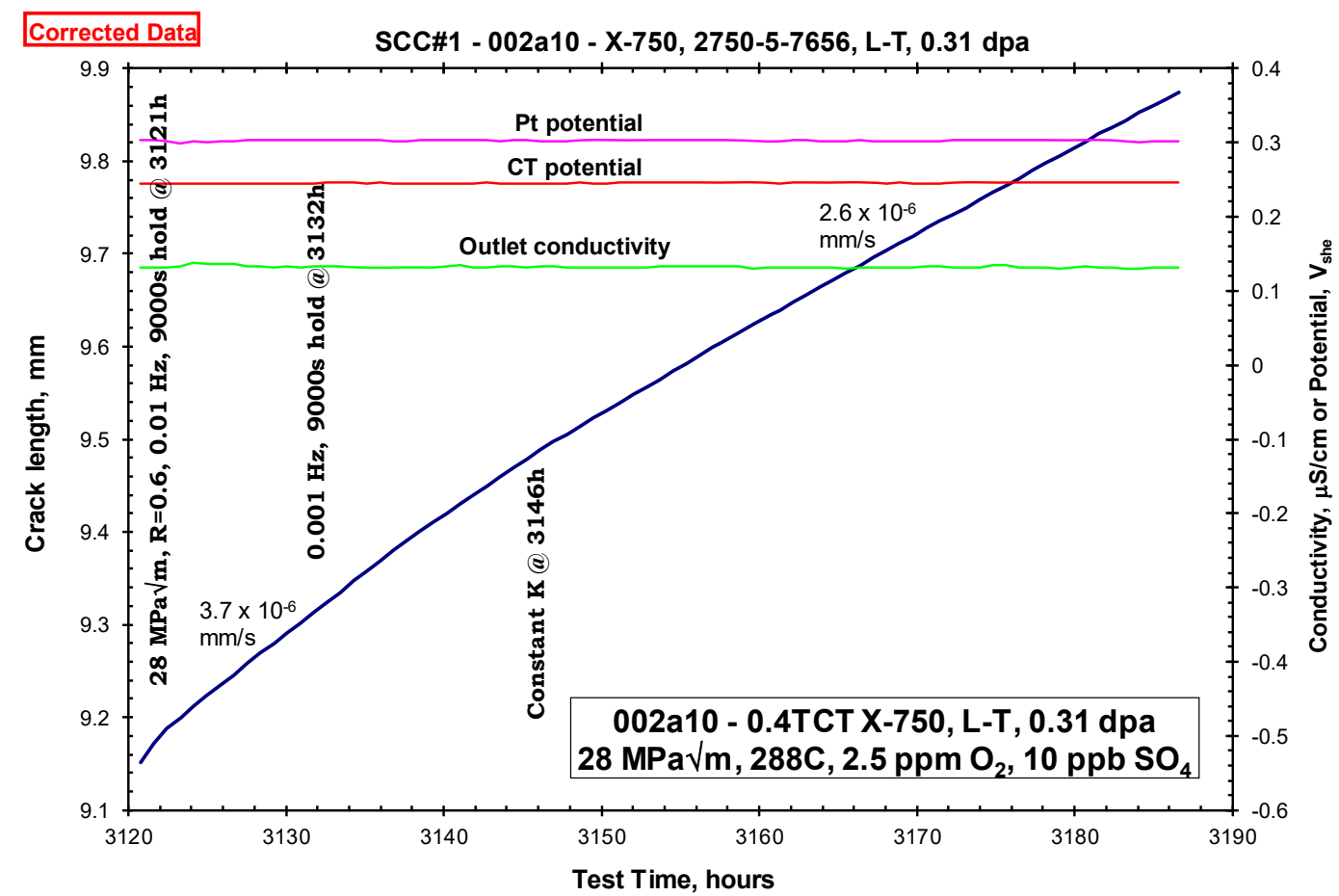

Figure 5-13: Transition to intergranular fracture and initial CGR under NWC for specimen 10A0002A10 of alloy X-750 irradiated to 0.31 dpa.

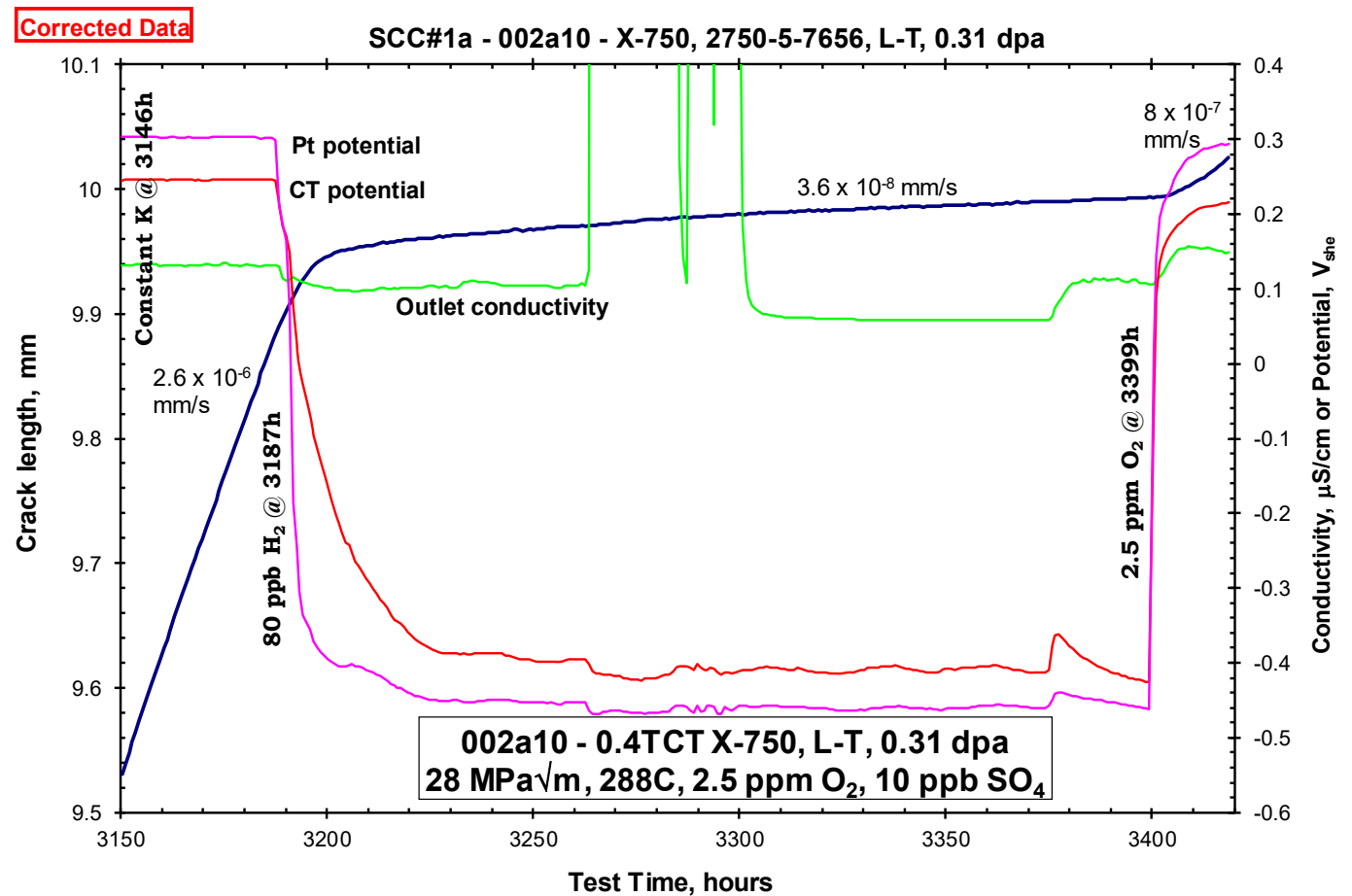

Figure 5-14: IASCC response for initial transition to HWC for specimen $10 \mathrm{A0002A10}$ of alloy $\mathrm{X}$ 750 irradiated to 0.31 dpa. 


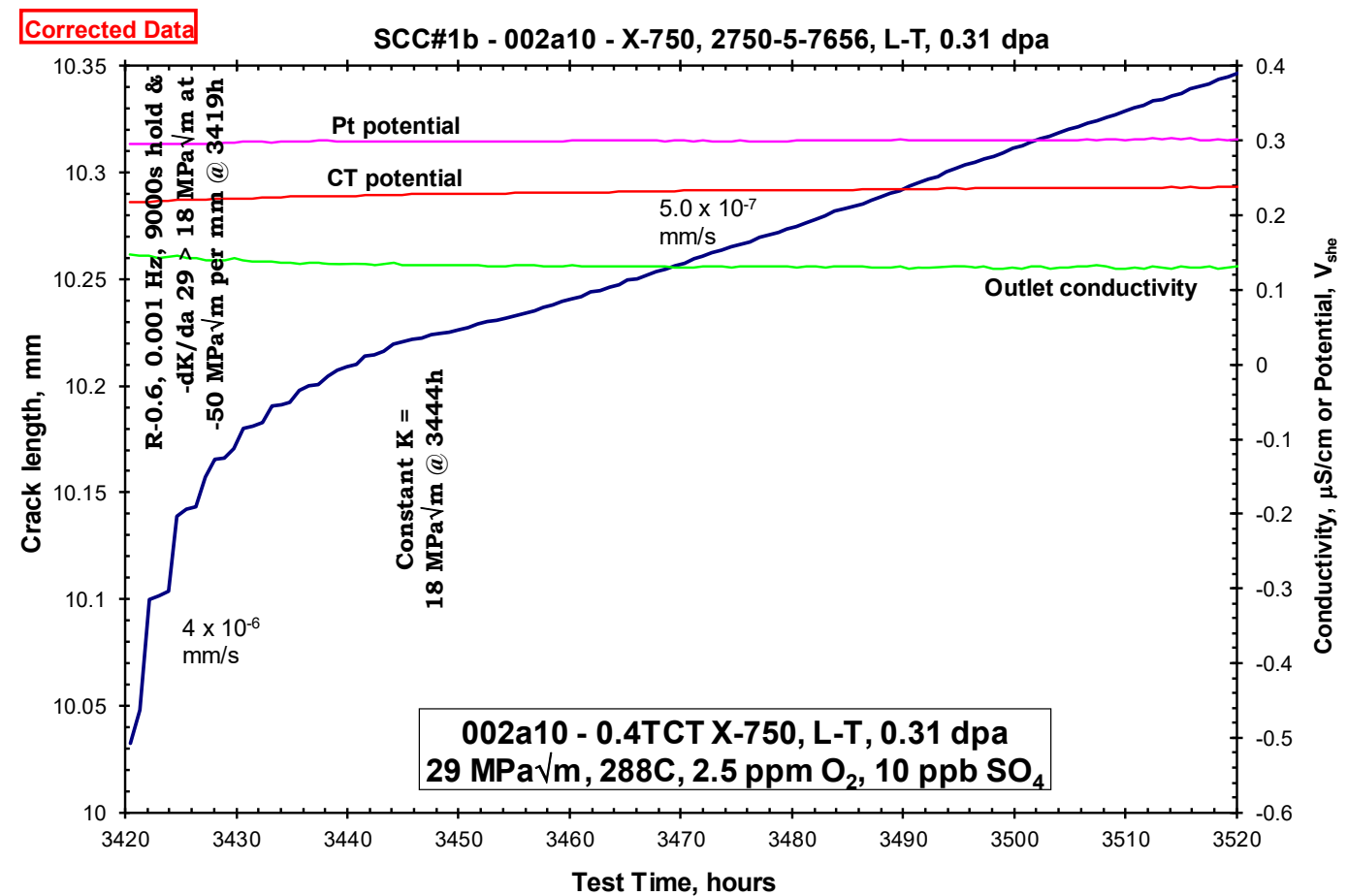

Figure 5-15: Effect of falling K (-dK/da) on NWC IASCC CGR for specimen 10A0002A10 of alloy $\mathrm{X}-750$ irradiated to 0.31 dpa.

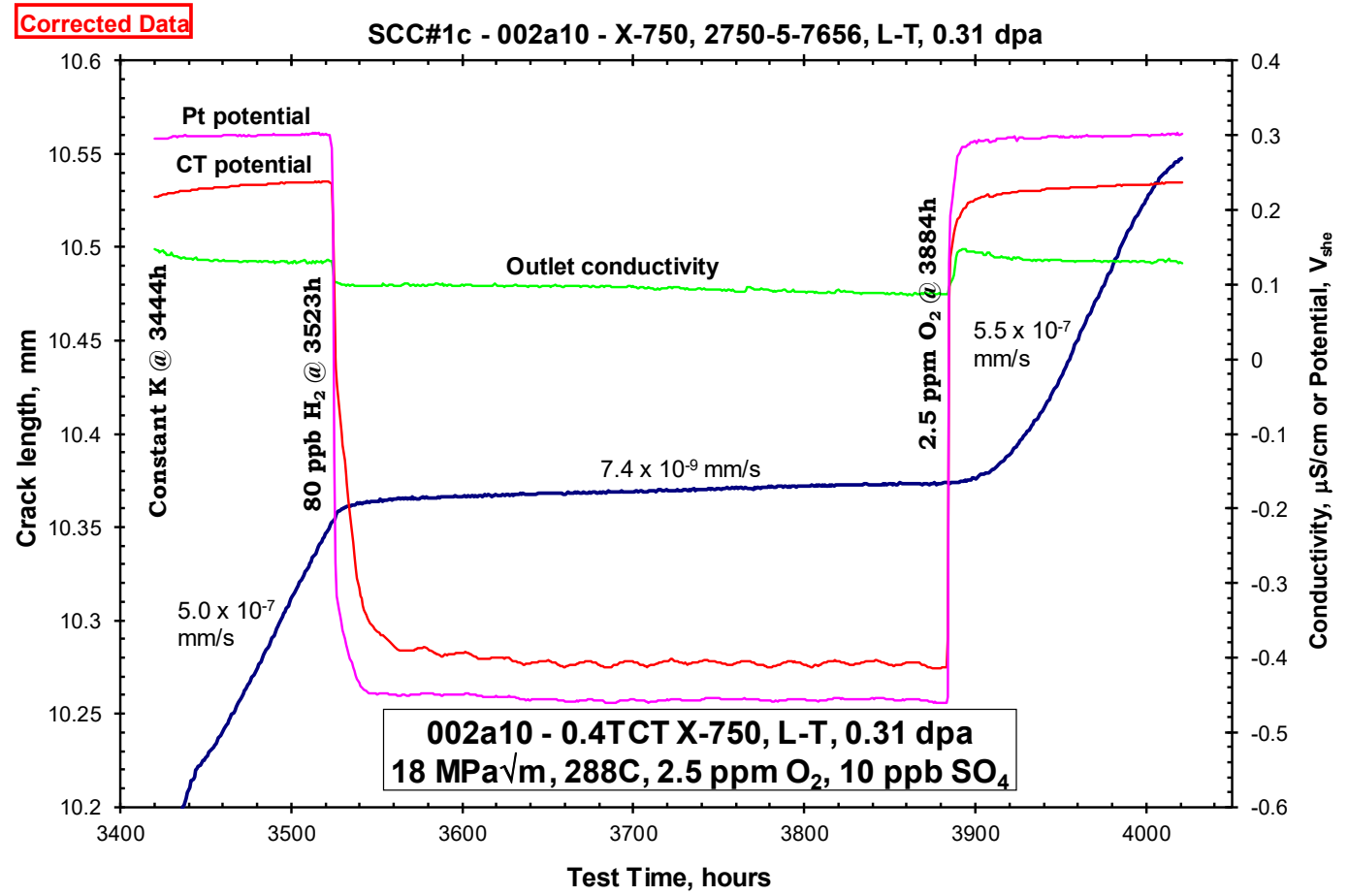

Figure 5-16: IASCC CGR under lower applied $\mathrm{K}$ and HWC for specimen 10A0002A10 of alloy X750 irradiated to 0.31 dpa. 


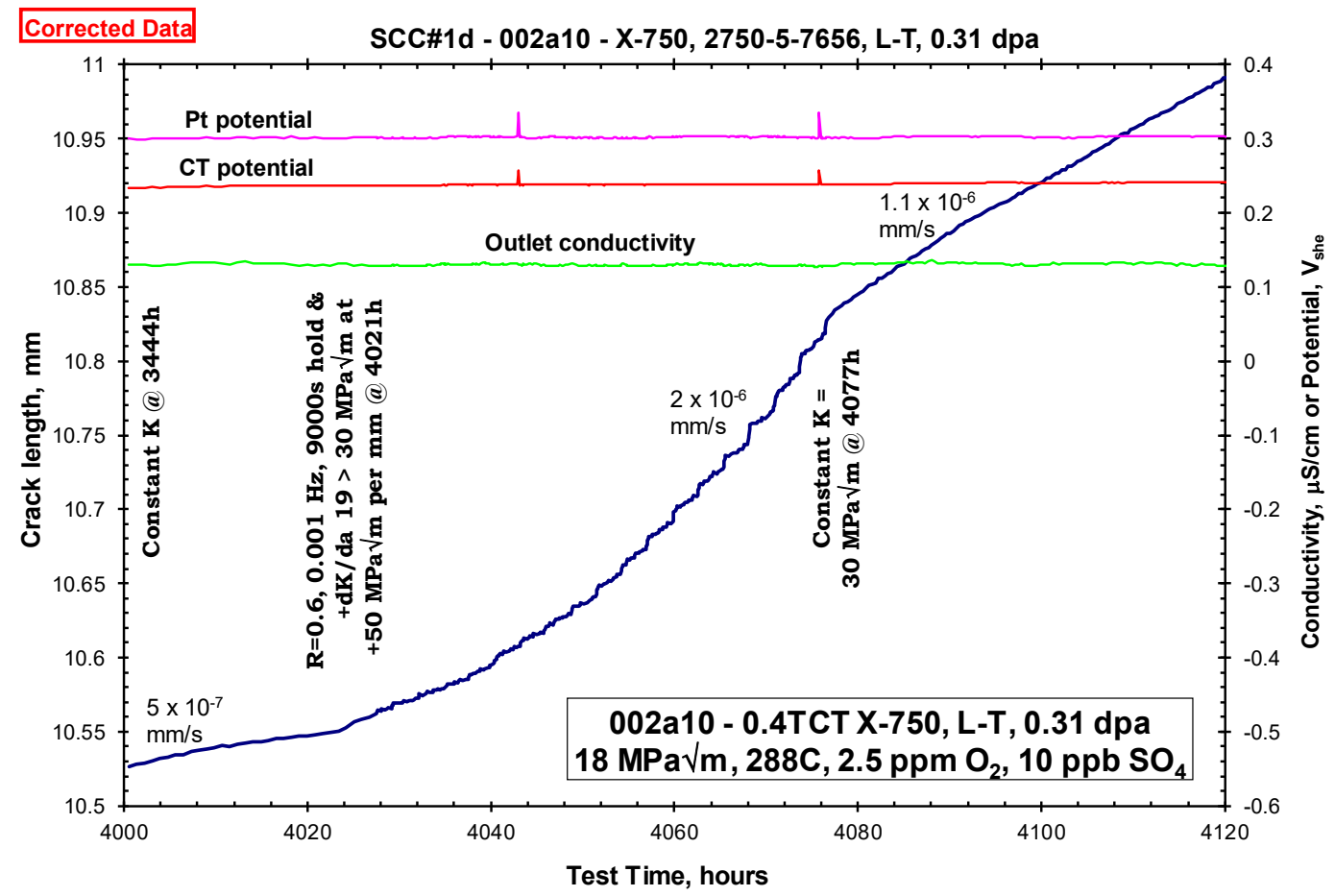

Figure 5-17: Effect of rising K $(+d K / d a)$ on NWC IASCC CGR for specimen 10A0002A10 of alloy $\mathrm{X}-750$ irradiated to 0.31 dpa.

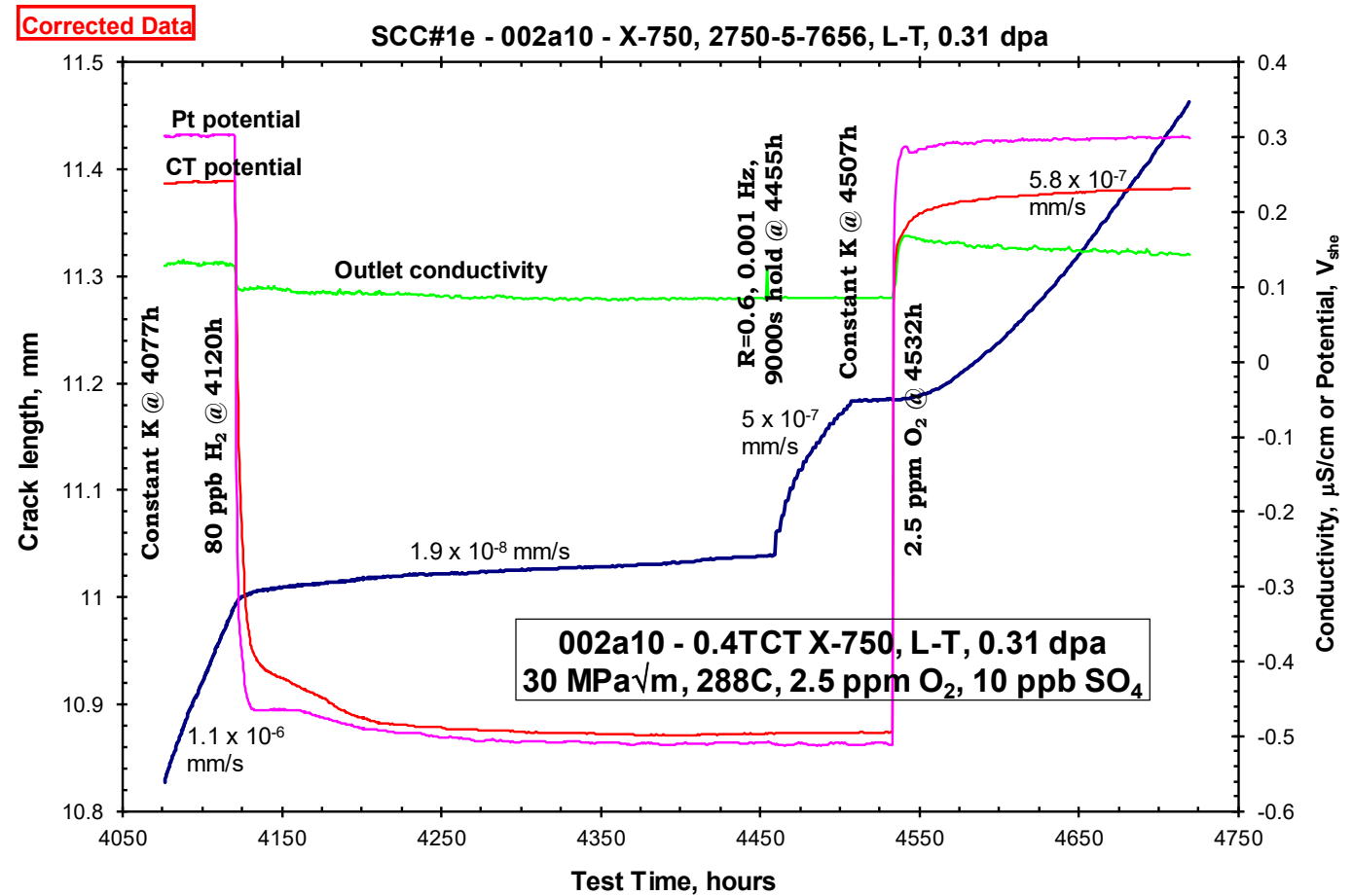

Figure 5-18: IASCC CGR following increased applied $K$ and in HWC for specimen 10A0002A10 of alloy $\mathrm{X}-750$ irradiated to 0.31 dpa. 


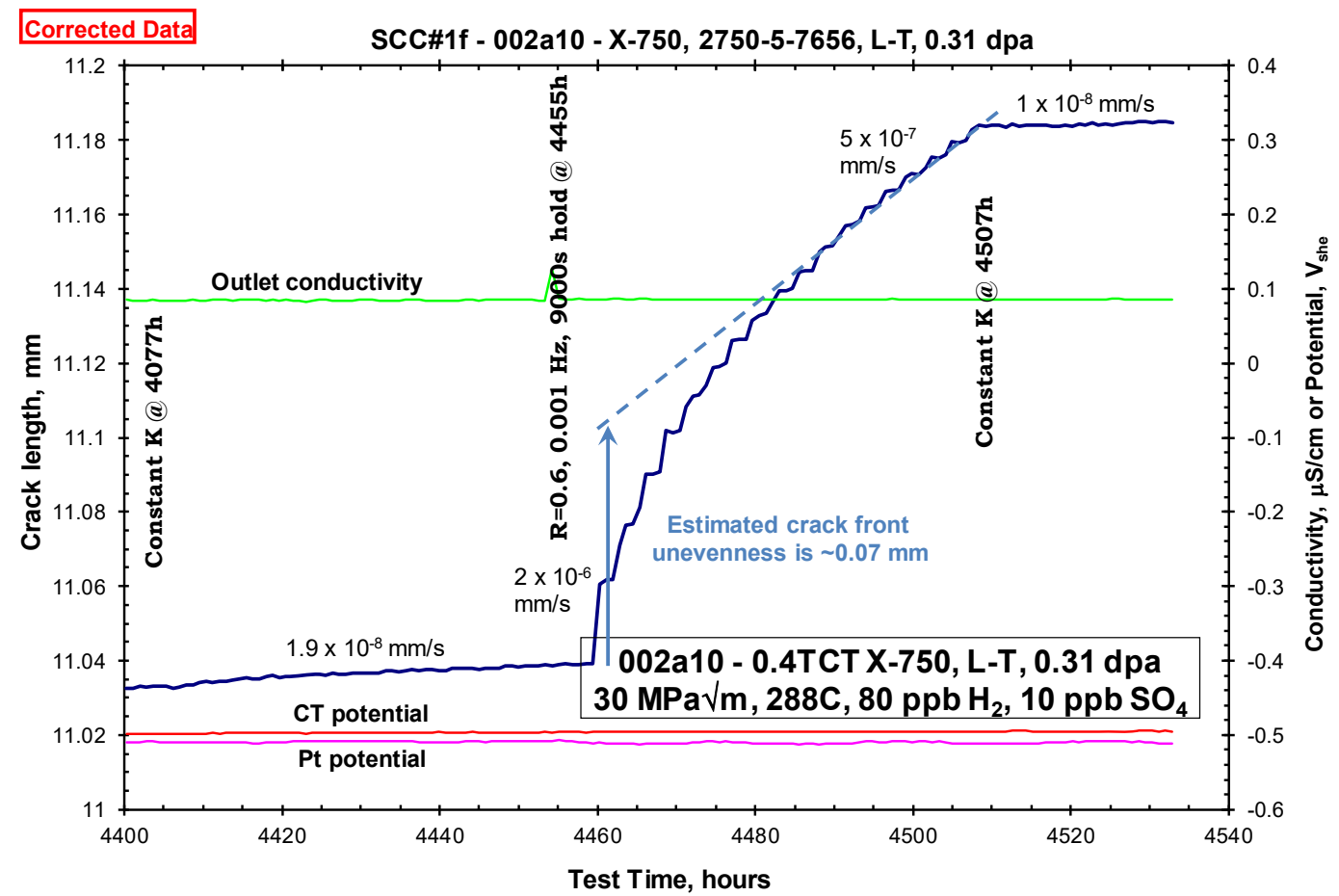

Figure 5-19: IASCC CGR response under gentle cycling to straighten the crack front for specimen 10A0002A10 of alloy $\mathrm{X}-750$ irradiated to 0.31 dpa.

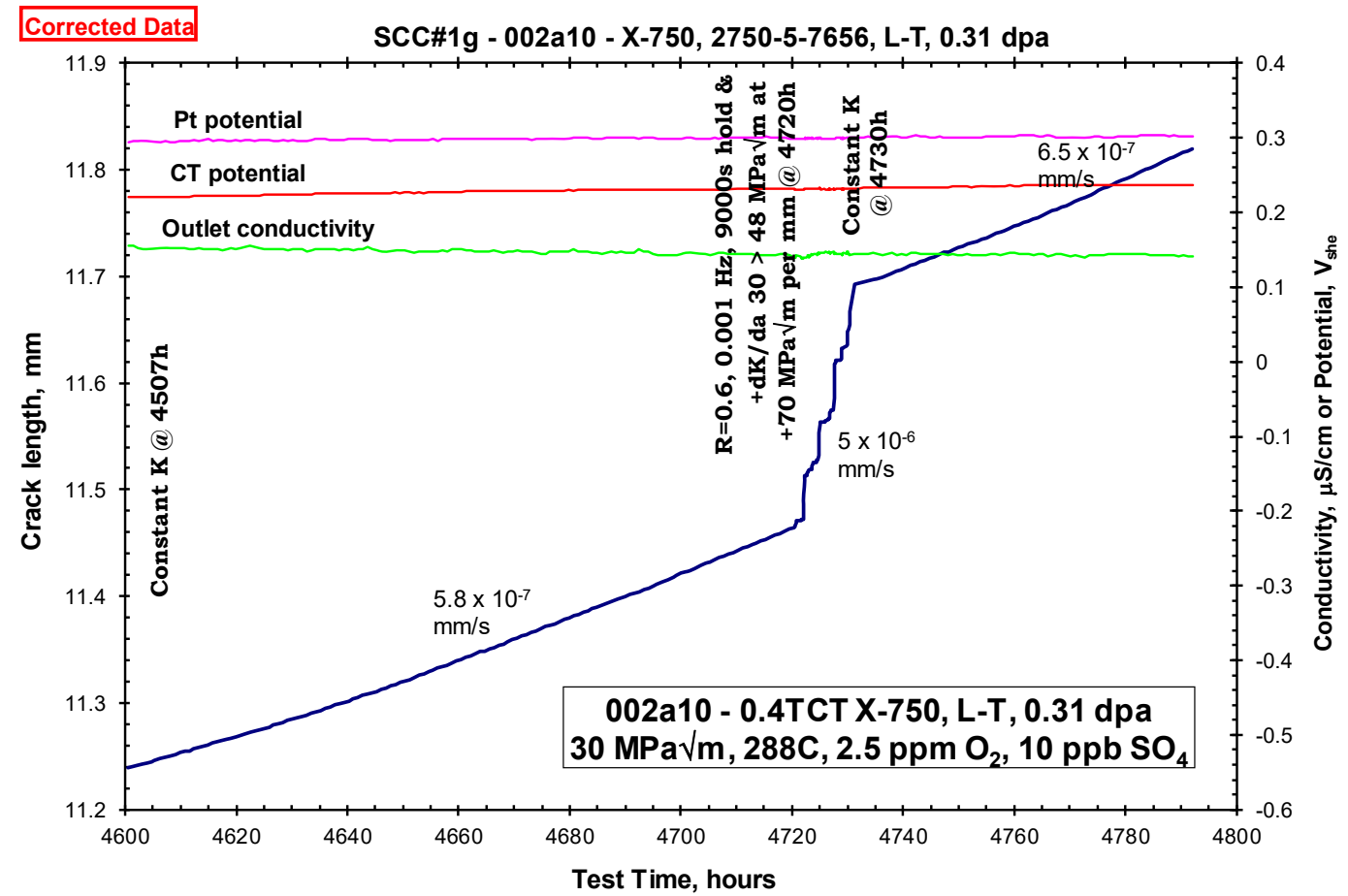

Figure 5-20: IASCC CGR response as $K$ is further increased for specimen 10A0002A10 of alloy $X$ 750 irradiated to 0.31 dpa. 


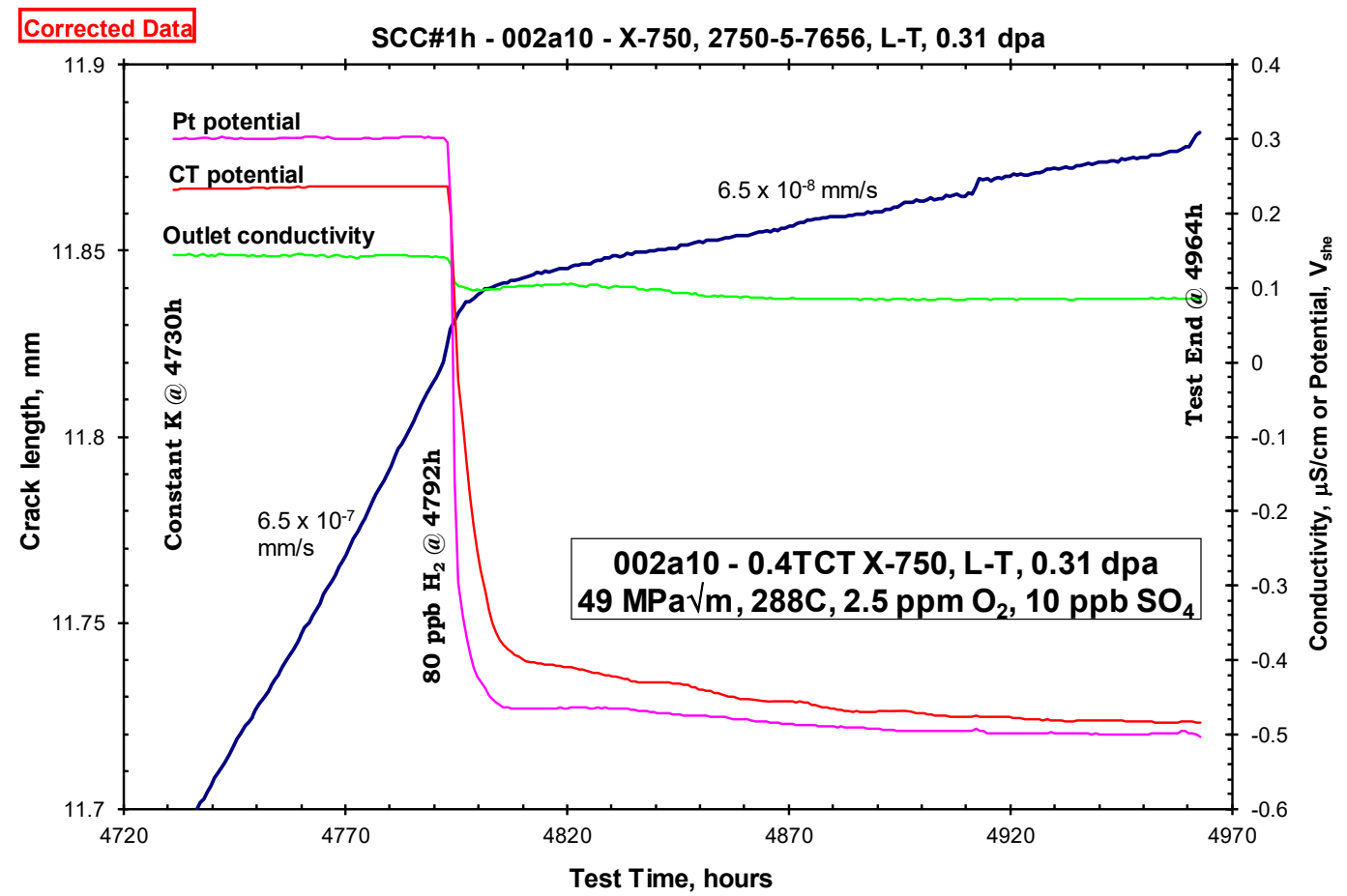

Figure 5-21: Final steps of IASCC test of specimen 10A0002A10 of alloy X-750 irradiated to 0.31 dpa.

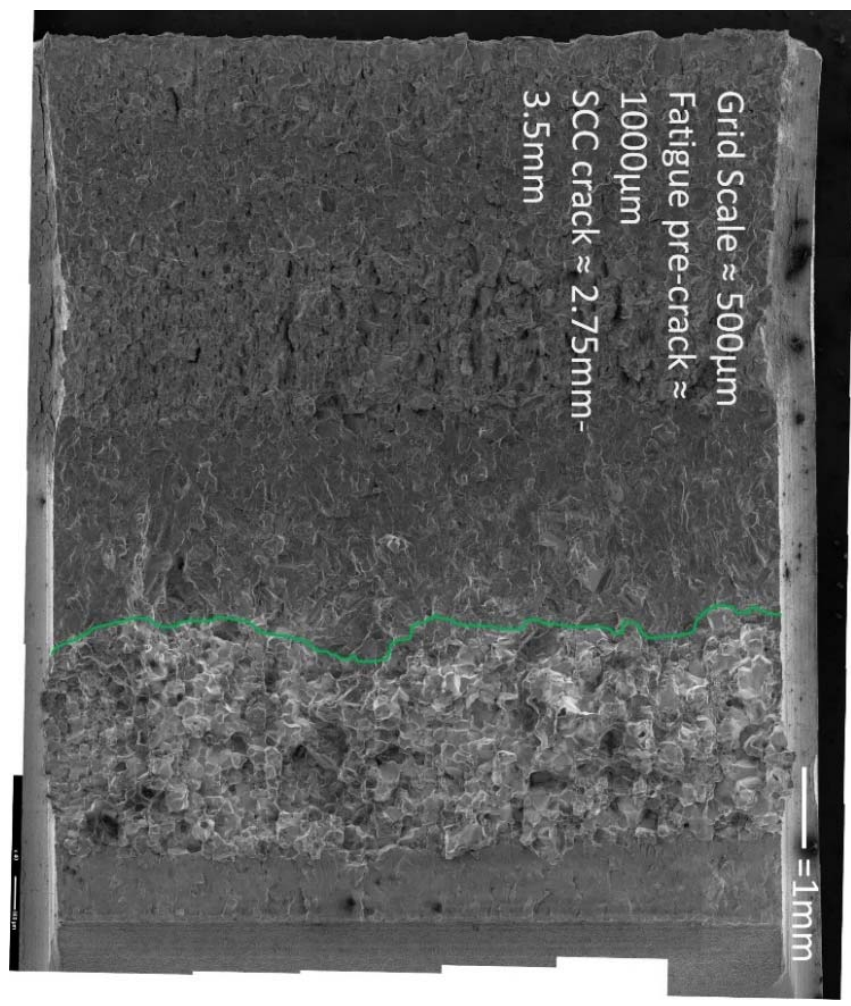

Figure 5-22: SEM micrograph showing the fracture surface of specimen $10 \mathrm{A0002} A 10$ of alloy $\mathrm{X}$ 750 irradiated to 0.31 dpa. 


\subsubsection{High Fluence Alloy X-750 IASCC Tests}

Specimens 10A0002B03 and 10A0002B09 were irradiated to fast-neutron damage levels of approximately 1.44 and $1.54 \mathrm{dpa}$, respectively, over two ATR cycles. Average irradiation temperatures for 10A0002B03 for the first and second cycle were estimated at $287^{\circ} \mathrm{C}$ and $257^{\circ} \mathrm{C}$, while maximum temperatures were estimated to be $295^{\circ} \mathrm{C}$ and $259^{\circ} \mathrm{C}$ for the first and second cycle, respectively. Average irradiation temperatures for specimen 10A0002B09 were estimated to be $335^{\circ} \mathrm{C}$ and $265^{\circ} \mathrm{C}$ for the first and second cycle, respectively, and maximums were estimated to be $347{ }^{\circ} \mathrm{C}$ and $268^{\circ} \mathrm{C}$. Details of these two IASCC tests follow; in all plots, the blue line tracks crack length vs. time (CGR).

\subsubsection{Specimen 10A0002B03 (1.44 dpa) alloy X-750 IASCC test}

The test was begun in $288^{\circ} \mathrm{C}$ pure water with $2.5 \mathrm{ppm} \mathrm{O}_{2}$. Figure 5-23 provides an overview of the response of specimen 10A0002B03, and Figure 5-24 shows the in-situ fatigue pre-cracking response. In each graph, the label in the outlined box represents the starting condition for that plot. Some graphs show additional $\mathrm{K}$ values, which represent an evolution in $\mathrm{K}$ resulting from the post-test correction, although some intentional changes in $\mathrm{K}$ were also made.

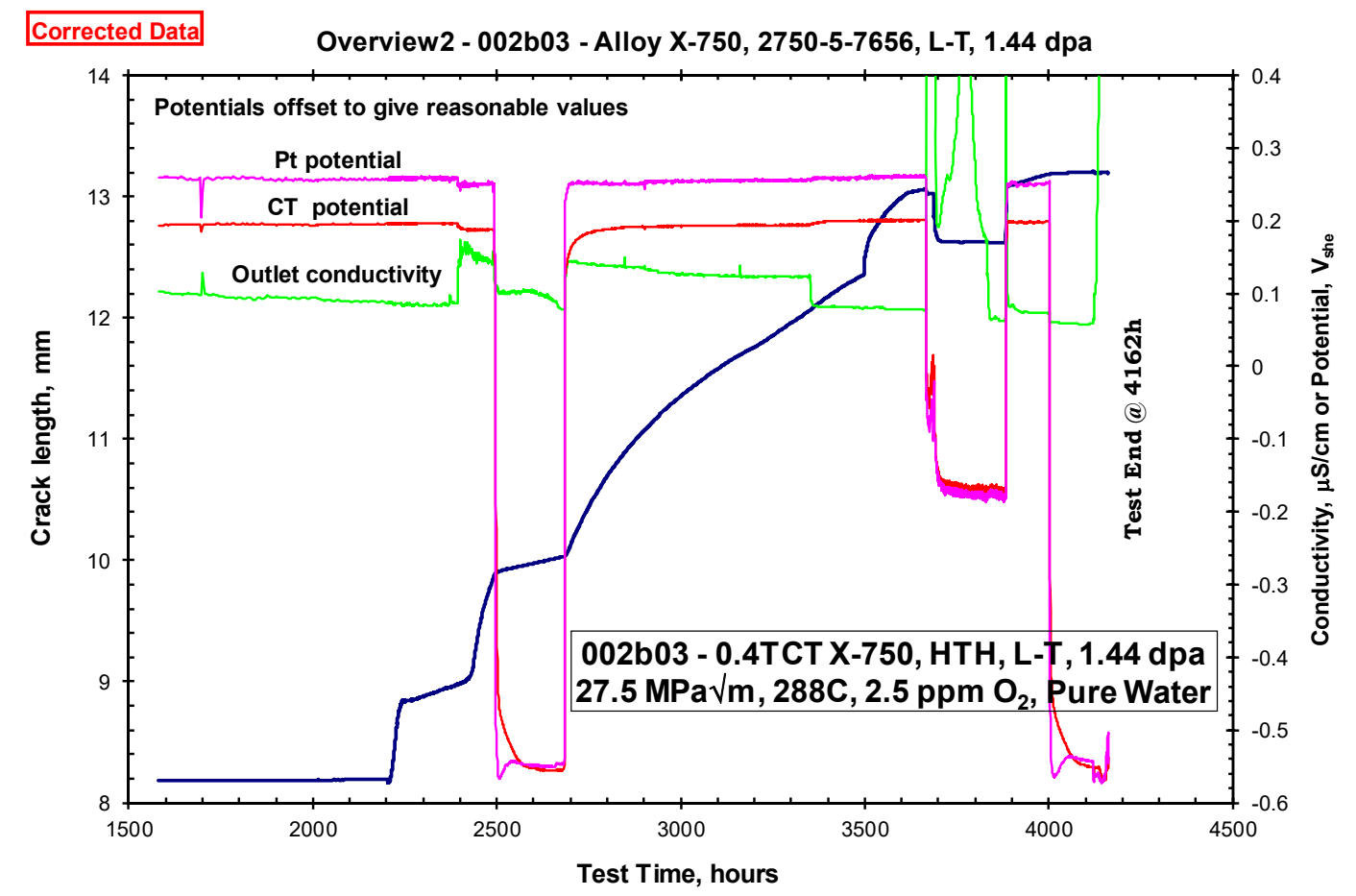

Figure 5-23: Overview of IASCC CGR test for specimen 10A0002B03 of alloy X-750 irradiated to 1.44 dpa. 


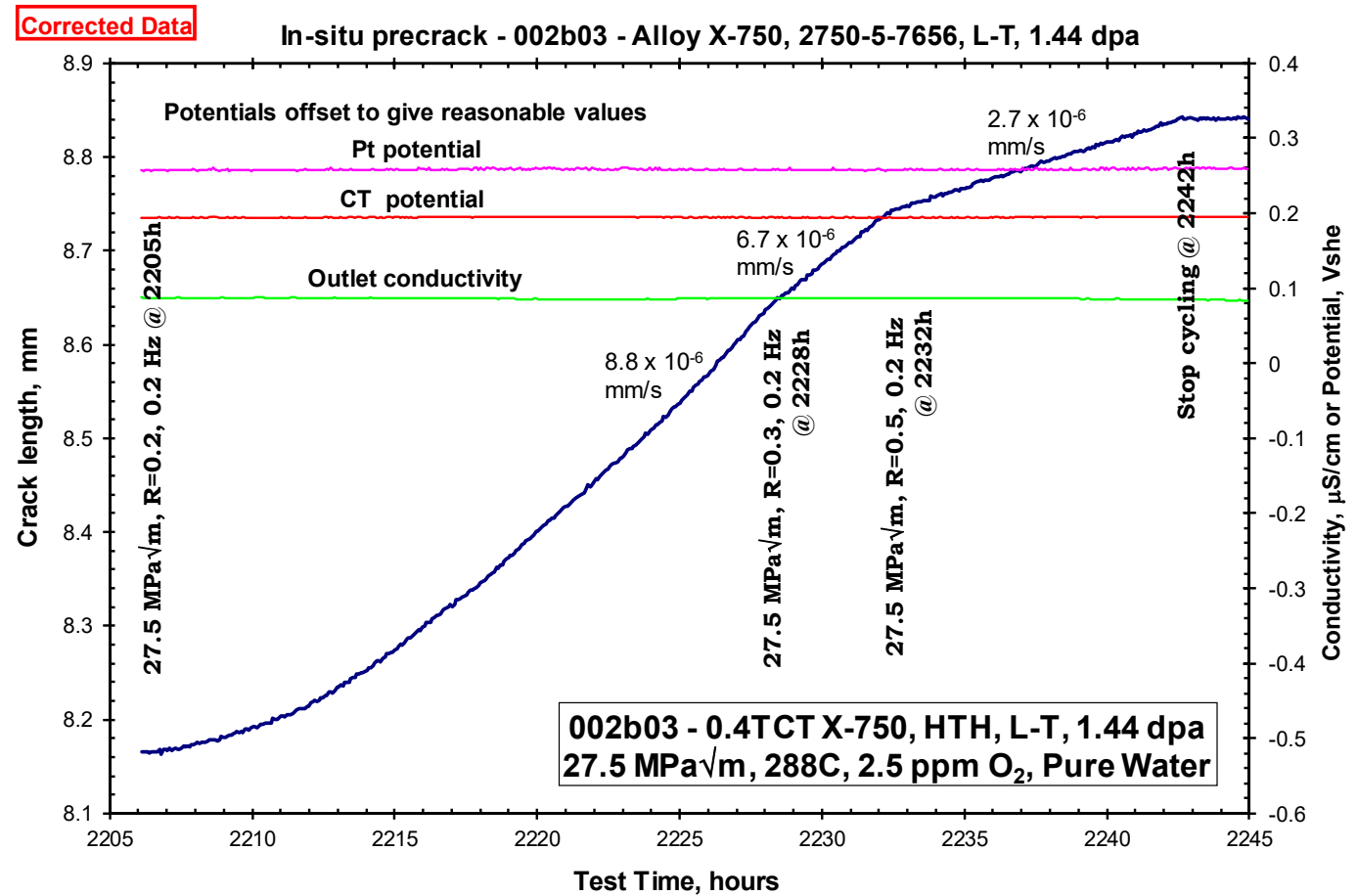

Figure 5-24: In-situ fatigue pre-cracking steps for specimen 10A0002B03 of alloy X-750 irradiated to 1.44 dpa.

After fatigue pre-cracking, intergranular transitioning was initiated at 2,277 hours at $\mathrm{K}_{\max }=27.5$ $\mathrm{MPa} \sqrt{\mathrm{m}}$, loading ratio $\mathrm{R}=0.6$, and frequencies of $0.01 \mathrm{~Hz}$ followed by $0.001 \mathrm{~Hz}$ (Figure 5-25). The difference in DCPD noise in Figure 5-25 is a result of changes in the amount of DCPD data averaged before saving. Injection of $8 \mathrm{ppb}$ sulfate $\left(\mathrm{as}_{2} \mathrm{SO}_{4}\right)$ was started at 2,392 hours. The CGR began to increase significantly, and a 3,500s hold time at $\mathrm{K}_{\max }$ was added at 2,436 hours (Figure 5-26), followed by hold times of 9,500s, 28,300s, and 85,900s. The change to constant $\mathrm{K}$ (no cycling) was made at 2,480 hours, and the subsequent response was well-behaved with a growth rate of $2.5 \times 10^{-6} \mathrm{~mm} / \mathrm{s}$.

A change from $2.5 \mathrm{ppm} \mathrm{O}_{2}$ to $95 \mathrm{ppb}_{2}$ was made at 2,492 hours (Figure 5-27), resulting in a sudden drop in the potential of both the CT specimen and a Pt coupon. The growth rate dropped to $1.9 \times 10^{-7}$ $\mathrm{mm} / \mathrm{s}-\mathrm{a} \sim 13 \mathrm{X}$ reduction. A return to $2.5 \mathrm{ppm} \mathrm{O}_{2}$ at 2,683 hours resulted in an increase in growth rate to $1.8 \times 10^{-6} \mathrm{~mm} / \mathrm{s}$, a $10 \mathrm{X}$ increase over the growth rate in $95 \mathrm{ppb} \mathrm{H}_{2}$.

The $\mathrm{K}$ was then decreased from $30.8 \mathrm{MPa} \sqrt{\mathrm{m}}$ using gentle cycling and $-\mathrm{dK} / \mathrm{da}$ (falling $\mathrm{K}$ ) at 2,753 hours, and the target $\mathrm{K}$ of $21.9 \mathrm{MPa} \sqrt{\mathrm{m}}$ was achieved at 3,174 hours (Figure 5-28 and Figure 5-29). Figure 5-29 shows the $\mathrm{K}$ trajectory during (what was intended to be) constant $\mathrm{K}$ conditions, as well as during $-\mathrm{dK} / \mathrm{da}$. The growth rate decreased by about $3.5 \mathrm{X}$, consistent with a $\mathrm{K}$ dependency of about $\mathrm{K}^{3.6}$. At 3,351 hours, sulfate injection was stopped, and the subsequent growth rate changed very little.

Gentle cycling was introduced at 3,497 hours (Figure 5-30 and Figure 5-31), and there was an initial elevated growth rate that approached a steady-state asymptote. Linear extrapolation of the steady-state growth rate to the beginning of cycling indicates that the crack front unevenness was about $340 \mu \mathrm{m}$ - it is very likely that the initially higher growth rate results from regions of the crack front that lagged behind the average depth. A return to constant $\mathrm{K}$ occurred at 3,618 hours, and the growth rate dropped to $1.6 \mathrm{x}$ $10^{-7} \mathrm{~mm} / \mathrm{s}$. There was a period of time during which the test was cooled, then re-heated and re-loaded (Figure 5-31 and Figure 5-32), after which the growth rate was somewhat higher at $2.6 \times 10^{-7} \mathrm{~mm} / \mathrm{s}$.

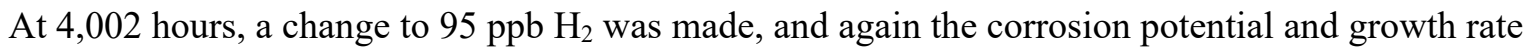
suddenly dropped, reflecting a 7X reduction in growth rate (Figure 5-31 and Figure 5-32). The test ended 
at 4,162 hours (Figure 5-33), and a macro photograph of the fracture surface is shown in Figure 5-34. The average intergranular crack depth on the fracture surface is about $59 \%$ higher than was indicated by DCPD. The crack length, CGR, and K data were corrected for all plots. The post-test corrected K values were higher than intended during testing because the error was moderately large and - equally important - the amount of total crack extension was moderately large. Table 5-3 is a summary of all the CGRs and related conditions under periods of constant applied $\mathrm{K}$ for this test.

Table 5-3: Constant K IASCC CGR and related conditions for specimen $10 \mathrm{A0002B03}$ of alloy $\mathrm{X}$ 750 irradiated to $1.44 \mathrm{dpa}$; blue highlights indicate higher confidence data.

\begin{tabular}{|c|c|c|c|c|c|c|c|}
\hline $\begin{array}{c}\text { Test } \\
\text { Hours }\end{array}$ & $\begin{array}{c}\mathbf{K} \\
(\mathbf{M P a V m})\end{array}$ & Chemistry & Sulfate & $\begin{array}{c}\text { Outlet } \\
\text { Cond } \\
(\boldsymbol{\mu} \mathbf{S} / \mathbf{c m})\end{array}$ & $\begin{array}{c}\text { Time } \\
\text { Increment } \\
(\mathbf{h r})\end{array}$ & $\begin{array}{c}\text { Growth } \\
\text { Increment } \\
(\mathbf{m m})\end{array}$ & $\begin{array}{c}\text { Average } \\
\text { CGR } \\
(\mathbf{m m} / \mathbf{s})\end{array}$ \\
\hline 2467 & 29 & NWC & 8 & 0.148 & 25 & 0.232 & $2.50 \mathrm{E}-06$ \\
\hline 2492 & 30 & HWC & 8 & 0.1 & 191 & 0.160 & $1.90 \mathrm{E}-07$ \\
\hline 2683 & 31 & NWC & 8 & 0.141 & 70 & 0.428 & $1.80 \mathrm{E}-06$ \\
\hline 3174 & 22 & NWC & 8 & 0.123 & 177 & 0.346 & $5.20 \mathrm{E}-07$ \\
\hline 3351 & 23 & NWC & 0 & 0.082 & 146 & 0.291 & $4.50 \mathrm{E}-07$ \\
\hline 3887 & 24 & NWC & 0 & 0.073 & 115 & 0.104 & $2.60 \mathrm{E}-07$ \\
\hline 4002 & 26 & HWC & 0 & 0.06 & 151 & 0.013 & $3.60 \mathrm{E}-08$ \\
\hline
\end{tabular}

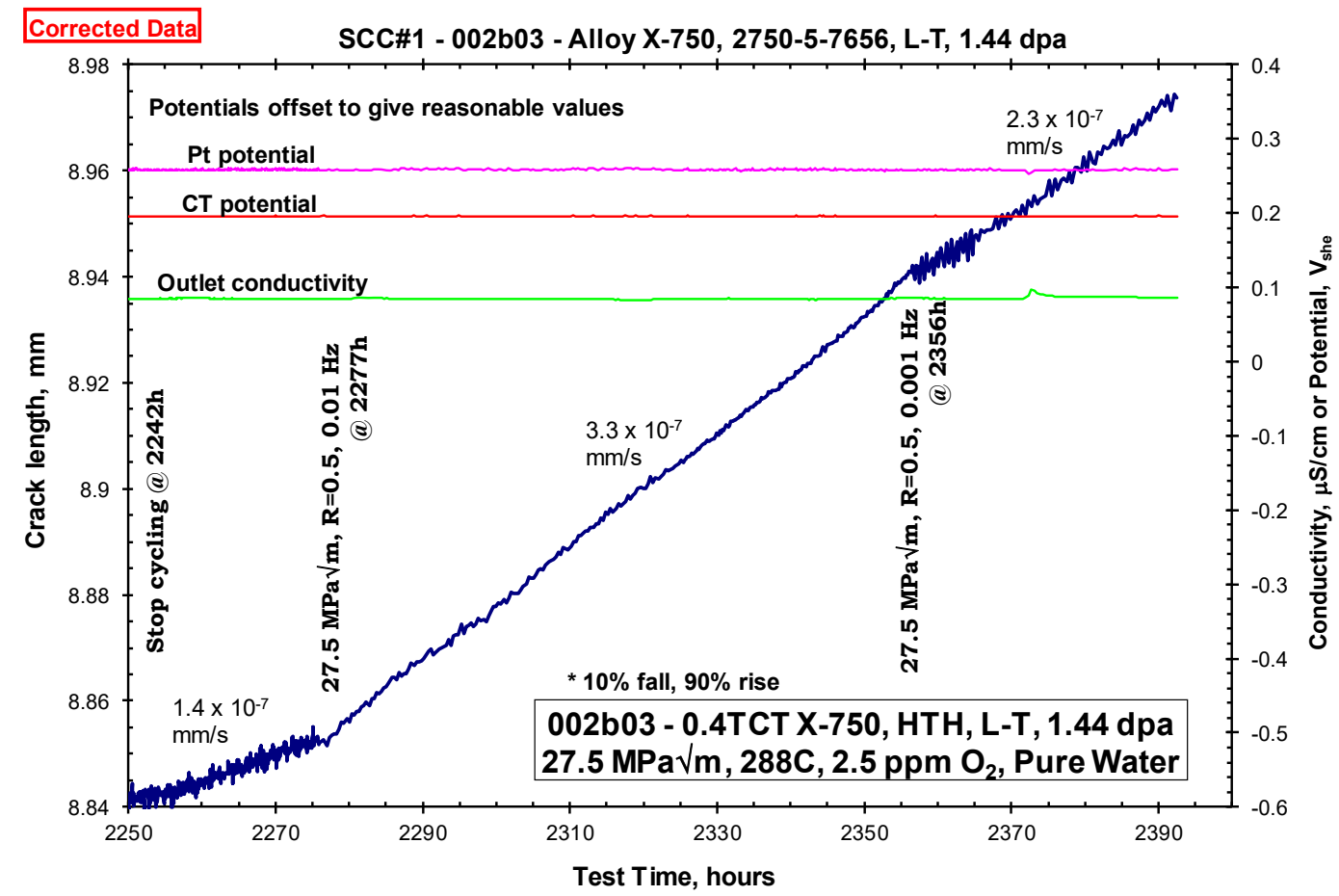

Figure 5-25: IASCC test transition to intergranular fracture for specimen 10A0002B03 of alloy $\mathrm{X}$ 750 irradiated to 1.44 dpa. 


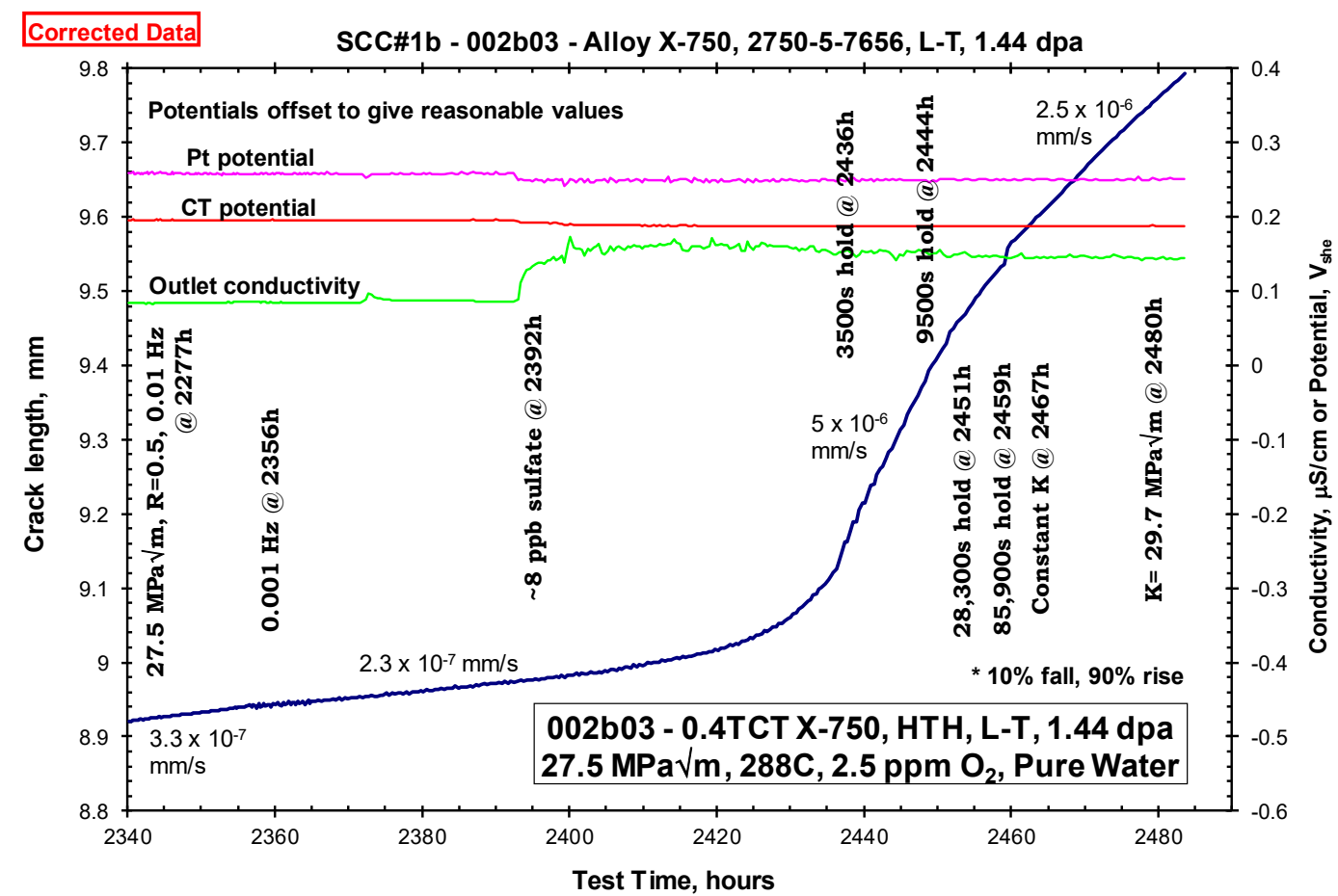

Figure 5-26: Final transition to intergranular fracture and initial constant $K$ for specimen $10 \mathrm{~A} 0002 \mathrm{B03}$ of alloy $\mathrm{X}-750$ irradiated to $1.44 \mathrm{dpa}$.

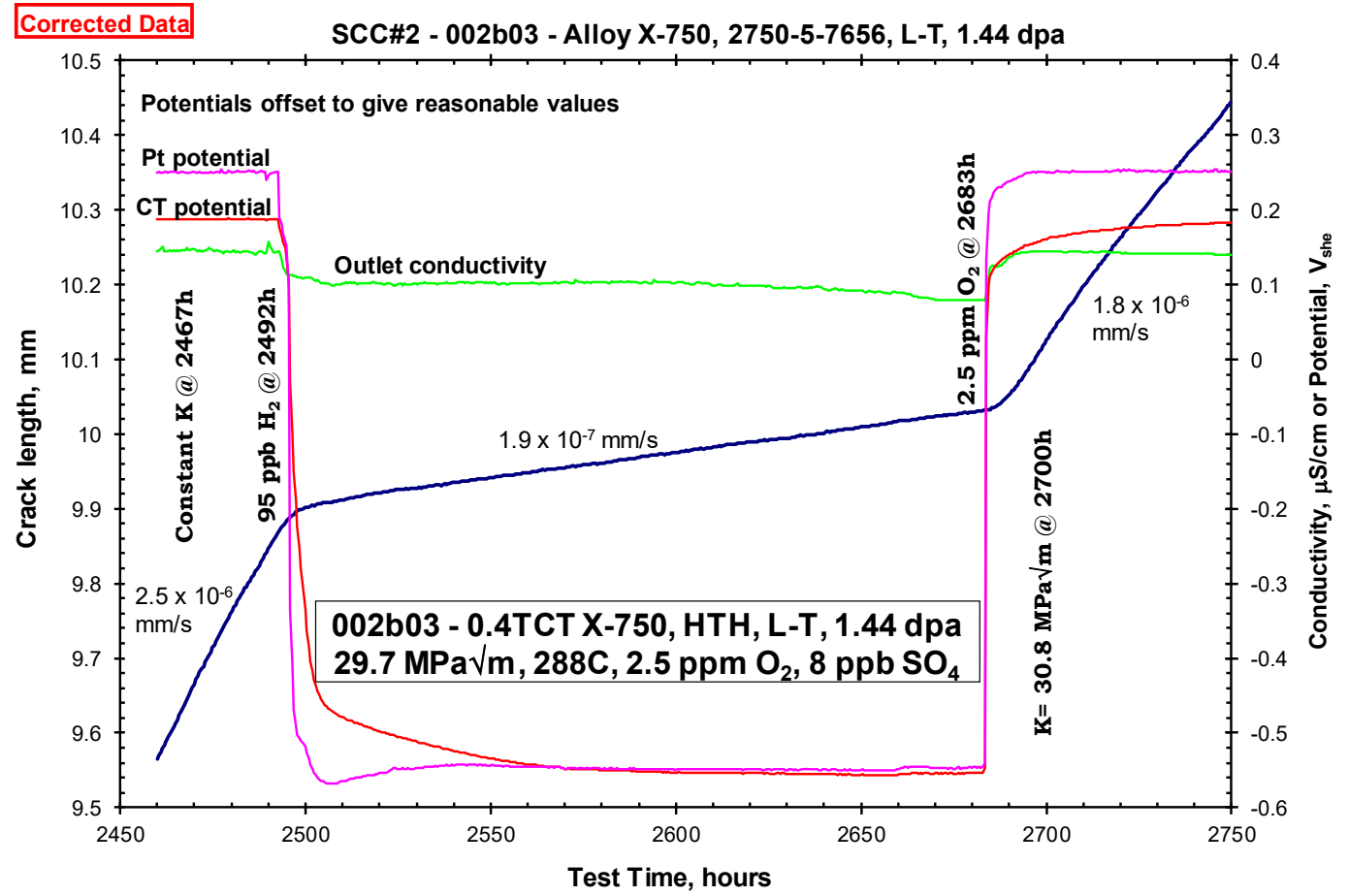

Figure 5-27: First transition from NWC to HWC in IASCC test for specimen 10A0002B03 of alloy X-750 irradiated to 1.44 dpa. 


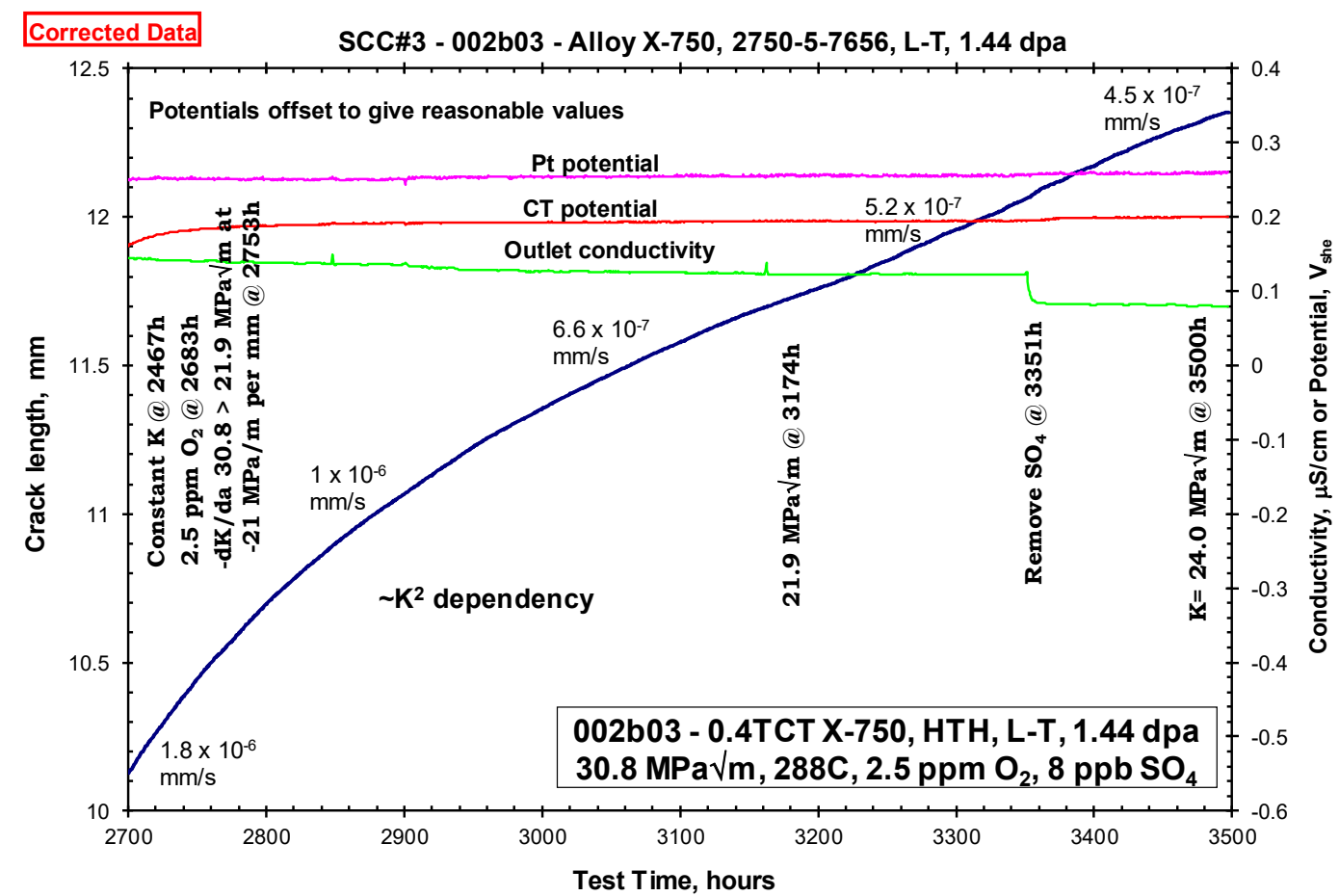

Figure 5-28: IASCC CGR test with -dK/da under NWC for specimen 10A0002B03 of alloy X-750 irradiated to 1.44 dpa.

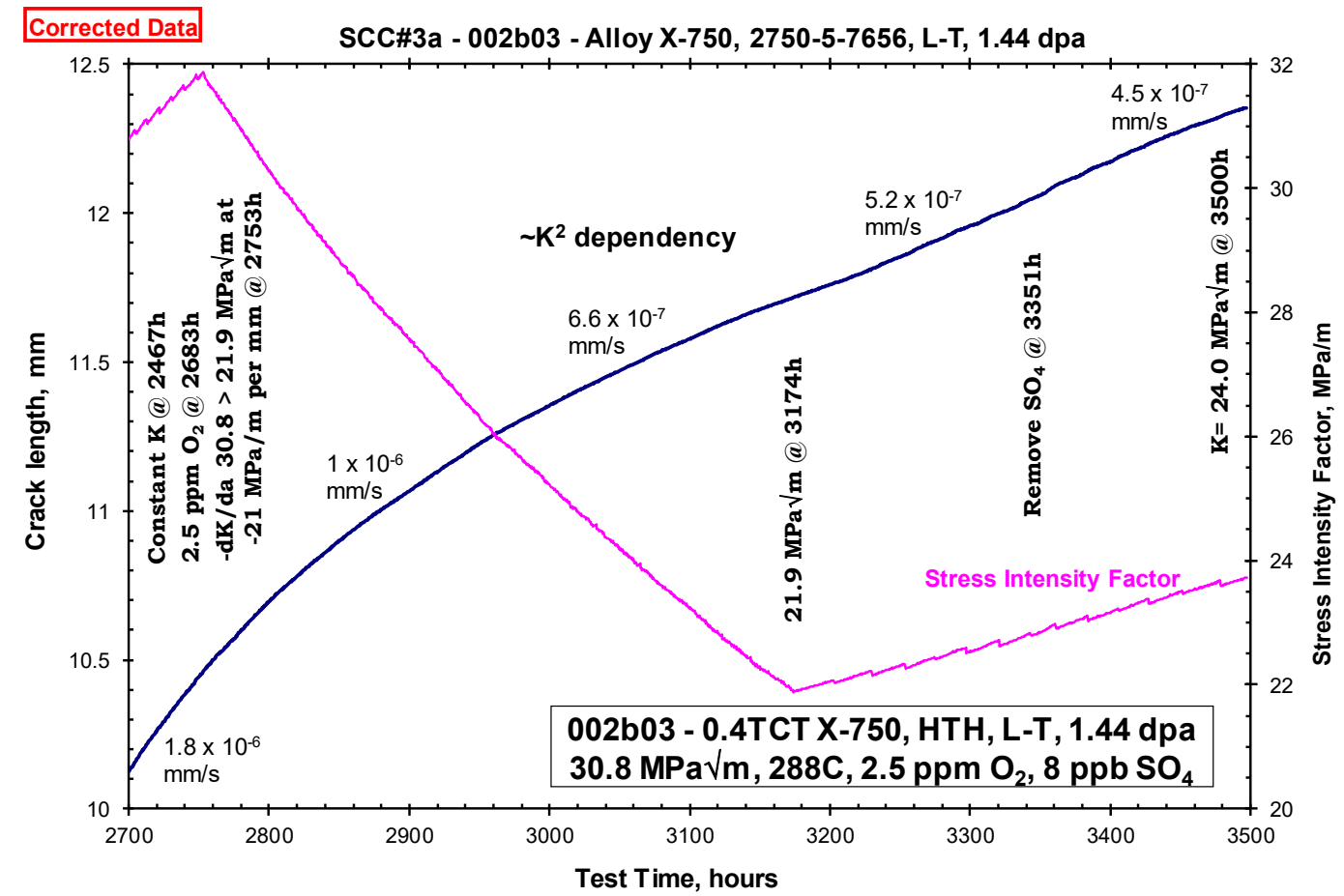

Figure 5-29: Applied K trajectory overlaid on IASCC CGR plot under -dK/da for specimen $10 \mathrm{A0002} B 03$ of alloy $\mathrm{X}-750$ irradiated to $1.44 \mathrm{dpa}$. 


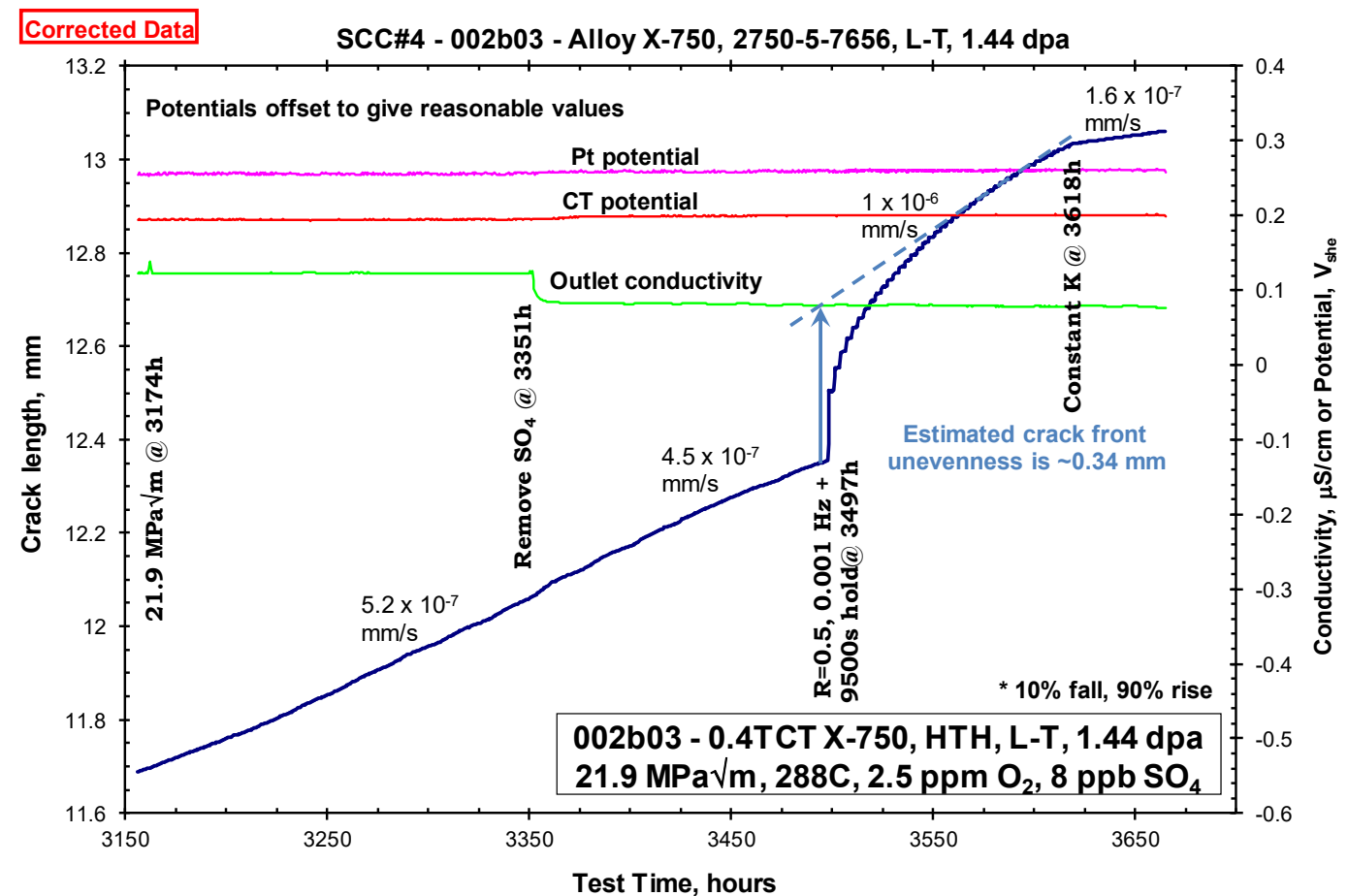

Figure 5-30: Crack front straightening during IASCC CGR test of specimen 10A0002B03 of alloy X-750 irradiated to 1.44 dpa.

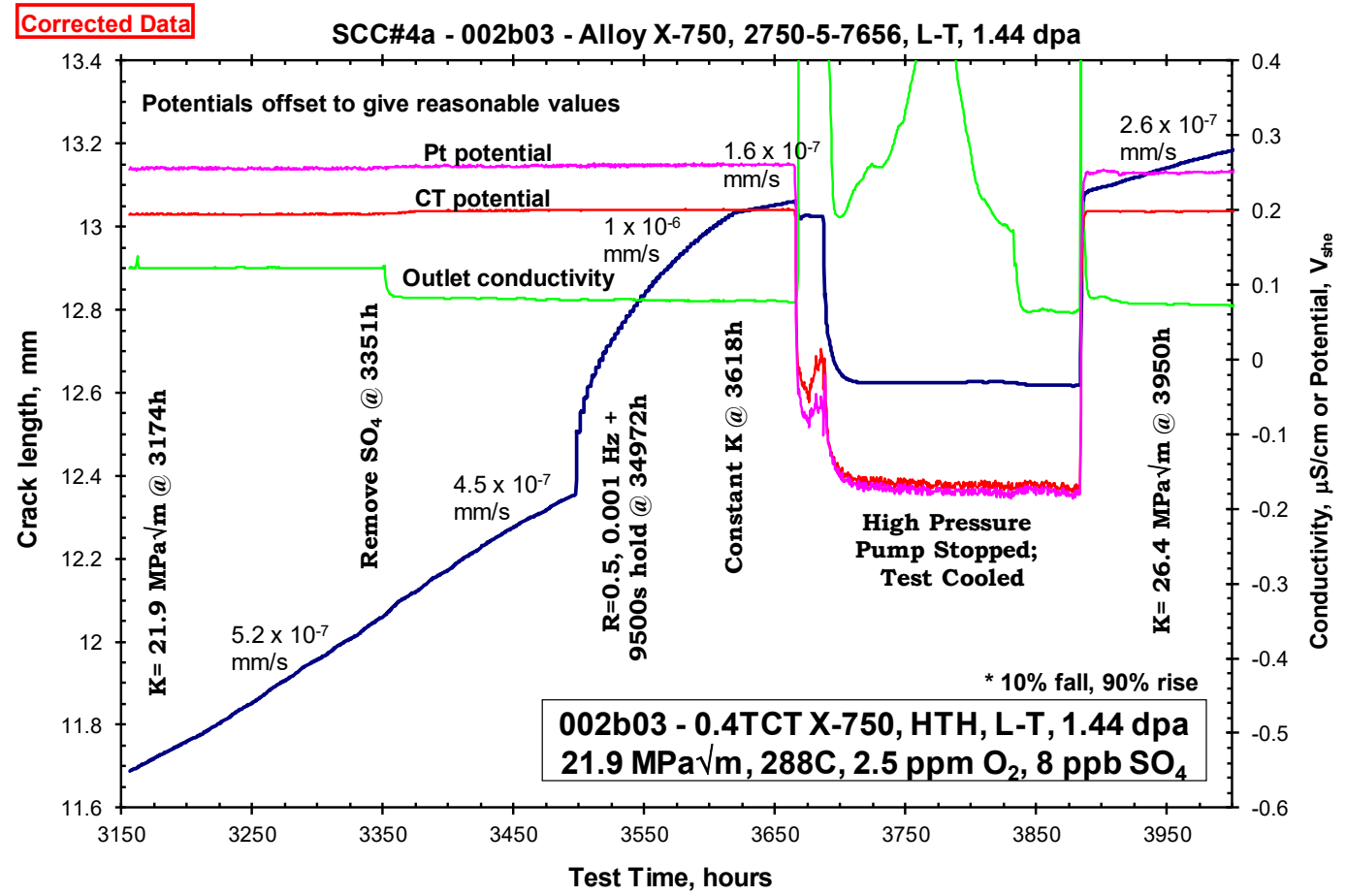

Figure 5-31: IASCC CGR during high pressure pump malfunction for specimen 10A0002B03 of alloy X-750 irradiated to 1.44 dpa. 


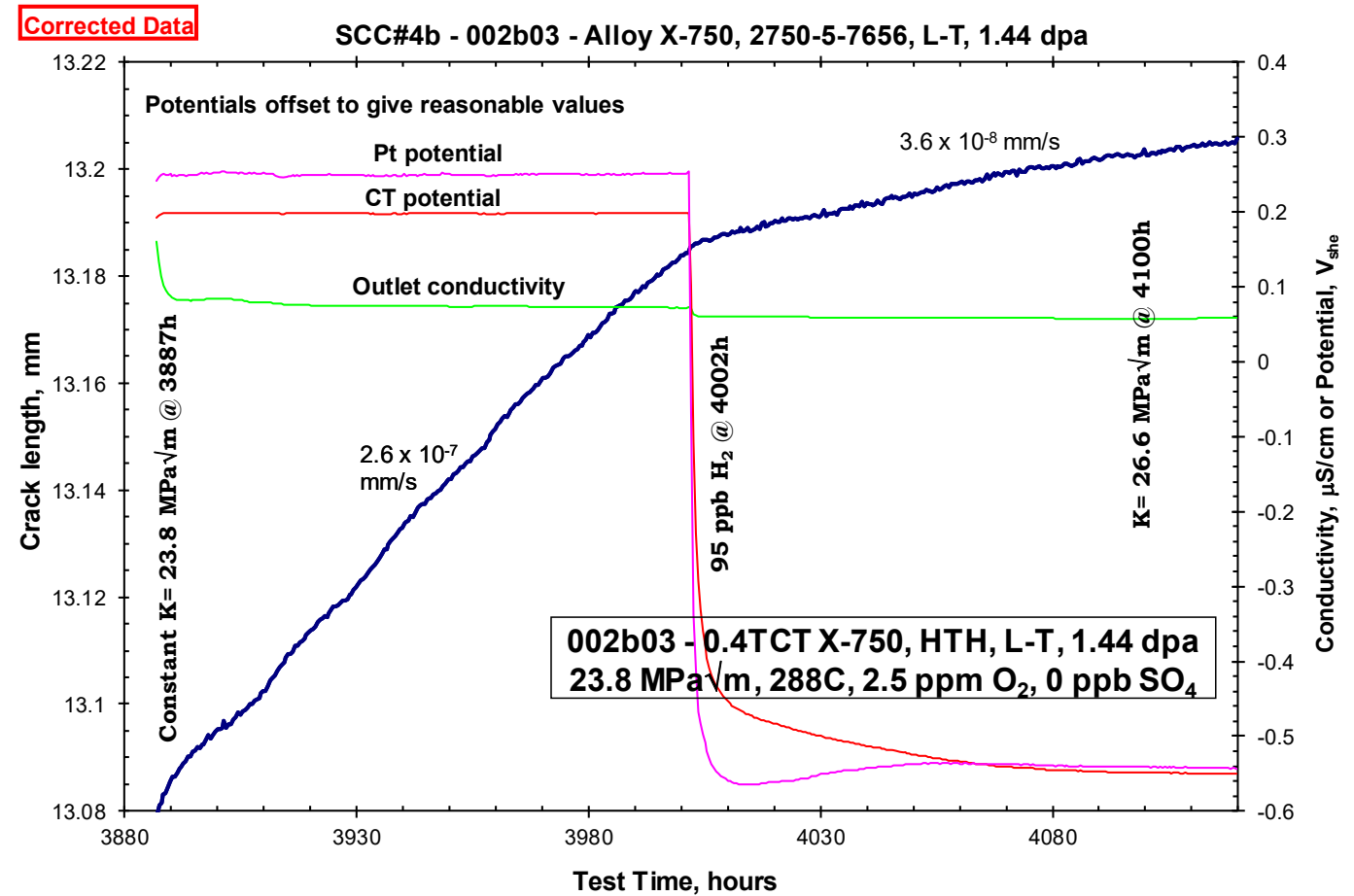

Figure 5-32: IASCC CGR following return to NWC test conditions for specimen 10A0002B03 of alloy $\mathrm{X}-750$ irradiated to 1.44 dpa.

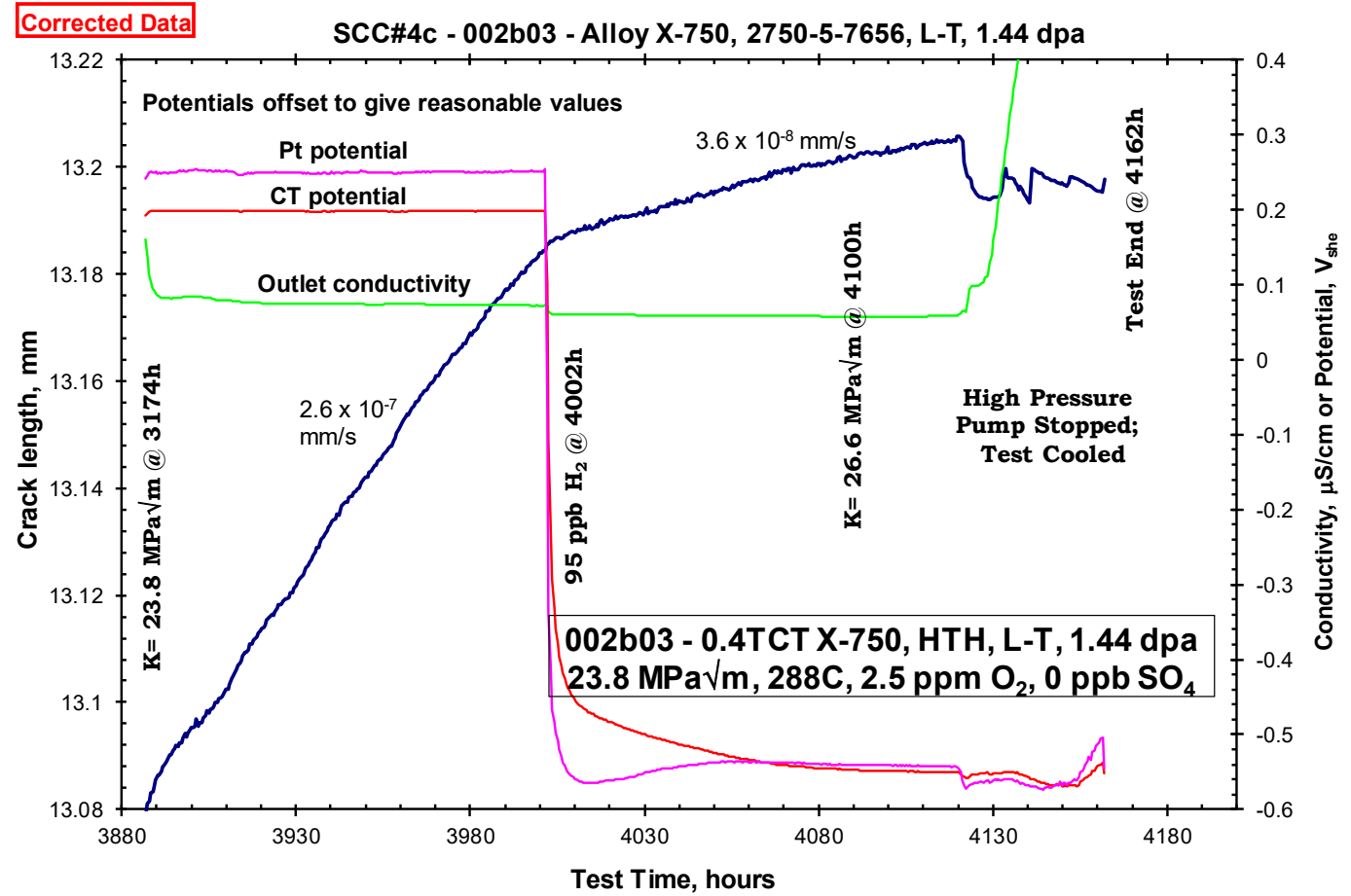

Figure 5-33: Final steps of IASCC CGR test for specimen 10A0002B03 of alloy X-750 irradiated to 1.44 dpa. 


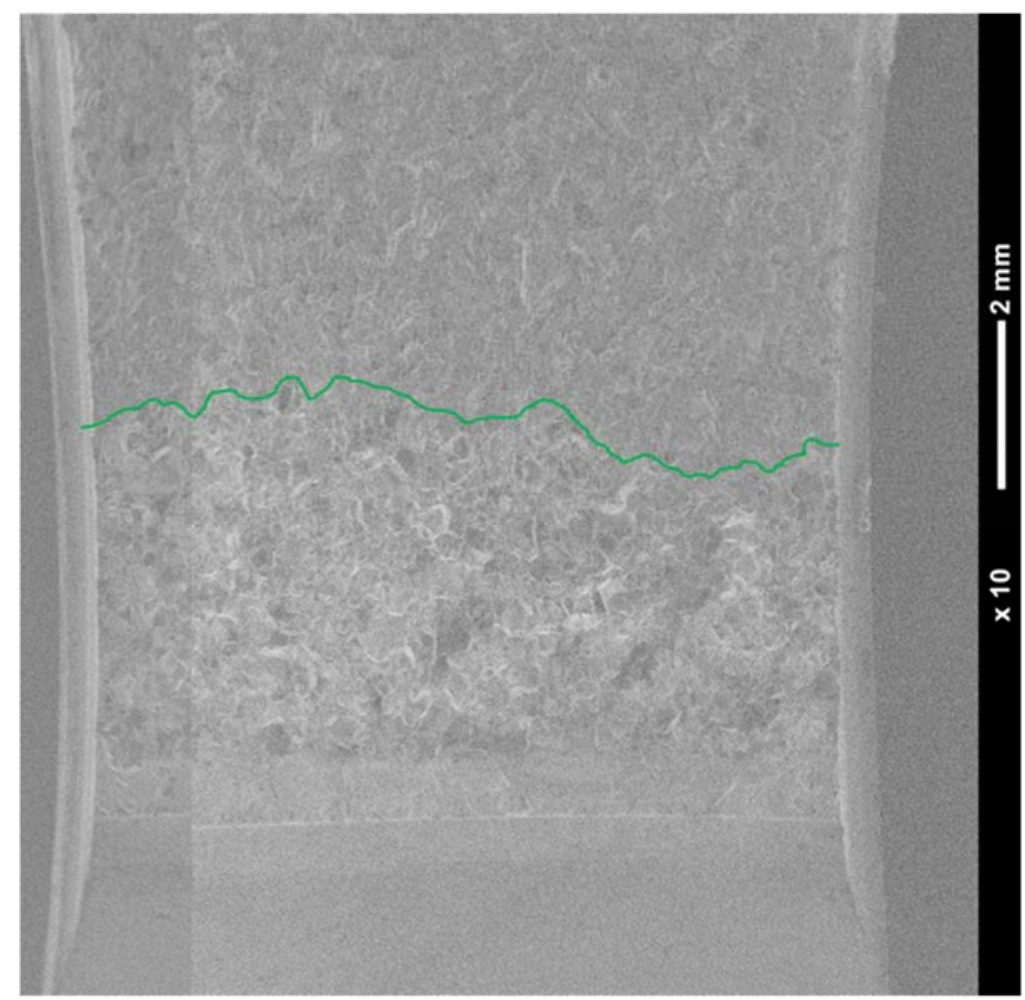

Figure 5-34: SEM micrograph of fracture surface for specimen 10A0002B03 of alloy $\mathrm{X}-750$ irradiated to 1.44 dpa.

\subsubsection{Specimen 10A0002B09 (1.54 dpa) alloy X-750 IASCC test}

Figure 5-35 provides an overview of the response of specimen 10A0002B09, which was started in $288^{\circ} \mathrm{C}$ water with $2.5 \mathrm{ppm} \mathrm{O}_{2}$ and $10 \mathrm{ppb}$ sulfate as $\mathrm{H}_{2} \mathrm{SO}_{4}$. The in-situ fatigue pre-cracking response is shown in Figure 5-36. In each graph, the label in the outlined box represents the starting condition for that plot. Some graphs show additional K values, which represent an evolution in K resulting from the posttest correction, although some intentional changes in $\mathrm{K}$ were also made. 


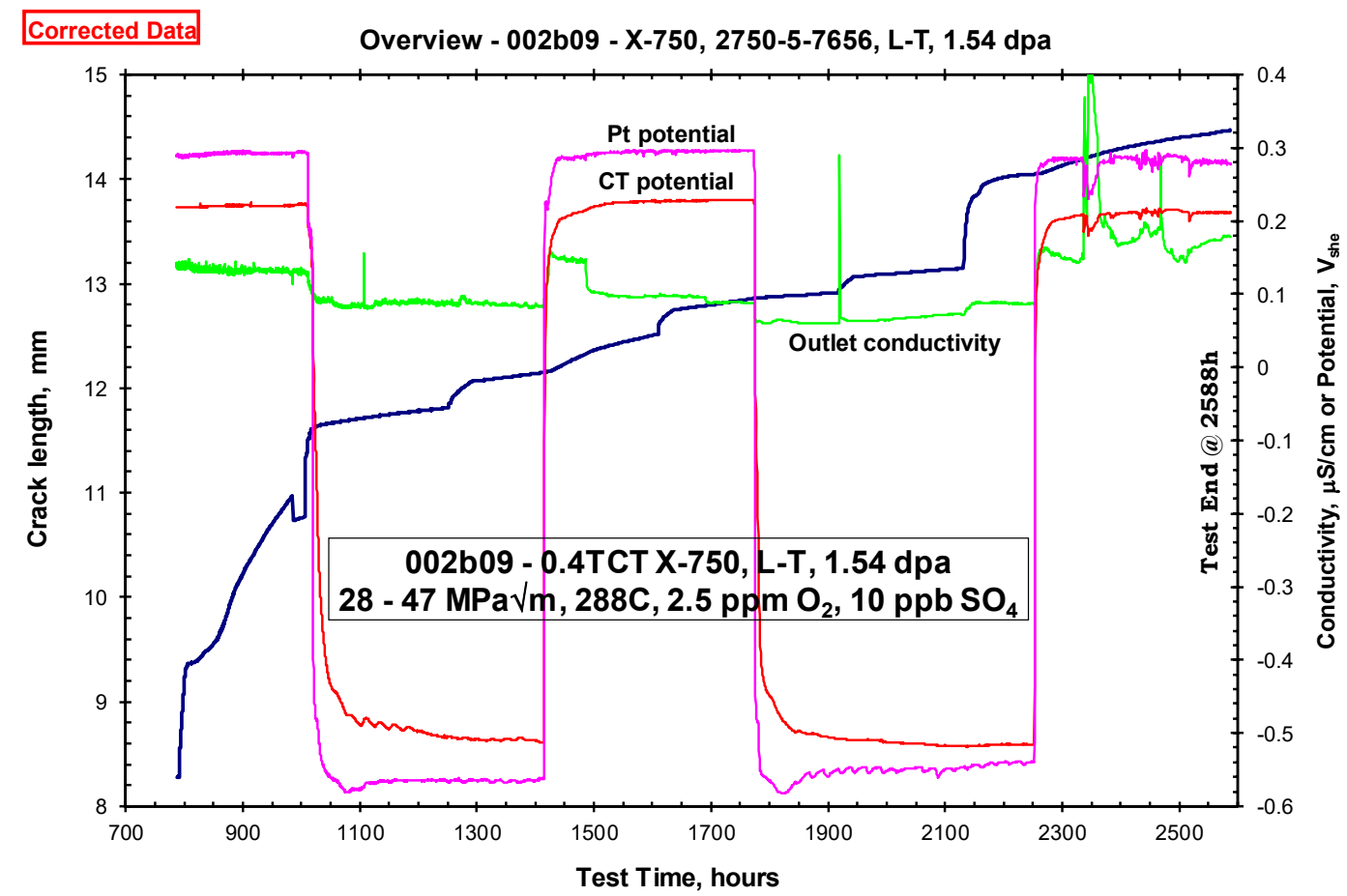

Figure 5-35: IASCC CGR test overview for specimen 10A0002B09 of alloy X-750 irradiated to 1.54 dpa.

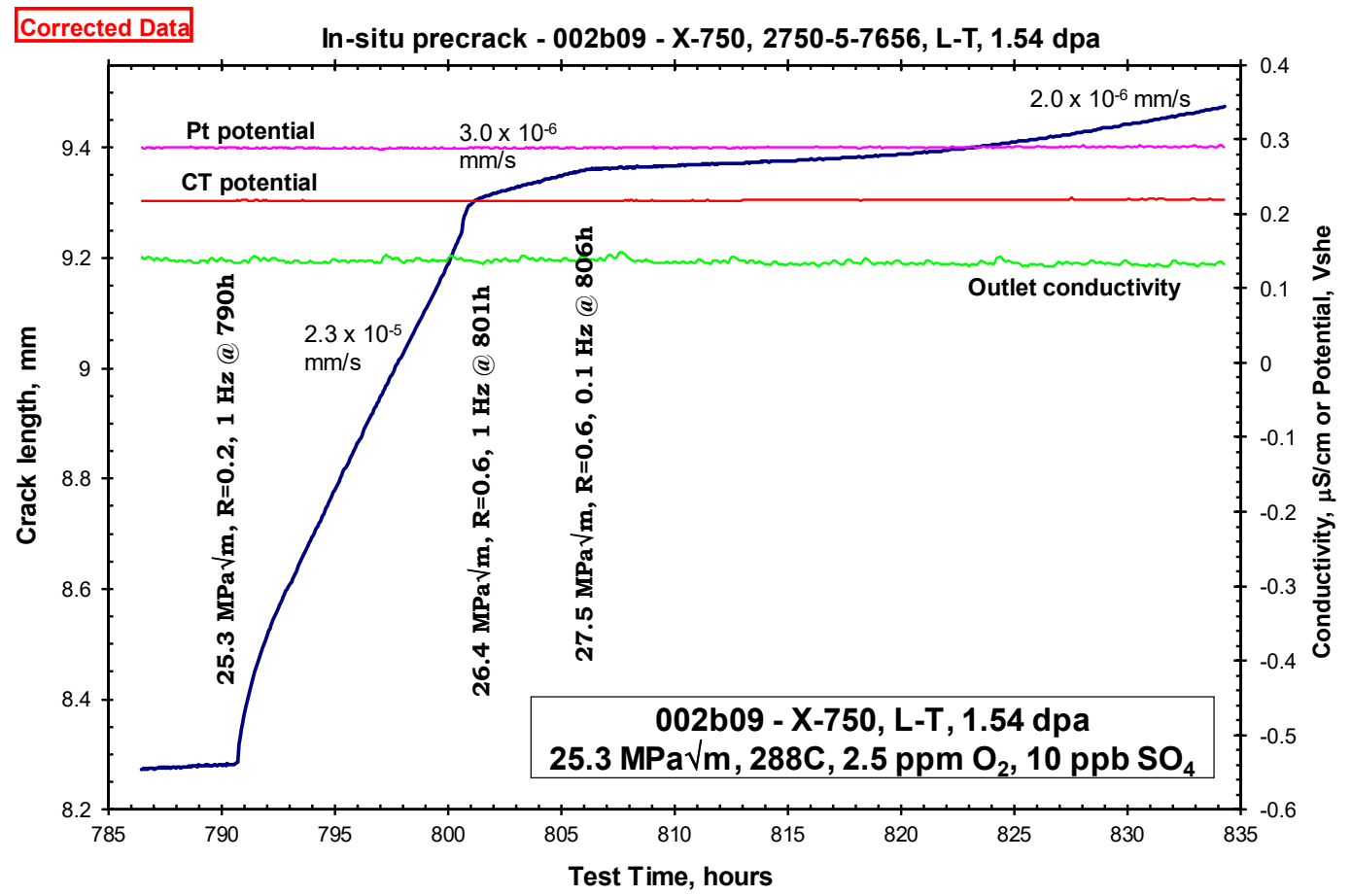

Figure 5-36: In-situ pre-fatigue steps for specimen 10A0002B09 of alloy X-750 irradiated to 1.54 dpa.

After fatigue pre-cracking, intergranular transitioning was initiated at 834 hours at $\mathrm{K}_{\max }=28 \mathrm{MPa} \sqrt{\mathrm{m}}$, loading ratio $\mathrm{R}=0.6$, frequencies of $0.01 \mathrm{~Hz}$ followed by $0.001 \mathrm{~Hz}$, and finally a 9,000 s hold at $\mathrm{K}_{\max }$ 
(Figure 5-37). Despite the decrease in loading frequency, the CGR increased at each step, indicative of a shift to fully intergranular cracking. After shifting to constant K (no cycling) at 889 hours (Figure 5-38), the growth rate decreased somewhat, and the subsequent response was well-behaved with a growth rate of $2.2 \times 10^{-6} \mathrm{~mm} / \mathrm{s}$. There was a loss of hydraulic power and load in the vicinity of 1,000 hours. After reloading and briefly cycling, a simultaneous change to constant $\mathrm{K}$ (no cycling) and $95 \mathrm{ppb}_{2}$ was made, producing a growth rate of $2 \times 10^{-7} \mathrm{~mm} / \mathrm{s}$ (about $11 \mathrm{X}$ lower).

At 1,252 hours, gentle cycling was introduced (Figure 5-39), and the CGR increased to $1.1 \times 10^{-6}$ $\mathrm{mm} / \mathrm{s}$. On returning to constant $\mathrm{K}$ at 1,292 hours, the growth rate dropped back to $2 \times 10^{-7} \mathrm{~mm} / \mathrm{s}$. At 1,416 hours, a change to $2.5 \mathrm{ppm} \mathrm{O}_{2}$ was made at 2492 hours, resulting in an increase in growth rate to $7.6 \times 10^{-}$ ${ }^{7} \mathrm{~mm} / \mathrm{s}$. The injection of sulfate was stopped at 485 hours, and the growth rate dropped by $2 \mathrm{X}$ to $3.6 \times 10^{-}$ ${ }^{7} \mathrm{~mm} / \mathrm{s}$.

Gentle cycling was again briefly introduced at 1,607 hours (Figure 5-40), then the loading was returned to constant $\mathrm{K}$ (no cycling) conditions; the growth rate was somewhat reduced from the value

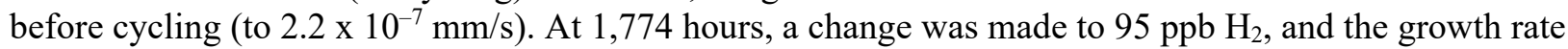
dropped somewhat to $9.4 \times 10^{-8} \mathrm{~mm} / \mathrm{s}$. Gentle cycling was again briefly introduced at 1,917 hours, then the loading was returned to constant $\mathrm{K}$ (no cycling) conditions, and the growth rate was similar to the value before cycling.

At 2,132 hours (Figure 5-41), injection of $10 \mathrm{ppb}$ sulfate was started, and gentle cycling was initiated at loading ratio $\mathrm{R}=0.5$ and a frequency of $0.001 \mathrm{~Hz}$. About $1 \mathrm{~mm}$ of growth occurred during this initial phase, as well as subsequent phases at $\mathrm{R}=0.6$. At 2,214 hours, a 9,000s hold at $\mathrm{K}_{\max }$ was introduced, and the growth rate was $1.1 \times 10^{-7} \mathrm{~mm} / \mathrm{s}$.

A change to $2.5 \mathrm{ppm} \mathrm{O}_{2}$ was made at 2,252 hours (Figure 5-42), and the CGR increased by $5 \mathrm{X}$ to 5.7 $\mathrm{x} 10^{-7} \mathrm{~mm} / \mathrm{s}$. After changing to constant $\mathrm{K}$ (no cycling) at 2,323 hours, the growth rate dropped somewhat to $4.6 \times 10^{-7} \mathrm{~mm} / \mathrm{s}$, then decayed a bit over time to $2.3 \times 10^{-7} \mathrm{~mm} / \mathrm{s}$.

The test ended at 2,588 hours, and a macro photograph of the fracture surface is shown in Figure $5-43$. The average intergranular crack depth on the fracture surface is about $62 \%$ higher than was indicated by DCPD. The crack length, CGR, and K data were corrected in all plots. The post-test corrected $\mathrm{K}$ values were higher than intended during testing because the error was moderately large andequally important - the amount of total crack extension was moderately large. Table 5-4 is a summary of all the CGRs and related conditions under periods of constant applied $\mathrm{K}$ for this test.

Table 5-4: Constant K IASCC CGR and related conditions for specimen 10A0002B09 of alloy X750 irradiated to $1.54 \mathrm{dpa}$; blue highlights indicate higher confidence data.

\begin{tabular}{|c|c|c|c|c|c|c|c|}
\hline $\begin{array}{c}\text { Test } \\
\text { Hours }\end{array}$ & $\begin{array}{c}\mathbf{K} \\
(\mathbf{M P a V m})\end{array}$ & Chemistry & Sulfate & $\begin{array}{c}\text { Outlet } \\
\text { Cond } \\
(\boldsymbol{\mu} \mathbf{S} / \mathbf{c m})\end{array}$ & $\begin{array}{c}\text { Time } \\
\text { Increment } \\
(\mathbf{h r})\end{array}$ & $\begin{array}{c}\text { Growth } \\
\text { Increment } \\
(\mathbf{m m})\end{array}$ & $\begin{array}{c}\text { Average } \\
\mathbf{C G R} \\
(\mathbf{m m} / \mathbf{s})\end{array}$ \\
\hline 889 & 30 & NWC & 10 & 0.129 & 96 & 0.856 & $2.20 \mathrm{E}-06$ \\
\hline 1017 & 30 & HWC & 10 & 0.087 & 235 & 0.211 & $2.10 \mathrm{E}-07$ \\
\hline 1292 & 30 & HWC & 10 & 0.085 & 124 & 0.086 & $2.00 \mathrm{E}-07$ \\
\hline 1416 & 35 & NWC & 10 & 0.144 & 69 & 0.173 & $7.60 \mathrm{E}-07$ \\
\hline 1485 & 35 & NWC & 0 & 0.097 & 122 & 0.190 & $3.80 \mathrm{E}-07$ \\
\hline 1634 & 38 & NWC & 0 & 0.088 & 140 & 0.119 & $2.20 \mathrm{E}-07$ \\
\hline 1774 & 38 & HWC & 0 & 0.06 & 143 & 0.047 & $9.40 \mathrm{E}-08$ \\
\hline 1943 & 40 & HWC & 0 & 0.071 & 189 & 0.085 & $1.20 \mathrm{E}-07$ \\
\hline 2323 & 46 & NWC & 0 & 0.175 & 265 & 0.295 & $4.00 \mathrm{E}-07$ \\
\hline
\end{tabular}




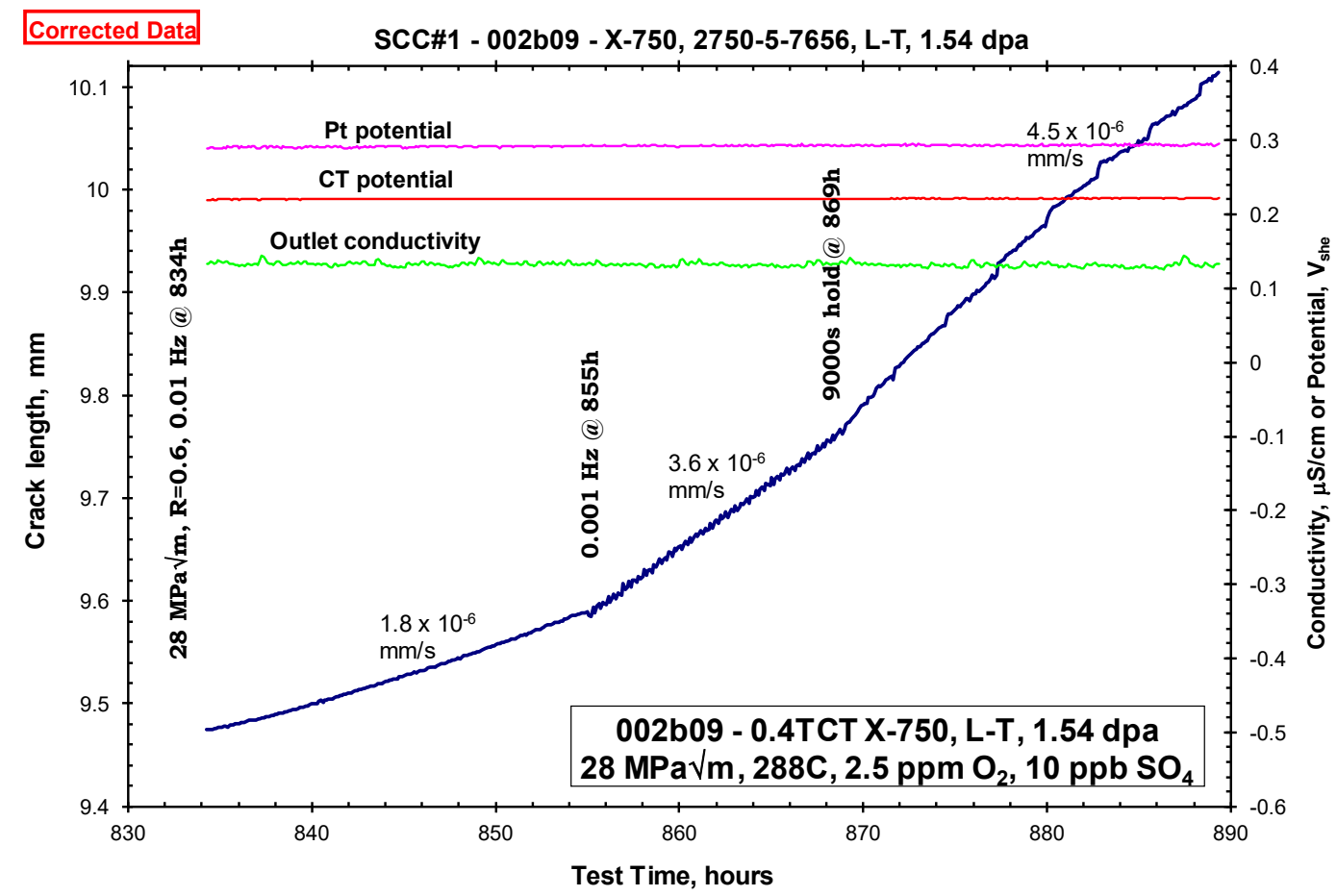

Figure 5-37: IASCC transition to intergranular fracture for specimen $10 \mathrm{A0002B09}$ of alloy $\mathrm{X}-750$ irradiated to 1.54 dpa.

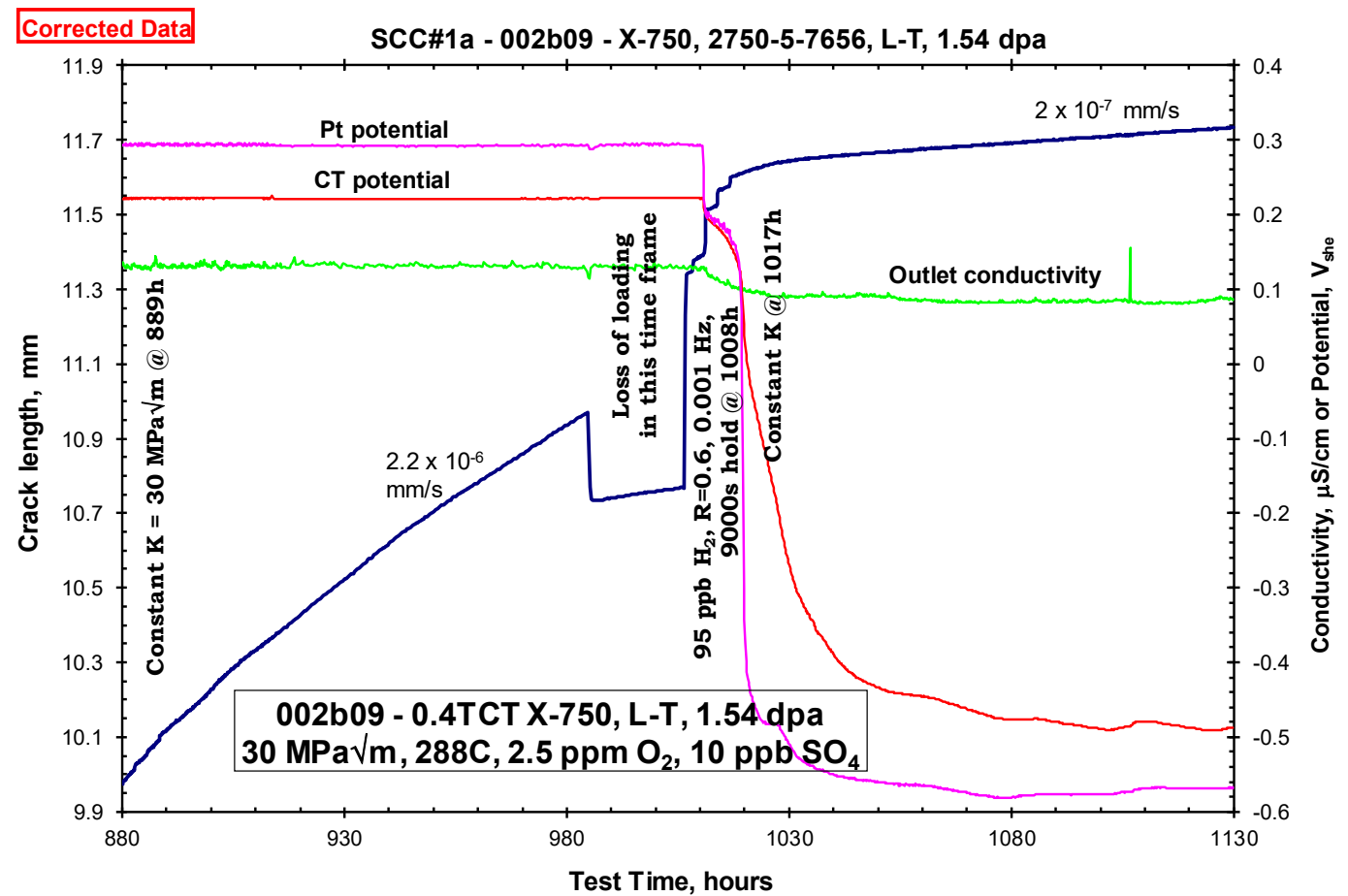

Figure 5-38: Initial IASCC CGR response at constant $K$ for NWC and HWC in specimen 10A0002B09 of alloy X-750 irradiated to $1.54 \mathrm{dpa}$. 


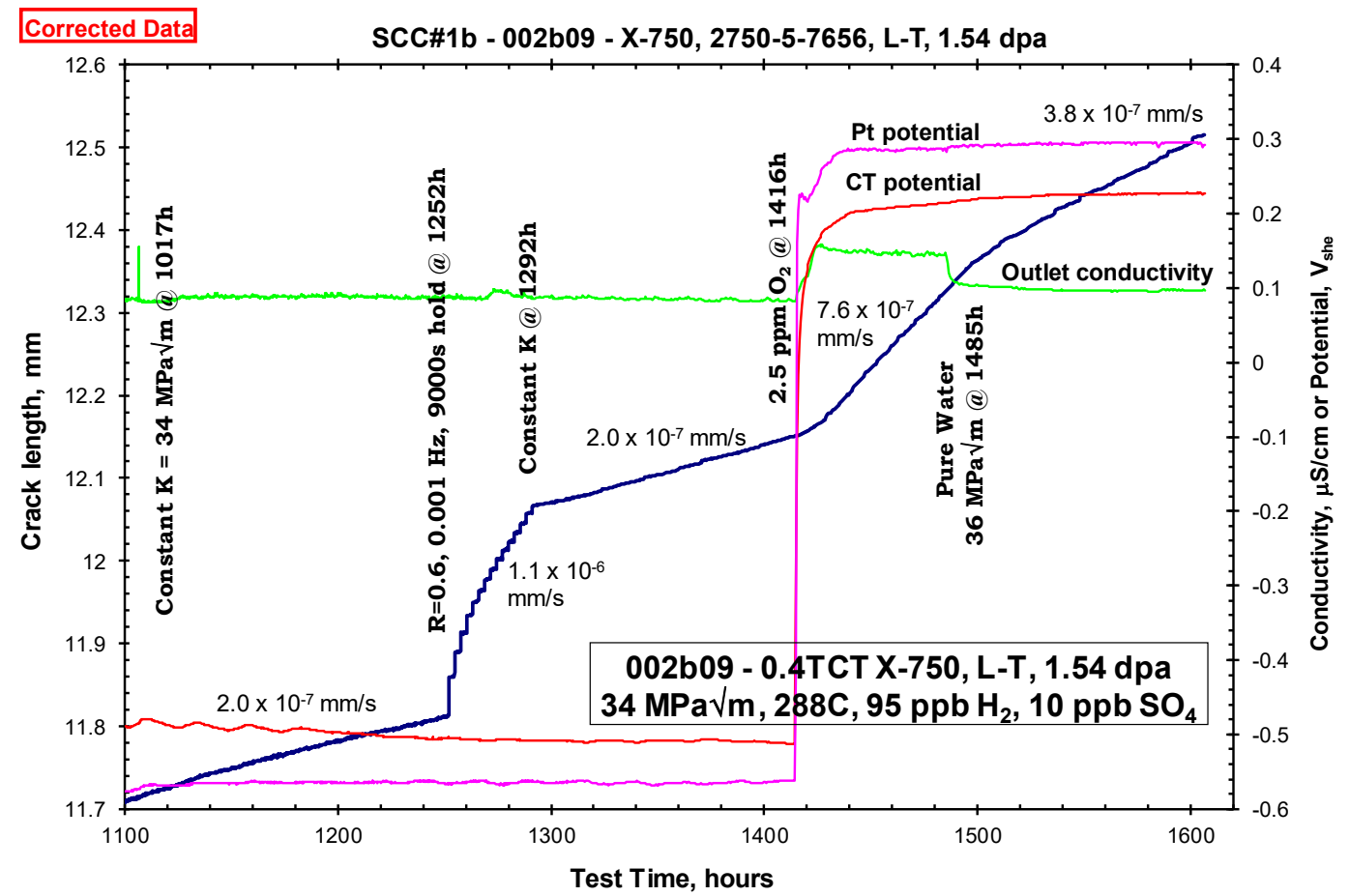

Figure 5-39: IASCC CGR test at constant $K$ under HWC and NWC for specimen 10A0002B09 of alloy $\mathrm{X}-750$ irradiated to 1.54 dpa.

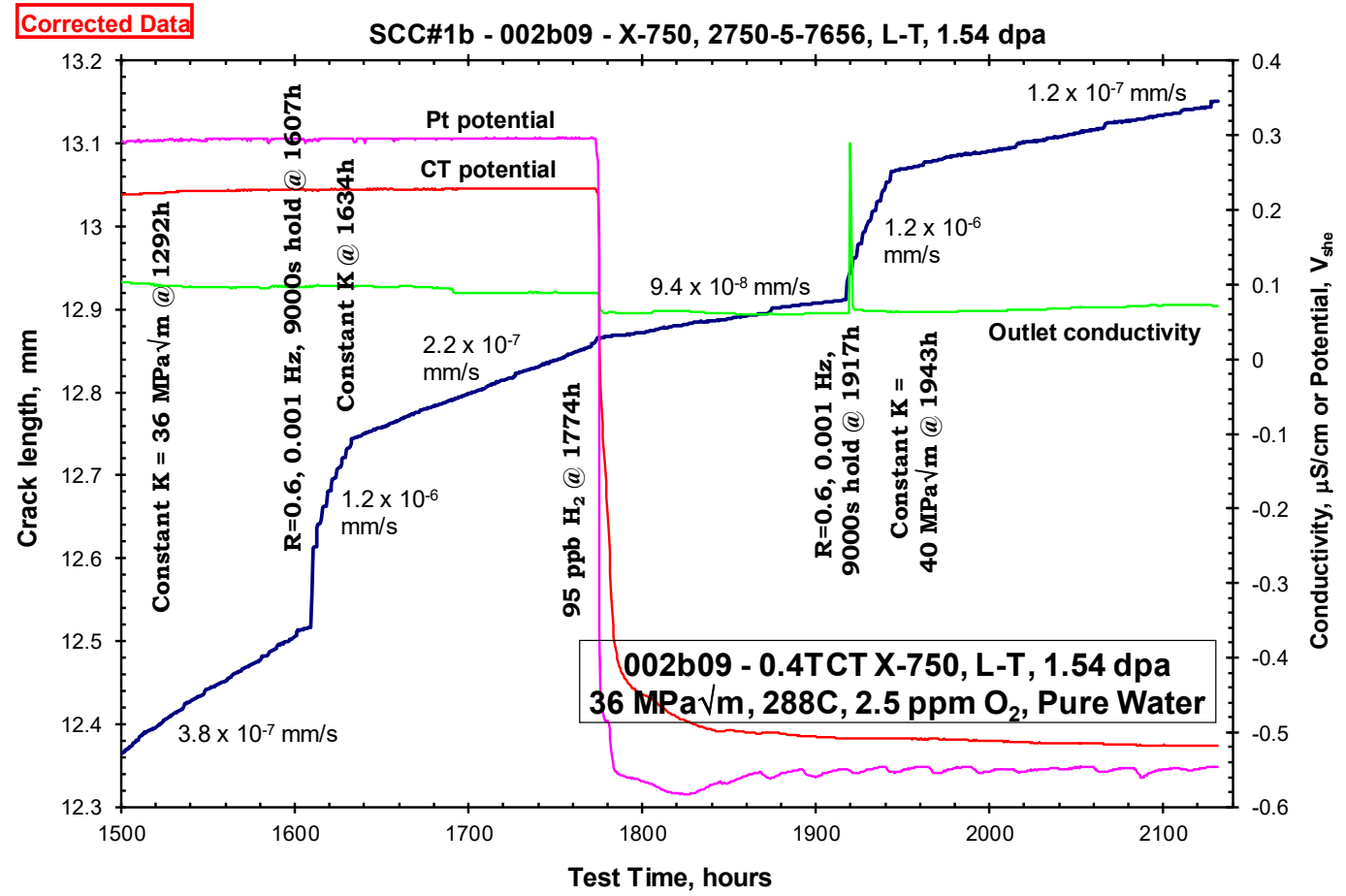

Figure 5-40: IASCC CGR response during and after periods of gentle cycling to straighten the crack front for specimen 10A0002B09 of alloy X-750 irradiated to $1.54 \mathrm{dpa}$. 


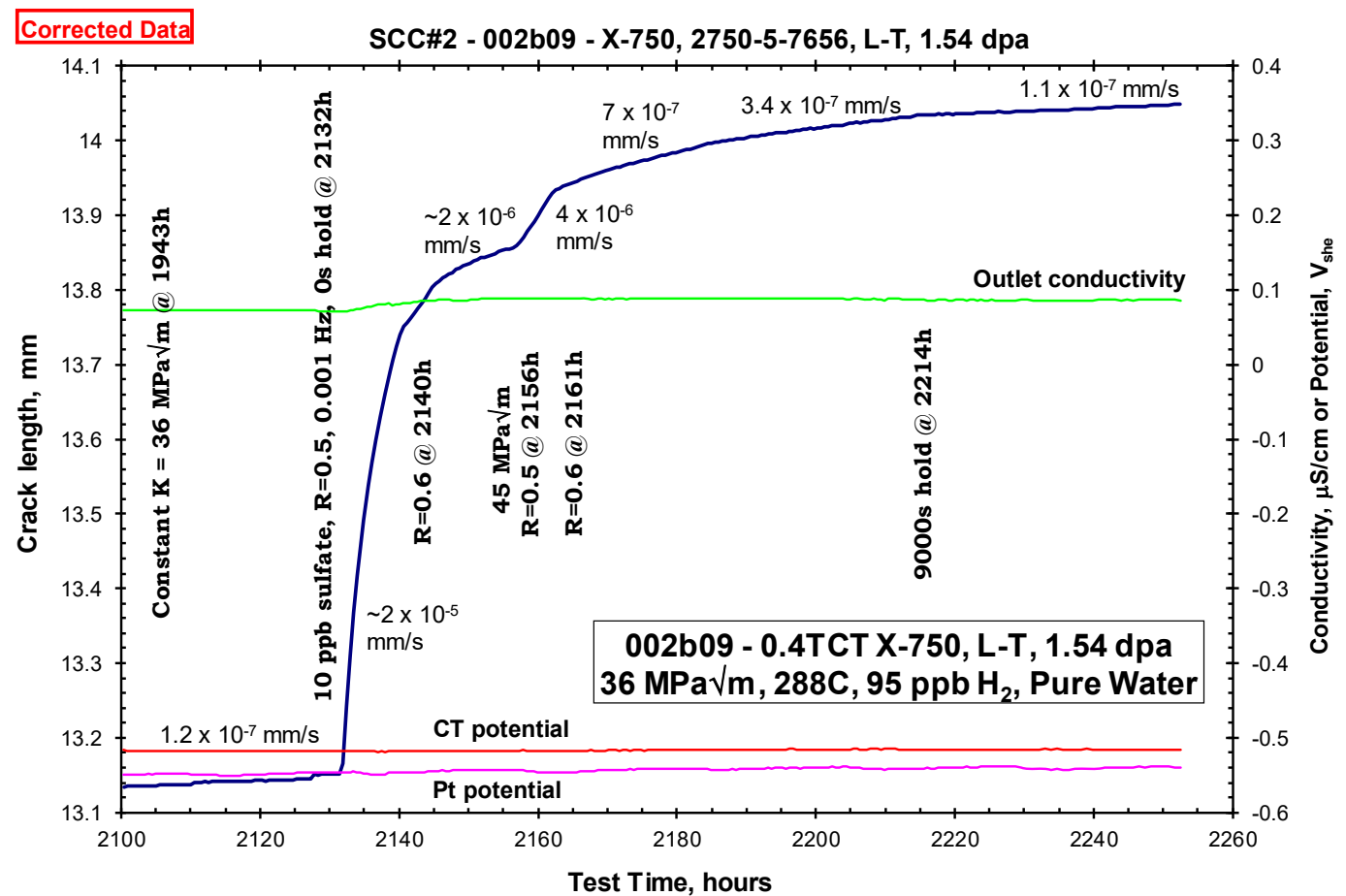

Figure 5-41: IASCC CGR response under $H W C$ at higher $K$ and gentle cycling for specimen $10 \mathrm{~A} 0002 \mathrm{~B} 03$ of alloy $\mathrm{X}-750$ irradiated to $1.54 \mathrm{dpa}$.

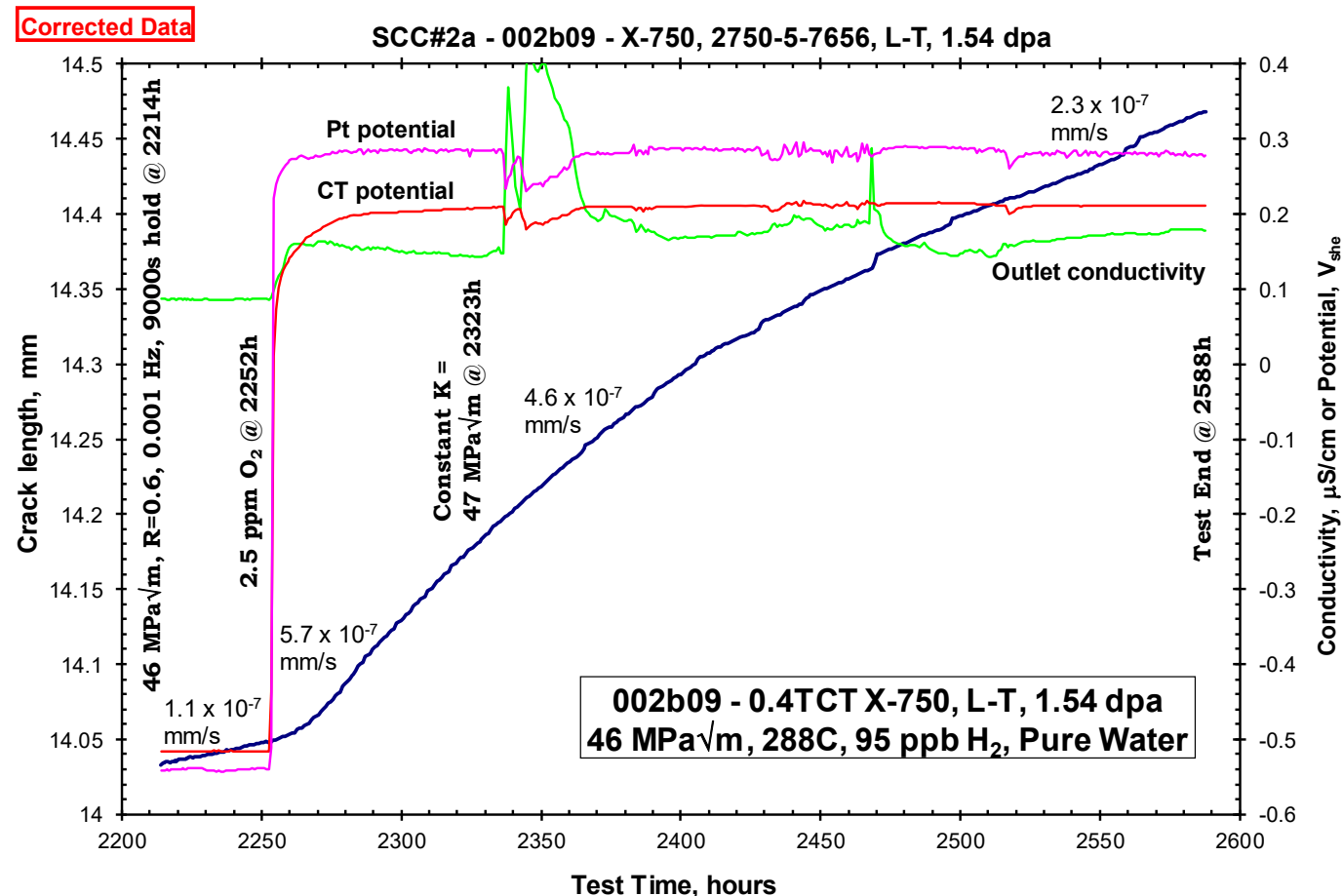

Figure 5-42: Final steps of IASCC CGR test for specimen 10A0002B03 of alloy X-750 irradiated to 1.54 dpa. 


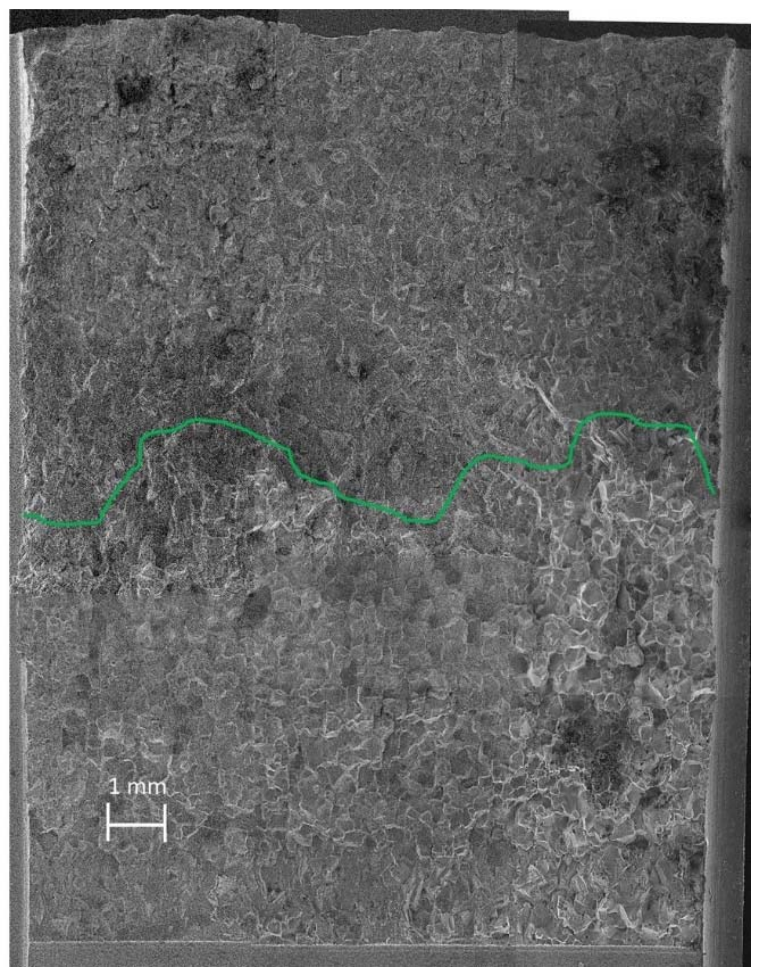

Figure 5-43: SEM micrograph of fracture surface for specimen 10A0002B09 of alloy X-750 irradiated to 1.54 dpa.

\subsection{Alloy XM-19 IASCC Testing Details}

\subsubsection{Medium Fluence XM-19 IASCC CGR Tests}

Specimens 10A0001B02 and 10A0001B03 were both irradiated to fast-neutron damage levels of approximately $0.29 \mathrm{dpa}$. Irradiation temperatures are estimated to be in the range of $349-360{ }^{\circ} \mathrm{C}$ for both specimens, as shown in Table 3-5. It is noted that specimen 10A0001B02 was utilized for both IASCC testing and fracture toughness testing, so the IASCC CGR test is relatively short; this is because during the IASCC test, a pull rod seal failed, and it was decided to utilize the remainder of the ligament for an fracture toughness test, since a consistent CGR had been demonstrated under both NWC and HWC at constant K. Details of these two IASCC tests follow; in all plots, the blue line tracks crack length vs time (CGR).

\subsubsection{Specimen 10A0001B02 (0.29 dpa) XM-19 IASCC test 1}

Figure 5-44 provides an overview of the IASCC CGR response of specimen 10A001B02, which was started in $288^{\circ} \mathrm{C}$ pure water with $2.5 \mathrm{ppm} \mathrm{O}_{2}$. The in-situ fatigue pre-cracking response is shown in Figure 5-45. In each graph, the label in the outlined box represents the starting condition for that plot.

After fatigue pre-cracking, intergranular transitioning was initiated at 1,740 hours at $29 \mathrm{MPa} \sqrt{\mathrm{m}}$, loading ratio $\mathrm{R}=0.6$, at frequencies of 0.01 and $0.001 \mathrm{~Hz}$, with 9,000s hold at $\mathrm{K}_{\max }$ (Figure 5-46). At 193 hours, injection of $10 \mathrm{ppb}$ sulfate as $\mathrm{H}_{2} \mathrm{SO}_{4}$ was started, with no change in growth rate. At 2,025 hours, a change to constant $\mathrm{K}$ (no cycling) was made, again with no change in growth rate.

A change to $95 \mathrm{ppb}_{2}$ was made at 2,170 hours (Figure 5-47), and the growth rate dropped to $\sim 0$ $\mathrm{mm} / \mathrm{s}$, which was sustained over $\sim 500$ hours. At this point (2,673 hours), the pull rod seal failed, and the 
water in the autoclave leaked out. Figure 5-48 is a macro photograph of the fracture surface, which shows where the SCC crack front ended. The average intergranular crack depth on the fracture surface is about $28 \%$ higher than was indicated by DCPD. The crack length, crack growth rate, and K data were corrected in all plots. The post-test corrected $\mathrm{K}$ values were only a bit higher than intended during testing because the error was only moderate and the total SCC crack extension was limited. Table 5-5 is a summary of all the CGRs and related conditions under periods of constant applied $\mathrm{K}$ for this test.

Table 5-5: Constant K IASCC CGR and related conditions for specimen 10A0001B02 of XM-19 irradiated to 0.29 dpa.

\begin{tabular}{|c|c|c|c|c|c|c|c|}
\hline $\begin{array}{c}\text { Test } \\
\text { Hours }\end{array}$ & $\begin{array}{c}\mathbf{K} \\
(\mathbf{M P a V m})\end{array}$ & Chemistry & Sulfate & $\begin{array}{c}\text { Outlet } \\
\text { Cond } \\
(\mu \mathbf{S} / \mathbf{c m})\end{array}$ & $\begin{array}{c}\text { Time } \\
\text { Increment } \\
(\mathbf{h r})\end{array}$ & $\begin{array}{c}\text { Growth } \\
\text { Increment } \\
(\mathbf{m m})\end{array}$ & $\begin{array}{c}\text { Average } \\
\mathbf{C G R} \\
(\mathbf{m m} / \mathbf{s})\end{array}$ \\
\hline 2025 & 29 & NWC & 0 & 0.16 & 145 & 0.168 & $3.30 \mathrm{E}-07$ \\
\hline 2170 & 30 & HWC & 0 & 0.082 & 503 & 0.007 & $1.00 \mathrm{E}-09$ \\
\hline
\end{tabular}

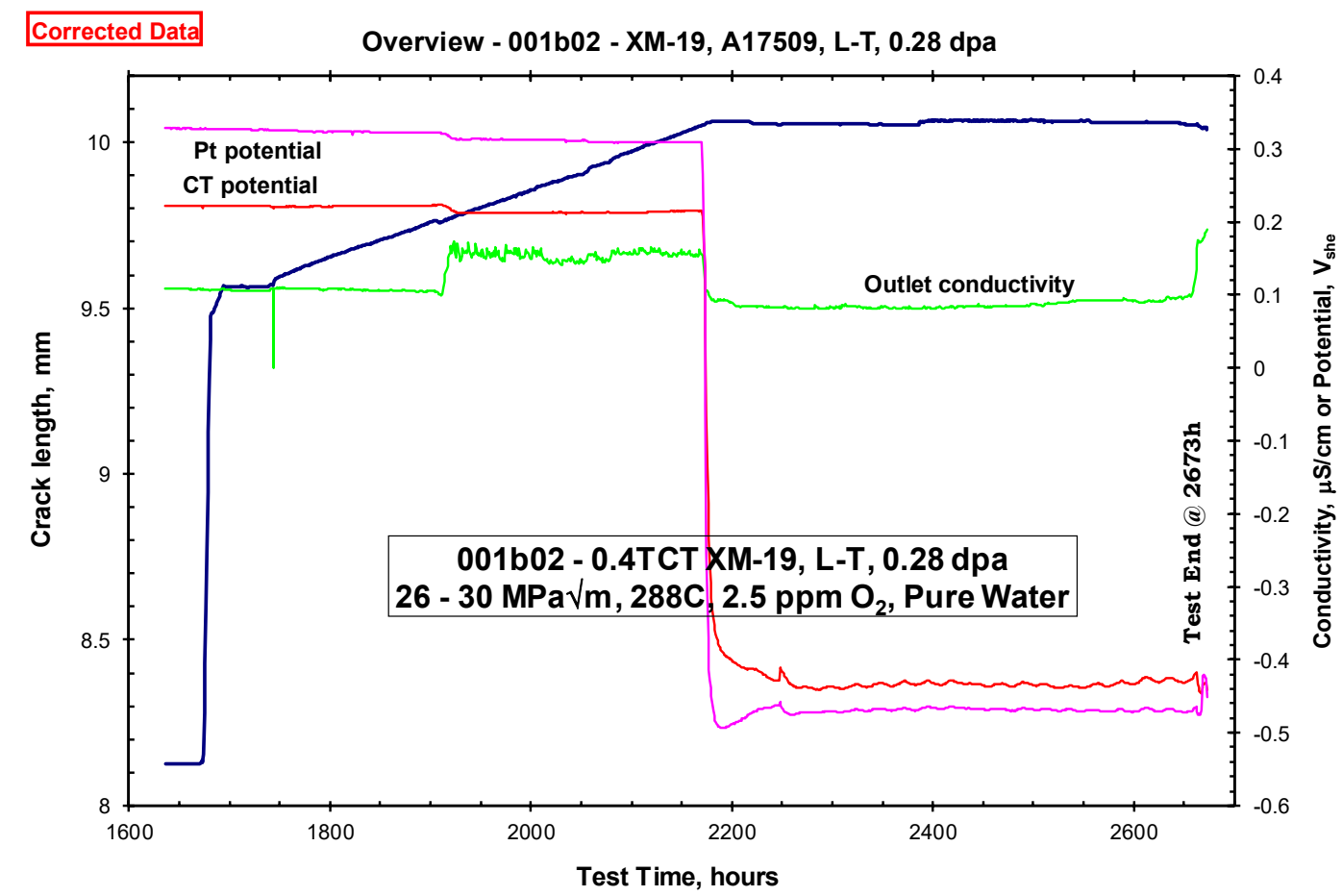

Figure 5-44: IASCC CGR test overview for specimen 10A0001B02 of XM-19 irradiated to 0.29 dpa. 


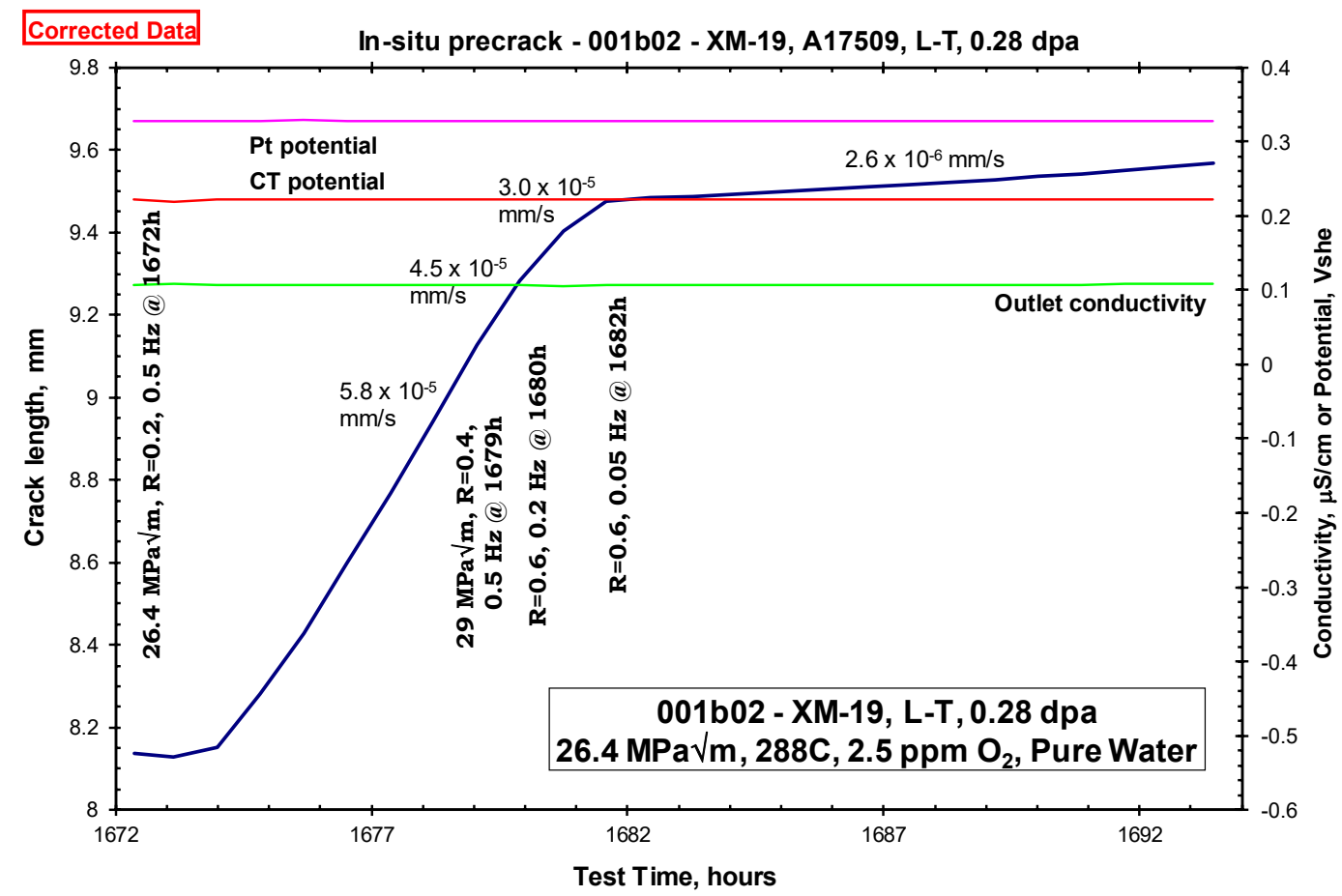

Figure 5-45: In-situ fatigue pre-cracking for IASCC CGR test of specimen 10A0001B02 of XM-19 irradiated to 0.29 dpa.

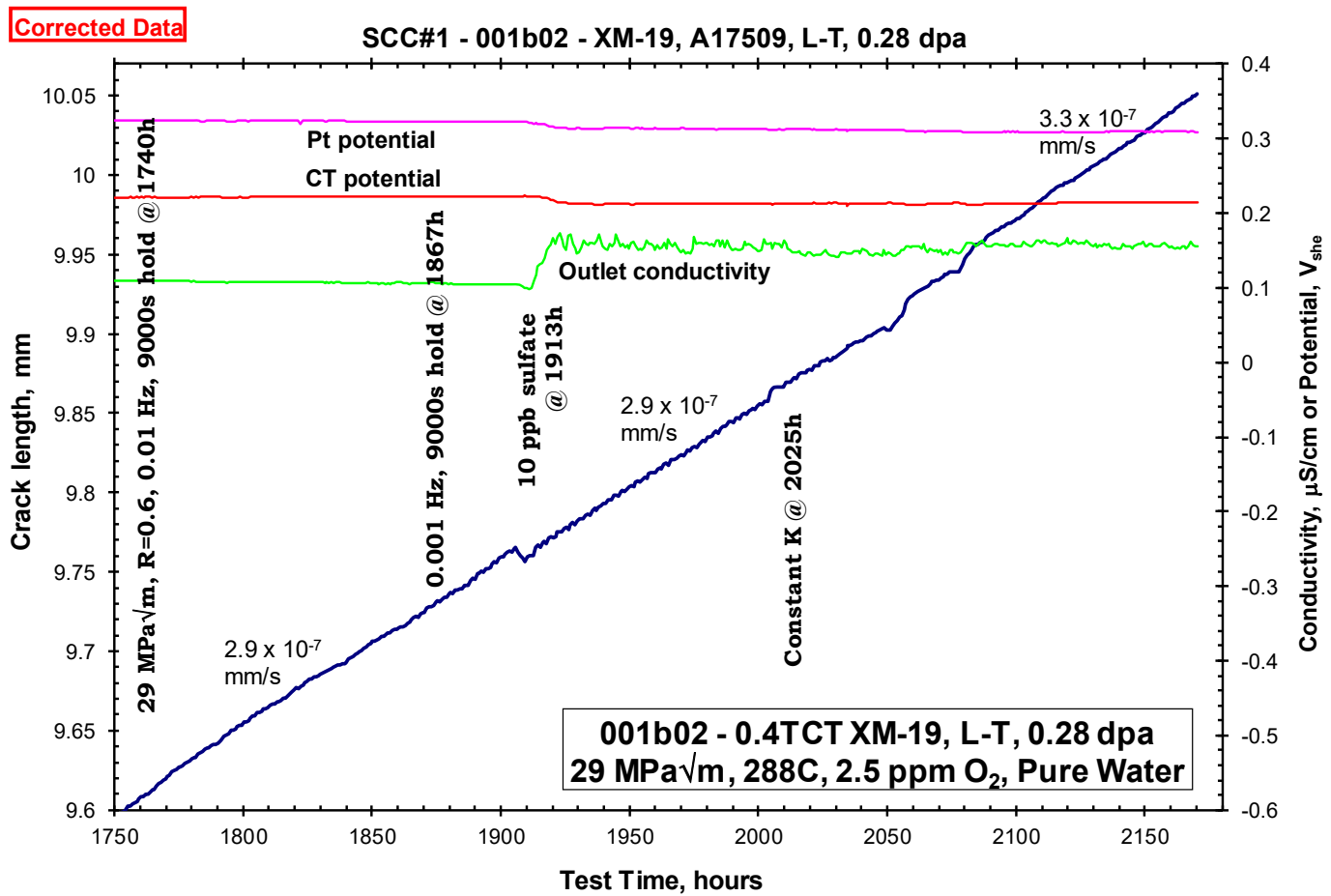

Figure 5-46: IASCC CGR response under NWC and constant $K$ for specimen 10A0001B02 of XM19 irradiated to 0.29 dpa. 


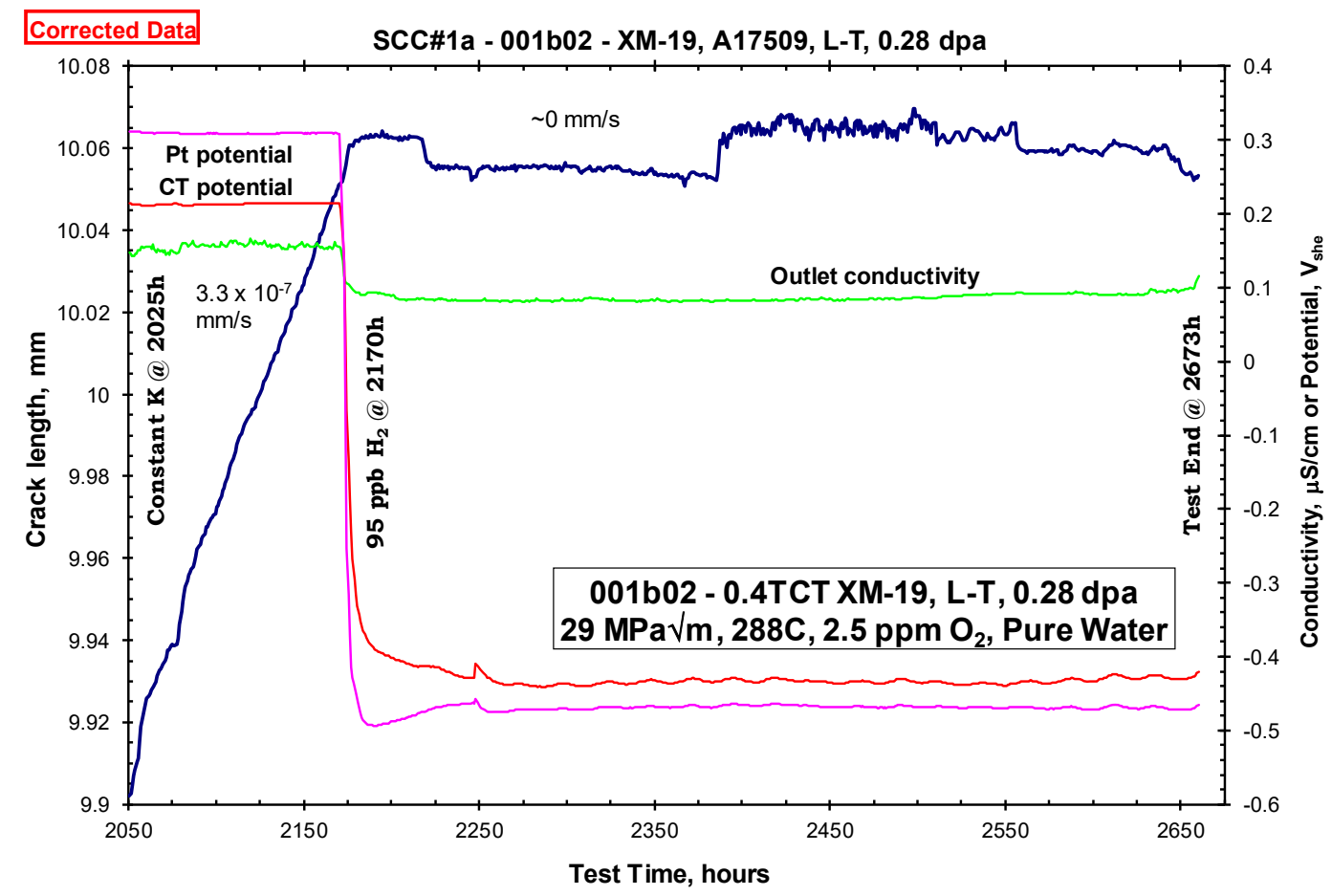

Figure 5-47: IASCC CGR response under HWC and constant $K$ for specimen 10A0001B02 of XM19 irradiated to 0.29 dpa.

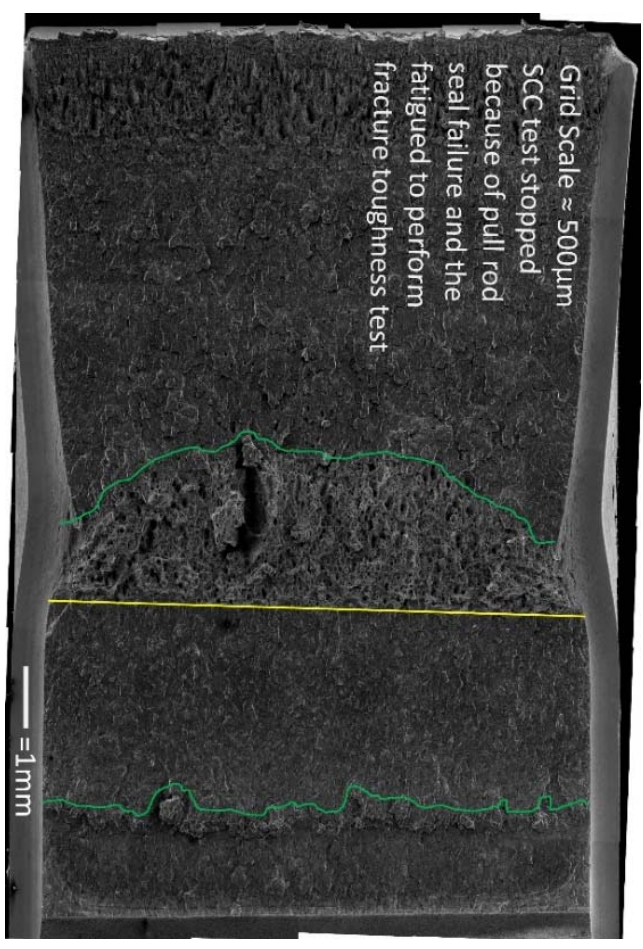

Figure 5-48: SEM micrograph of fracture surface for specimen $10 \mathrm{A0001B02}$ of XM-19 irradiated to 0.29 dpa; IASCC CGR test end is marked by lower green line. 


\subsubsection{Specimen 10A0001B03 (0.29 dpa) XM-19 IASCC test 2}

Figure 5-49 provides an overview of the response of specimen 10A0001B03, which was started in $288^{\circ} \mathrm{C}$ pure water with $2.5 \mathrm{ppm} \mathrm{O}_{2}$. The in-situ fatigue pre-cracking response is shown in Figure 5-50. In each graph, the label in the outlined box represents the starting condition for that plot. Some graphs show additional $\mathrm{K}$ values, which represent an evolution in $\mathrm{K}$ resulting from the post-test correction, although some intentional changes in $\mathrm{K}$ were also made.

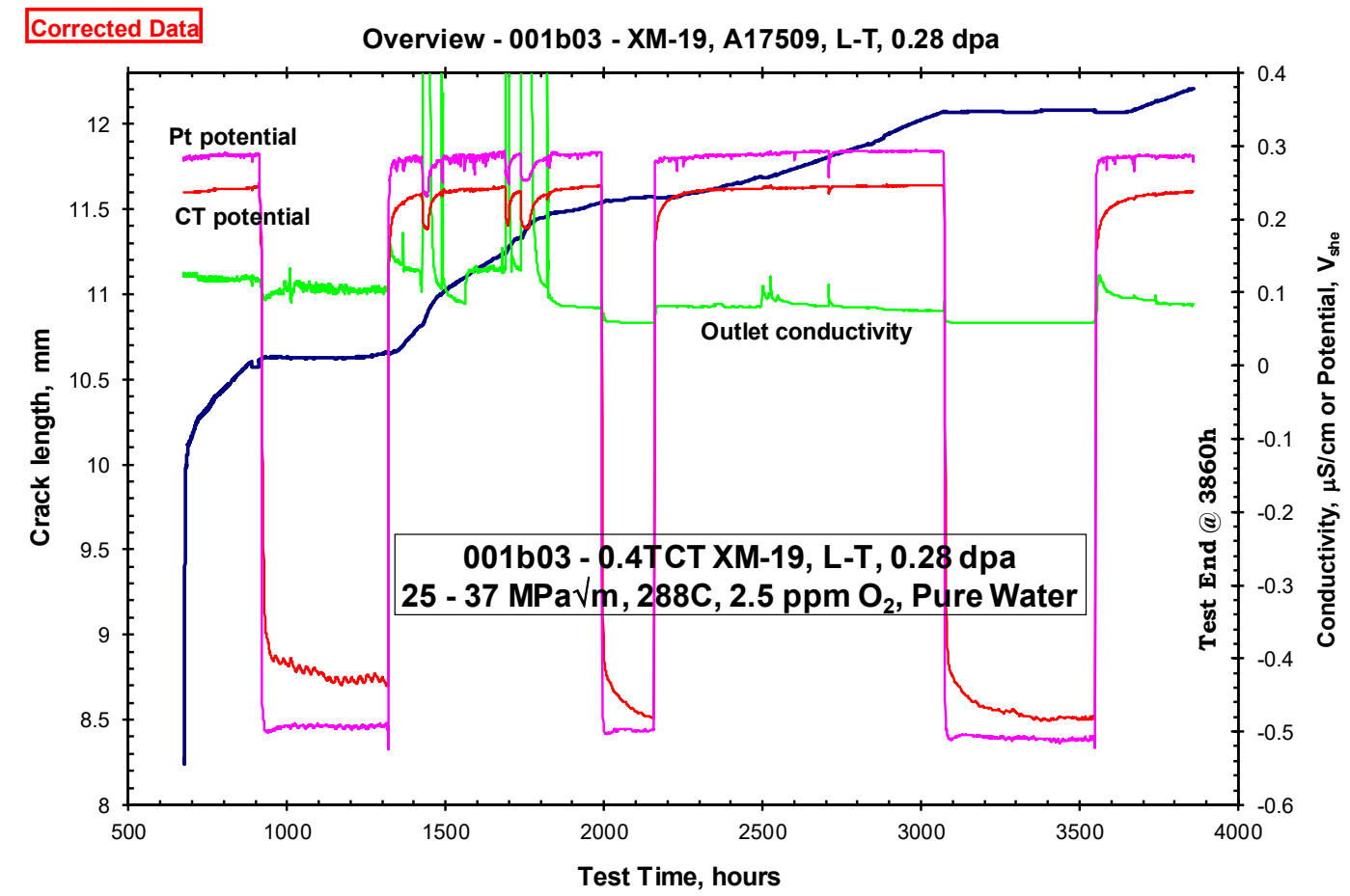

Figure 5-49: IASCC CGR test overview for specimen 10A0001B03 of XM-19 irradiated to 0.29 dpa. 


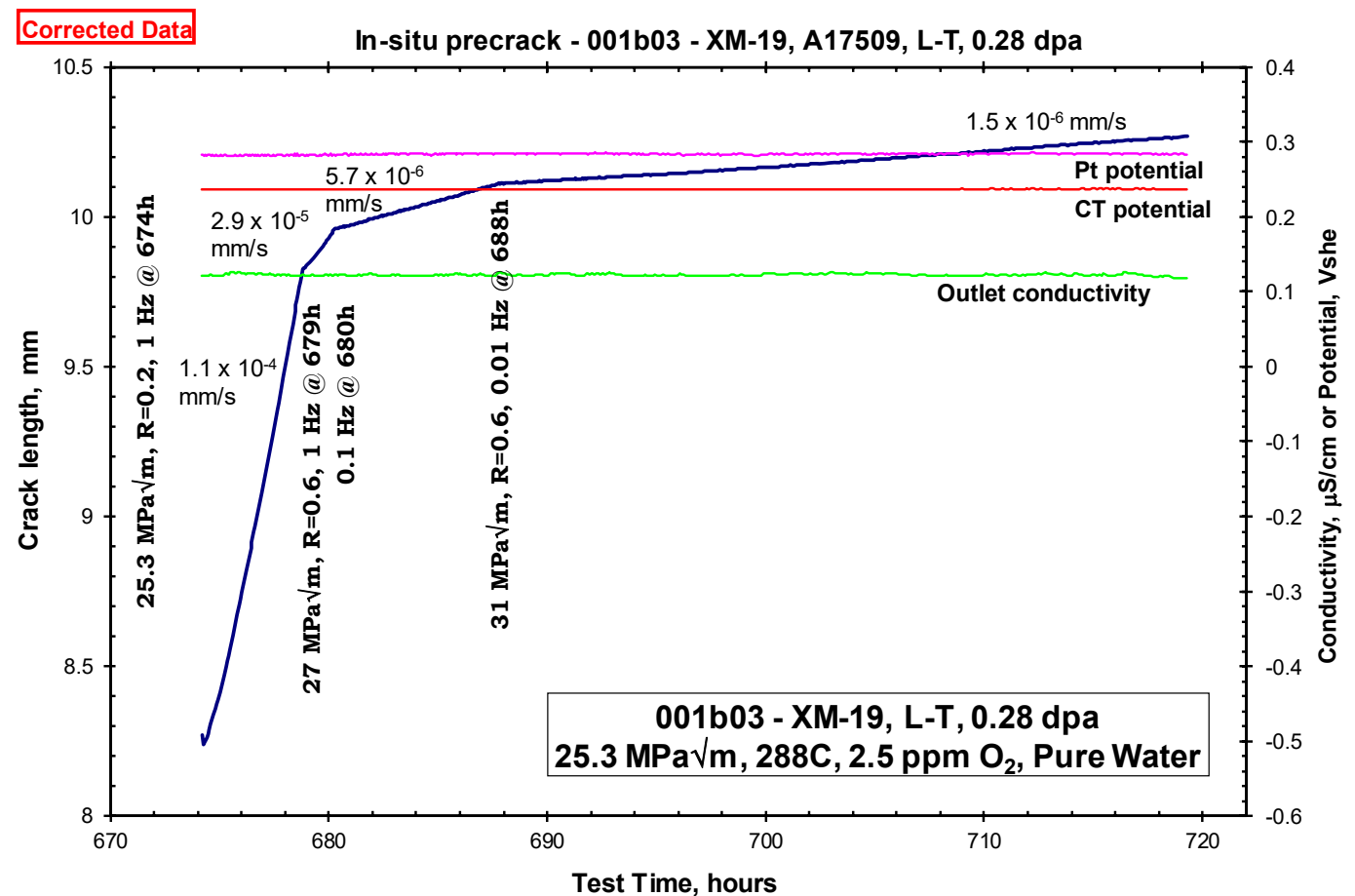

Figure 5-50: In-situ pre-fatigue for IASCC test of specimen 10A0001B03 of XM-19 irradiated to 0.29 dpa.

After fatigue pre-cracking, intergranular transitioning was initiated at 834 hours with $\mathrm{K}_{\max }=28$ $\mathrm{MPa} \sqrt{\mathrm{m}}$, loading ratio $\mathrm{R}=0.6$, and frequencies of $0.01 \mathrm{~Hz}$ followed by $0.001 \mathrm{~Hz}$, then with a $9,000 \mathrm{~s}$ hold at $\mathrm{K}_{\max }$ (Figure 5-51). At about 900 hours, the load was reduced, and was restored at 911 hours (Figure 5-52). At 913 hours, a change was made to $75 \mathrm{ppb} \mathrm{H}_{2}$; then at 925 hours, a change to constant $\mathrm{K}$ (no cycling) was made, and no growth occurred. The cyclic variation in crack length under HWC is likely a result of fluctuations in the ambient laboratory temperature. The data associated with both changes would have been clearer if only one change was made and the response observed.

Gentle cycling was initiated at 1,155 hours, and it produced a growth rate of $1.2 \times 10^{-7} \mathrm{~mm} / \mathrm{s}$ (Figure 5-52). A change to $2.5 \mathrm{ppm} \mathrm{O}_{2}$ at 1,321 hours increased the growth rate to $6.1 \times 10^{-7} \mathrm{~mm} / \mathrm{s}$, and introducing a 9,000s hold at $\mathrm{K}_{\max }$ did not affect the growth rate consequentially. As seen in Figure 5-53, a change to constant $\mathrm{K}$ (no cycling) was made at 1,537 hours, and the resultant growth rate was $3.2 \times 10^{-7}$ $\mathrm{mm} / \mathrm{s}$. Changing to $10 \mathrm{ppb}$ sulfate at 1,561 hours had no discernible effect on the growth rate.

At 1,700-1,800 hours (Figure 5-53), there were problems with the micropump that circulates water within the water supply system, and these affected the inlet conductivity measurement. This led to two periods in which the sulfate level increased because its level is controlled by the inlet conductivity measurement. The periods of elevated conductivity increased the growth rate, but it quickly returned to the prior rate when the water chemistry returned to normal (10 ppb sulfate). The peak outlet conductivity at $\sim 1,700$ hours was $\sim 20 \mu \mathrm{S} / \mathrm{cm}$ (which persisted for $\sim 10$ hours); this corresponds roughly to $2,000 \mathrm{ppb}$ sulfate (chromate also contributed to the outlet conductivity). At $\sim 1,770$ hours, a plateau (which persisted for $\sim 24$ hours) of $\sim 20 \mu \mathrm{S} / \mathrm{cm}$ was also observed. In both cases, the pump was fixed, and this quickly resolved the problem.

A change to pure water was made at 1,822 hours (Figure 5-54Figure 5-53), and to constant $\mathrm{K}$ (no cycling) at 1,844 hours, and the steady-state growth rate was $1.6 \times 10^{-7} \mathrm{~mm} / \mathrm{s}$. The change to $75 \mathrm{ppb} \mathrm{H}_{2}$ was made at 1,922 hours, and the growth rate dropped by $8 X$. Gentle cycling at 2,061 hours increased the growth rate a bit. A return to $2.5 \mathrm{ppm} \mathrm{O}_{2}$ at 2,157 hours produced an increase in growth rate (Figure 
5-55), and the growth rate changed very little as the loading conditions were shifted to constant K (no cycling) at 2,540 hours. The growth rate increased slightly when gentle cyclic loading was again introduced at 2,854 hours, but the higher growth rate was sustained after returning to constant $\mathrm{K}$ conditions at 2,996 hours.

Changing to $75 \mathrm{ppb} \mathrm{H}_{2}$ at 3,071 hours dramatically reduced the growth rate to $\sim 0 \mathrm{~mm} / \mathrm{s}$ (Figure 5-55). Gentle cycling was introduced at 3,356 hours (Figure 5-56), which produced a small offset in crack length, but little long-term effect on growth rate. Changing to $2.5 \mathrm{ppm} \mathrm{O}_{2}$ at 3,549 hours had no effect for $\sim 100$ hours, when a brief change from the 9,000 s hold to a 3,000s hold at $K_{\max }$ was made, and the growth rate increased to $1.8 \times 10^{-7} \mathrm{~mm} / \mathrm{s}$. This growth rate was sustained as the loading condition changed to constant K (no cycling).

At 3,860 hours, the test was ended. Figure 5-57 is a macro photograph of the fracture surface. The average intergranular crack depth on the fracture surface is about $62 \%$ higher than was indicated by DCPD. The crack length, CGR, and $\mathrm{K}$ data were corrected in all plots. The post-test corrected $\mathrm{K}$ values were higher than intended during testing because the error was moderately large and - equally important - the amount of total crack extension was moderately large. Table 5-6 is a summary of all of the CGRs and related conditions under periods of constant applied $\mathrm{K}$ for this test.

Table 5-6: Constant K IASCC CGR and related conditions for specimen 10A0001B03 of XM-19 irradiated to $0.29 \mathrm{dpa}$; blue highlights indicate higher confidence data.

\begin{tabular}{|c|c|c|c|c|c|c|c|}
\hline $\begin{array}{c}\text { Test } \\
\text { Hours }\end{array}$ & $\begin{array}{c}\mathbf{K} \\
(\mathbf{M P a V m})\end{array}$ & Chemistry & Sulfate & $\begin{array}{c}\text { Outlet } \\
\text { Cond } \\
(\boldsymbol{\mu} \mathbf{S} / \mathbf{c m})\end{array}$ & $\begin{array}{c}\text { Time } \\
\text { Increment } \\
(\mathbf{h r})\end{array}$ & $\begin{array}{c}\text { Growth } \\
\text { Increment } \\
(\mathbf{m m})\end{array}$ & $\begin{array}{c}\text { Average } \\
\mathbf{C G R} \\
(\mathbf{m m} / \mathbf{s})\end{array}$ \\
\hline 1537 & 33 & NWC & 0 & 0.087 & 24 & 0.028 & $3.20 \mathrm{E}-07$ \\
\hline 1561 & 33 & NWC & 10 & 0.129 & 261 & 0.362 & $3.20 \mathrm{E}-07$ \\
\hline 1844 & 33 & NWC & 0 & 0.08 & 148 & 0.059 & $1.60 \mathrm{E}-07$ \\
\hline 1992 & 33 & HWC & 0 & 0.059 & 69 & 0.015 & $2.00 \mathrm{E}-08$ \\
\hline 2540 & 35 & NWC & 0 & 0.081 & 314 & 0.193 & $1.80 \mathrm{E}-07$ \\
\hline 2996 & 35 & NWC & 0 & 0.075 & 79 & 0.049 & $2.20 \mathrm{E}-07$ \\
\hline 3076 & 35 & HWC & 0 & 0.059 & 280 & 0.000 & $1.00 \mathrm{E}-09$ \\
\hline 3717 & 35 & NWC & 0 & 0.083 & 143 & 0.092 & $1.80 \mathrm{E}-07$ \\
\hline
\end{tabular}




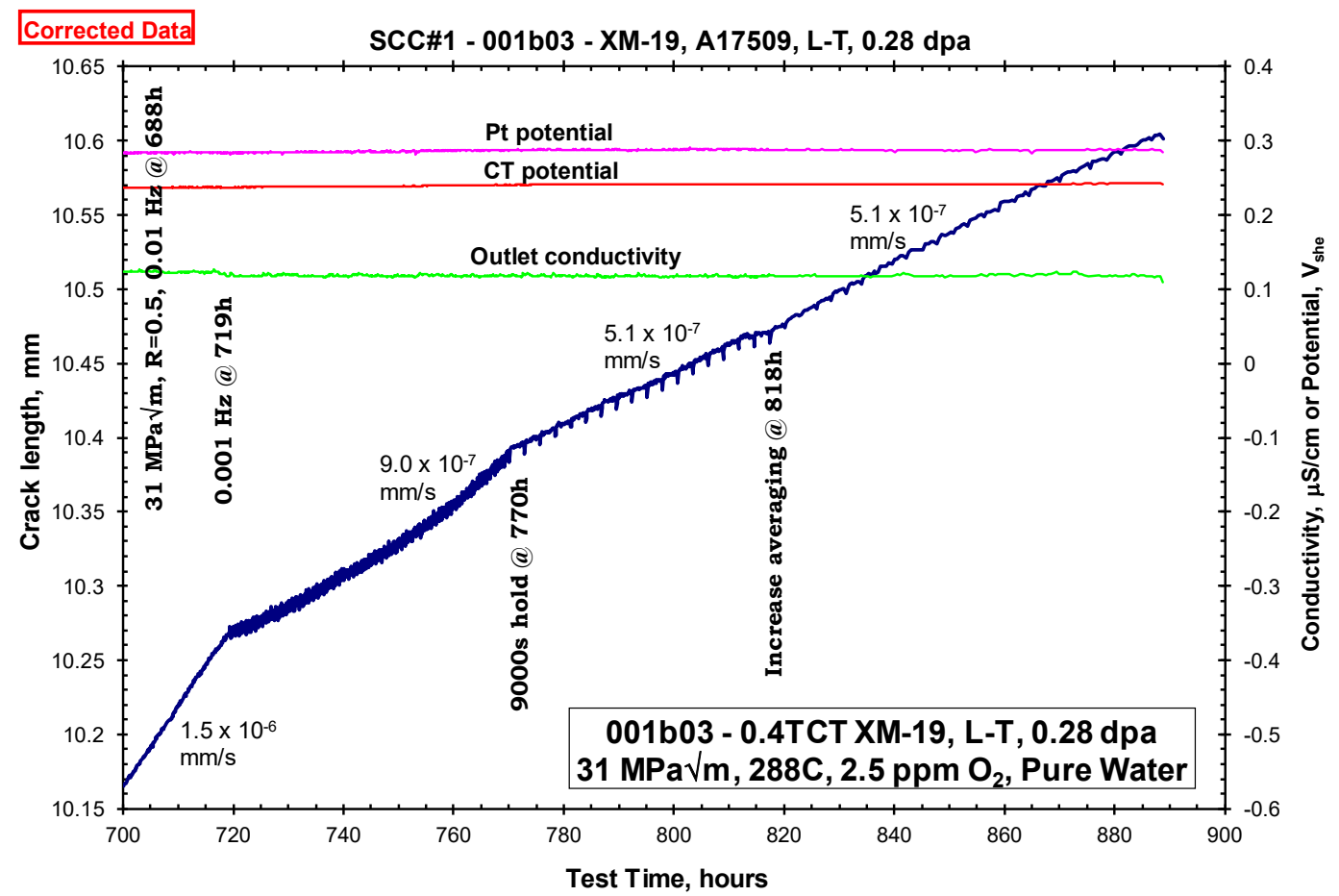

Figure 5-51: Transition to intergranular fracture for IASCC test of specimen 10A0001B03 of XM19 irradiated to 0.29 dpa.

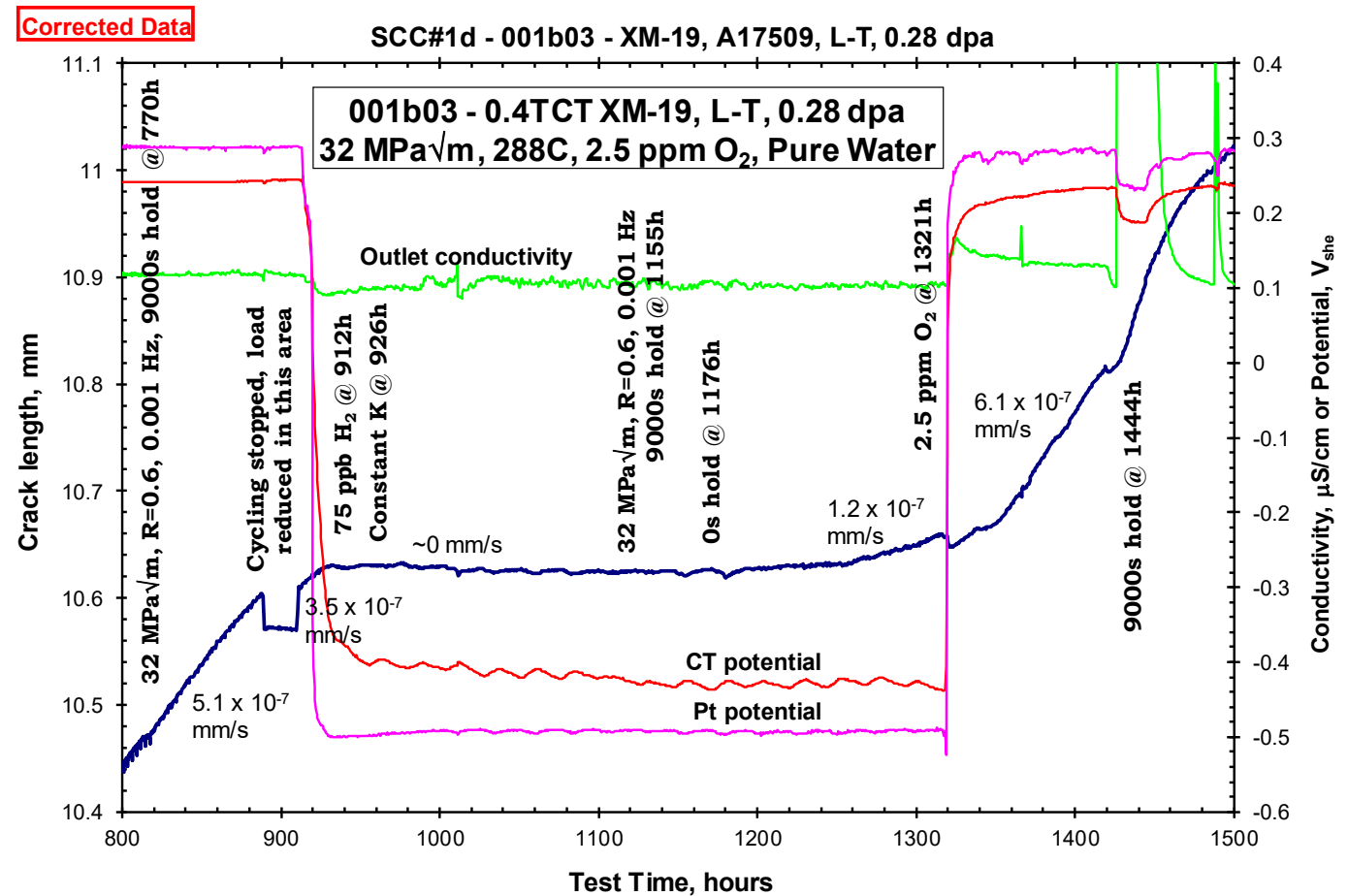

Figure 5-52: IASCC CGR response under constant $K$ and $H W C$ and NWC with periods of gentle cycling for specimen 10A0001B03 of XM-19 irradiated to $0.29 \mathrm{dpa}$. 


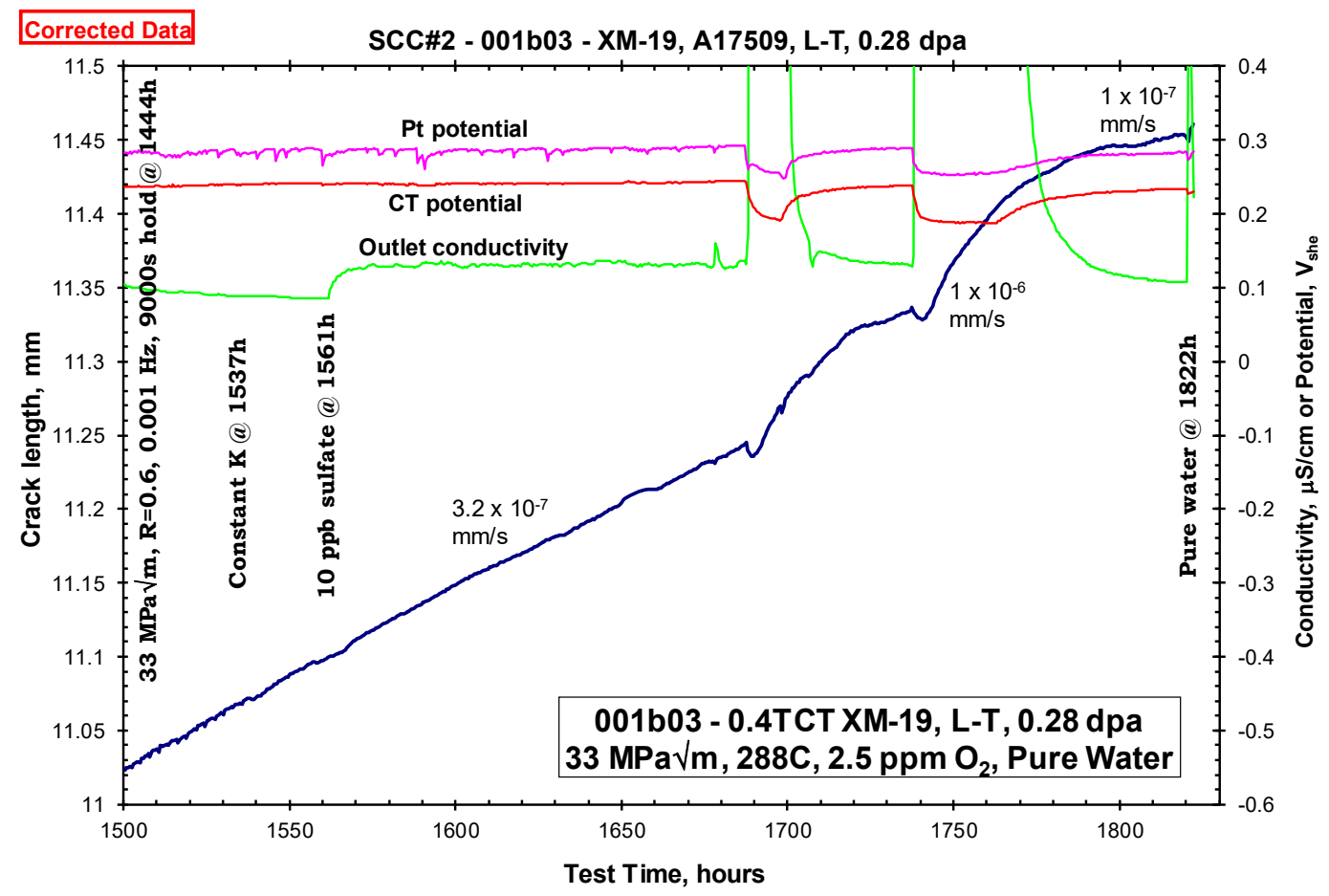

Figure 5-53: IASCC CGR response under constant $K$ and NWC with sulfate addition for specimen $10 \mathrm{A0001B03}$ of XM-19 irradiated to 0.29 dpa.

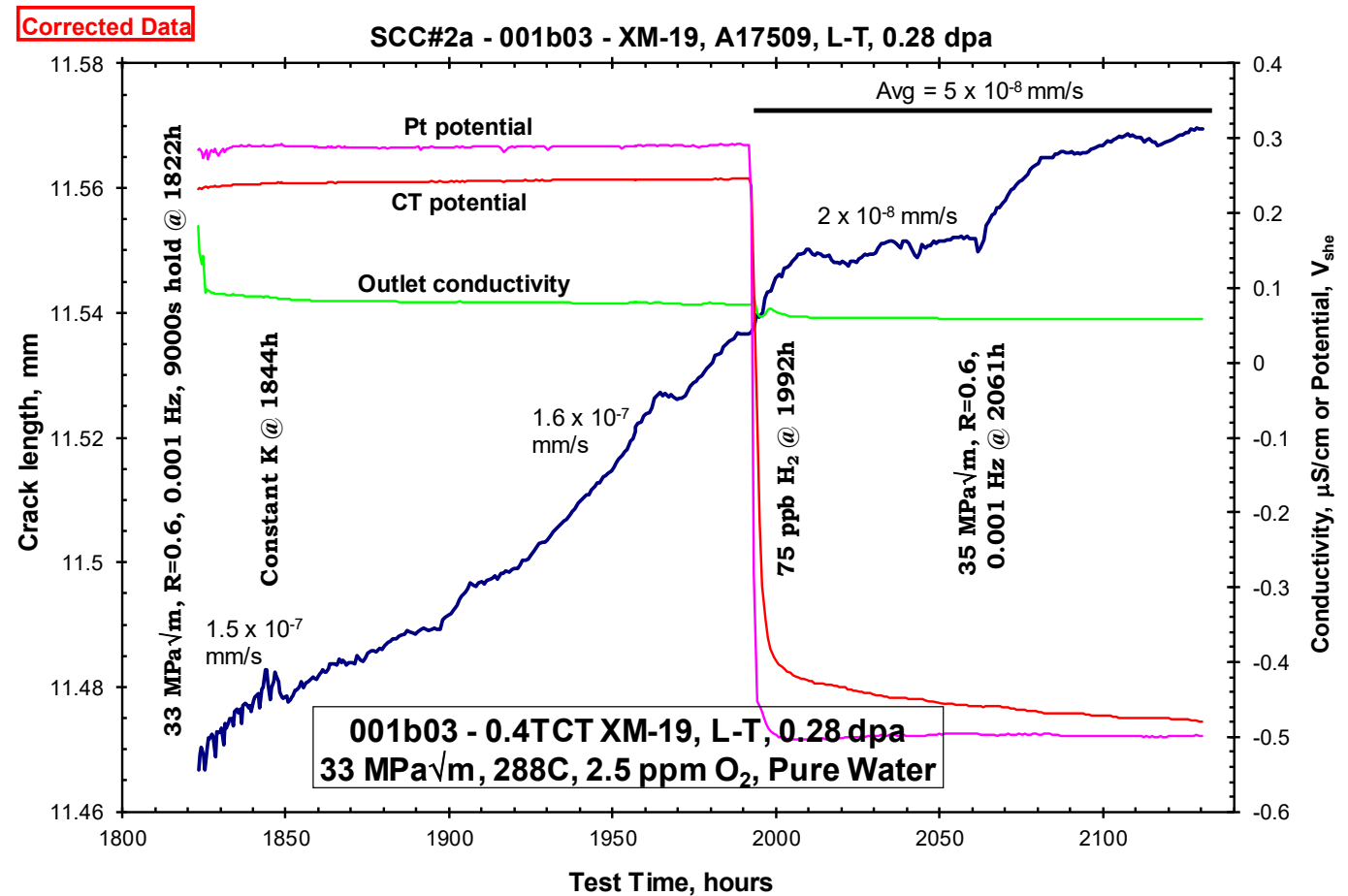

Figure 5-54: IASCC CGR steady-state response under sustained constant $K$, and NWC and HWC for specimen 10A0001B03 of XM-19 irradiated to 0.29 dpa. 


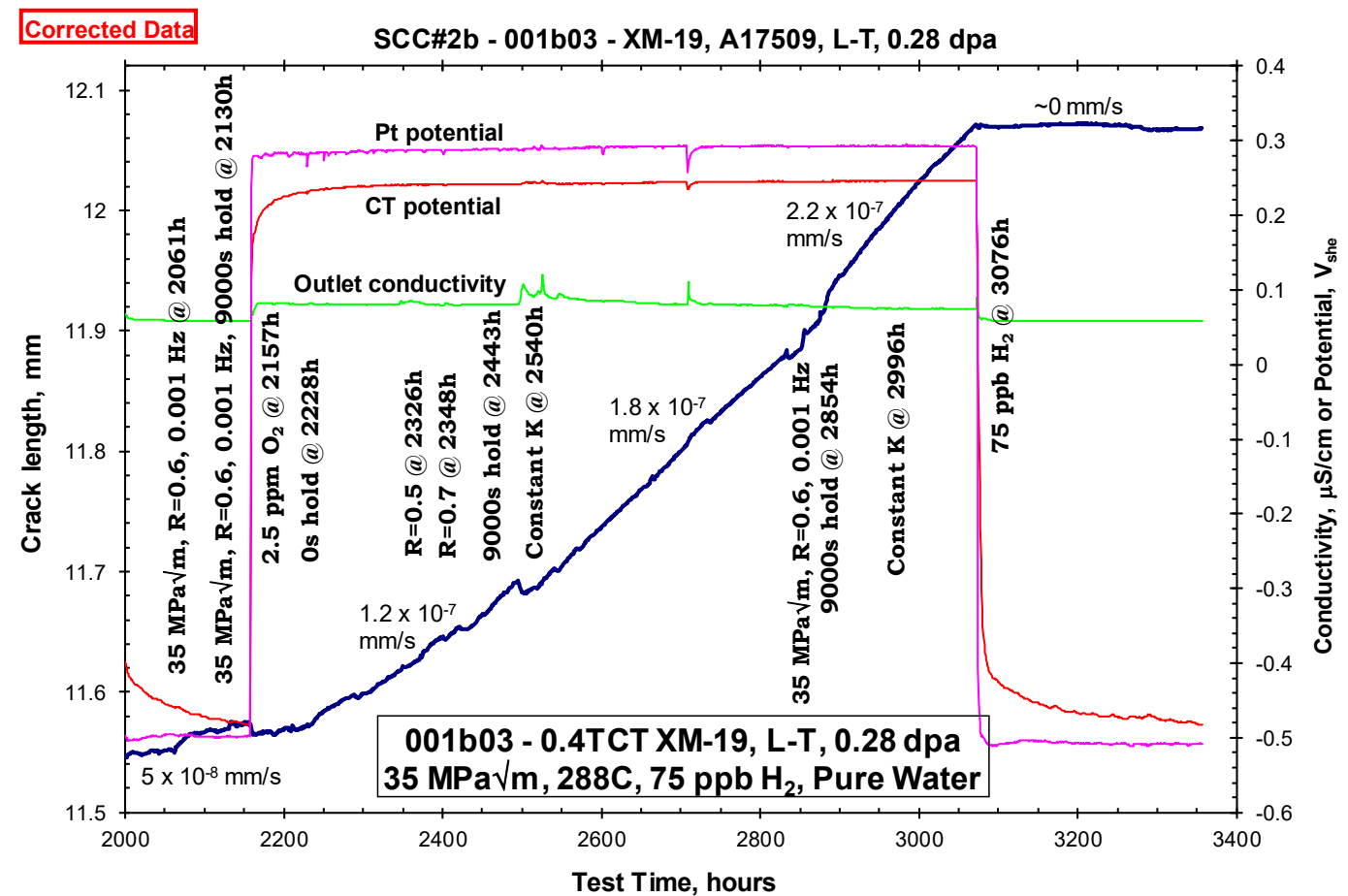

Figure 5-55: IASCC CGR response under gentle cycling followed by constant $K$ under NWC for specimen 10A0001B03 of XM-19 irradiated to 0.29 dpa.

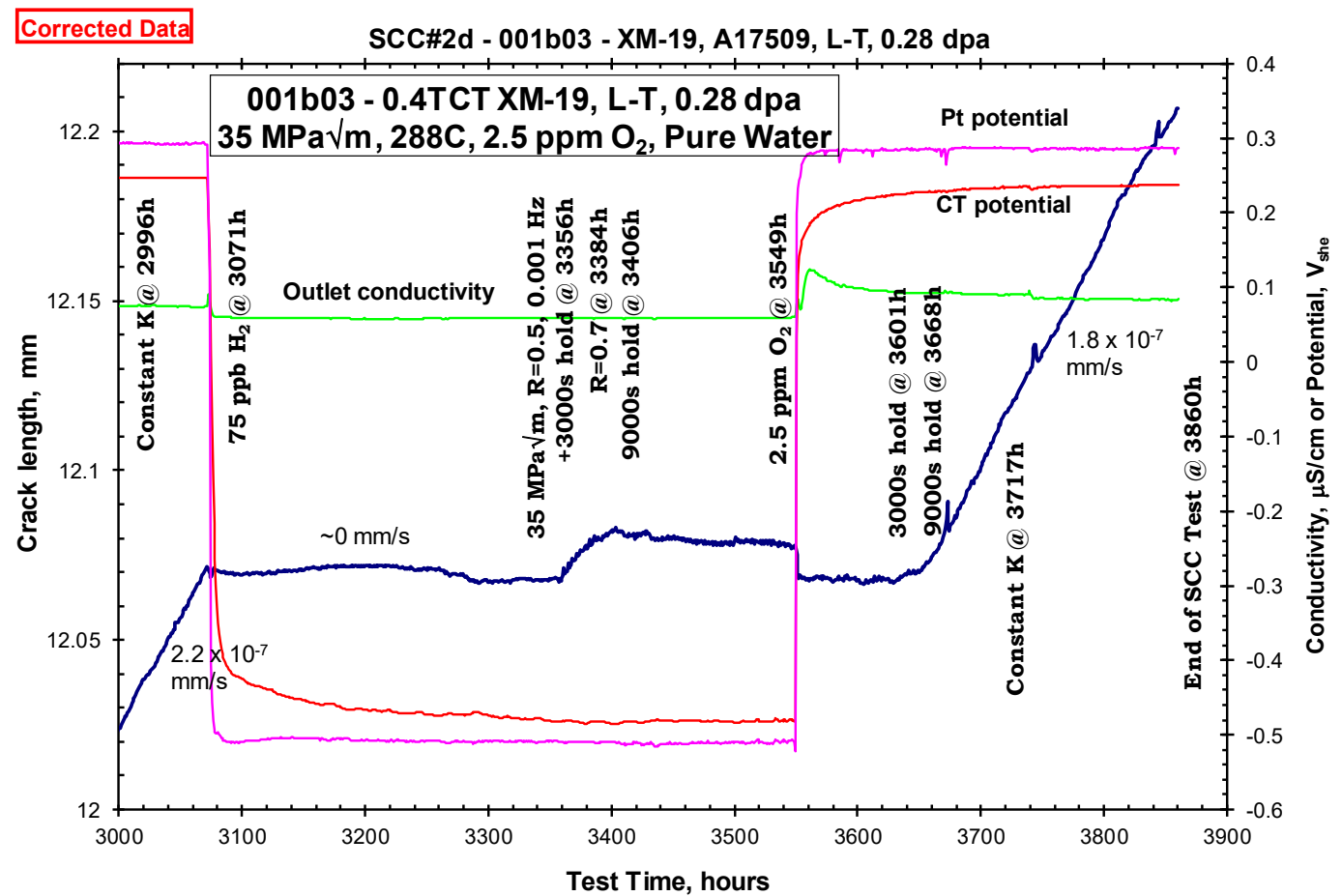

Figure 5-56: IASCC CGR response under constant $K$ for return to $H W C$, and final steps for specimen 10A0001B03 of XM-19 irradiated to 0.29 dpa. 


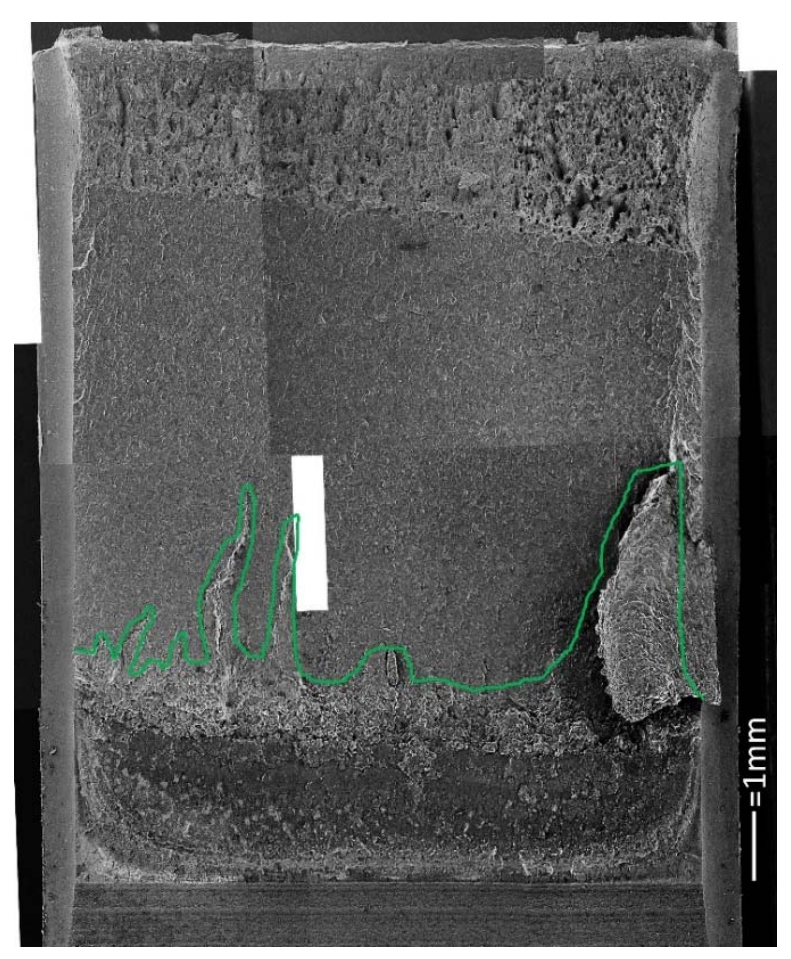

Figure 5-57: SEM micrograph showing fracture surface for specimen 10A0001B03 of XM-19 irradiated to 0.29 dpa.

\subsubsection{High Fluence XM-19 IASCC CGR Tests}

As shown in Table 3-7, specimens 10A0001B07 and 10A0001D02 were irradiated over two ATR cycles to fast neutron damage levels of approximately 1.32 and $1.44 \mathrm{dpa}$, respectively. Estimated irradiation temperatures for 10A0001B07 over the first and second cycles were $287^{\circ} \mathrm{C}$ (first cycle) and $257^{\circ} \mathrm{C}$ (second cycle) on average, with short-duration maximums of $295^{\circ} \mathrm{C}$ (first cycle) and $259^{\circ} \mathrm{C}$ (second cycle). Estimated irradiation temperatures for 10A0001D02 over the first and second cycles were $329^{\circ} \mathrm{C}$ (first cycle) and $262{ }^{\circ} \mathrm{C}$ (second cycle) on average, with short-duration maximums of $341^{\circ} \mathrm{C}$ (first cycle) and $265^{\circ} \mathrm{C}$ (second cycle). The details of these two IASCC tests follow; in all plots, the blue line tracks crack length vs time (CGR).

\subsubsection{Specimen 10A0001B07 (1.32 dpa) XM-19 IASCC test}

Figure 5-58 provides an overview of the response of specimen 10A0001B07, which was started in $288^{\circ} \mathrm{C}$ pure water with $2.5 \mathrm{ppm} \mathrm{O}_{2}$. Figure 5-59 shows the in-situ fatigue pre-cracking response. In each graph, the label in the outlined box represents the starting condition for that plot. Some graphs show additional $\mathrm{K}$ values, which represent an evolution in $\mathrm{K}$ resulting from the post-test correction, although intentional changes in $\mathrm{K}$ were also made. 


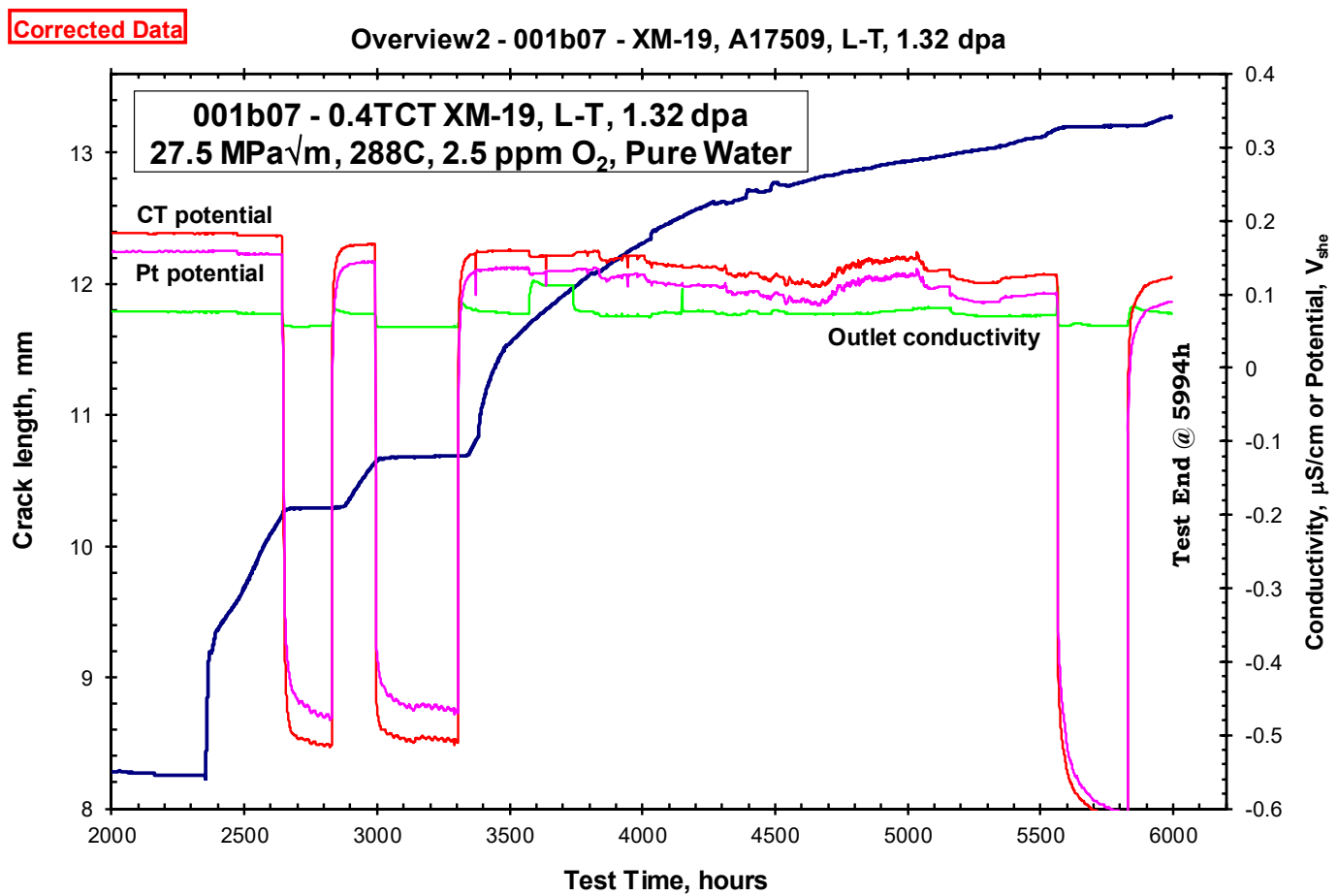

Figure 5-58: IASCC CGR test overview for specimen 10A0001B07 of XM-19 irradiated to 1.32 dpa.

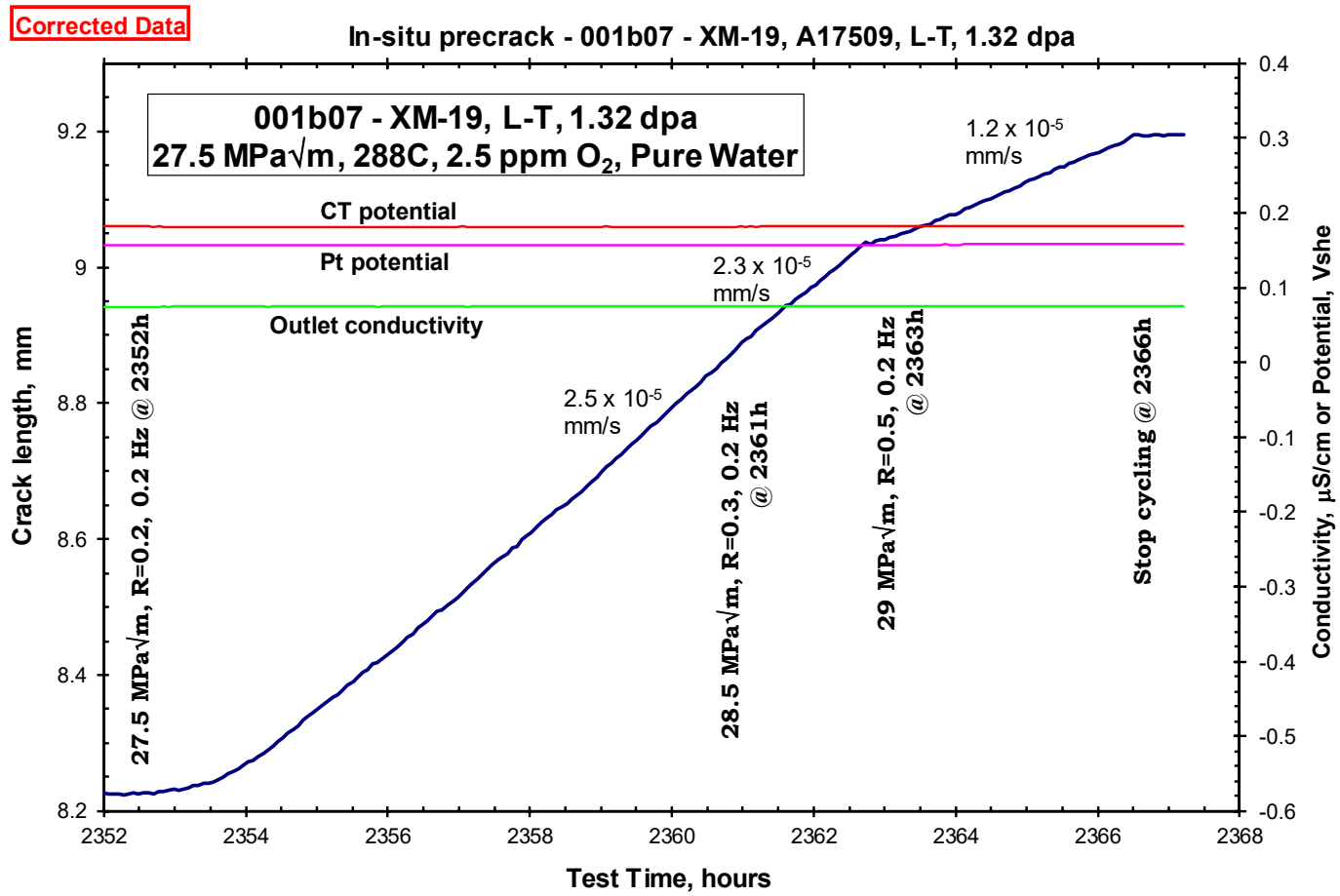

Figure 5-59: In-situ pre-fatigue response for specimen 10A0001B07 of XM-19 irradiated to 1.32 dpa.

Intergranular transitioning was initiated at 2,366 hours at $\mathrm{K}_{\max }=29 \mathrm{MPa} \sqrt{\mathrm{m}}$, loading ratio $\mathrm{R}=0.6$, and frequencies of $0.01 \mathrm{~Hz}$ followed by $0.001 \mathrm{~Hz}$, then with a 3,500s, 9,500s, and 27,300s hold at $\mathrm{K}_{\max }$ (Figure 5-60). At 2,563 hours, a change to constant K (no cycling) conditions was made. The growth rate 
remained constant at roughly $10^{-6} \mathrm{~mm} / \mathrm{s}$. At 2,640 hours, a change was made to $95 \mathrm{ppb} \mathrm{H}_{2}$ (Figure 5-61), and the growth rate dropped by about $1000 \mathrm{X}$.

At 2,830 hours, a return to $2.5 \mathrm{ppm} \mathrm{O}_{2}$ was made, and the growth rate increased to close to the prior value in the same environment. At 2,994 hours, a change was again made to $95 \mathrm{ppb} \mathrm{H}_{2}$ (Figure 5-62), and the growth rate dropped by about $51 \mathrm{X}$. At 3,306 hours, the change to $2.5 \mathrm{ppm} \mathrm{O}_{2}$ again produced an increase in growth rate close to the prior value in the same environment.

Gentle cycling was initiated at 2,563 hours, and a transient growth rate was observed that slowed over time to the constant $\mathrm{K}$ rate of about $1.2 \times 10^{-6} \mathrm{~mm} / \mathrm{s}$ (Figure 5-63). This transient is interpreted as a crack front that started uneven and straightened during cycling. Projecting the steady-state growth rate back to the start of cycling suggests that there was about $0.3 \mathrm{~mm}$ of unevenness before cycling.

At 3,480 hours, loading was switched to constant $\mathrm{K}$ (no cycling), and the growth rate decreased by about $2 \mathrm{X}$ to $5.4 \times 10^{-7} \mathrm{~mm} / \mathrm{s}$. Adding then removing sulfate at 3,572 and 3,815 hours, respectively, produced no change in growth rate. At 3,979 hours, gentle cycling and -dK/da (falling K) were used to reduce $\mathrm{K}$ from 37 to $23 \mathrm{MPa} \sqrt{\mathrm{m}}$ (Figure 5-64 and Figure 5-65). Figure 5-66 shows the $\mathrm{K}$ trajectory during the $-\mathrm{dK} /$ da period, and also shows the shallow increase in $\mathrm{K}$ before and after the $-\mathrm{dK} / \mathrm{da}$ period that results from the post-test correction. During this time, the growth rate changed from 2.7 to $0.87 \times 10^{-7} \mathrm{~mm} / \mathrm{s}$, which reflects a $\mathrm{K}$ dependency of $\sim \mathrm{K}^{2.5}$.

A return to constant $\mathrm{K}$ conditions at 5,213 hours (Figure 5-67 and Figure 5-68) produced an average growth rate of $1.2 \times 10^{-7} \mathrm{~mm} / \mathrm{s}$. Toward the end of this period, the growth rate was about $3 \times 10^{-7} \mathrm{~mm} / \mathrm{s}$. At 5,563 hours, a change was made to $95 \mathrm{ppb} \mathrm{H}_{2}$ (Figure 5-69), and the growth rate dropped by $30 \mathrm{X}$ to $1 \times 10^{-8} \mathrm{~mm} / \mathrm{s}$. The occasional dips in crack length vs. time are probably the result of temperature fluctuations in the ambient laboratory environment. At 5,828 hours, a return to $2.5 \mathrm{ppm} \mathrm{O}_{2}$ was made, and the growth rate increased to approximately the prior rate in the same environment.

At 5,994 hours, the test ended. Figure 5-70 is a macro photograph of the fracture surface, and Figure 5-71 shows a side view that indicates the crack had extensively branched out on one side of the specimen. The average intergranular crack depth on the fracture surface is about $30 \%$ greater than was indicated by DCPD. The crack length, CGR, and $\mathrm{K}$ data were corrected in all plots. The post-test corrected $\mathrm{K}$ values were higher than intended during testing because the error was moderately large, although the average crack extension was moderate. Table 5-7 is a summary of all the CGRs and related conditions under periods of constant applied $\mathrm{K}$ for this test.

Table 5-7: Constant K IASCC CGR and related conditions for specimen 10A0001B07 of XM-19 irradiated to 1.32 dpa.

\begin{tabular}{|c|c|c|c|c|c|c|c|}
\hline $\begin{array}{c}\text { Test } \\
\text { Hours }\end{array}$ & $\begin{array}{c}\mathbf{K} \\
(\mathbf{M P a V m})\end{array}$ & Chemistry & Sulfate & $\begin{array}{c}\text { Outlet } \\
\text { Cond } \\
(\boldsymbol{\mu} \mathbf{S} / \mathbf{c m})\end{array}$ & $\begin{array}{c}\text { Time } \\
\text { Increment } \\
(\mathbf{h r})\end{array}$ & $\begin{array}{c}\text { Growth } \\
\text { Increment } \\
(\mathbf{m m})\end{array}$ & $\begin{array}{c}\text { Average } \\
\text { CGR } \\
(\mathbf{m m} / \mathbf{s})\end{array}$ \\
\hline 2563 & 30 & NWC & 0 & 0.074 & 77 & 0.263 & $1.00 \mathrm{E}-06$ \\
\hline 2640 & 30 & HWC & 0 & 0.058 & 190 & 0.063 & $2.00 \mathrm{E}-09$ \\
\hline 2830 & 32 & NWC & 0 & 0.073 & 164 & 0.355 & $8.20 \mathrm{E}-07$ \\
\hline 2994 & 33 & HWC & 0 & 0.056 & 312 & 0.047 & $1.60 \mathrm{E}-08$ \\
\hline 3306 & 34 & NWC & 0 & 0.078 & 76 & 0.150 & $1.00 \mathrm{E}-06$ \\
\hline 3480 & 36 & NWC & 0 & 0.074 & 92 & 0.176 & $5.40 \mathrm{E}-07$ \\
\hline 3572 & 36 & NWC & 8 & 0.113 & 243 & 0.389 & $5.40 \mathrm{E}-07$ \\
\hline 3815 & 36 & NWC & 0 & 0.07 & 164 & 0.204 & $5.40 \mathrm{E}-07$ \\
\hline 5213 & 23 & NWC & 0 & 0.072 & 350 & 0.176 & $2.00 \mathrm{E}-07$ \\
\hline 5563 & 23 & HWC & 0 & 0.057 & 265 & 0.024 & $3.00 \mathrm{E}-09$ \\
\hline
\end{tabular}




\begin{tabular}{l|l|l|l|l|l|l|l|}
5828 & 23 & NWC & 0 & 0.075 & 166 & 0.072 & $2.30 \mathrm{E}-07$ \\
\hline
\end{tabular}

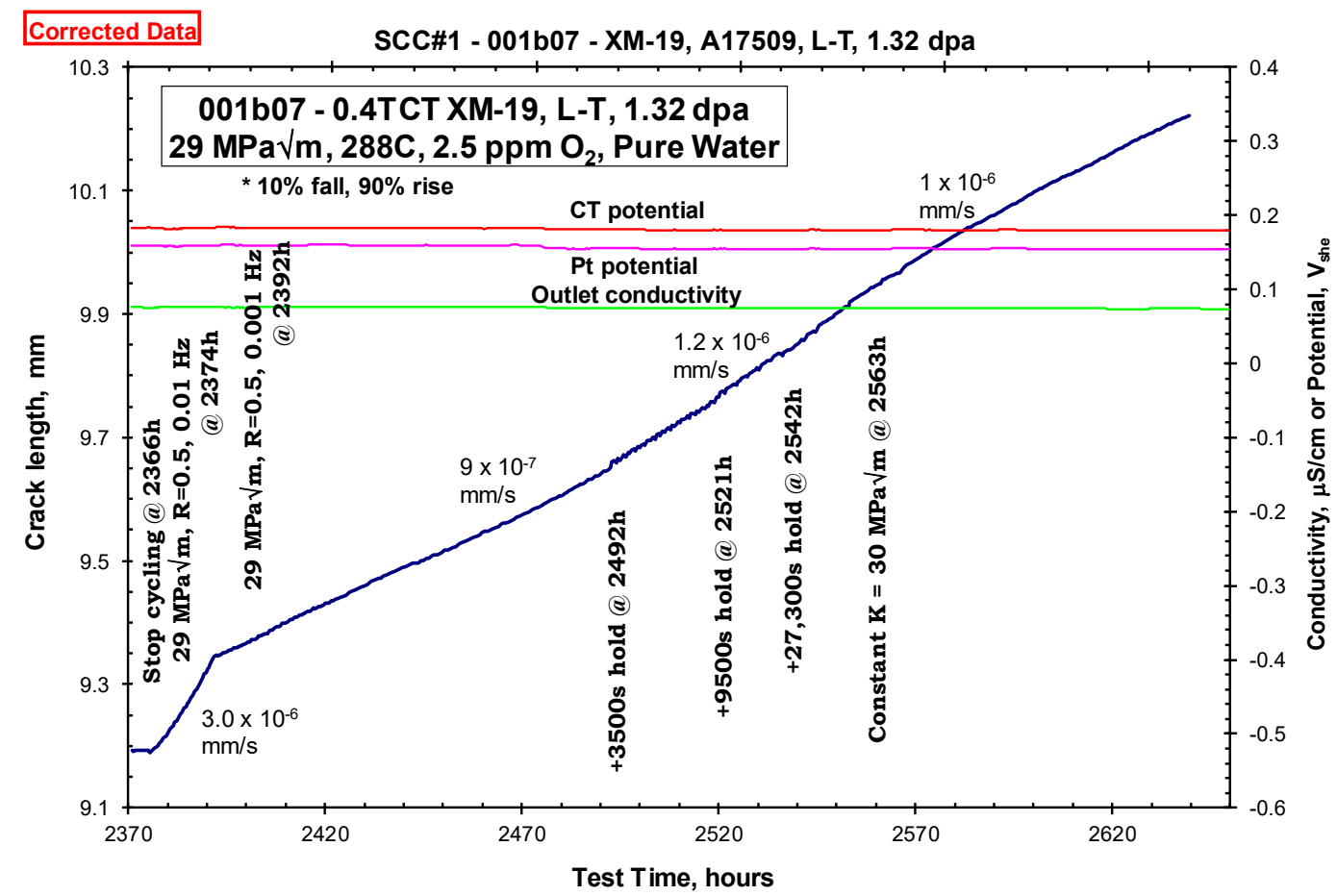

Figure 5-60: Transition to intergranular fracture for specimen $10 \mathrm{A0001 \textrm {B } 0 7}$ of XM-19 irradiated to 1.32 dpa. 


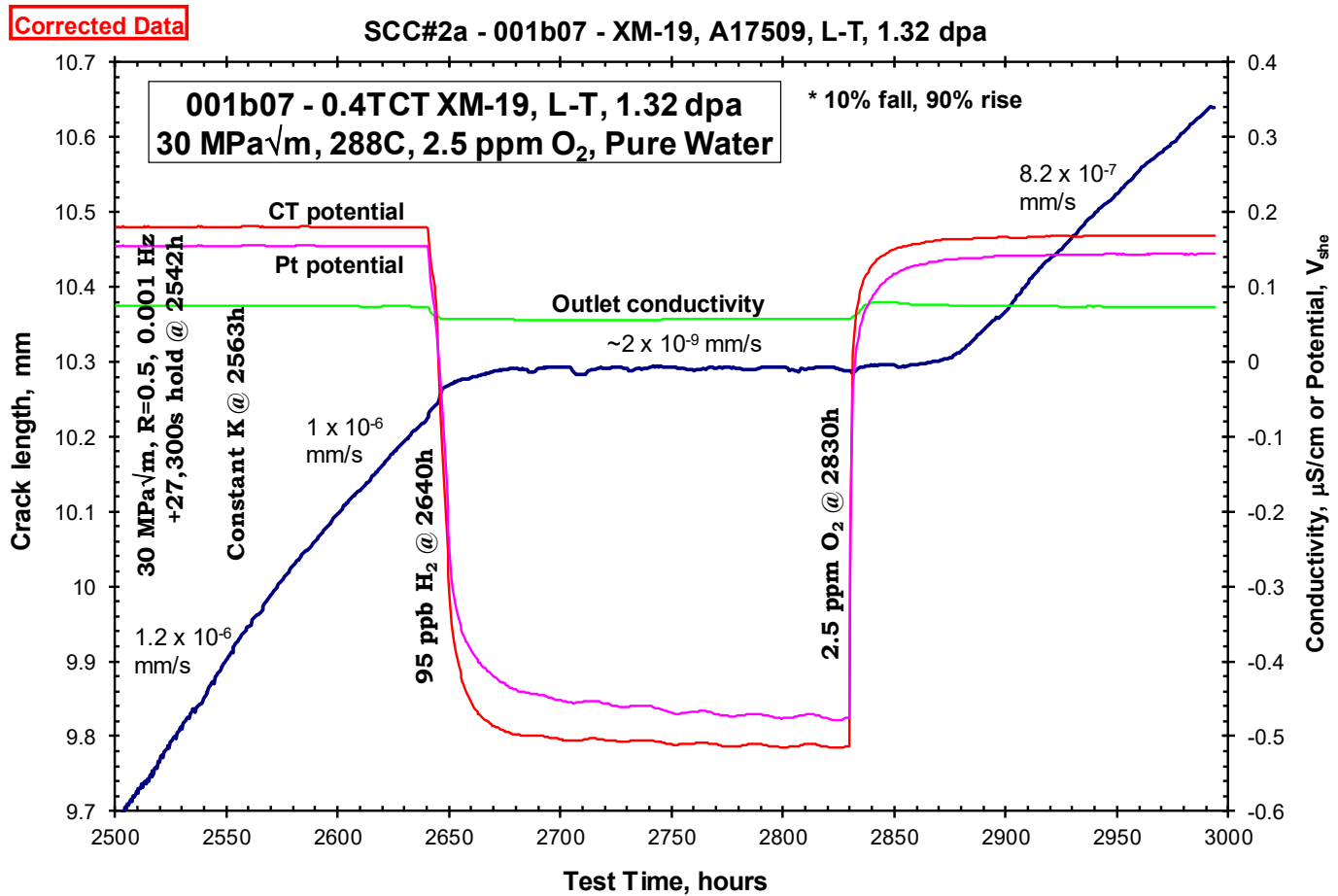

Figure 5-61: IASCC CGR response under constant $K$ and transitions to $\mathrm{HWC}$ and back to NWC for specimen 10A0001B07 of XM-19 irradiated to 1.32 dpa.

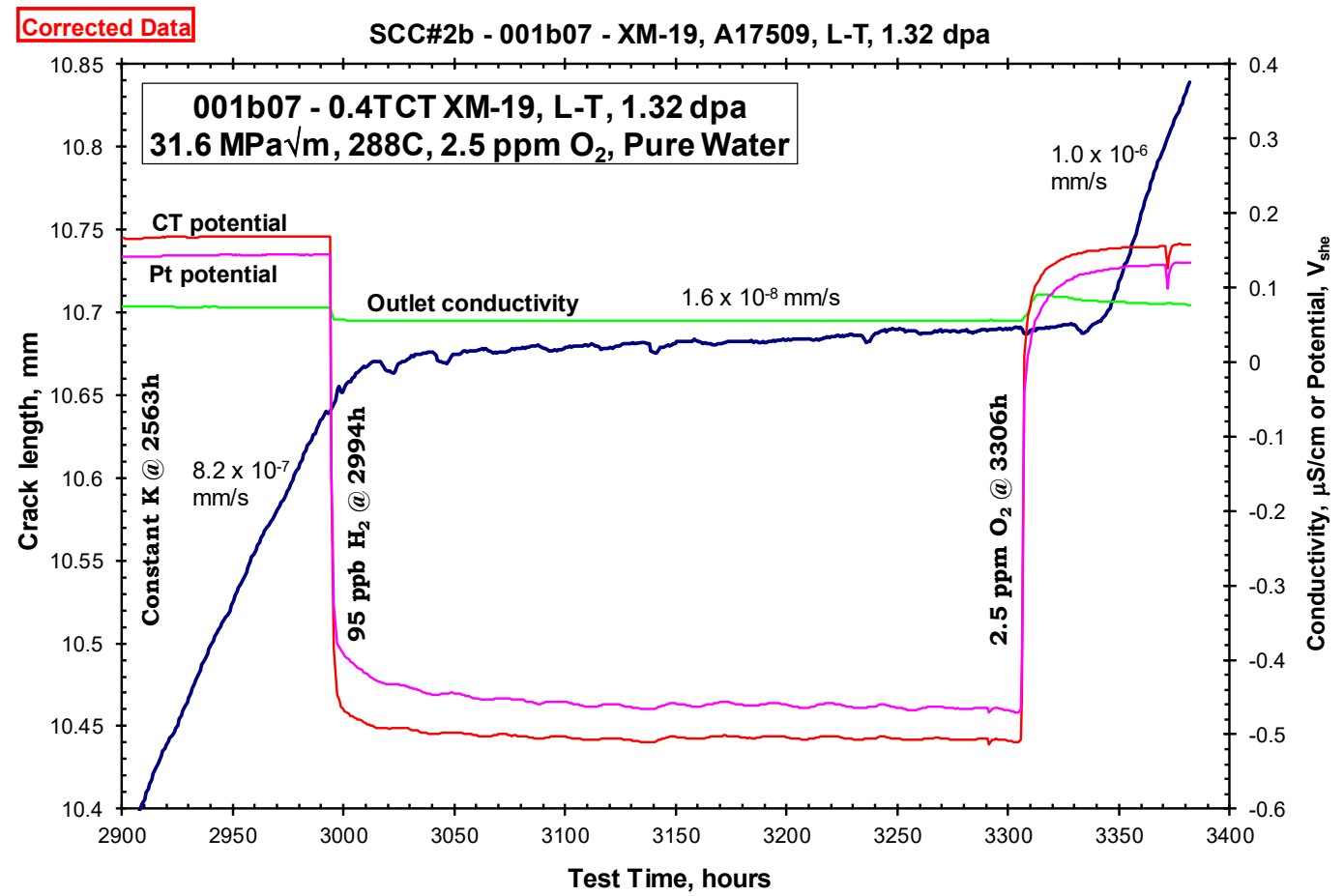

Figure 5-62: IASCC CGR response under constant $K$ with second transitions to HWC and back to NWC for specimen 10A0001B07 of XM-19 irradiated to $1.32 \mathrm{dpa}$. 


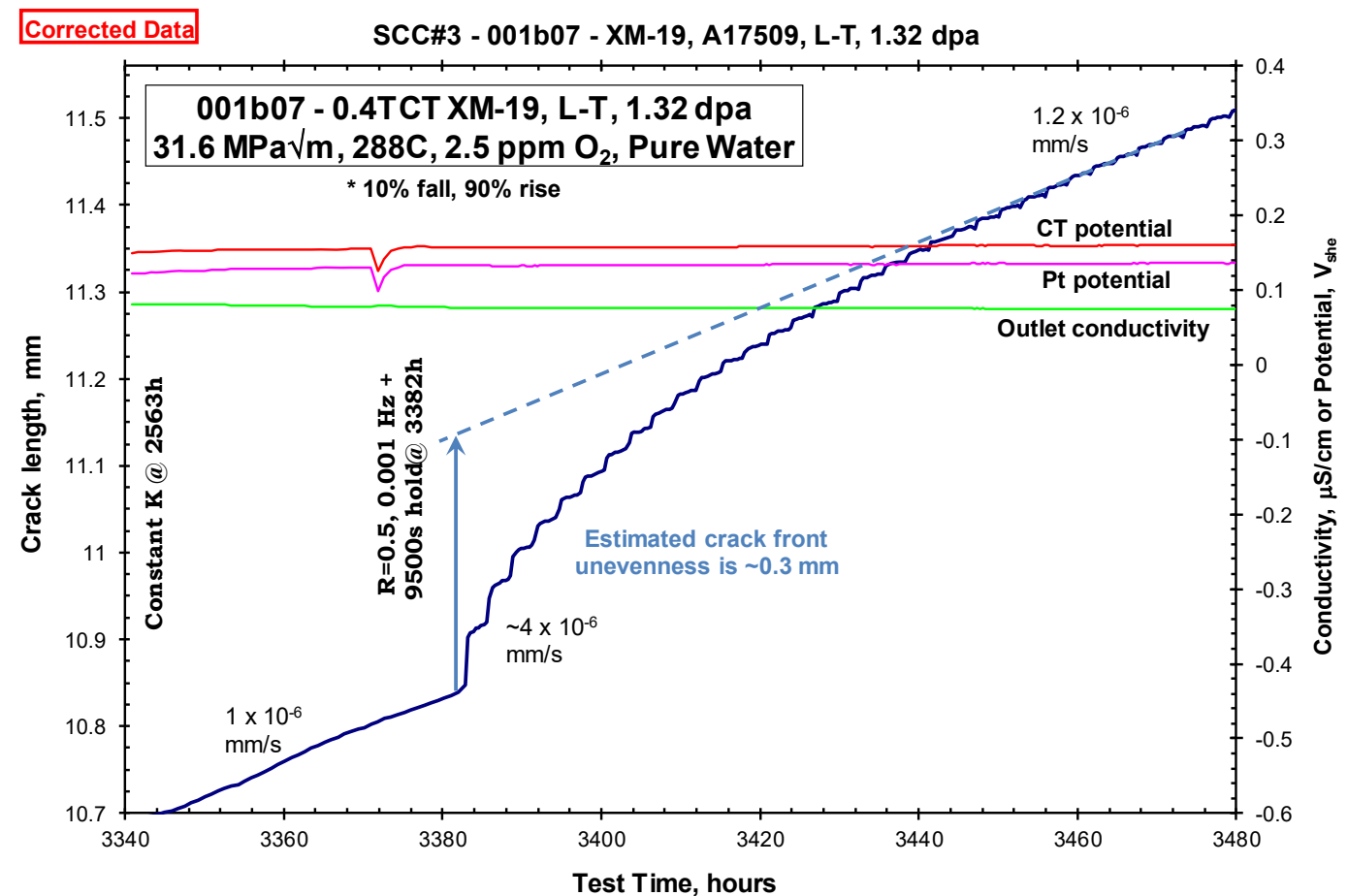

Figure 5-63: Crack front straightening for specimen 10A0001B07 of XM-19 irradiated to 1.32 dpa.

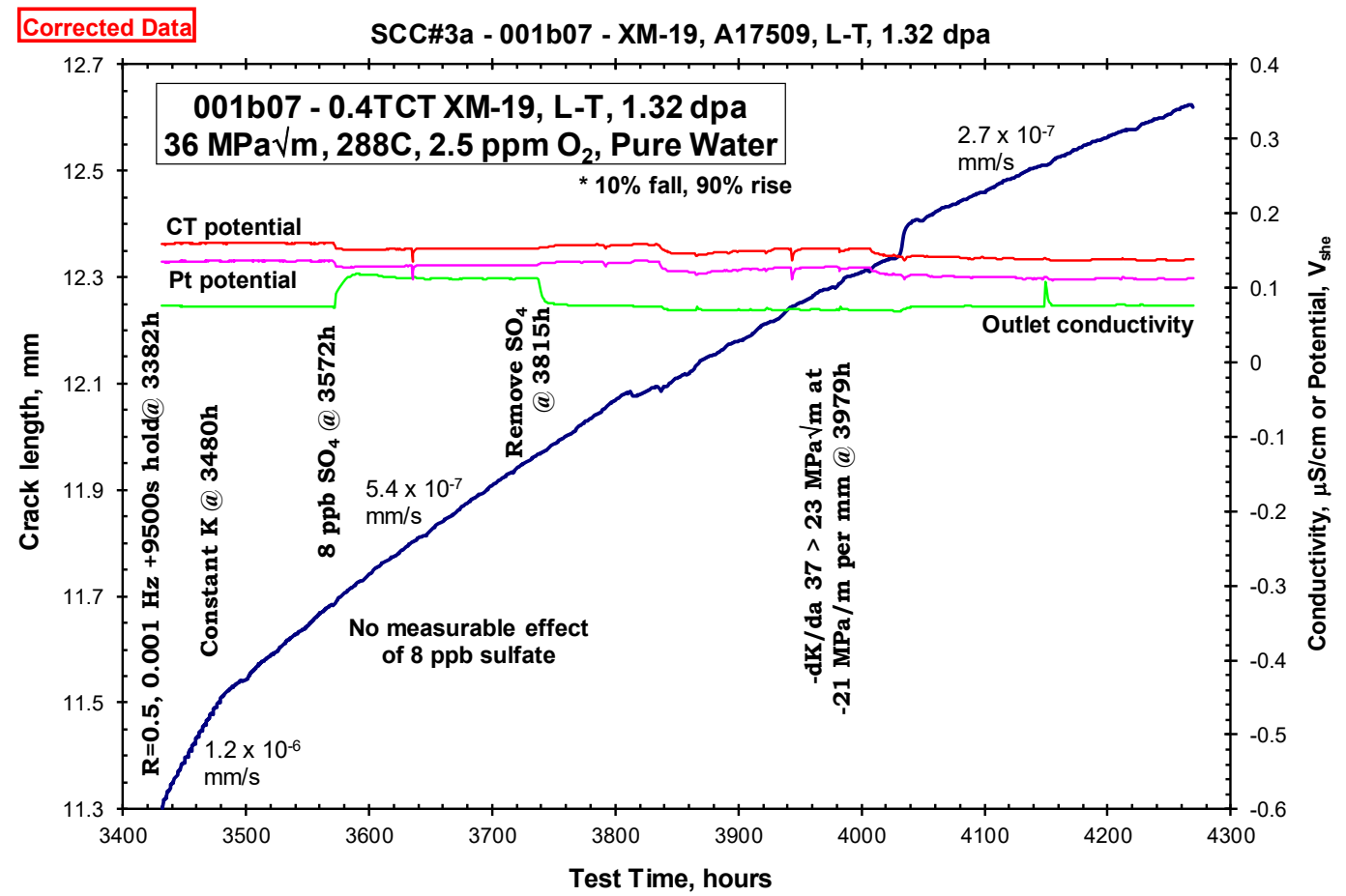

Figure 5-64: Removal of sulfate and initiation of falling $K$ for specimen 10A0001B07 of XM-19 irradiated to 1.32 dpa. 


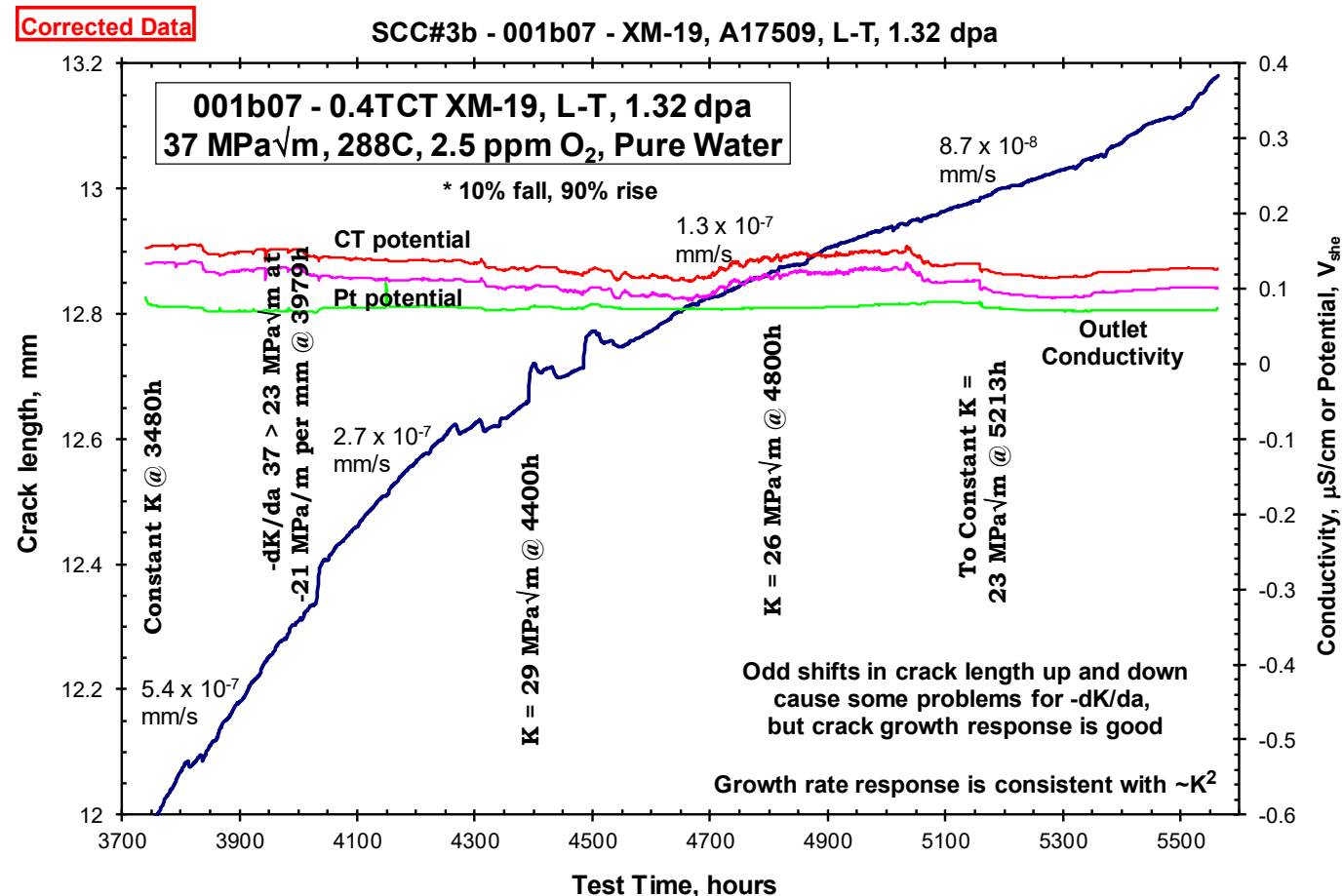

Figure 5-65: Continuation of falling $K$ and transition to constant $K$ for specimen 10A0001B07 of XM-19 irradiated to 1.32 dpa.

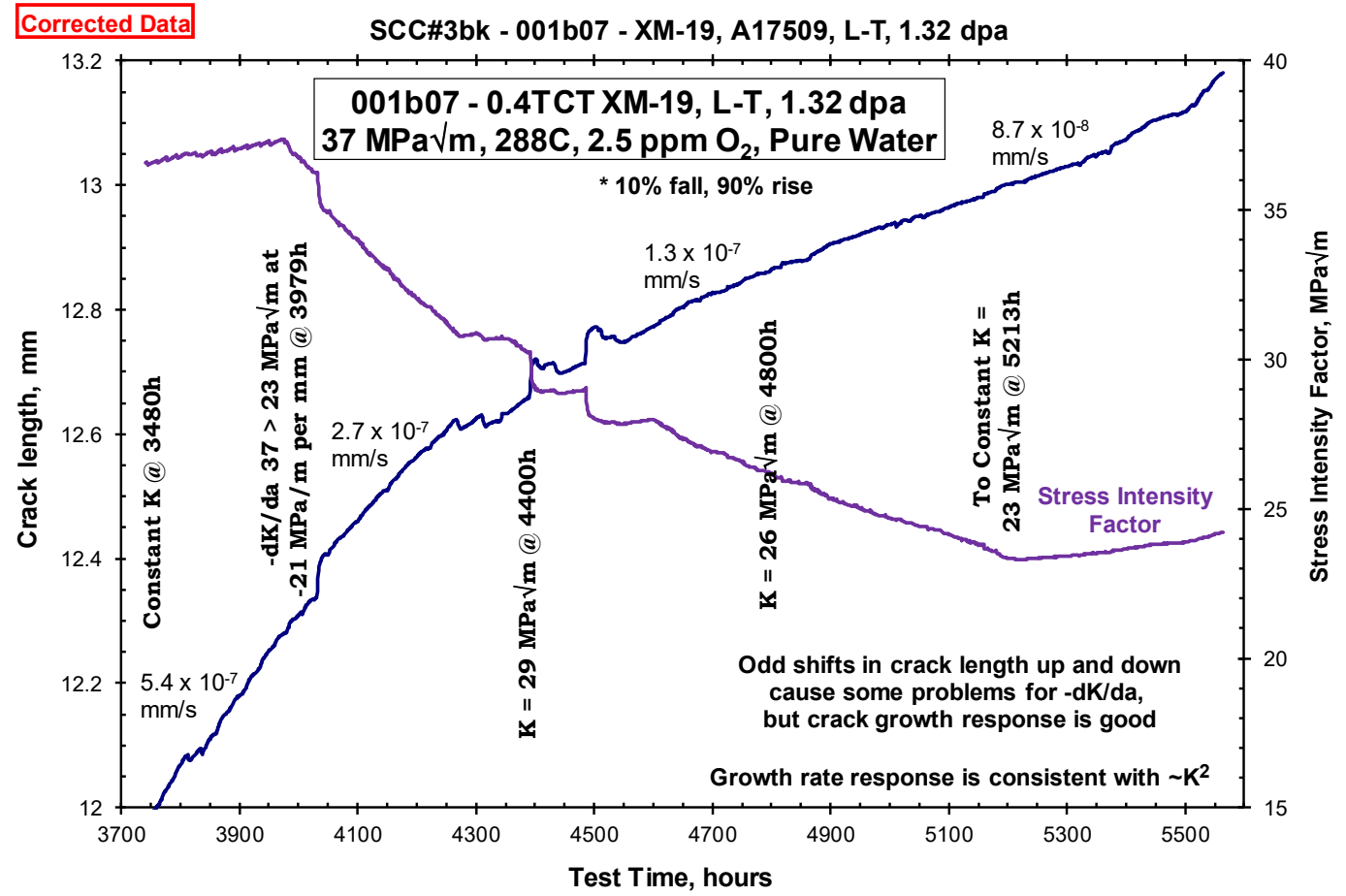

Figure 5-66: Trajectory of falling $K$ and effect on IASCC CGR for specimen 10A0001B07 of XM-19 irradiated to 1.32 dpa. 


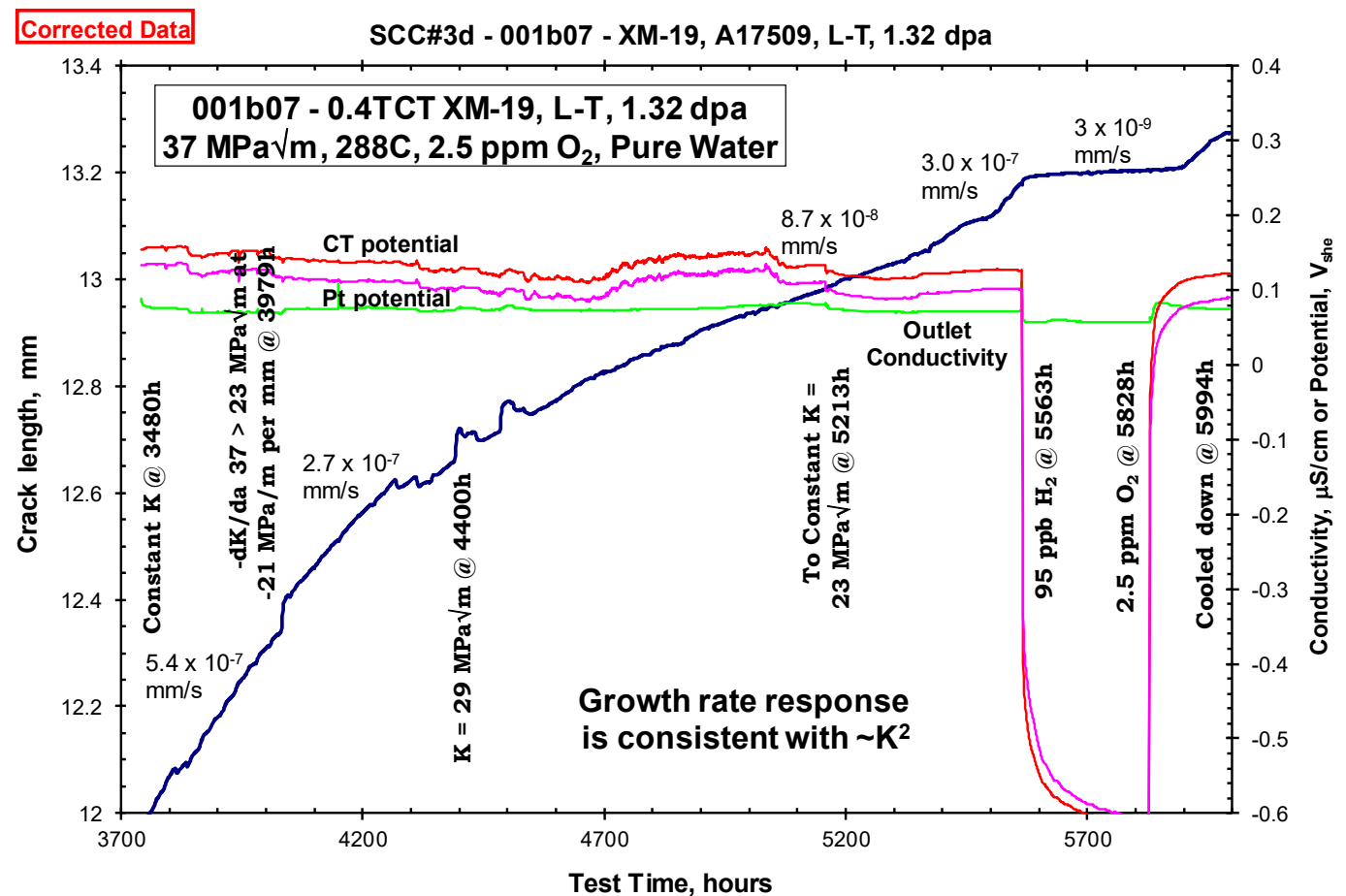

Figure 5-67: IASCC CGR response following -dK/da under NWC and HWC for specimen 10A0001B07 of XM-19 irradiated to 1.32 dpa.

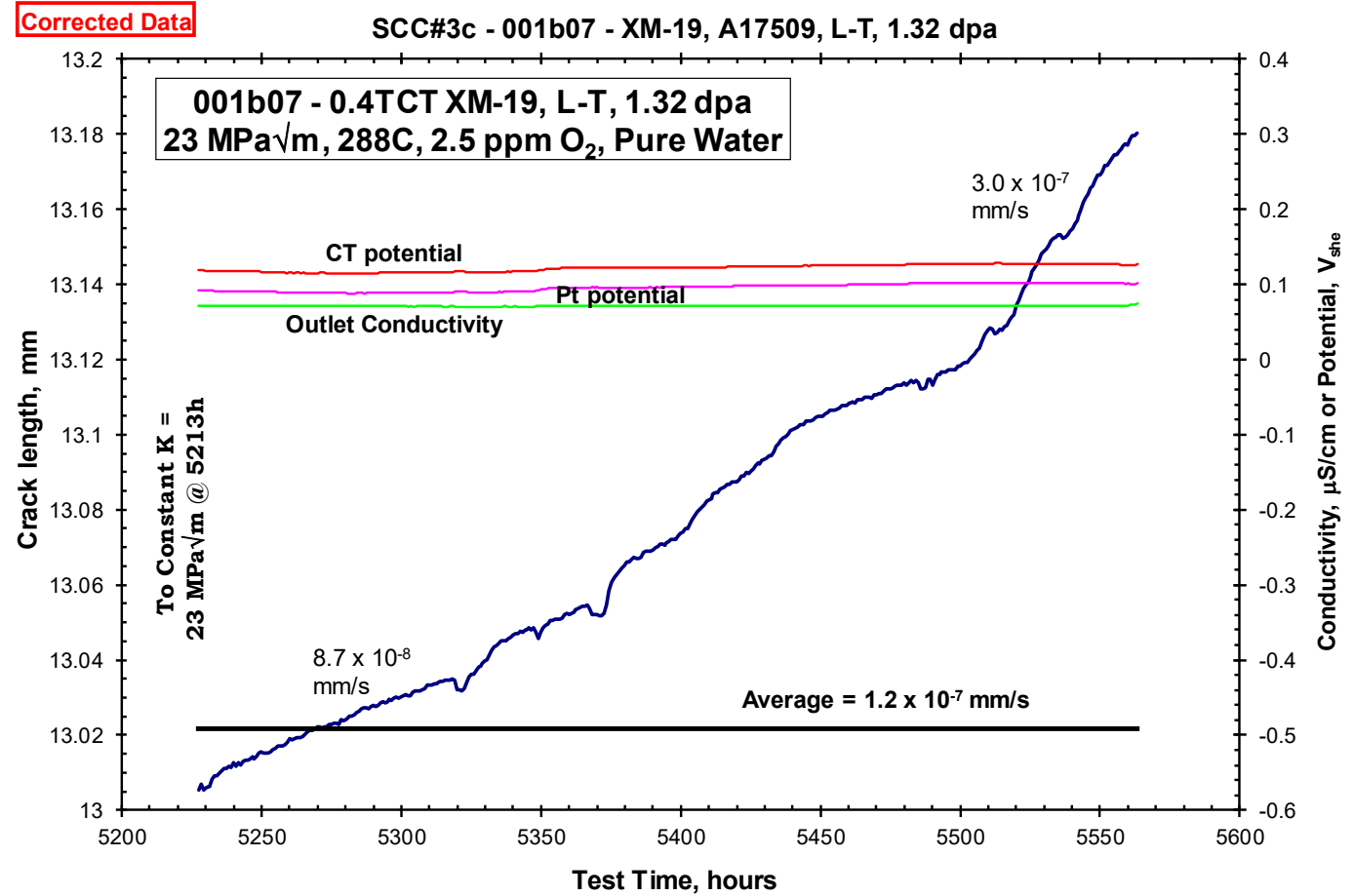

Figure 5-68: Closeup of region immediately following -dK/da for specimen 10A0001B07 of XM-19 irradiated to 1.32 dpa. 


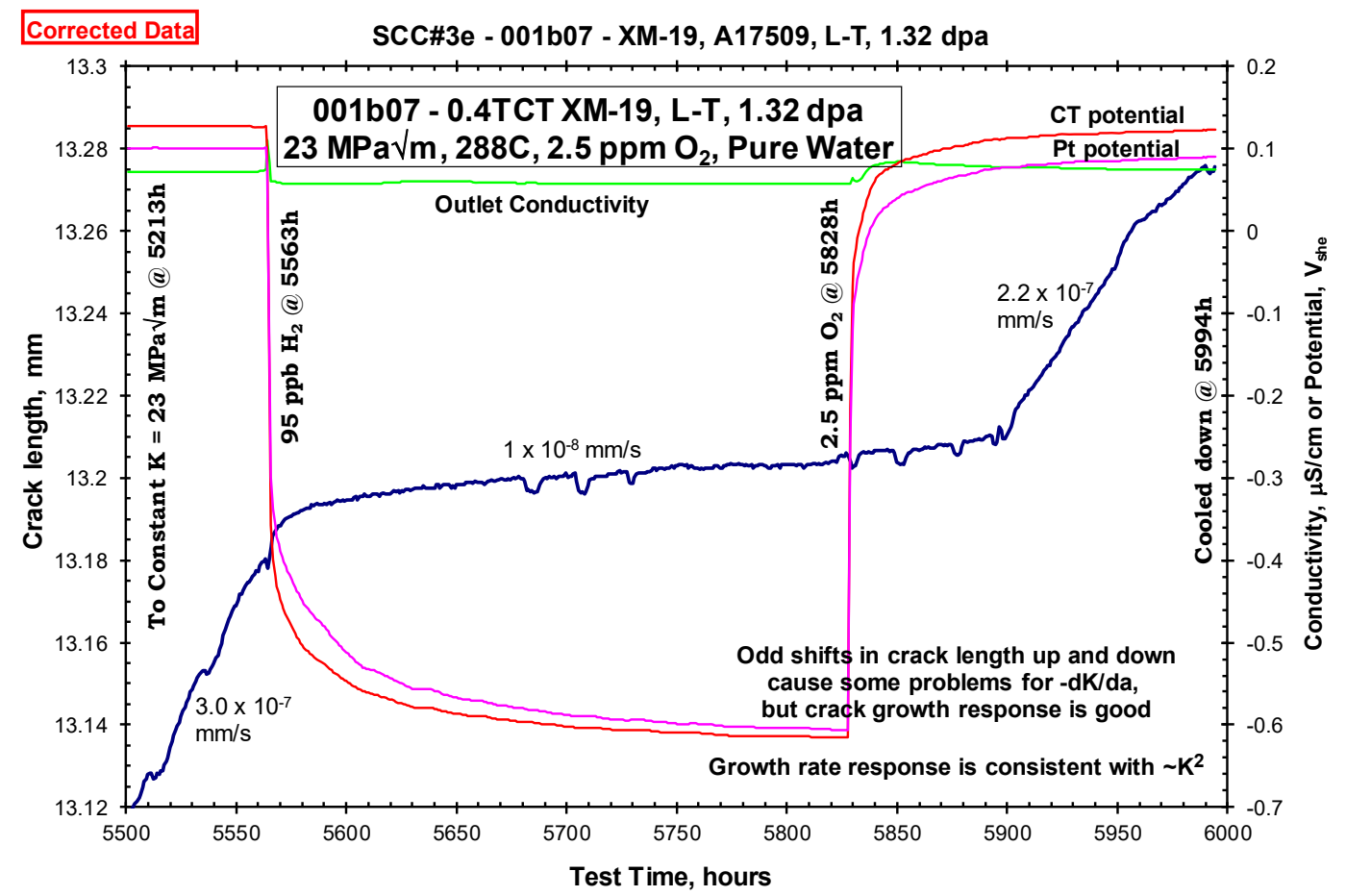

Figure 5-69: Final steps of IASCC CGR test for specimen 10A0001B07 of XM-19 irradiated to 1.32 dpa.

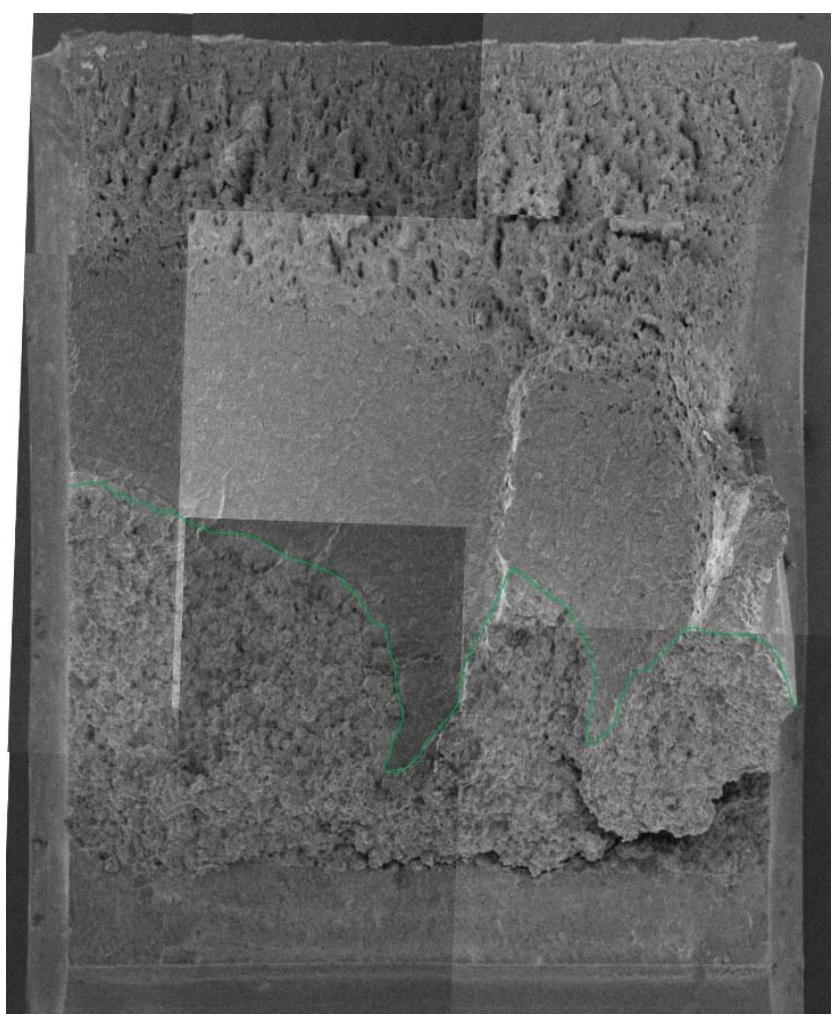

Figure 5-70: SEM micrograph showing fracture surface of specimen 10A0001B07 of XM-19 irradiated to 1.32 dpa. 


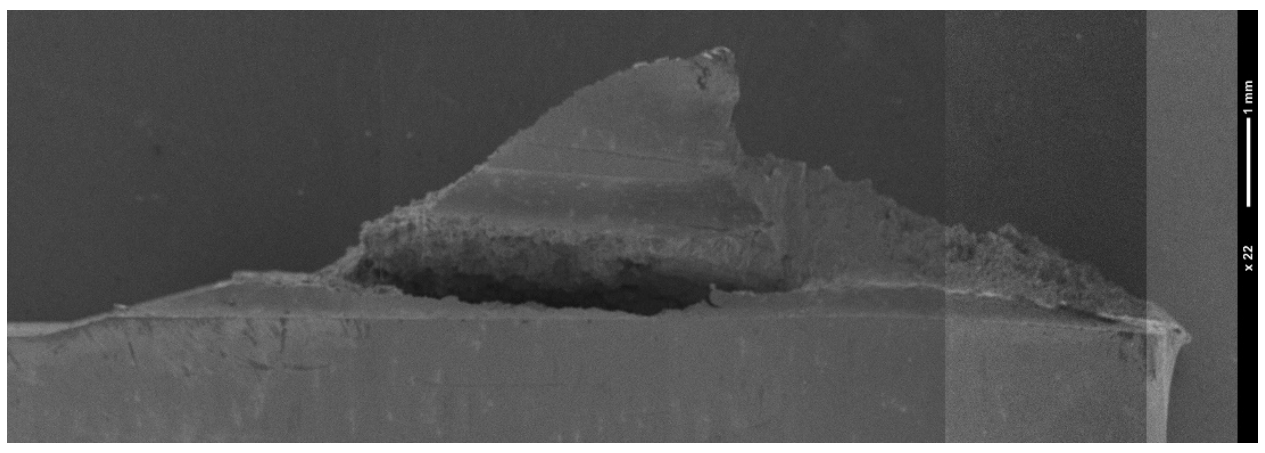

Figure 5-71: Side view of specimen 10A0001B07 of XM-19 irradiated to 1.32 dpa showing out-ofplane cracking.

\subsubsection{Specimen 10A0001D02 (1.44 dpa) XM-19 IASCC test}

Figure 5-72 provides an overview of the response of specimen 10A0001D02, which was started in $288^{\circ} \mathrm{C}$ pure water with $2.5 \mathrm{ppm} \mathrm{O}_{2}$. The in-situ fatigue pre-cracking response is shown in Figure 5-73. In each graph, the label in the outlined box represents the starting condition for that plot.

After fatigue pre-cracking, intergranular transitioning was initiated at 3023 hours at $K_{\max }=27.5$ $\mathrm{MPa} \sqrt{\mathrm{m}}$, load ratio $\mathrm{R}=0.6$, and frequencies of $0.01 \mathrm{~Hz}$ followed by $0.001 \mathrm{~Hz}$, then with a $3,500 \mathrm{~s}$ hold followed by a 9,500s hold at $\mathrm{K}_{\max }$ (Figure 5-74). At 3,176 hours, a change was made to constant $\mathrm{K}$ (no cycling) conditions. The growth rate remained essentially unchanged throughout the entire transitioning process.

A change to $95 \mathrm{ppb} \mathrm{H}_{2}$ was made at 3,254 hours (Figure 5-75), and it produced a large decrease in corrosion potential and CGR, down to a value below $10^{-9} \mathrm{~mm} / \mathrm{s}$. A change back to $2.5 \mathrm{ppm} \mathrm{O}_{2}$ at 3,466 hours increased the growth rate to close to its earlier value of $8.6 \times 10^{-7} \mathrm{~mm} / \mathrm{s}$.

The test ended at 3,539 hours. Figure 5-76 is a macro photograph of the fracture surface. The average intergranular crack depth on the fracture surface is about $30 \%$ higher than was indicated by DCPD. The crack length, CGR, and $\mathrm{K}$ data were corrected in all plots. The post-test corrected $\mathrm{K}$ values were only slightly higher than intended during testing, because the error was only moderate, as was the total crack extension. Table 5-8 is a summary of all the CGRs and related conditions under periods of constant applied $\mathrm{K}$ for this test.

Table 5-8: Constant K IASCC CGR and related conditions for specimen 10A0001D02 of XM-19 irradiated to 1.44 dpa.

\begin{tabular}{|c|c|c|c|c|c|c|c|}
\hline $\begin{array}{c}\text { Test } \\
\text { Hours }\end{array}$ & $\begin{array}{c}\mathbf{K} \\
(\mathbf{M P a V m})\end{array}$ & Chemistry & Sulfate & $\begin{array}{c}\text { Outlet } \\
\text { Cond } \\
(\boldsymbol{\mu} \mathbf{S} / \mathbf{c m})\end{array}$ & $\begin{array}{c}\text { Time } \\
\text { Increment } \\
(\mathbf{h r})\end{array}$ & $\begin{array}{c}\text { Growth } \\
\text { Increment } \\
(\mathbf{m m})\end{array}$ & $\begin{array}{c}\text { Average } \\
\text { CGR } \\
(\mathbf{m m} / \mathbf{s})\end{array}$ \\
\hline 3176 & 27.5 & NWC & 0 & 0.091 & 78 & 0.226 & $7.70 \mathrm{E}-07$ \\
\hline 3254 & 27.5 & HWC & 0 & 0.059 & 212 & 0.029 & $1.00 \mathrm{E}-09$ \\
\hline 3466 & 27.5 & NWC & 0 & 0.092 & 73 & 0.108 & $8.90 \mathrm{E}-07$ \\
\hline
\end{tabular}




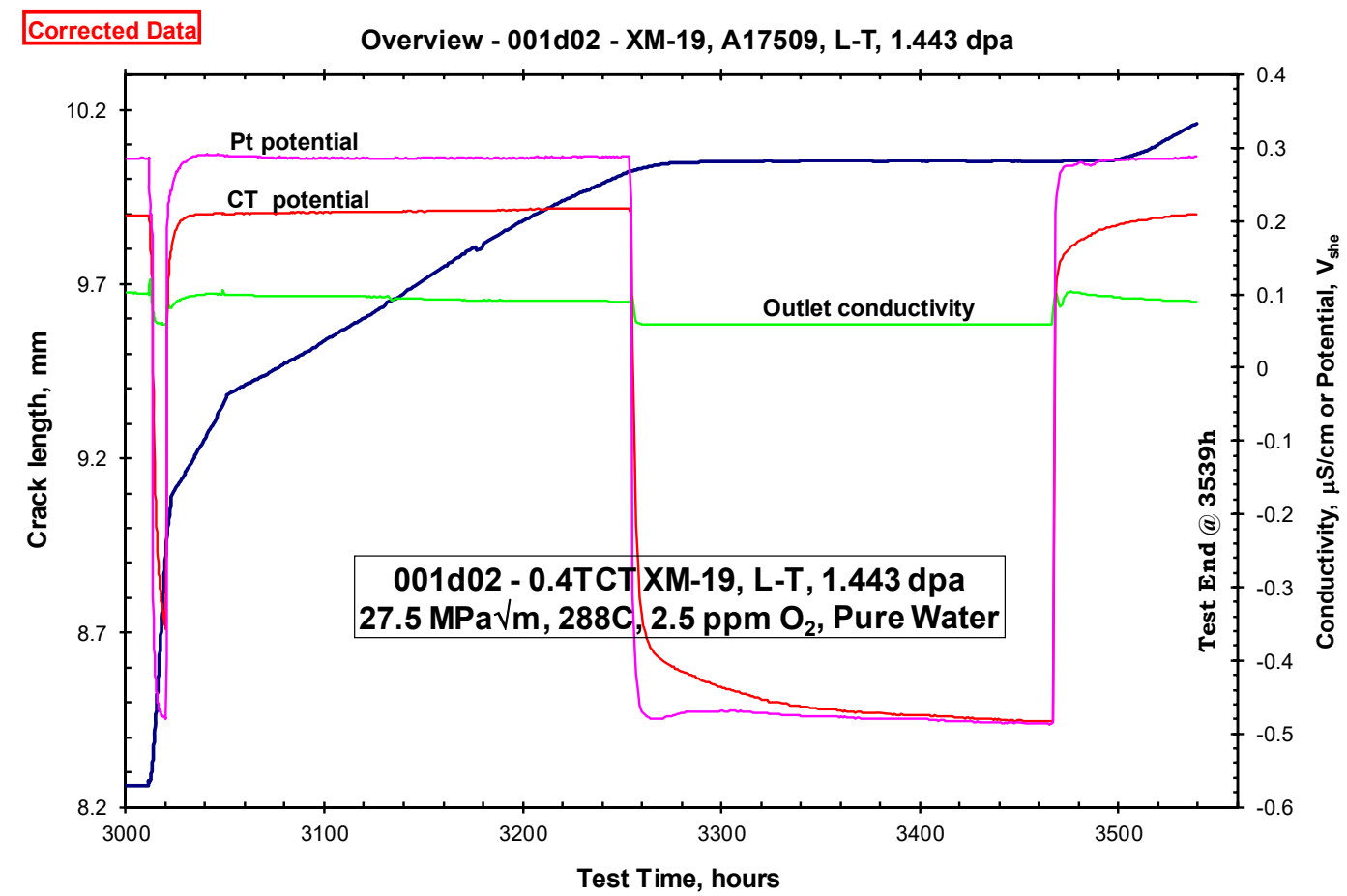

Figure 5-72: IASCC CGR test overview for specimen 10A0001D02 of XM-19 irradiated to 1.44 dpa.

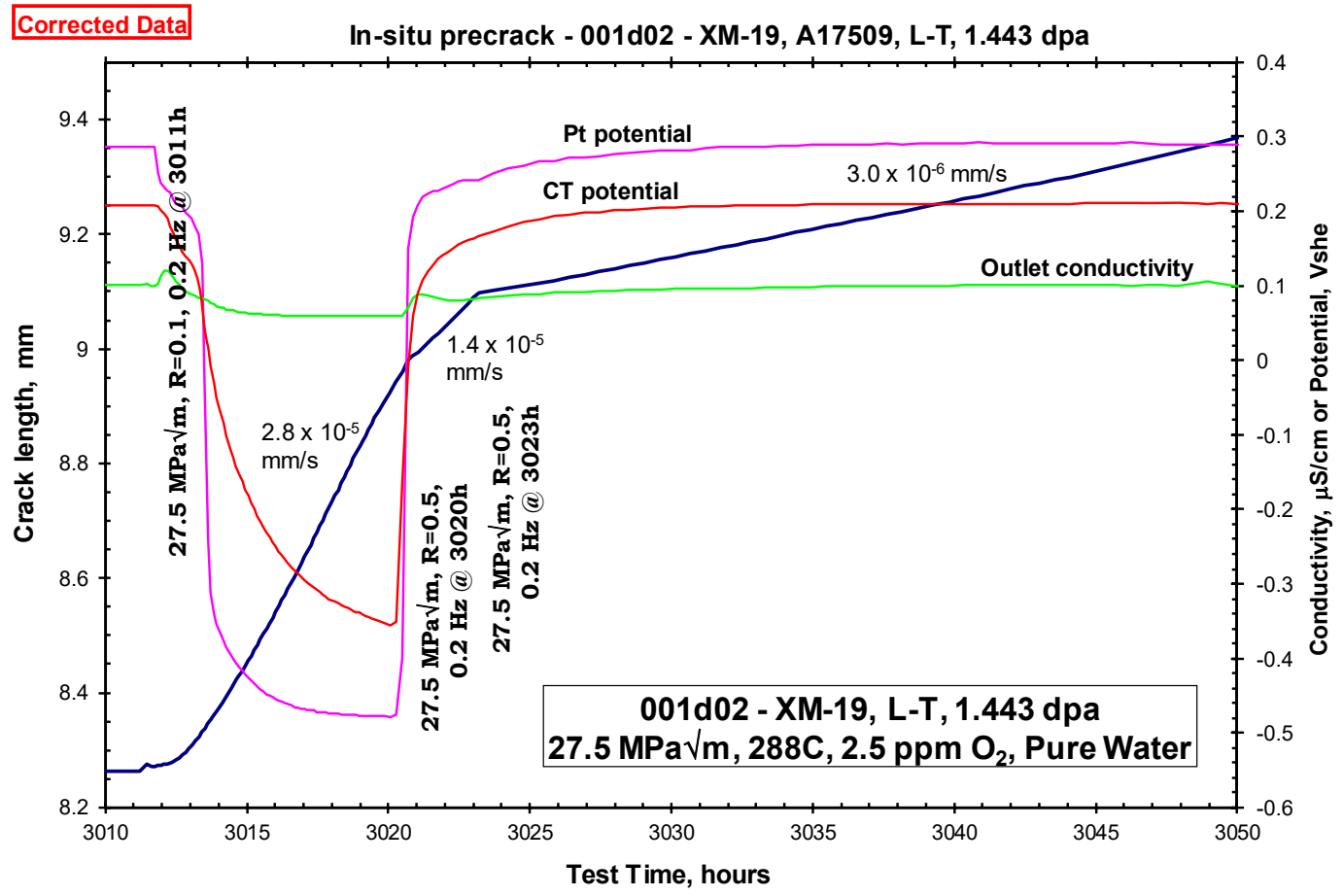

Figure 5-73: In-situ fatigue pre-crack response for specimen 10A0001D02 of XM-19 irradiated to 1.44 dpa. 


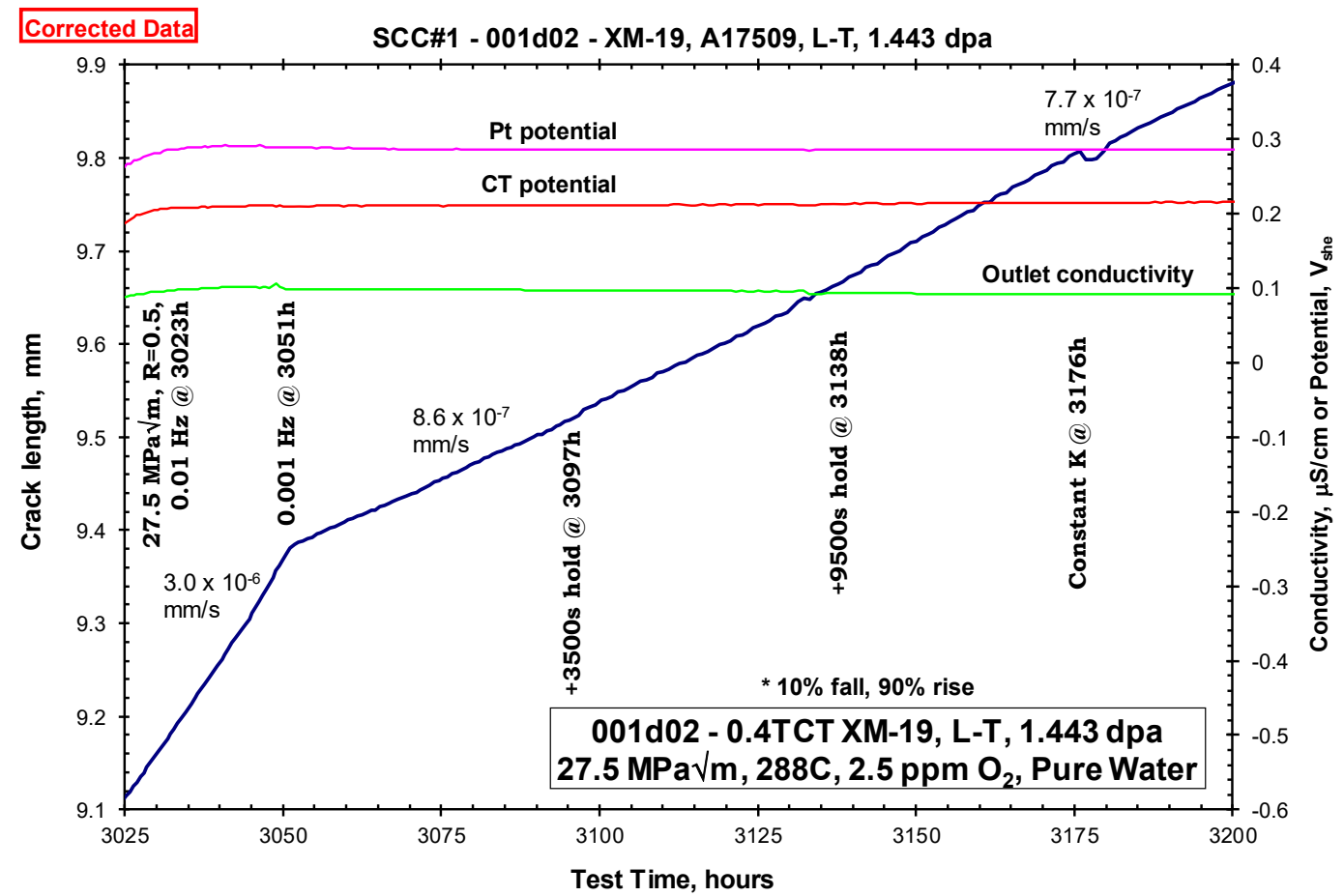

Figure 5-74: Transition to intergranular fracture for IASCC CGR test of specimen 10A0001D02 of XM-19 irradiated to 1.44 dpa.

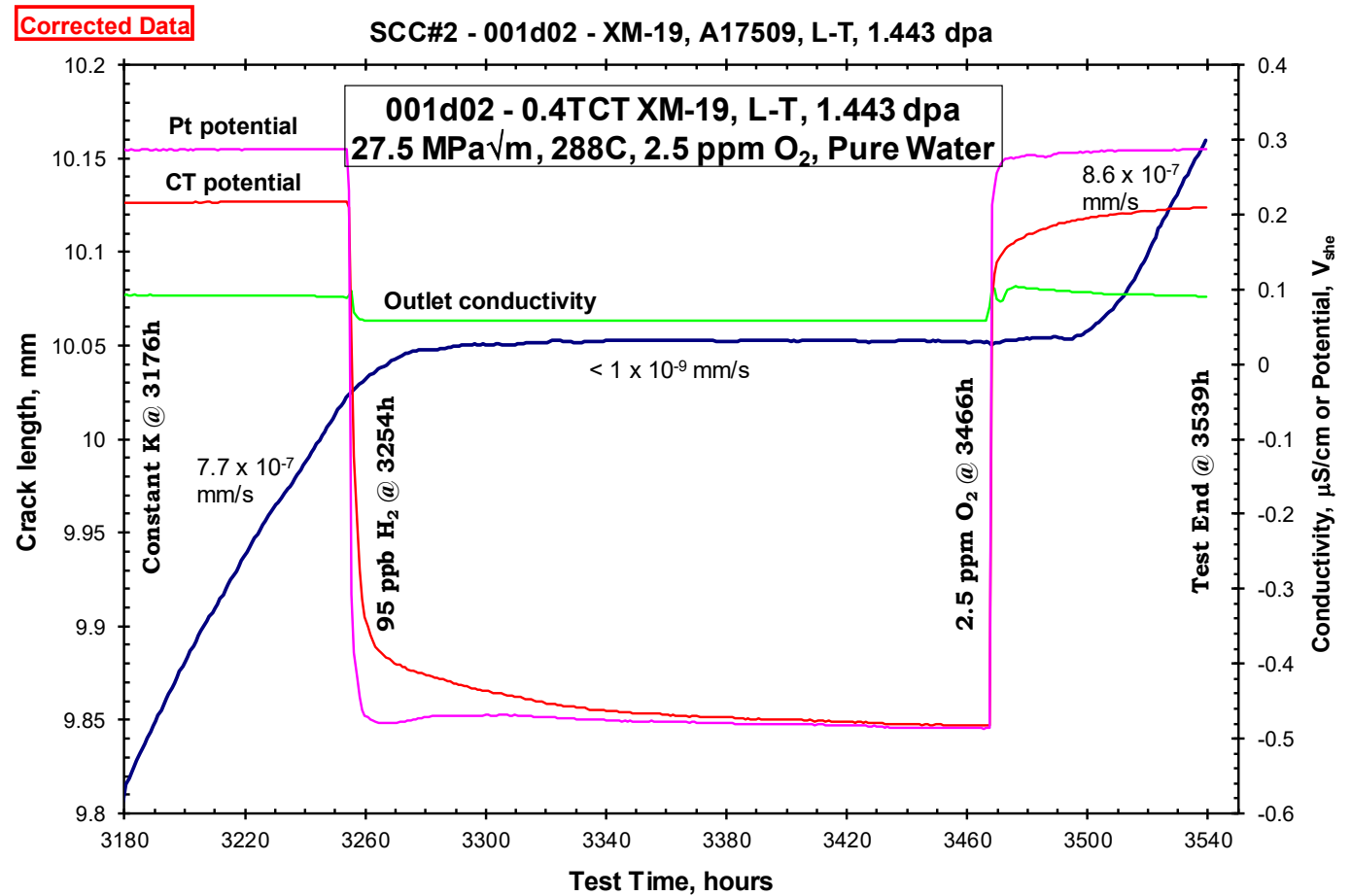

Figure 5-75: IASCC CGR test under constant $\mathrm{K}$ with transition to HWC and back to NWC for specimen 10A0001D02 of XM-19 irradiated to $1.44 \mathrm{dpa}$. 


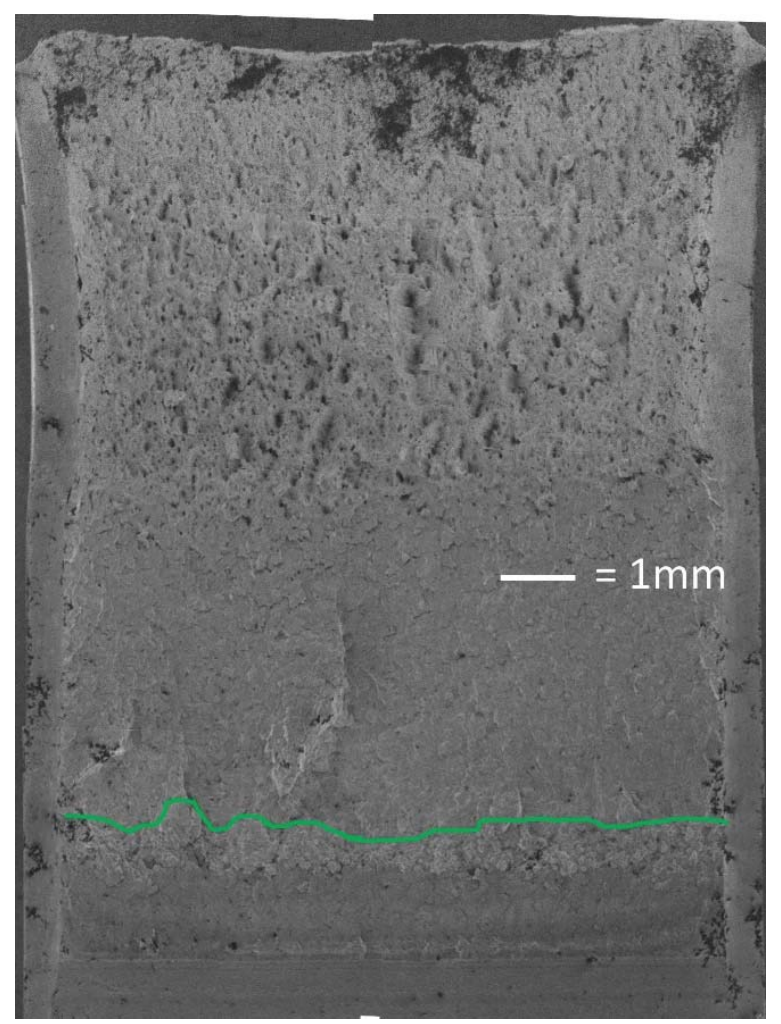

Figure 5-76: SEM micrograph showing fracture surface of specimen 10A0001D02 of XM-19 irradiated to 1.44 dpa.

\subsection{IASCC Testing Summary}

The irradiated SCC growth rate data obtained in this project are similar in quality and detailed behavior to the same heats of unirradiated alloys X-750 and XM-19 tested in other laboratories [3] [13] [14] [15] [16], as well as those observed in baseline testing conducted at INL [1]. Examples of the crack length vs. time for testing on these alloys at the other laboratories cited are shown in Figure 5-77 through Figure 5-80, which are parallel to the detailed graphs for the eight irradiated specimens tested in this project, as well as those in [1]. 


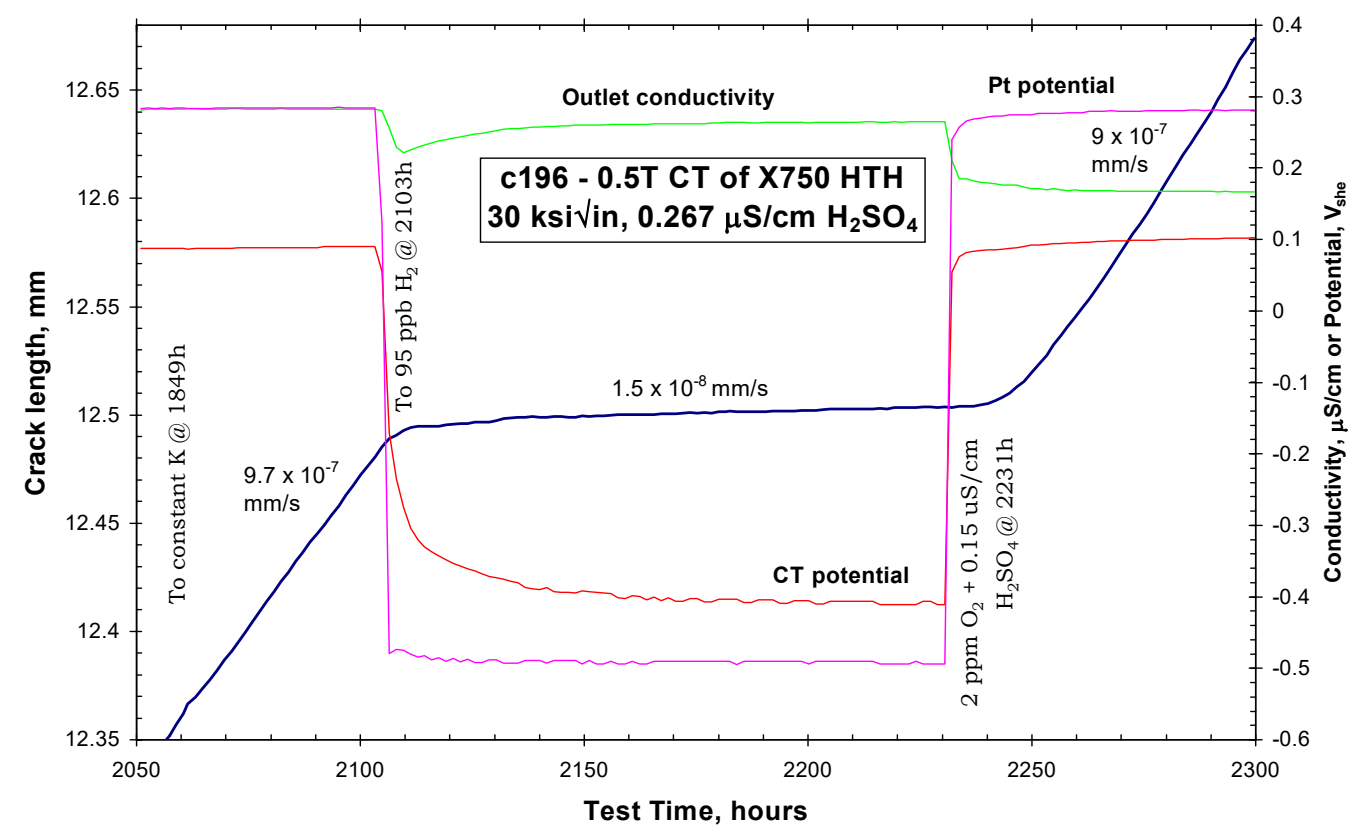

Figure 5-77: Crack length vs. time for 0.5TCT specimen of alloy X-750 in the HTH condition tested in $288^{\circ} \mathrm{C}$ water [3] [16] [15] showing very similar behavior to irradiated alloy $\mathrm{X}-750$ in this project.

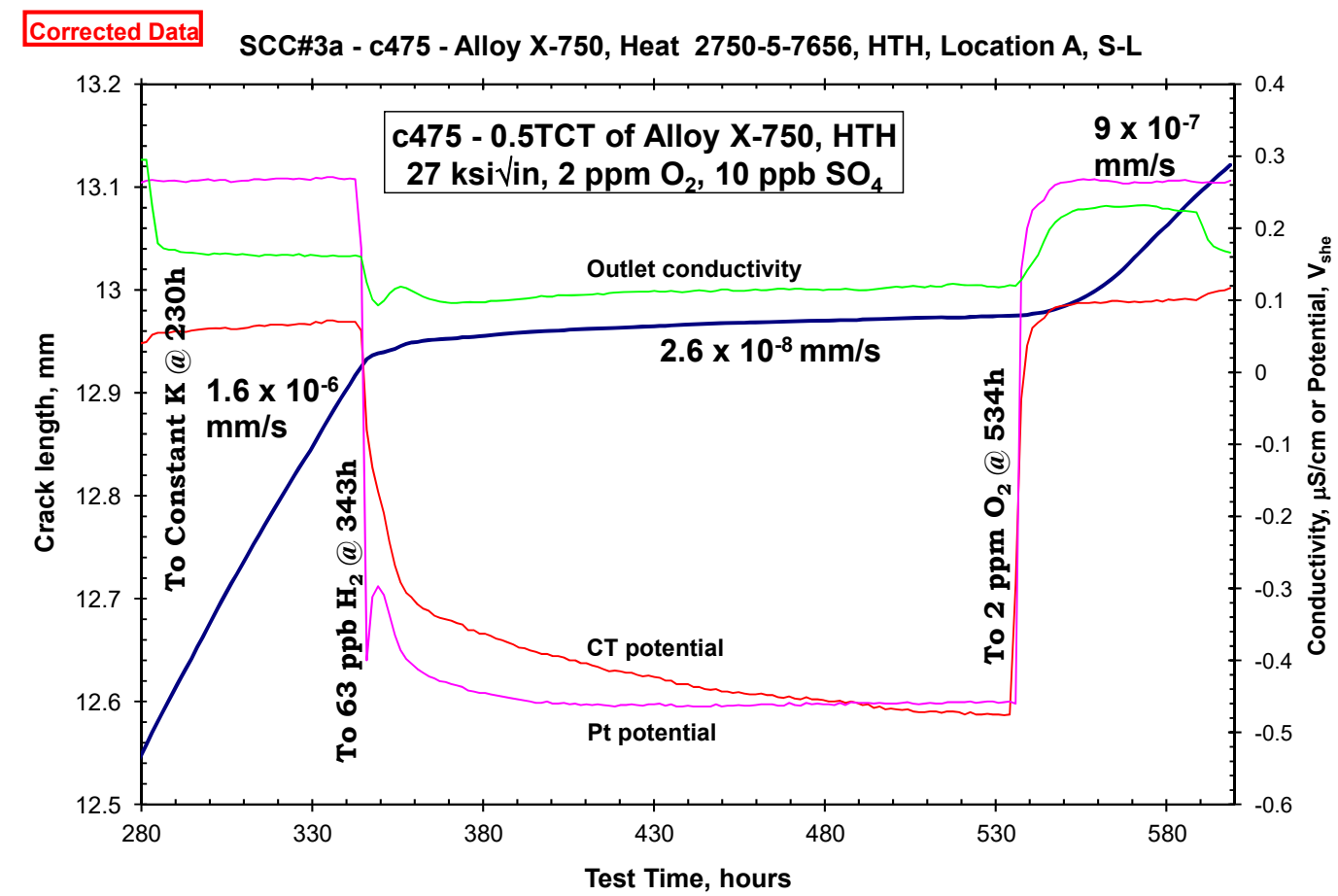

Figure 5-78: Crack length vs. time for 0.5TCT specimen of alloy X-750 in the HTH condition tested in $288^{\circ} \mathrm{C}$ water [3] [16] [15] showing very similar behavior to irradiated alloy $\mathrm{X}-750$ in this project. 


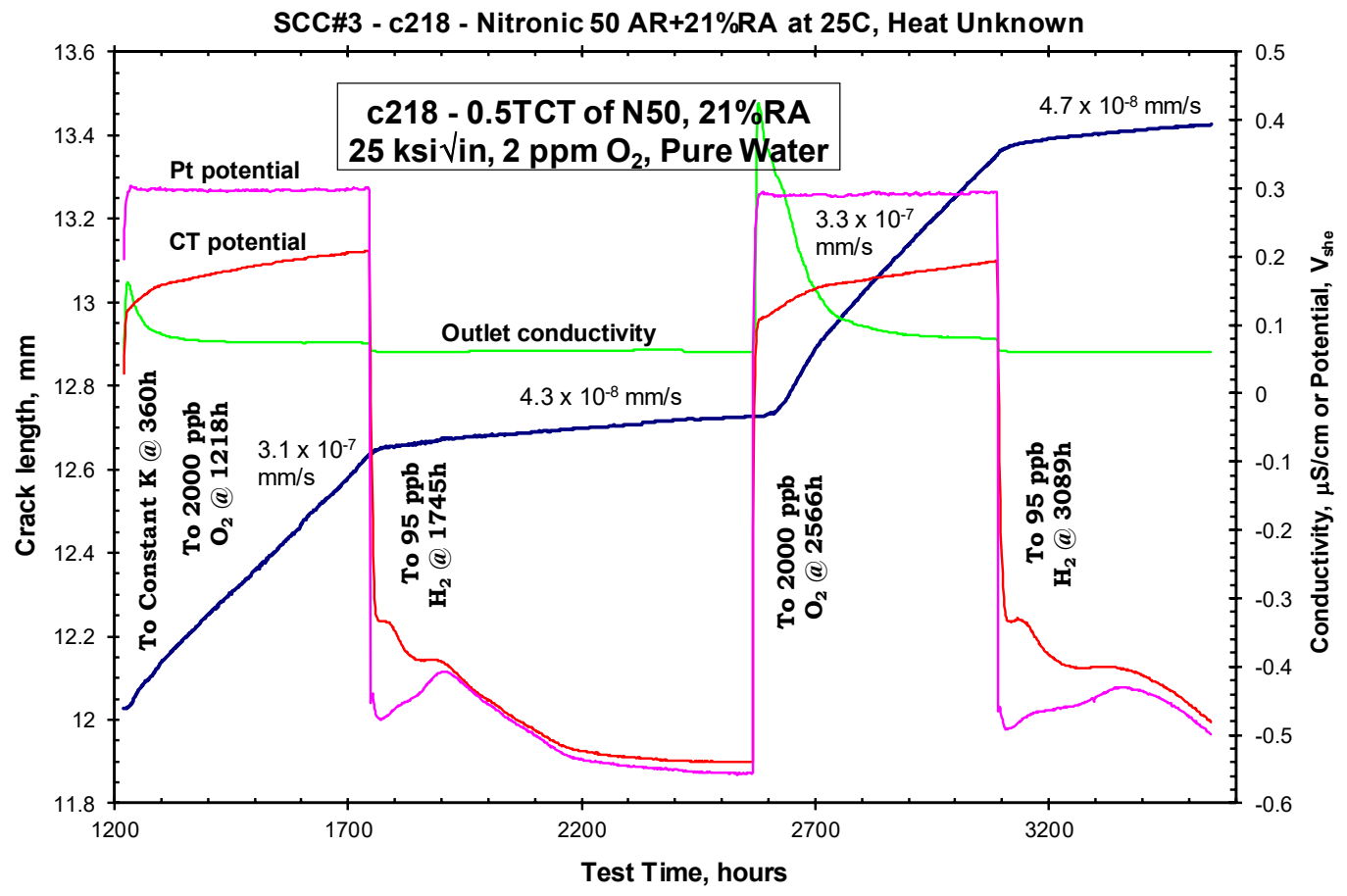

Figure 5-79: Crack length vs. time for 0.5TCT specimen of XM-19 (Nitronic 50) with $21 \%$ cold work tested in $288^{\circ} \mathrm{C}$ water [15] [14] showing similar behavior to irradiated XM-19 in this project.

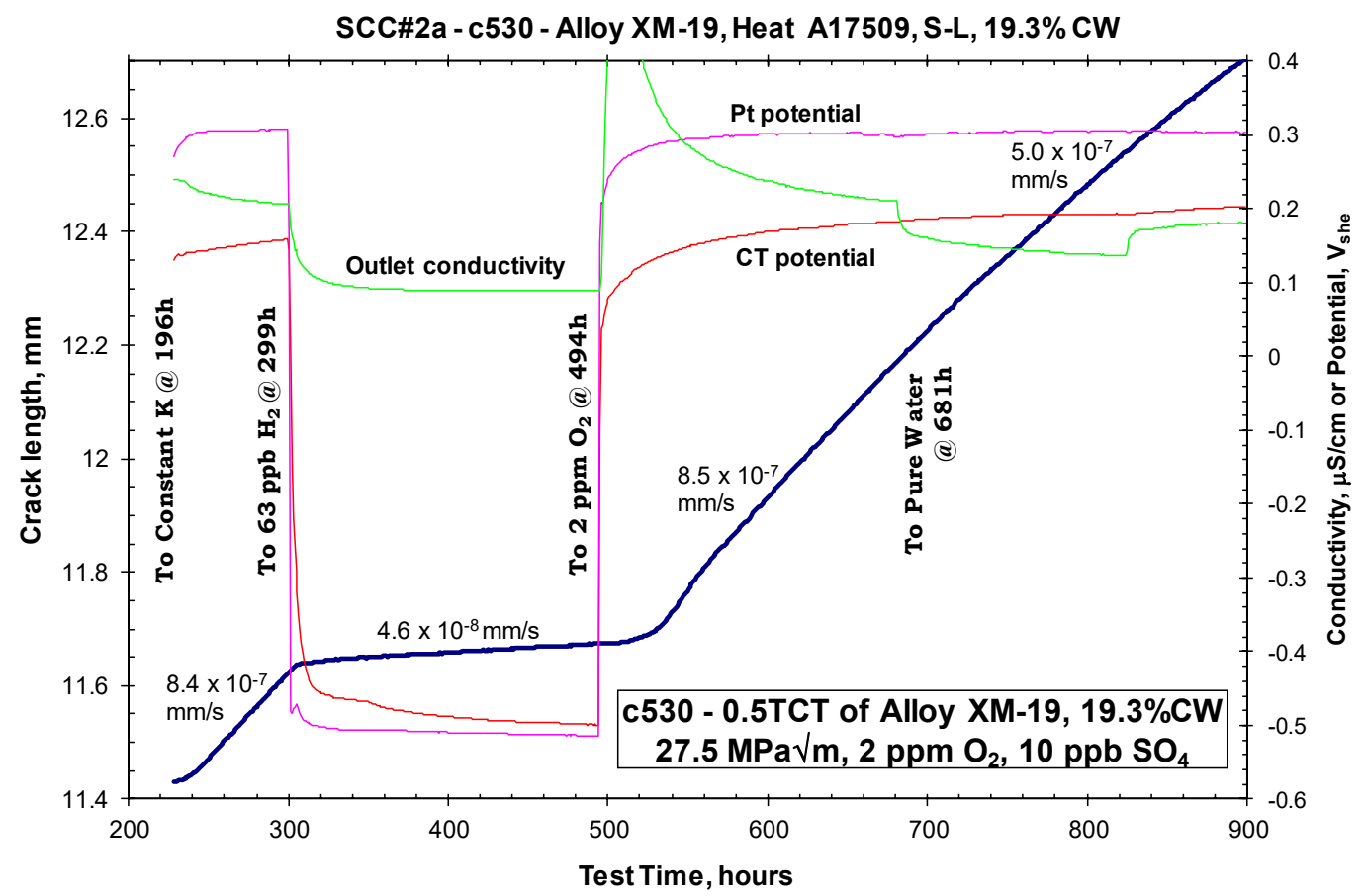

Figure 5-80: Crack length vs. time for 0.5TCT specimen of XM-19 (Nitronic 50) with 19\% cold work tested in $288^{\circ} \mathrm{C}$ water [15] [14] showing similar behavior to irradiated XM-19 in this project.

A database of the unirradiated alloy X-750 was created and evaluated [15] [14], and the SCC growth rate vs. $\mathrm{K}$ is shown in Figure 5-81, with the data separated into high and low electrochemical corrosion 
potential (ECP). The curves that are shown represent a fit to this moderately large dataset. The equivalent plots for the irradiated data are shown in Figure 5-82 and Figure 5-83, which include all fluence levels of alloys X-750 and XM-19, with the data again separated into high and low ECP. The data in Figure 5-83 are simplistically screened to reflect the higher quality data based on the transitioning behavior and SCC response (for example, growth rates that decay vs. time would be rated as lower quality). The curves shown on Figure 5-82 and Figure 5-83 are "schematic" (not fitted to the data), but in the mid-K range, they are similar to those shown in Figure 5-81.

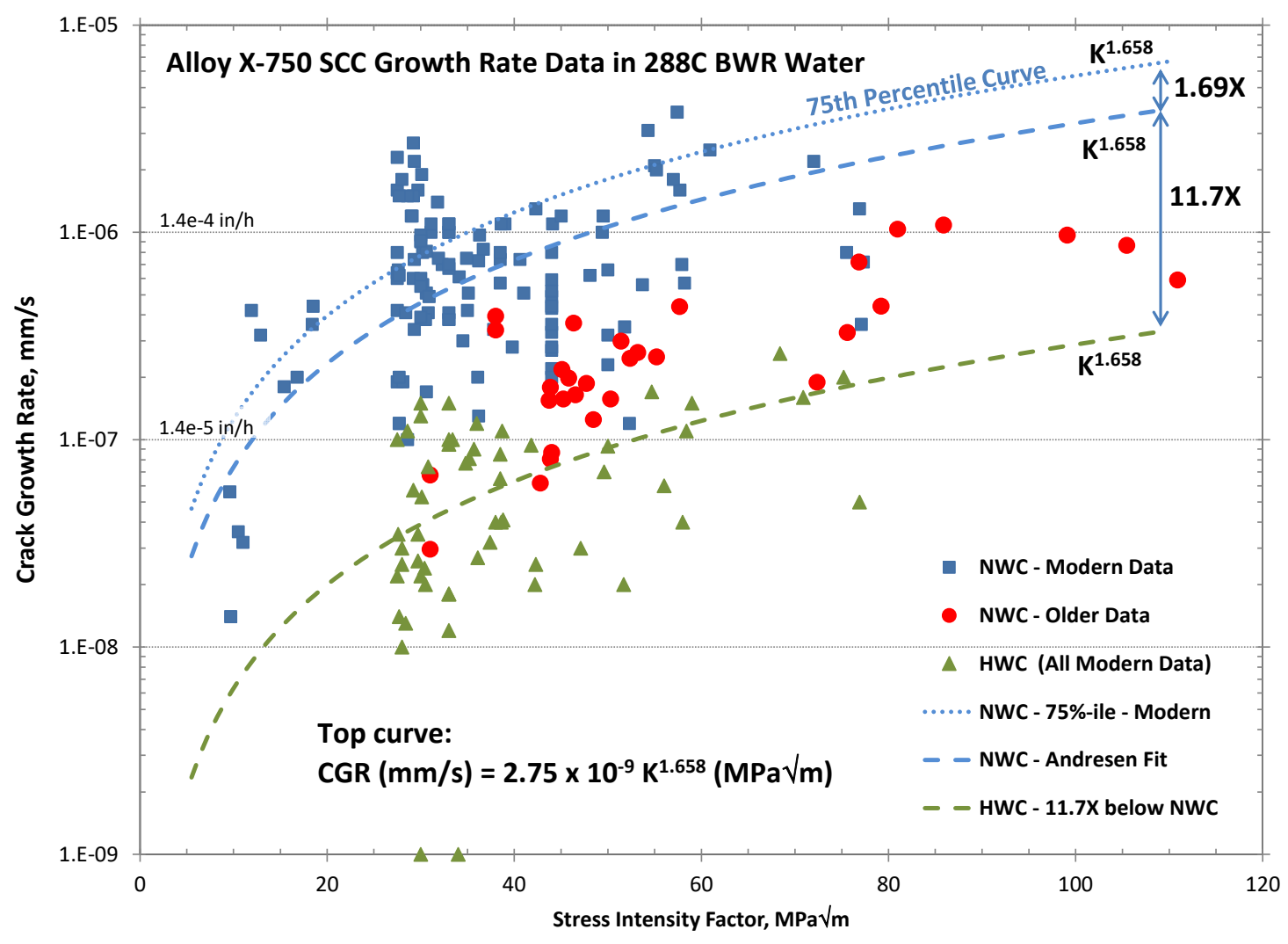

Figure 5-81: Crack growth rate vs. stress intensity factor $(K)$ for unirradiated alloy $X-750$, separated into high and low ECP data [15] [14]. 


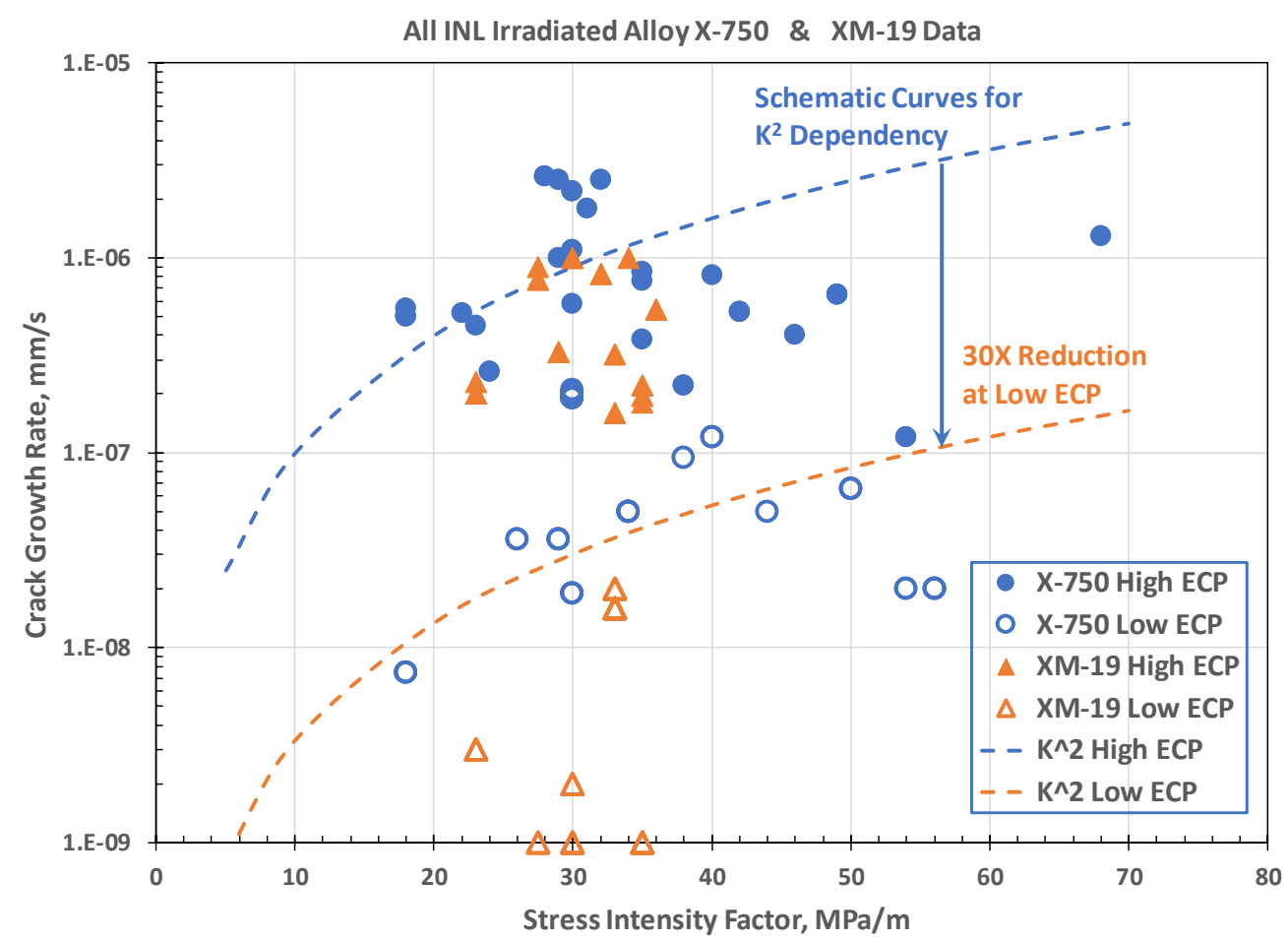

Figure 5-82: Crack growth rate vs. stress intensity factor $(K)$ for all irradiated alloys $X-750$ and XM-19, separated into high and low ECP data.

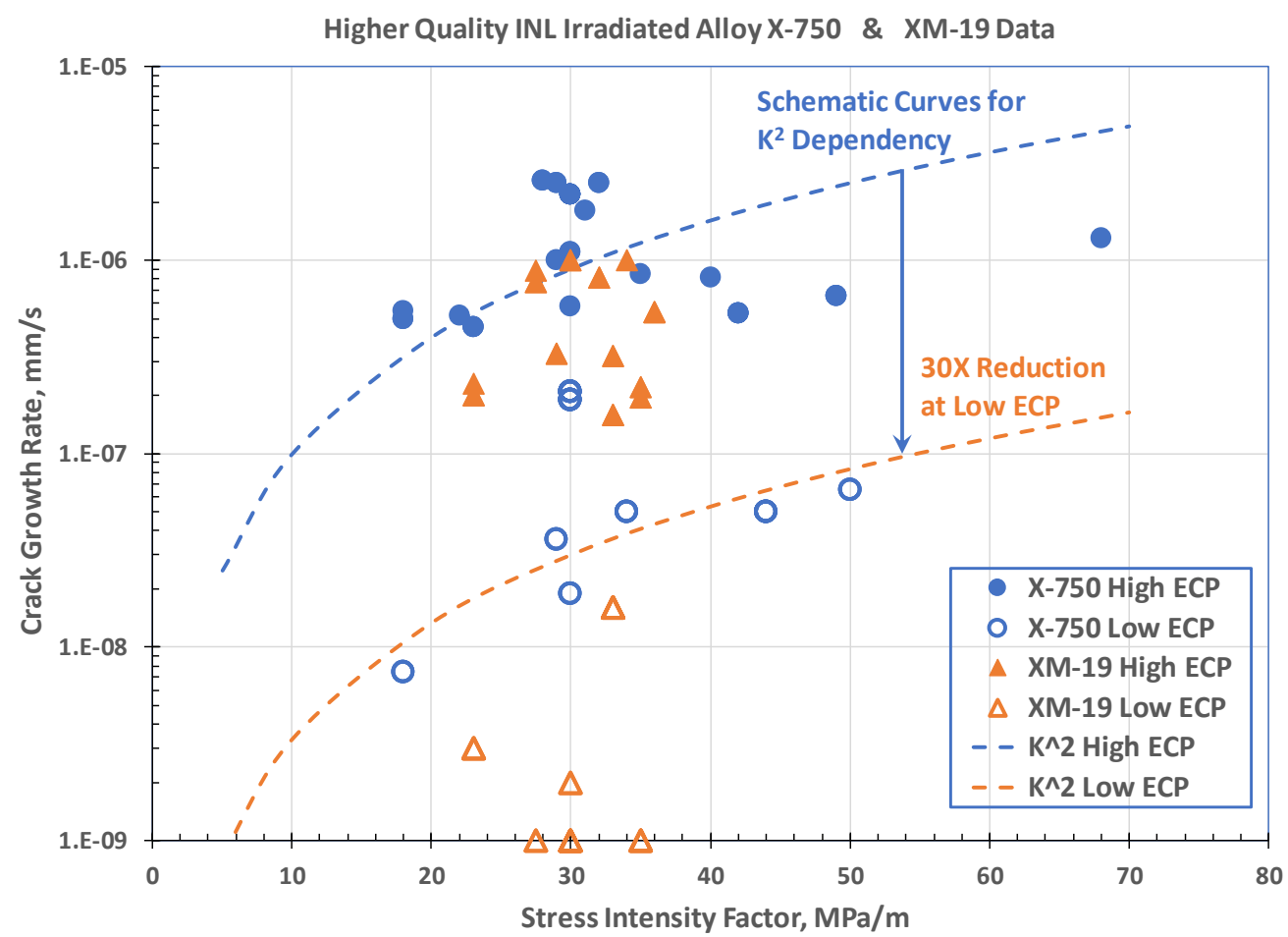

Figure 5-83: Crack growth rate vs. stress intensity factor $(K)$ for high-quality irradiated alloys $X-$ 750 and XM-19, separated into high and low ECP data. 
The benefit of HWC or low corrosion potential (ECP) on the SCC growth rate for unirradiated alloy X-750 is clear in Figure 5-81, as well as for irradiated alloys X-750 and XM-19 in Figure 5-82 and Figure 5-83. To better quantify the effect of ECP, data were identified that were obtained in adjacent on-the-fly test segments in which only the corrosion potential was changed - the exact same test conditions are present, and (essentially) the same microstructure is evaluated, but at different corrosion potential. Figure 5-84 plots these data as an HWC benefit or factor-of-improvement (the SCC growth rate at high ECP divided by the SCC growth rate at low ECP). A larger effect of ECP is observed when sulfate is present, because sulfate increases the growth rate (at these sulfate levels) only at high corrosion potential. The equivalent plots for irradiated alloys X-750 and XM-19 are shown in Figure 5-85 and Figure 5-86, and are similar to the unirradiated data. After normalizing the higher quality SCC growth rate for K using a $\mathrm{K}^{2}$ dependency, if the average SCC growth rate data at high ECP are divided by the average at low ECP, the benefit of HWC is $21.2 \mathrm{X}$ - a lower value than when evaluating adjacent on-the-fly data, which is $27.8 \mathrm{X}$ for alloy X-750, 281X for XM-19, and 126X for all on-the-fly data. This is consistent with the common observation that trends evaluated after accumulating divergent data exhibit a lower dependency than trends determined from on-the-fly or as-identical-as-possible data. Table 5-9 provides a summary of the benefits of HWC using various analysis approaches.

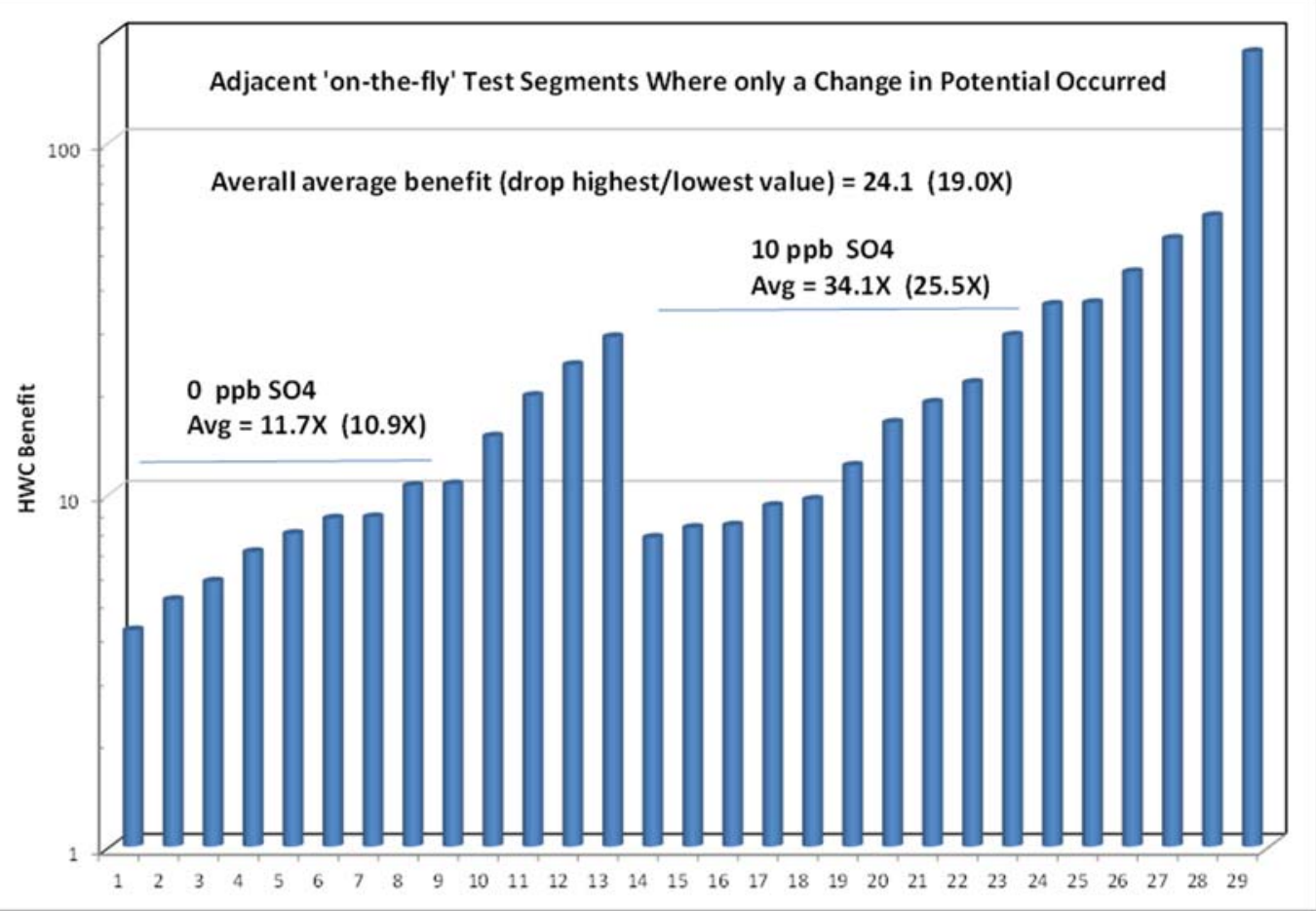

Figure 5-84: Crack growth rate benefit of shifting from high to low ECP of unirradiated alloy X750, separated into 0 and $10 \mathrm{ppb}$ sulfate data [15] [14]. 


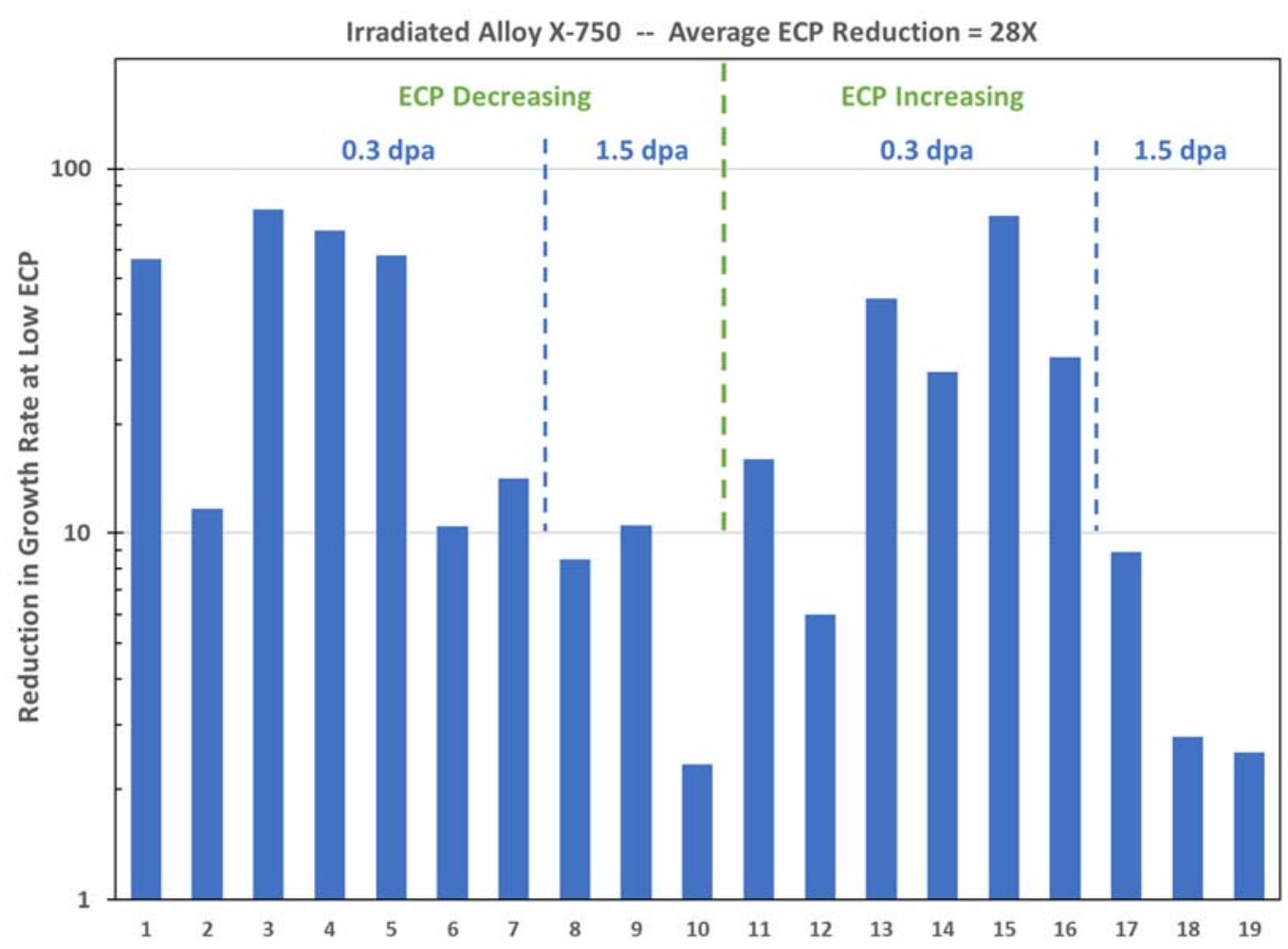

Figure 5-85: Crack growth rate benefit of shifting from high to low ECP of irradiated alloy X-750 in this project.

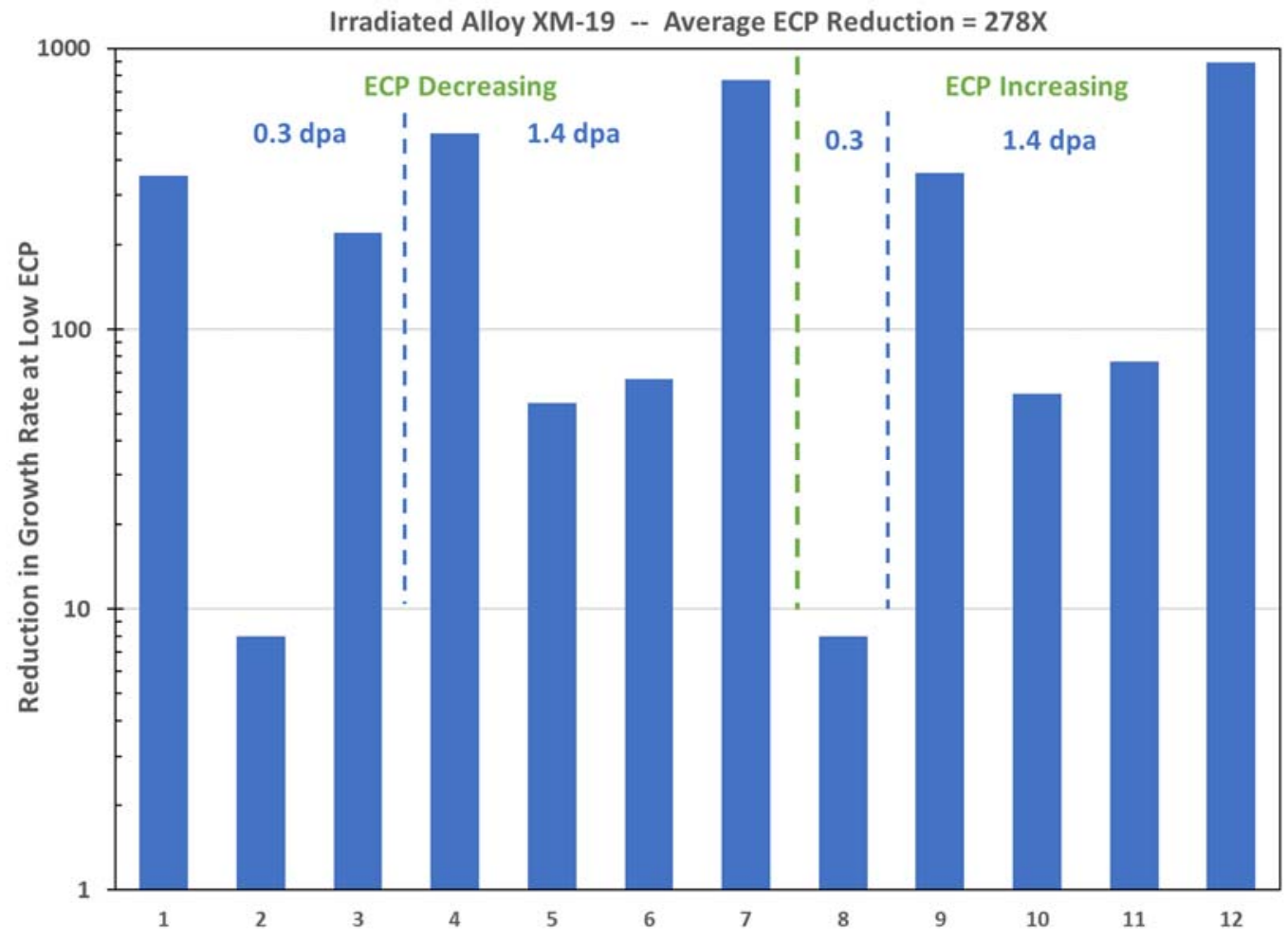

Figure 5-86: Crack growth rate benefit of shifting from high to low ECP of irradiated XM-19 in this project. 
Table 5-9: Growth rate benefit of HWC evaluated using different subsets of data-calculated as [growth rate at high ECP] divided by [growth rate at low ECP].

\begin{tabular}{|c|c|c|}
\hline & Falling ECP & Rising ECP \\
\hline Average of All Rising \& Falling ECP Data & \multicolumn{2}{|c|}{$126 \mathrm{X}$} \\
\hline & \multicolumn{2}{|c|}{$28 \mathrm{X}$} \\
\hline Average of Rising \& Falling Alloy X-750 ECP Data & $32 X$ & $24 X$ \\
\hline Average of All Fluence X-750 Data & $47 X$ & $33 X$ \\
\hline Average of Alloy X-750 Low Dose Data & $\mathbf{8 . 8 X}$ & $4.7 X$ \\
\hline Average of Alloy X-750 High Dose Data & & $281 X$ \\
\hline Average of All Rising \& Falling XM-19 ECP Data & & $279 X$ \\
\hline Average of All Fluence XM-19 Data & $282 X$ & $\mathbf{8 . 0 X}$ \\
\hline Average XM-19 Low Dose Data & $194 X$ & $347 X$ \\
\hline Average XM-19 High Dose Data & $348 X$ & \\
\hline
\end{tabular}

On-the-fly effects of sulfate on the SCC growth rate of unirradiated alloy X-750 are shown in Figure 5-87, and these are similar to the data obtained in this project on irradiated alloy X-750 (Figure 5-88). In all cases, the effect of $10-30 \mathrm{ppb}$ sulfate in these materials is limited, averaging about $1.5 \mathrm{X}$ higher growth rates with sulfate present, and only at high ECP.

Like other parameters that cannot be evaluated in on-the-fly experiments, the effect of stress intensity factor $(\mathrm{K})$ tends to be masked or diluted when data obtained at different times, in different laboratories, and/or in different tests are accumulated and evaluated. Figure 5-89 shows that this can be mitigated to some extent by screening the unirradiated alloy X-750 data, but the $\mathrm{K}$ dependency in the database (Figure $5-89$, lower) is somewhat lower than the $\mathrm{K}$ dependency in single specimen tests in which $\mathrm{K}$ is progressively evaluated (Figure 5-89, upper). Figure 5-90 shows the effect of the stress intensity factor on the SCC growth rate of individual specimens of unirradiated XM-19, which is broadly consistent with a $\mathrm{K}^{2}$ dependency.

The effect of neutron fluence is shown in Figure 5-91 and Figure 5-92, with data separated into high and low ECP. At high ECP (Figure 5-91), there is only limited evidence that the higher fluence specimens ( $\sim 1.5 \mathrm{dpa})$ exhibit a higher SCC growth rate than do the lower fluence specimens $(\sim 0.3 \mathrm{dpa})$. The effect at low ECP (Figure 5-92) indicates a large effect of fluence, with the $\sim 1.5$ dpa data in almost all cases showing higher CGR than the $\sim 0.3$ dpa data. 


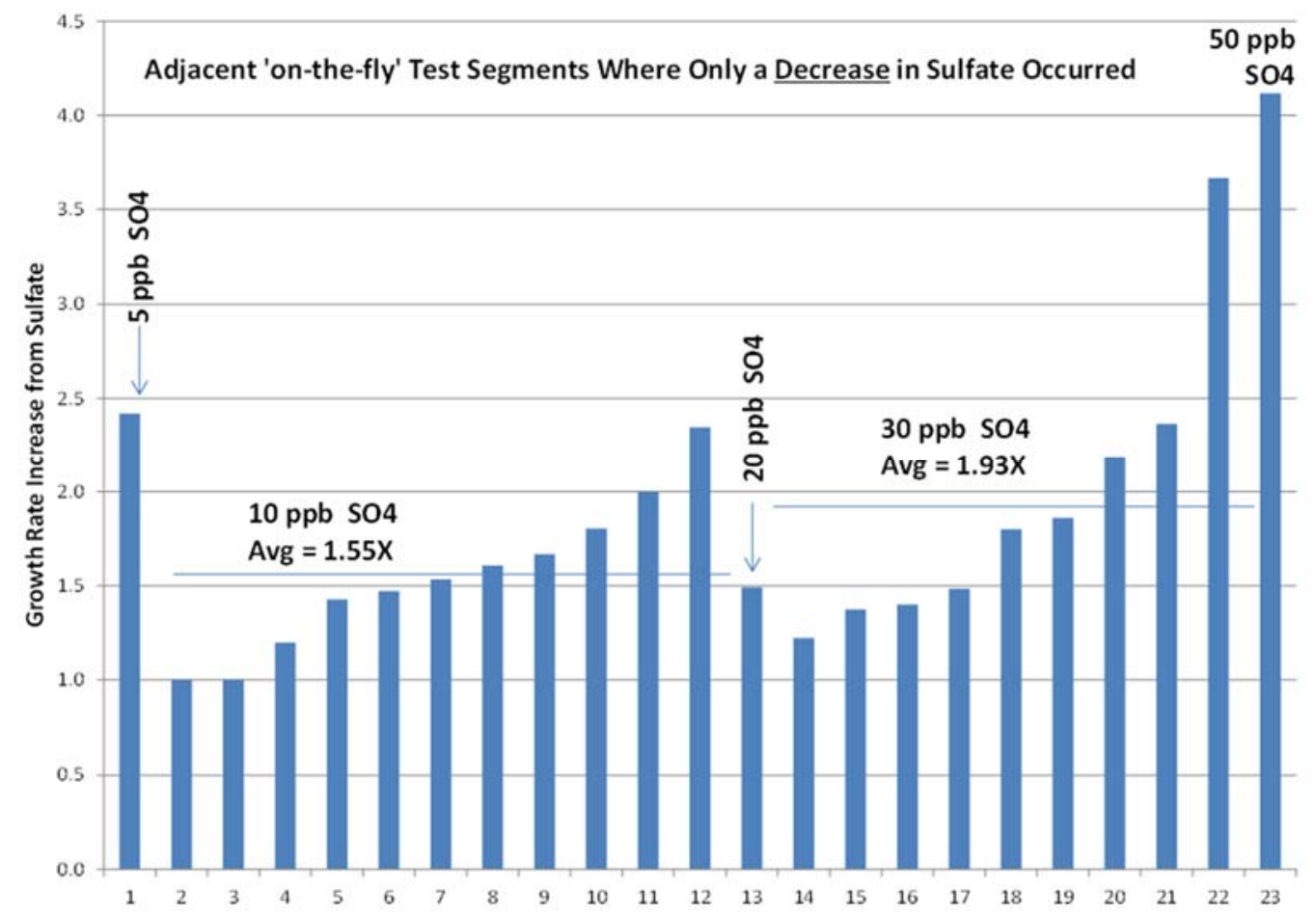

Figure 5-87: Crack growth rate benefit of shifting from pure water to 10 or $30 \mathrm{ppb}$ sulfate in unirradiated alloy $X-750$ [15] [14].

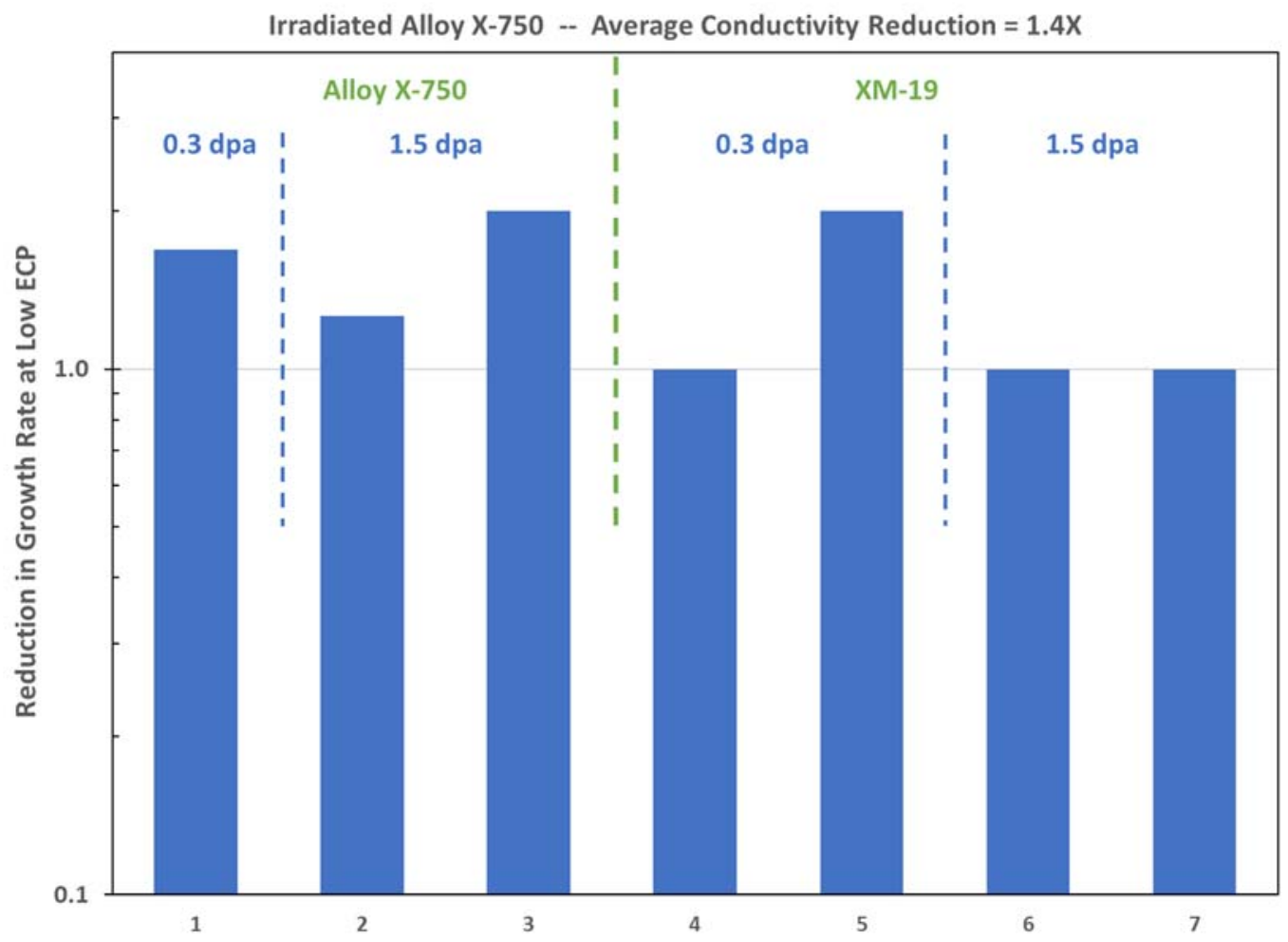

Figure 5-88: Crack growth rate benefit of shifting from pure water to $10 \mathrm{or} 30 \mathrm{ppb}$ sulfate in irradiated alloy $\mathrm{X}-750$ in this project. 

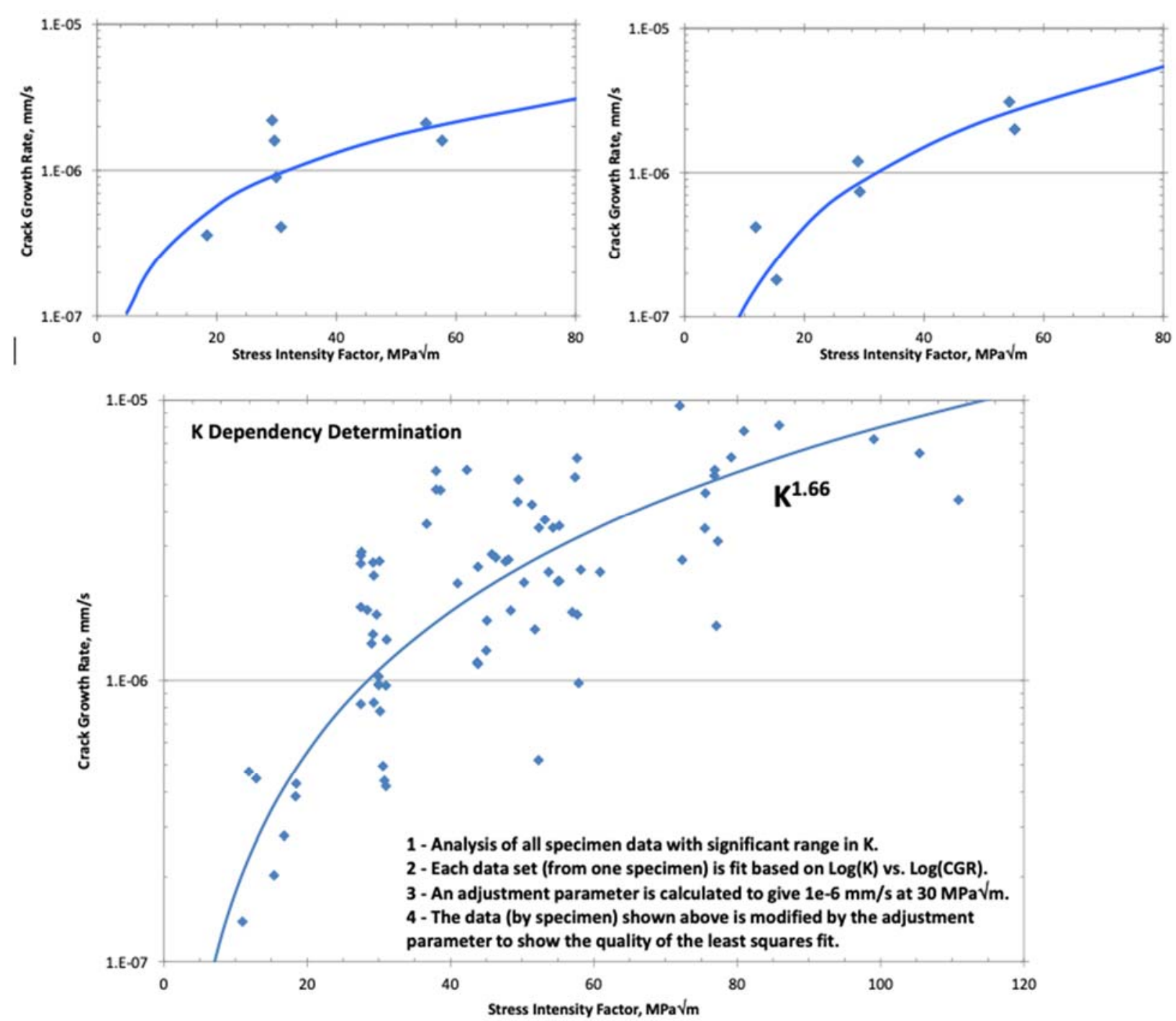

Figure 5-89: The dependency of stress intensity factor (K) on SCC growth rate in individual specimens (upper plots), and a screened database (lower plot) of unirradiated alloy X-750 [15] [14]. 


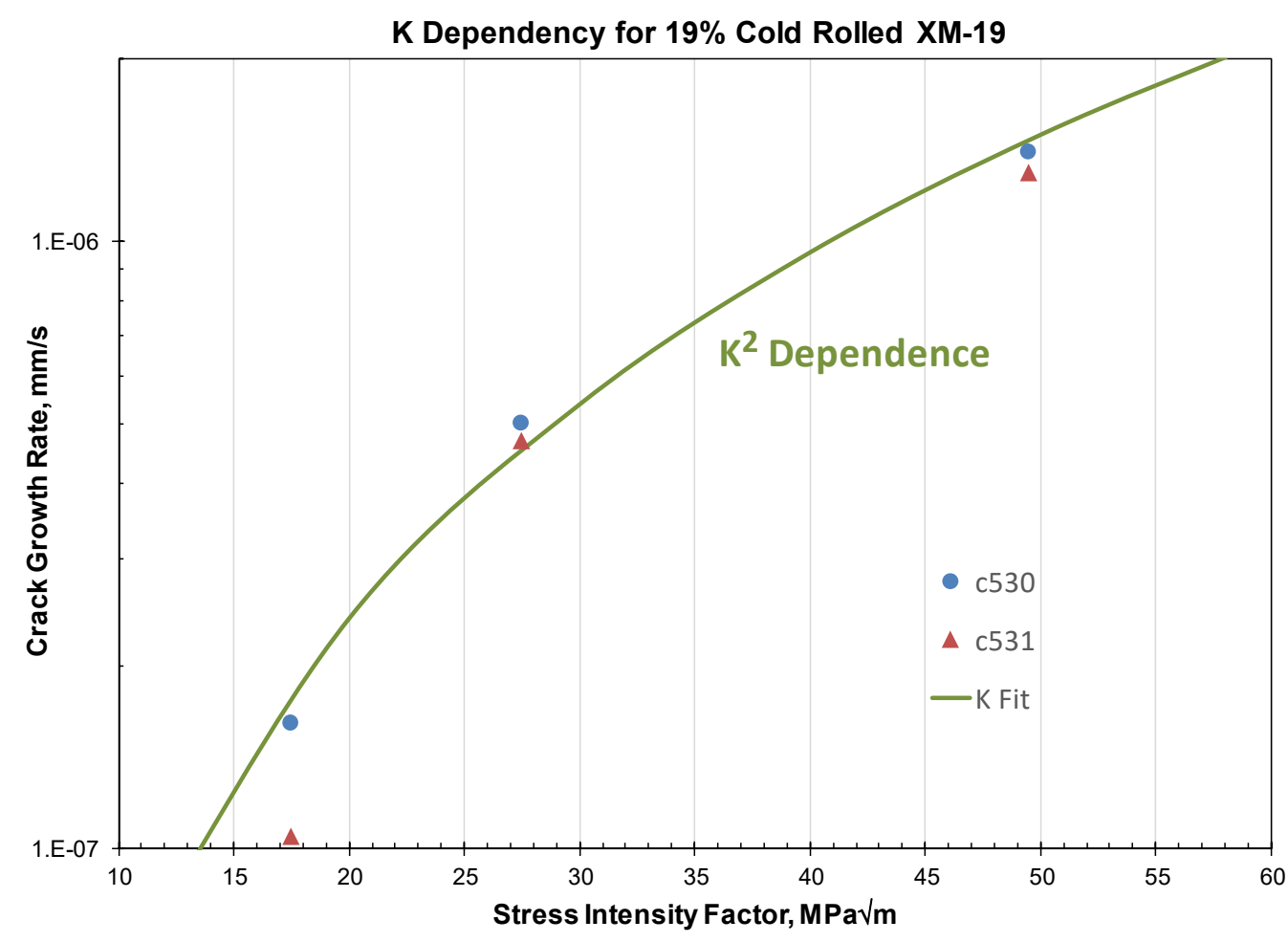

Figure 5-90: The dependency of stress intensity factor (K) on SCC growth rate in individual specimens of unirradiated XM-19 [15] [14].

Higher Quality, High ECP INL Irradiated Alloy X-750 \& XM-19 Data

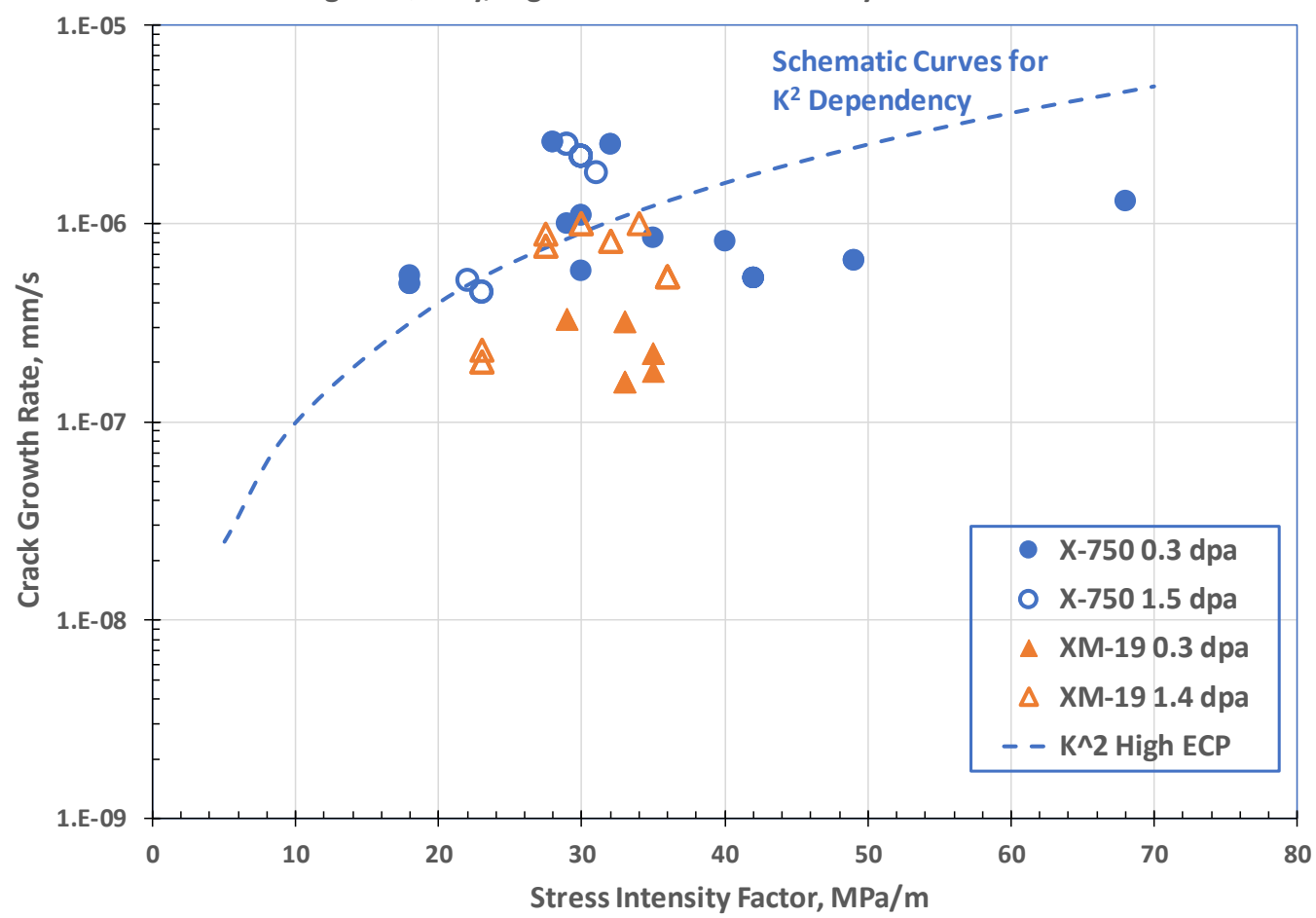

Figure 5-91: SCC growth rate vs. stress intensity factor (K) for irradiated alloys X-750 and XM-19, showing the effect of higher vs. lower fluence at high ECP in this project. 


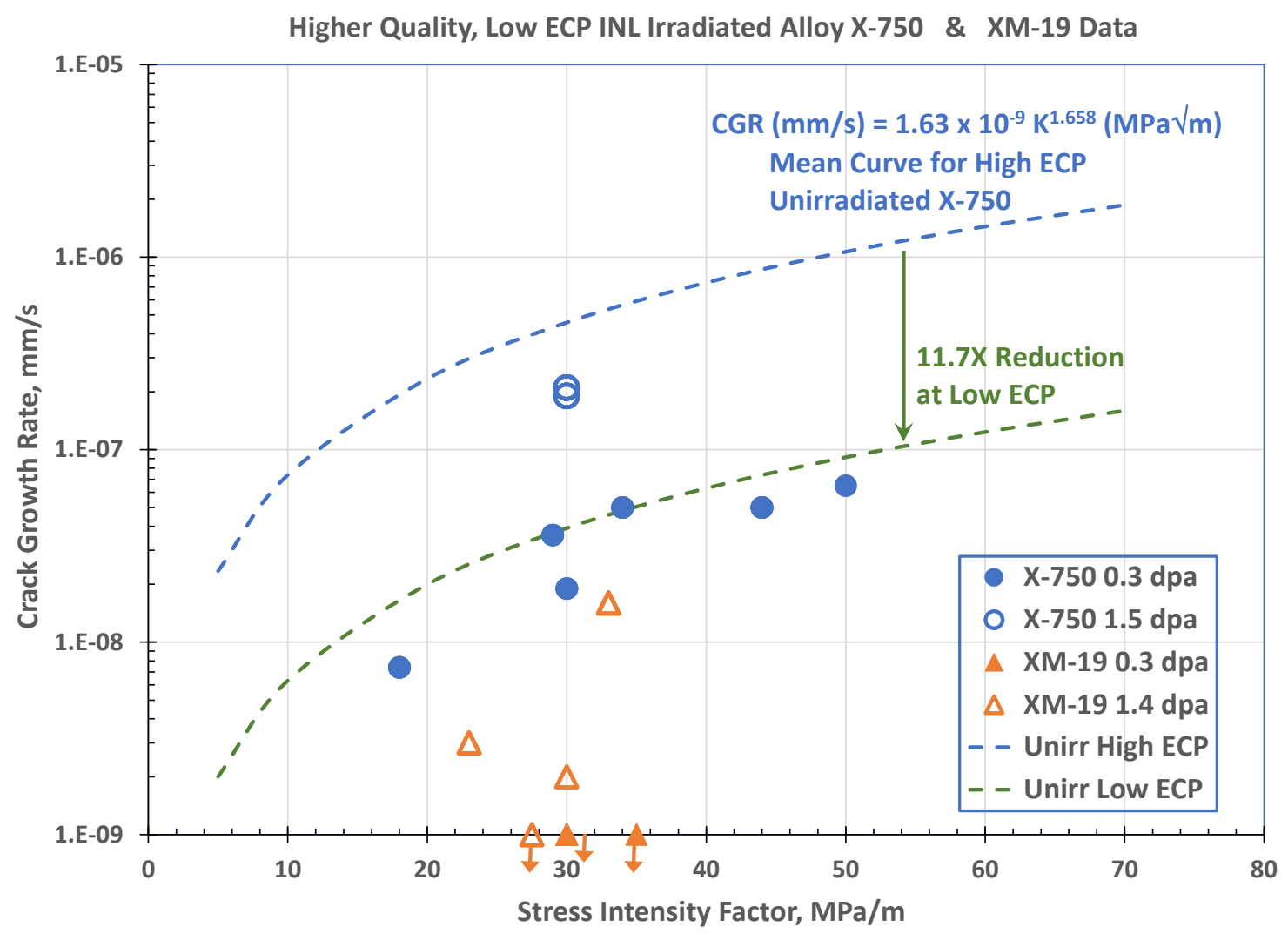

Figure 5-92: SCC growth rate vs. stress intensity factor (K) for alloys X-750 and XM-19, showing the effect of higher vs. lower fluence at low ECP.

\section{Transmission Electron Microscopy}

For microstructural characterization, transmission electron microscopy (TEM) lamellae were prepared using a shielded FEI Quanta 3D FEG DualBeam focused ion beam/scanning electron microscope (FIB/SEM). TEM lamellae were lifted out and thinned to $\sim 100 \mathrm{~nm}$ in thickness using a 30 $\mathrm{keV} \mathrm{Ga}$ ion beam, followed by cleaning with a $5 \mathrm{keV}$ and $2 \mathrm{keV} \mathrm{Ga}$ ion beam. To avoid FIB Ga ion damage, several specimens were also prepared using twin-jet polishing. The TEM and scanning transmission electron microscopy with energy dispersive x-ray spectroscopy (STEM-EDS) characterization was carried out using a FEI Themis Titan 200 FEG STEM equipped with a super-X EDS system and operated at $200 \mathrm{kV}$. A JEOL JEM-2010 LaB 6 TEM operated at $200 \mathrm{kV}$ was also used for defect analysis.

\subsection{General Unirradiated Alloy X-750 and XM-19 Characteristics}

A small amount of baseline characterization work was performed in order to characterize grain boundaries in unirradiated alloys X-750 and XM-19. STEM-EDS images of a high-angle grain boundary (HAGB) of unirradiated alloy X-750 clearly show a significant number of Cr-rich carbides, along with a low density of coarse ( $\mathrm{Ti}, \mathrm{Nb}, \mathrm{V}$ )-rich carbide precipitates (Figure 6-1). A higher magnification (Figure 6-2) STEM-EDS image shows that both $\mathrm{Cr}$-rich carbides and ( $\mathrm{Ti}, \mathrm{Al})$-rich precipitates appear on the HAGB. The Cr-rich carbides are likely $\mathrm{M}_{23} \mathrm{C}_{6}$, and the (Ti, $\left.\mathrm{Al}\right)$-rich precipitates are likely $\mathrm{Ni}_{3}(\mathrm{Ti}, \mathrm{Al}) \gamma^{\prime}$ precipitates. In order to confirm that the ( $\mathrm{Ti}-\mathrm{Al})$-rich precipitates were $\mathrm{Ni}_{3}(\mathrm{Ti}, \mathrm{Al}) \gamma^{\prime}$ precipitates, TEM diffraction patterns were taken to look for possible superlattice reflections. Figure 6-3 shows the TEM diffraction pattern of unirradiated alloy X-750 taken along the [011] zone axis, showing obvious $\gamma^{\prime}$ 
superlattice reflection spots, which confirms that the $(\mathrm{Ti}, \mathrm{Al})$-rich precipitates are $\mathrm{L}_{2}$ ordered $\gamma^{\prime}$ precipitates.

Figure 6-4 is a STEM-EDS image of a grain boundary in unirradiated XM-19. It shows a low density of coarse ( $\sim 500 \mathrm{~nm}$ diameter) $(\mathrm{Nb}, \mathrm{Mo})$-rich nitride precipitates and no noticeable segregation along the grain boundaries.
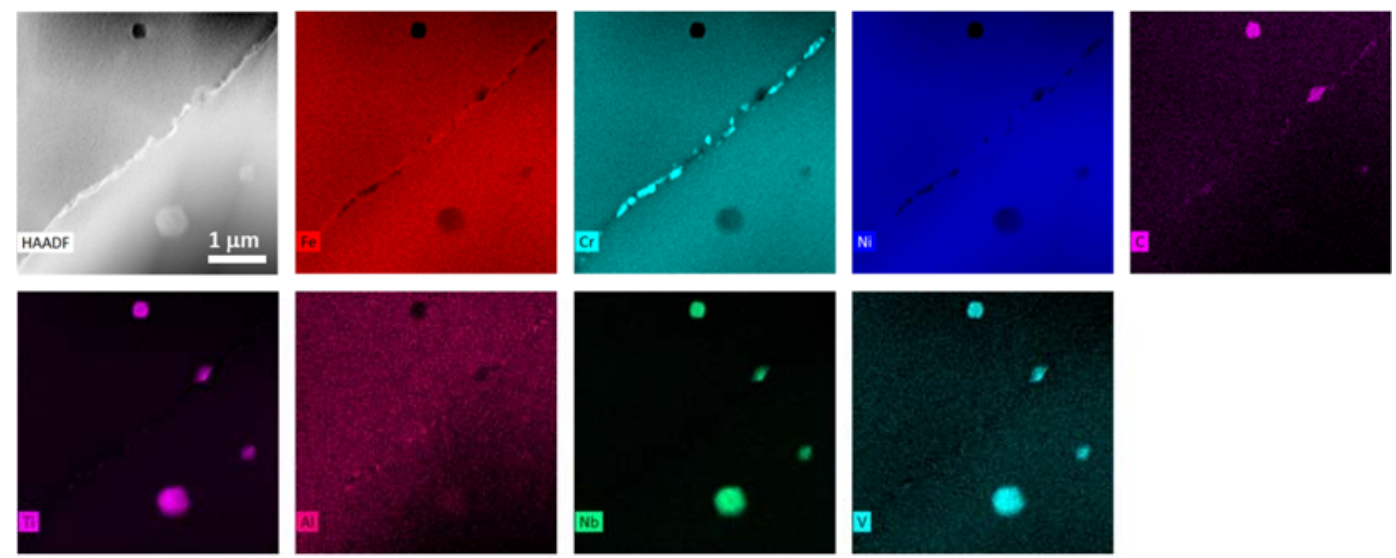

Figure 6-1: STEM-EDS image of HAGB in unirradiated alloy X-750.
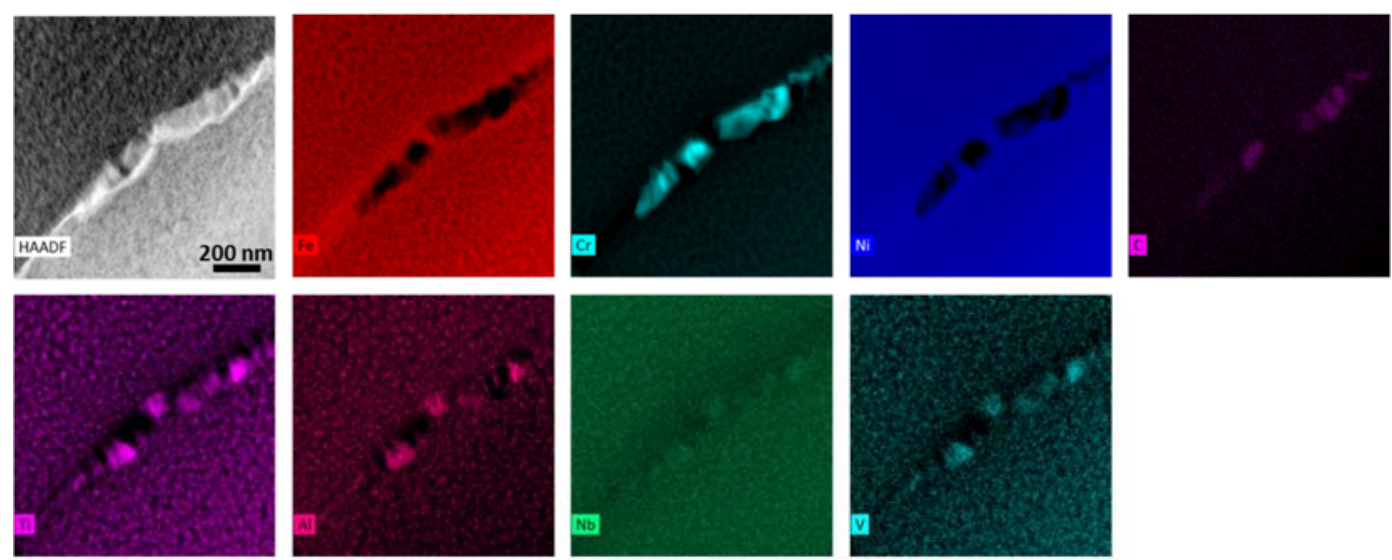

Figure 6-2: High magnification STEM-EDS image of HAGB in unirradiated alloy X-750. 

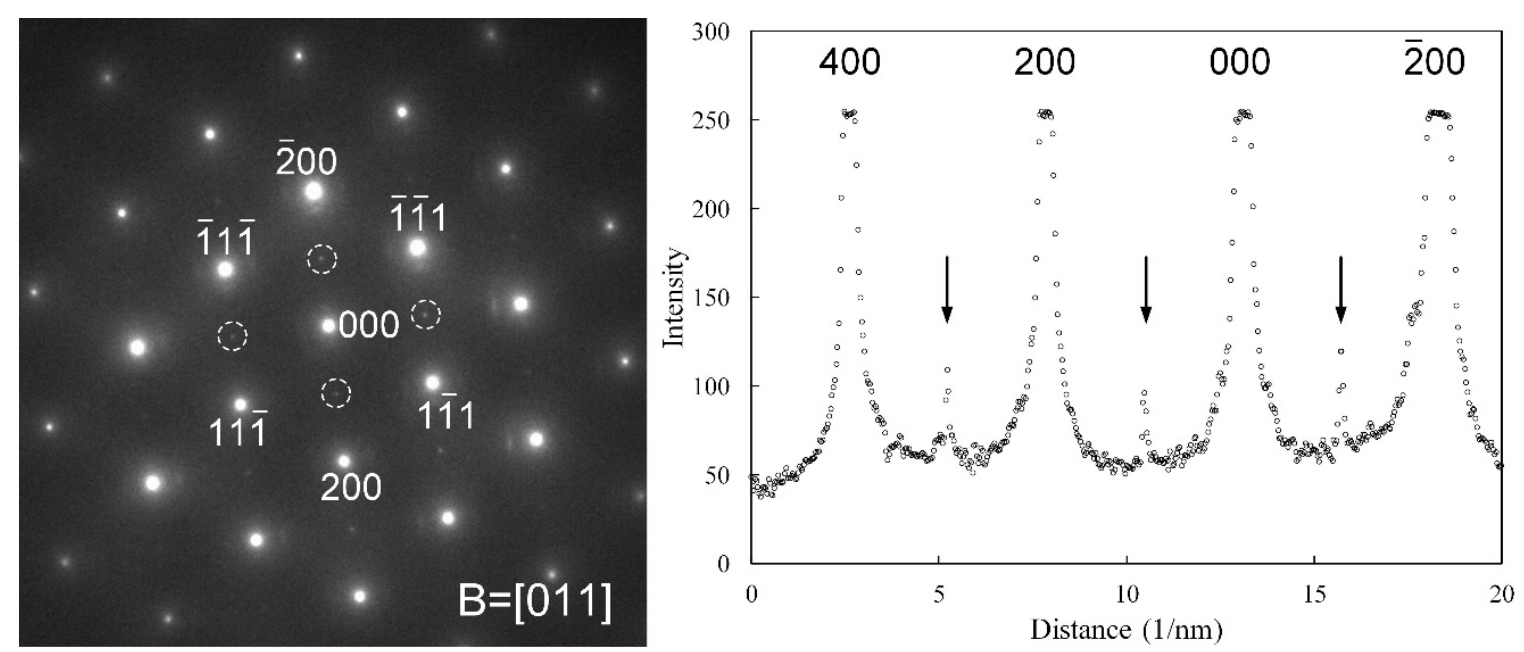

Figure 6-3: TEM diffraction pattern of the matrix containing $\gamma^{\prime}$ precipitates of unirradiated alloy $X-750$, taken along the [011] zone axis. The superlattice reflections from the $\gamma^{\prime}$ precipitates are indicated by dashed circles (left) and arrows (right).
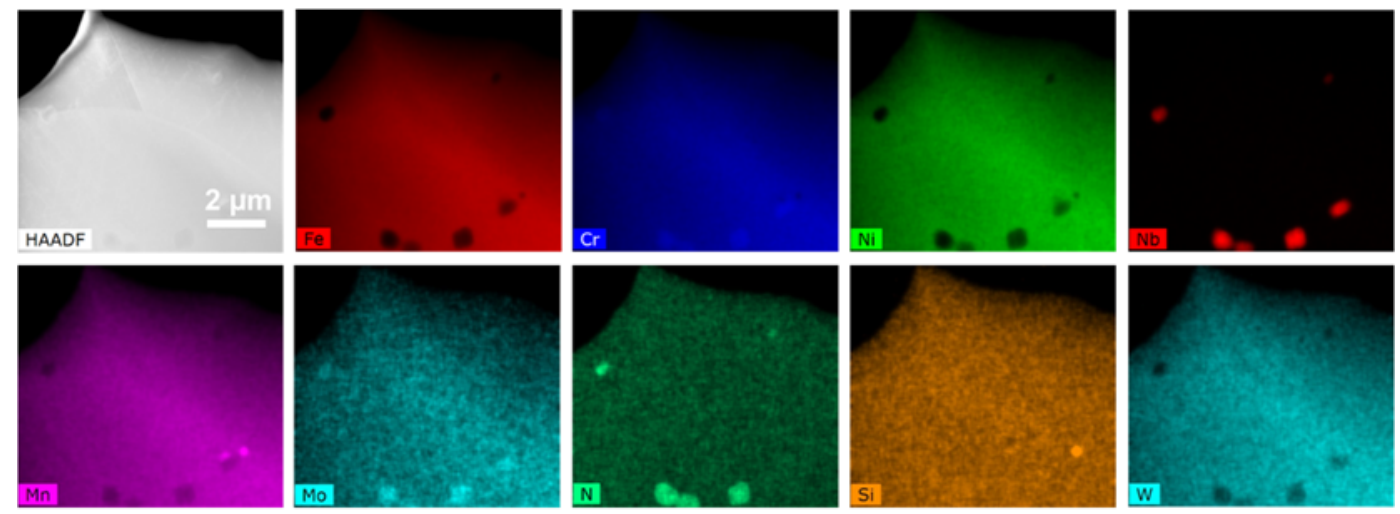

Figure 6-4: STEM-EDS image of unirradiated XM-19 showing coarse (Nb, Mo)-rich nitride precipitates.

\subsection{Evolution of Frank Loops as a Function of Irradiation}

Both alloy X-750 and XM-19 are face-centered cubic structure materials and, at the relatively low irradiation temperatures that these materials were exposed to, the evolution of Frank faulted loops is anticipated in both alloy X-750 and XM-19, leading to embrittlement. Rel-rod dark field (DF) is a wellknown technique for imaging Frank loops. The evolution of Frank loops as a function of dose for alloys X-750 and XM-19 is shown in the rel-rod DF images in Figure 6-5. In alloy X-750, Frank loops were observed at all three irradiation levels, and the loop size increased with increases in fluence. In XM-19, Frank loops were only observed in the medium fluence and high fluence samples, as the defects in the low fluence sample were too small to be resolved and differentiated from FIB damage in the TEM. 

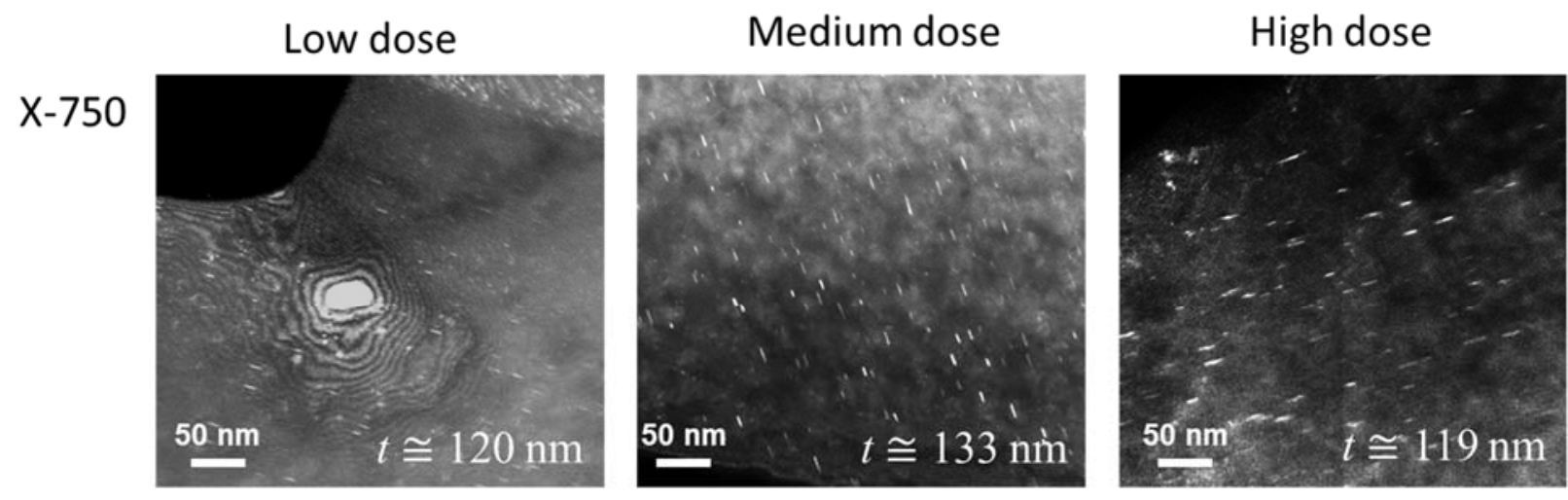

XM-19
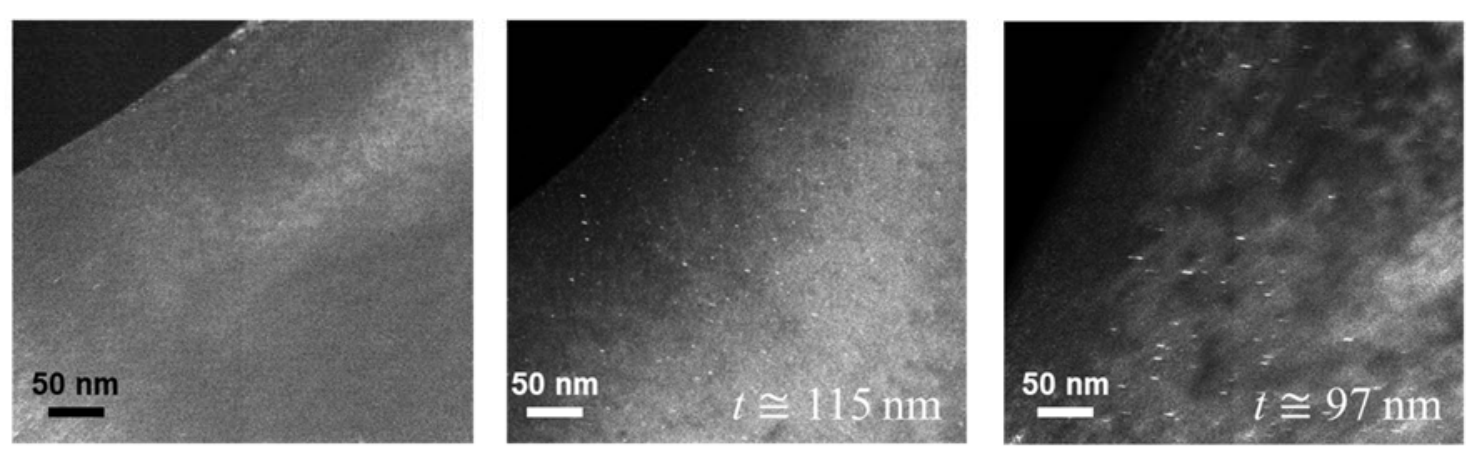

Figure 6-5: TEM rel-rod DF images showing the evolution of Frank loops in alloys X-750 and XM19 irradiated to three different fluences.

Quantitative results of the average size and number density of Frank loops in irradiated alloys X-750 and XM-19 are shown in Figure 6-6 and tabulated in Table 6-1. From low fluence to medium fluence, the Frank loops in alloy X-750 showed a slight increase in size and an apparent increase in number density. From medium fluence to high fluence, the Frank loops in alloy X-750 showed a significant increase in size and a noticeable decrease in number density. In XM-19, the Frank loop size increased from negligible to noticeable in going from low fluence to high fluence, and it became significant from medium fluence to high fluence, with some increase in number density, as well. These observations are consistent with the increasing yield strength and embrittlement in both alloys as fluence increased. It is noted that there are likely other mechanisms at play that contributed to embrittlement - particularly in the case of XM-19, in which yield strength increased significantly - but the general nature of this study did not include more extensive analyses. 

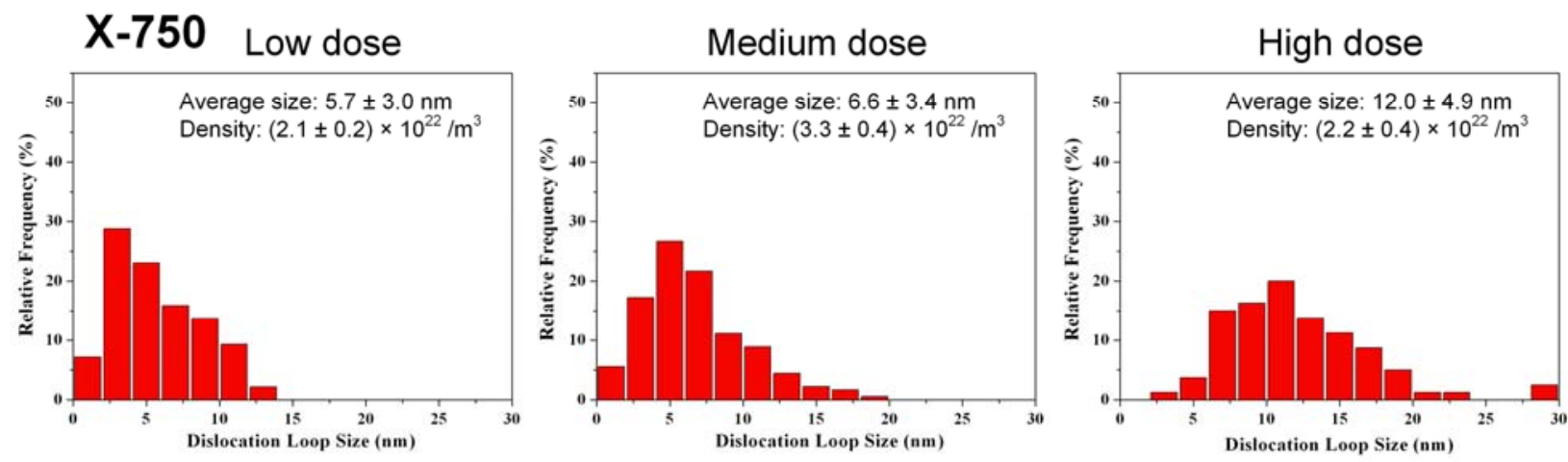

XM-19
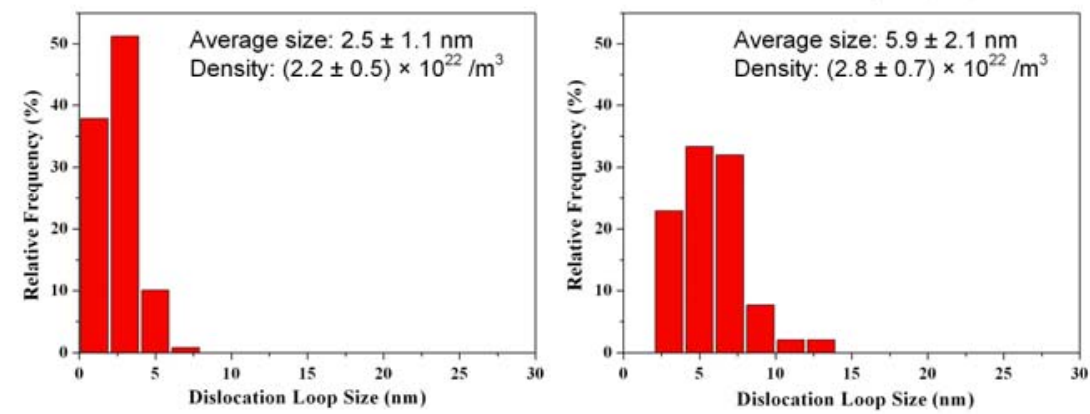

Figure 6-6: Size distributions and number densities of the Frank loops in alloys X-750 and XM-19 irradiated to three different fluences (doses).

Table 6-1: Quantitative statistical data for the average size and number density of the Frank loops irradiated to three different fluences.

\begin{tabular}{|c|c|c|c|}
\hline Sample & $\begin{array}{l}\text { Low dose (EPRI 1) } \\
\left(5.30 \times 10^{19} \mathrm{n} / \mathrm{cm}^{2}\right)\end{array}$ & $\begin{array}{l}\text { Medium dose (EPRI 2) } \\
\left(1.93 \times 10^{20} \mathrm{n} / \mathrm{cm}^{2}\right)\end{array}$ & $\begin{array}{l}\text { High dose (EPRI 3) } \\
\left(9.73 \times 10^{20} \mathrm{n} / \mathrm{cm}^{2}\right)\end{array}$ \\
\hline \multicolumn{4}{|l|}{ Alloy X-750 } \\
\hline Loop size (nm) & $5.7 \pm 3.0$ & $6.6 \pm 3.4$ & $12.0 \pm 4.9$ \\
\hline Loop density $\left(\mathrm{m}^{-3}\right)$ & $(2.1 \pm 0.2) \times 10^{22}$ & $(3.3 \pm 0.4) \times 10^{22}$ & $(2.2 \pm 0.4) \times 10^{22}$ \\
\hline \multicolumn{4}{|l|}{ XM-19 } \\
\hline Loop size (nm) & 0 & $2.5 \pm 1.1$ & $5.9 \pm 2.1$ \\
\hline Loop density $\left(\mathrm{m}^{-3}\right)$ & 0 & $(2.2 \pm 0.5) \times 10^{22}$ & $(2.8 \pm 0.7) \times 10^{22}$ \\
\hline
\end{tabular}

\subsection{Stability of $Y^{\prime}$ Precipitates in Irradiated Alloy X-750}

The stability of $\gamma^{\prime}$ precipitates play an important role in the hardening of alloy X-750 and are known to disorder and dissolve as a function of irradiation damage, thereby resulting in softening of the material [17]. The stability of $\gamma^{\prime}$ is therefore characterized as a function of irradiation dose to ascertain the degree of disordering and dissolution which has taken place. Figure 6-7 is an EDS mapping of the matrix of alloy X-750 irradiated to the highest fluence (EPRI 3). Although the $\gamma^{\prime}$ precipitates cannot be observed in the STEM bright field (BF) image (Figure 6-7 a), the chemical signatures of the $\gamma^{\prime}$ are visible in the Ti and Al EDS maps (Figure 6-7 e and f). This is a strong indication that the precipitates have undergone disordering but no dissolution has occurred, which is consistent with the literature for $\gamma^{\prime}$ stability at these 
low fluences. To determine the degree of disordering, TEM diffraction patterns were captured along the [011] zone axis, as shown in Figure 6-8. Compared to the relative intensity of the $\gamma^{\prime}$ superlattice reflection of unirradiated material (Figure 6-3), it is seen that the superlattice reflections weaken as a function of irradiation (see Figure 6-9). This indicates that, although not fully disordered yet, the $\gamma^{\prime}$ precipitates have begun to disorder.

Combining the STEM-EDS map and the TEM diffraction pattern suggests that, although the $\gamma^{\prime}$ precipitates have not been dissolved, some disordering has occurred as a result of irradiation in alloy X750. The importance of this with respect to mechanical properties is that some amount of hardening from the $\gamma^{\prime}$ precipitates in the form of anti-phase boundaries is removed as a function of irradiation damage; therefore, the material will become softer and more ductile. Of course, this is counteracted by other defects introduced into the material, as discussed in the previous section, and the overall material response has resulted in increased yield strength and reduced ductility.
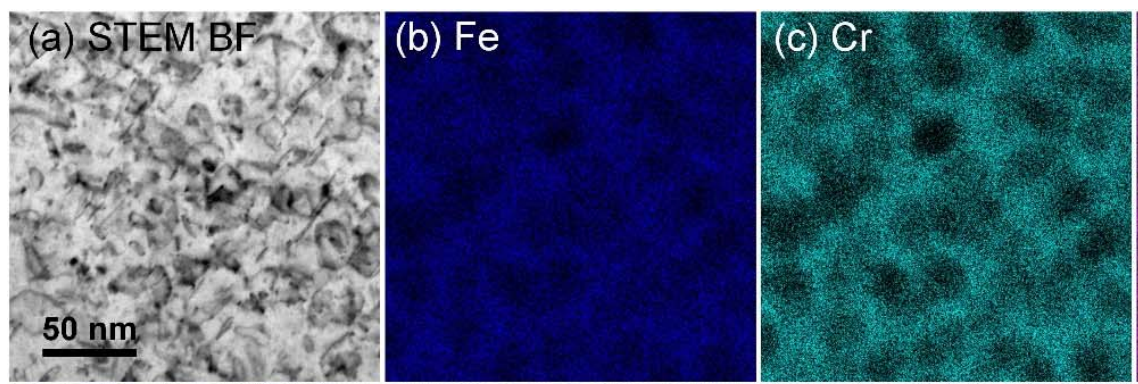

\section{(d) $\mathrm{Ni}$}
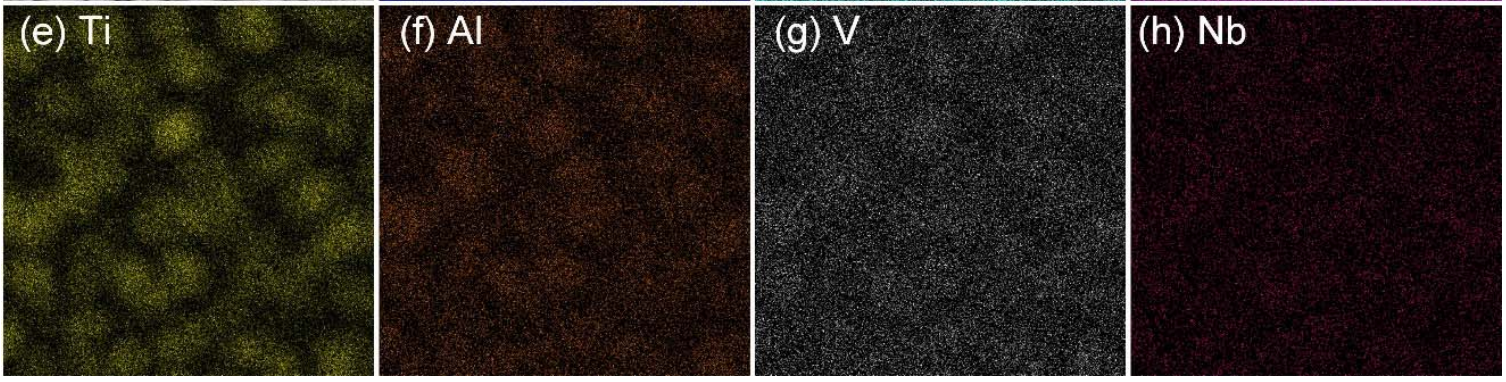

Figure 6-7: STEM-EDS map of the matrix: (a) STEM BF image, (b)-(h) elemental maps of Fe, Cr, $\mathrm{Ni}, \mathrm{Ti}, \mathrm{Al}, \mathrm{V}$, and $\mathrm{Nb}$, respectively. 

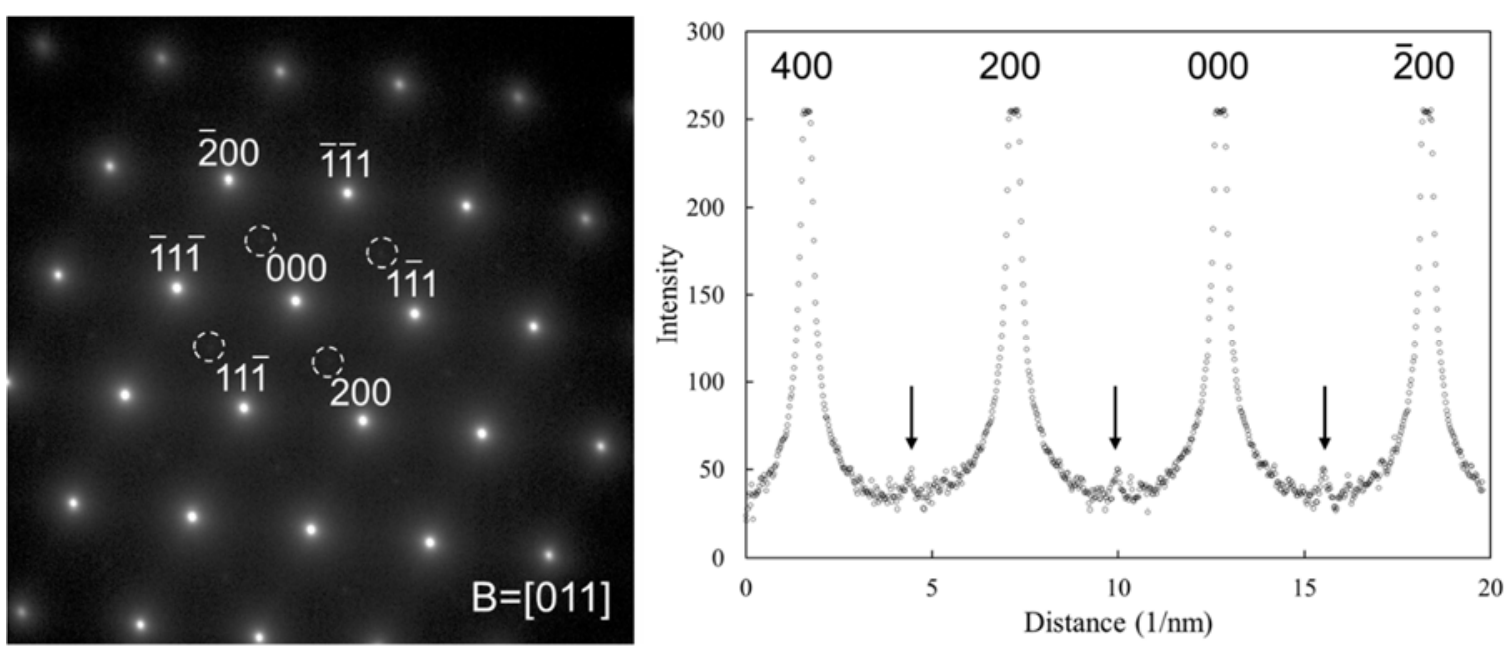

Figure 6-8: TEM diffraction pattern of the matrix containing $\gamma^{\prime}$ precipitates of the high fluence alloy $X-750$, taken along the [011] zone axis. The superlattice reflections from the $\gamma^{\prime}$ precipitates are indicated by dashed circles (left) and arrows (right).

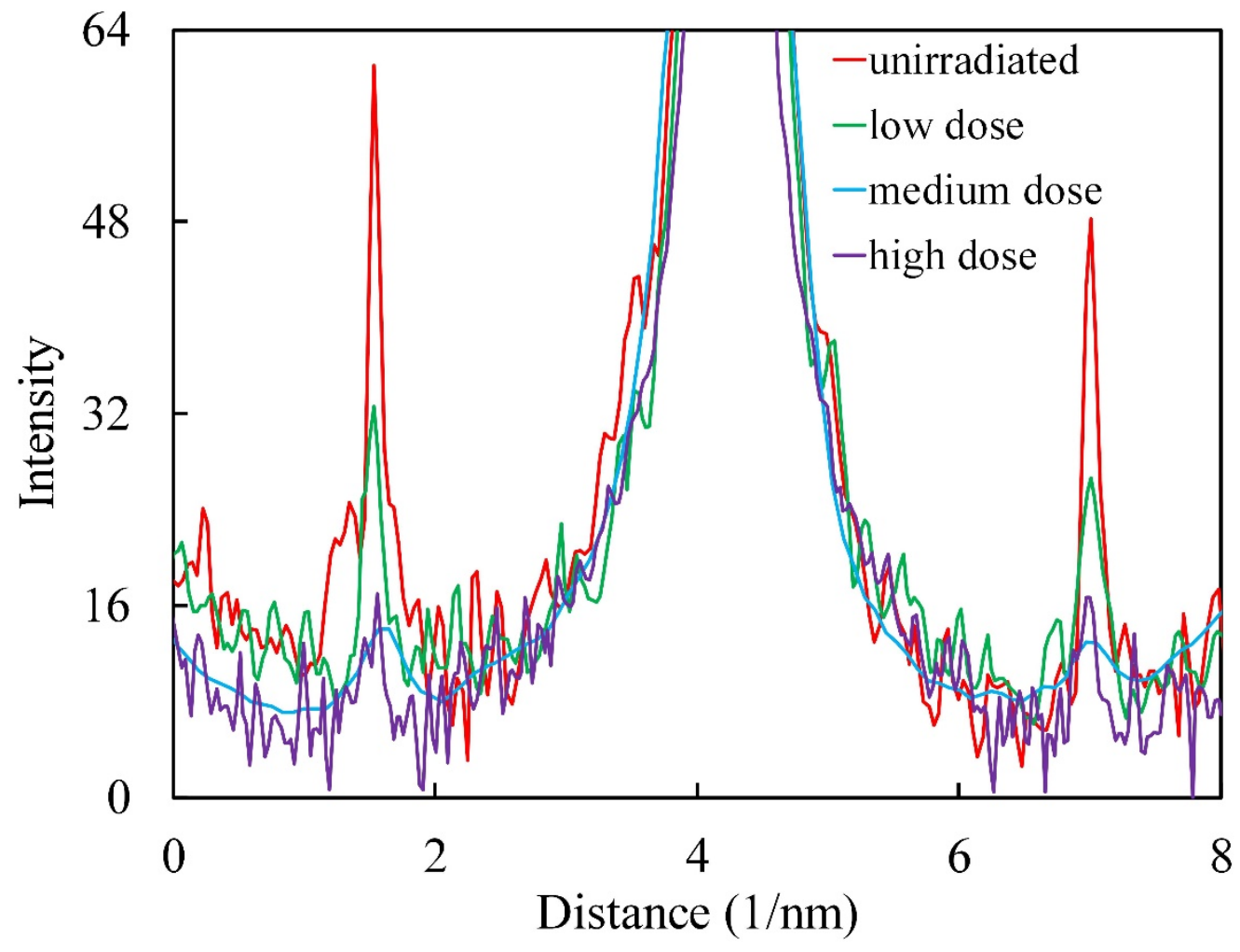

Figure 6-9: Comparison of the relative intensity of the superlattice reflections from the $\gamma^{\prime}$ precipitates in alloy X-750 at different irradiation conditions. The intensity profiles were obtained from diffraction patterns and have been adjusted so that the background signal and widths of the matrix reflection peaks are similar. 


\subsection{Summary of Alloy X-750 and XM-19 TEM Analyses}

The TEM characterization of neutron-irradiated alloys X-750 and XM-19 focused on the evolution of Frank loops in both alloys at different fluences, as well as the stability of ordered $\gamma^{\prime}$ precipitates in X-750. For alloy X-750, Frank loops were observed in all three doses, and the loop size increased with increasing fluence, whereas the loop density increased from low to medium fluence, then decreased from medium to high fluence. For XM-19, few Frank loops were identified at low fluence. From medium to high fluence, the loop size increased, and loop density also increased slightly. $\gamma^{\prime}$ precipitates were found in unirradiated alloy X-750 and all levels of irradiated alloy X-750. The $\gamma^{\prime}$ precipitates showed no evidence of dissolution even at the highest fluence; however, the intensity of the superlattice reflections decreased quickly upon neutron irradiation, suggesting partial disordering of the $\gamma^{\prime}$ precipitates. In general, all of these microstructural observations are consistent with the measured tensile and fracture toughness results.

\section{Discussion and Conclusions}

ASTM standard specimens of alloys X-750 and XM-19 (otherwise known as Nitronic 50) were irradiated in the ATR CFT to three fast fluence targets of $5 \times 10^{19}, 2 \times 10^{20}$, and $1 \times 10^{21} \mathrm{n} / \mathrm{cm}^{2}$ $(\mathrm{E}>1 \mathrm{MeV})$, which correspond roughly to $0.08 \mathrm{dpa}, 0.3 \mathrm{dpa}$, and $1.4 \mathrm{dpa}$ levels of irradiation damage. Since these were the first irradiation experiments to utilize the newly re-installed Loop 2A in the ATR, there were some difficulties encountered with coolant chemistry incompatibility and, as a consequence, imperfect irradiation temperature control. Nonetheless, the specimens are considered useful and representative, and they afforded the opportunity to improve post-irradiation examination processes for this kind of work, as well as to utilize the newly installed IASCC testing capability at INL to provide industry-relevant irradiated fracture toughness and IASCC CGR data, which were scarce or absentparticularly in the case of XM-19. Although they do not provide a strict comparison to studies such as those conducted by Jenssen et al. on alloy X-750 irradiated to approximately $1 \times 10^{19} \mathrm{n} / \mathrm{cm}^{2}[18]$, or by Mills on highly irradiated alloy X-750 [19] [20], they do offer reasonable qualitative values for fracture toughness and IASCC CGR.

Tensile testing showed clear and progressive increases in yield strength and reductions in strain to failure for both alloy X-750 and XM-19. Comparisons to testing performed on unirradiated (baseline) material indicate that, even at the lowest $(\sim 0.08 \mathrm{dpa})$ damage level, some radiation embrittlement is evident. In alloy X-750 tested at $288^{\circ} \mathrm{C}$, the mean $0.2 \%$ offset yield strength increased by approximately 21,35 , and $57 \%$ for low, medium, and high fluences, respectively, compared to baseline mean values. This is consistent with formation of Frank loops in the microstructure that inhibit dislocation movement. Ultimate tensile strength did not increase appreciably, and capacity for strain hardening is reduced as fluence accumulates. For XM-19, the mean $0.2 \%$ offset yield strength measured at $288{ }^{\circ} \mathrm{C}$ exhibited a much higher sensitivity to irradiation embrittlement, with increases of 29,48 , and $89 \%$ for low, medium, and high fluences, respectively, compared to the baseline of $317 \mathrm{MPa}$ reported in [12]. Again, capacity for strain hardening was significantly reduced due to neutron embrittlement in XM-19.

As expected based on the embrittlement noted in the tensile test results, both alloy X-750 and XM-19 exhibited progressive reductions in fracture toughness with the exception of the lowest fluence alloy X750 , which actually showed a slight increase in area under the J-R curve, implying an increased toughness. It is assumed that this is more an artifact of the thinner specimen used for irradiated testing ( $0.4 \mathrm{~T}$ as opposed to $0.5 \mathrm{~T}$ used in baseline testing) than the result of a physical material property evolution, with perhaps some contribution of variations in the microstructure sampled. As noted in [18], there is no significant effect on fracture toughness in alloy X-750, at least at the fluence of $1 \times 10^{19} \mathrm{n} / \mathrm{cm}^{2}$. Only the highest fluence alloy X-750 tests were qualified according to ASTM Standard E1820-09 [4], primarily because specimen sizes were otherwise too small to provide adequate constraint required for plane strain conditions for the low and medium fluence values. This manifested itself primarily in the form of extensive tunneling of cracks (thumbnail-shaped crack front). Although full fracture toughness curves are more appropriate for explicit comparison due to the invalidity of the single point fracture 
toughness values ( $\mathrm{K}$ or J), if the average values of $\mathrm{K}_{\mathrm{JQ}}$ are compared against previously measured values of $\mathrm{K}_{\mathrm{JQ}}$ from unirradiated material, the approximate reduction in fracture toughness of 4,18 , and $41 \%$ for alloy X-750 at the low, medium, and high fluence, as well as 6, 16, and 20\% for XM-19 at the low, medium, and high fluence, does indicate a clear effect. From a rough design engineering perspective, the remaining fracture toughness in both alloys remains quite high at an irradiation damage level of $\sim 1.4 \mathrm{dpa}$ (representative of a $60-80$ year BWR life), though, clearly, alloy X-750 has transitioned from elasticplastic to linear elastic, which is more prone to rapid fracture events.

A large volume of the IASCC CGR data obtained in this project is comparable to tests conducted previously on unirradiated specimens of both alloys. Effects of switching between high corrosion potential (NWC) and low corrosion potential (HWC) environments were measured, and it was confirmed that there is a significant reduction in CGR in HWC environments. Tests conducted at a range of different applied stress intensity factors $(\mathrm{K})$ also showed a typical $\mathrm{K}^{2}$ dependence for CGR. For both alloys, the measured CGRs were on par with those measured in unirradiated materials of the same heat, suggesting that IASCC CGR is less sensitive to irradiation embrittlement in these alloys than are tensile or fracture toughness properties. At high corrosion potential, there is only limited evidence that the higher fluence specimens ( $\sim 1.5 \mathrm{dpa})$ exhibit a higher SCC growth rate than do the lower fluence specimens ( $\sim 0.3 \mathrm{dpa})$. The effect at low corrosion potential (HWC) indicates a larger effect of fluence, with the $\sim 1.5$ dpa data in almost all cases showing higher CGR than the $\sim 0.3$ dpa data.

TEM analyses support the increase in yield strength observed in tensile tests of both alloys, though the limited analyses conducted in this project focused almost exclusively on the evolution of Frank loops. The substantial increase in yield strength of XM-19 relative to baseline values imply that there are additional mechanisms at work that likely could be investigated. Onset of the disordering of $\gamma^{\prime}$ precipitates in alloy X-750 was noted, although dissolution of these precipitates has not occurred, implying that stabilization of material property evolution likely occurs at a higher fluence.

Although there clearly are opportunities for additional research on the remaining materials from this project (primarily in regard to microstructural analysis), the project is considered a success overall. It is shown that both materials retain a high level of integrity even at the highest fluence, synonymous with beyond end-of-life for BWR components that utilize these materials. Valuable experience was gained in testing protocol and processes, and the path was paved for future NSUF experiments on structural materials involving IASCC.

\section{References}

[1] J. H. Jackson and S. P. Teysseyre, "Baseline Fracture Toughness and CGR Testing of Alloys X-750 and XM-19 (Phase I)," Idaho National Laboratory, Idaho Falls, 2012.

[2] C. Tyler and J. Neilsen, "EPRI Phase II report," Idaho National Laboratory, Idaho Falls, 2012.

[3] P. L. Andresen, J. Flores-Preciado, M. M. Morra and R. G. Carter, "Microstructure and SCC of Alloy X-750," in Proceedings of the 15th International Conference on Environmental Degradation of Materials in Nuclear Power Systems-Water Reactors, 2011.

[4] ASTM E-1820-09: Standard test method for measurement of fracture toughness, West Conshohocken, PA: ASTM International, 2009.

[5] ASTM E8-04: Standard Test Methods for Tension Testing of Metallic Materials, West Conshohocken, PA: ASTM International, 2004.

[6] P. E. Murray and J. H. Jackson, "As-run thermal hydraulics analysis of the EPRI-1 Experiment," Idaho National Laboratory, Idaho Falls, 2020. 
[7] J. R. Mitchell and J. H. Jackson, "As-run physics analysis for the EPRI-1 experiment," Idaho National Laboratory, Idaho Falls, 2020.

[8] P. E. Murray and J. H. Jackson, "As-run thermal hydraulics analysis of the EPRI-2 experiment," Idaho National Laboratory, Idaho Falls, 2020.

[9] J. W. Nielsen and J. H. Jackson, "As-run physics analysis for the EPRI-2 experiment for cycle 153B," Idaho National Laboratory, Idaho Falls, 2020.

[10] P. E. Murray and J. H. Jackson, "As-run thermal-hydraulics analysis of the EPRI-3 experiment," Idaho National Laboratory, Idaho Falls, 2020.

[11] C. M. Hill and J. H. Jackson, "As-run physics analysis for the EPRI-3 experiment for cycles 155B and 158B," Idaho National Laboratory, Idaho Falls, 2020.

[12] ATI Allegheny Ludlum, "ATI 50 / ATI XM-19 Stainless Steel," 13 April 2020. [Online]. Available: https://www.atimetals.com/Products/ati-50-ati-xm-19. [Accessed 13 April 2020].

[13] P. Andresen, M. Morra and R. Carter, "SCC and Fracture Toughness of XM-19," in Proceedings of the 18th International Conference on Environmental Degradation of Materials in Nuclear Power Systems - Water Reactors, Portland, Oregon, 2017.

[14] P. L. Andresen and R. G. Carter, "Microstructure, SCC and J-R Fracture Resistance of Alloy X-750 and XM-19," Electric Power Research Institute, Palo Alto, 2017.

[15] P. Andresen and R. G. Carter, "Development and Analysis of an Alloy X-750 SCC Growth Rate Database," in Proceedings of the 17th International Conference on Environmental Degradation of Materials in Nuclear Power Systems - Water Reactors, 2015.

[16] P. Andresen and M. M. Morra, "Microstructure, SCC and J-R Fracture Resistance of Alloy X-750 and XM-19," Electric Power Research Institute, Palo Alto, 2015.

[17] C. D. Judge, H. Rajakumar, A. Korinek, G. Botton, J. Cole, J. W. Madden, J. H. Jackson, P. D. Freyer, L. A. Giannuzzi and M. Griffiths, "High Resolution Transmission Electron Microscopy of Irradiation Damage in Inconel X-750," in Proceedings of the 18th International Conference on Environmental Degradation of Materials in Nuclear Power Systems - Water Reactors, Portland, 2017.

[18] A. Jenssen, P. Efsing and J. Sundberg, "Influence of heat treatment, aging and neutron irradiation on the fracture toughness and crack growth rate in BWR environments of alloy X-750," in Proceedings of the 12th International Conference on Environmental Degaradation of Materials in Nuclear Power Systems - Water Reactors, Salt Lake City, 2006.

[19] W. J. Mills, "Effect of Temperature on the Fracture Toughness Behavior of Inconel X-750," in Fractography and Materials Science, ASTM STP 733, L. N. Gilbertson and R. D. Zipp, Eds., American Society for Testing and Materials, 1981, pp. 98-114.

[20] W. J. Mills, "Postirradiation fracture toughness of Inconel X-750," Engineering Fracture Mechanics, vol. 18, no. 3, pp. 601-607, 1983.

[21] T. L. Anderson, Fracture Mechanics: Fundamentals and Applications, CRC Press, Inc., 1995.

[22] "BWRVIP-240: BWR Vessels and Internals Project, Metallurgical Analyses and Macro and Microstructural Mapping of Alloy X-750 and Alloy XM-19 Plates," EPRI, Palo Alto, CA, 2010. 